\title{
Vaginal penetration: pain or pleasure?
}

Citation for published version (APA):

Melles, R. J. (2019). Vaginal penetration: pain or pleasure? The role of fear and sexual arousal. [Doctoral Thesis, Maastricht University]. ProefschriftMaken. https://doi.org/10.26481/dis.20190920rm

Document status and date:

Published: 01/01/2019

DOI:

10.26481/dis.20190920rm

Document Version:

Publisher's PDF, also known as Version of record

\section{Please check the document version of this publication:}

- A submitted manuscript is the version of the article upon submission and before peer-review. There can be important differences between the submitted version and the official published version of record.

People interested in the research are advised to contact the author for the final version of the publication, or visit the DOI to the publisher's website.

- The final author version and the galley proof are versions of the publication after peer review.

- The final published version features the final layout of the paper including the volume, issue and page numbers.

Link to publication

\footnotetext{
General rights rights.

- You may freely distribute the URL identifying the publication in the public portal. please follow below link for the End User Agreement:

www.umlib.nl/taverne-license

Take down policy

If you believe that this document breaches copyright please contact us at:

repository@maastrichtuniversity.nl

providing details and we will investigate your claim.
}

Copyright and moral rights for the publications made accessible in the public portal are retained by the authors and/or other copyright owners and it is a condition of accessing publications that users recognise and abide by the legal requirements associated with these

- Users may download and print one copy of any publication from the public portal for the purpose of private study or research.

- You may not further distribute the material or use it for any profit-making activity or commercial gain

If the publication is distributed under the terms of Article $25 \mathrm{fa}$ of the Dutch Copyright Act, indicated by the "Taverne" license above, 
(C) R.J. Melles, Bemelen 2019

$\begin{array}{ll}\text { Cover image } & \text { Michel Geelen } \\ \text { Cover design } & \text { Michel Geelen } \\ \text { Lay out } & \text { Reinhilde Melles, Michel Geelen } \\ \text { Print } & \text { ProefschriftMaken Maastricht } \\ \text { ISBN } & 9789463804646\end{array}$




\section{Vaginal penetration: pain or pleasure?}

\section{The role of fear and sexual arousal}

Proefschrift

Ter verkrijging van de graad van doctor aan de Universiteit Maastricht, op gezag van de Rector Magnificus, Prof.dr. Rianne M. Letschert

volgens het besluit van het College van Decanen,

in het openbaar te verdedigen

op vrijdag 20 september 2019 om 14.00 uur

door

\section{Reinhilde Johanna Melles}

Geboren op 4 november 1964 te Alblasserdam 


\section{Promotor}

Professor dr. M.L. Peters

\section{Copromotores}

Dr. M.M. ter Kuile, Leids UMC

Dr. M.V.E. Dewitte

\section{Beoordelingscommissie}

Prof. dr. A.A.N. Mulkens (Chair)

Prof. dr. S. Bergeron, Université de Montreal, Canada

Dr. J.E. Hartog, Dept. of Gynecology

Prof. dr. E.T.M. Laan, AMC Amsterdam

Prof. dr. R.W.H.M. Ponds

The research described in this thesis was performed at the School of Experimental Psychopathology, section Experimental Health Psychology, Faculty of Psychology and Neuroscience, Maastricht University. 


\section{CONTENT}

$\begin{array}{lll}\text { Chapter } 1 \quad \text { Introduction } & 07\end{array}$

Chapter 2 Attentional bias for pain and sex, and automatic 23

appraisals of sexual penetration: Differential patterns in dyspareunia versus vaginismus?

Chapter 3 Automatic and subjective affective associations with 41 sexual stimuli in women with lifelong vaginismus before and after therapist-aided exposure treatment.

Chapter $4 \quad$ Therapist-Aided Exposure for Women with Lifelong 69 Vaginismus: Mediators of Treatment Outcome: A Randomized Waiting List Control Trial

Chapter 5 The Vaginal Pressure Inducer: a new device to test the 93 (un)pleasurableness and tolerance of vaginal pressure and the influence of sexual stimuli.

Chapter 6 Vaginal Pressure: Pain or Pleasure? The role of 111 individual differences and context-dependent expectations in sexual and non-sexual contexts.

Chapter $7 \quad$ Summary and general discussion

Knowledge valorization

Samenvatting

References

Dankwoord

Curriculum Vitae

Publications 

CHAPTER

\section{Introduction}


Roos, 28 years: "Intercourse really hurts; it feels like a knife is cutting me. The pain after intercourse remains for hours and even having a pee then burns and stings. In the early days of our relationship we really enjoyed sex, but around five years ago we had a very busy and quite stressful time. That's when the pain began. I think I wasn't in the mood for sex. But I didn't want to let him know that it hurt, so I bit the bullet. But over time, it's become worse. Now I don't like him to touch me there, because it feels so... dry and I'm worried he wants more. Sometimes, I let him do what he wants and I hope it will be over as soon as possible. But it's becoming more and more difficult to let him inside me, it feels tight. The physician couldn't find anything wrong with me. She advised lubricants, but that doesn't seem to help. This has been going on for years now. And, now, when he tries to hug me, I think: "O no, there we go..." and then I push him away, don't want to turn him on. My boyfriend is very patient, but he's a man you know.... And sex is important... I 'm afraid he can't bear this much longer..."

Aisha, 32 years: "I'm not able to have sex [she means sexual penetration], never been. It feels locked; I thought I didn't have an opening there. I've never tried to insert anything there. When we were married, we tried to have intercourse, but it was totally blocked. We've tried it several times, but because we didn't succeed we stopped trying. Mahmed has really helped me to enjoy intimacy. I've had some negative experiences, you know... But he was very patient, and now, I really like him to touch me. I can get excited. Yes, and I can even have an orgasm!. Now we want to get pregnant, so we went to a physician. I was very worried about that. I was so afraid that it would be painful... and there would be something wrong with me. But the physician couldn't do the exam. She said when she touched me there, I cramped completely. She reassured me that she'd noted no pathology. I first have to learn how to relax, so she can examine me inside. That's why she has referred me. And yes, now we're here, we would like to be able to have sexual penetration as well. It would complete our relationship."

\section{GENITAL PAIN DISORDERS}

The difficulties in having intercourse reported by Roos and Aisha are not exceptional. Chronic genital pain is common among women; population-based estimates report prevalence rates ranging from 5 to $9 \%{ }^{3-6}$, with higher prevalence rates (13\%) among 
young women (20-29 yrs) ${ }^{7}$. Terms used in the literature to describe genital pain include intercourse pain, sexual pain, focal vulvitis, vulvar vestibulitus syndrome, provoked/unprovoked vulvodynia/vestibulodynia and dyspareunia ${ }^{8}$.

Genital pain, fear of genital pain, and tensing or tightening of the pelvic floor muscles during attempted vaginal penetration are core symptoms of the DSM 5 defined Genitopelvic pain/penetration disorder (GPPPD) ${ }^{9}$. The category of GPPPD has resulted from the merging of two distinct classifications defined in the earlier DSM IV TR: dyspareunia and vaginismus.

In the case of dyspareunia, women are able to have intercourse, but experience recurrent genital pain associated with sexual intercourse that causes distress and interpersonal problems 10,11 .

The genital pain is most commonly experienced in the vulva and/or vaginal entrance, socalled superficial dyspareunia, and is usually provoked by tactile stimulation by the penis, the use of a tampon or other forms of friction of the vulvar or vaginal skin. Deep dyspareunia is perceived to be located in the abdominal or internal organs, often associated with penile thrusting ${ }^{12}$. In deep dyspareunia, the pain is usually related to a physical condition, and this type of pain occurs in a minority of women with genital pain. It is therefore beyond the scope of this thesis. Dyspareunia is referred to as lifelong if it has been present throughout life, whereas secondary dyspareunia is acquired and develops after a non-symptomatic period. The repeated experience of pain during intercourse may result in women tightening their pelvic floor muscles, which further impedes vaginal penetration 13,14. Consequently, some of these women develop secondary vaginismus over time, which can be described as "persistent difficulties to allow vaginal entry of a penis, a finger, and/or any object, despite the woman's expressed wish to do so" 15 .

In contrast to secondary vaginismus, lifelong vaginismus implies that a woman has never been able to have intercourse. The prevalence of vaginismus is not well established, although it is estimated to range between $1 \%$ and $6 \% 16$. Many women with vaginismus also experience pain upon touch (40-100\%) ${ }^{17,18}$. 
To carefully differentiate between the two genital pain disorders, the current thesis focuses on women with lifelong vaginismus and superficial dyspareunia. In accordance with the DSM V, this thesis uses the term "genital pain" to refer to both dyspareunia and vaginismus.

\section{Treatment-seeking behaviour}

Unfortunately, a recent review found that only $60 \%$ of women with genital pain reported to have sought treatment; $52 \%$ of these women had never received a formal diagnosis ${ }^{19}$. In the Netherlands, only $37 \%$ of women who had a sexual problem (e.g. genital pain) and felt the need to seek help actually contacted a health professional, and only $6 \%$ of this group obtained advice or help ${ }^{3}$. A number of barriers to seeking help have been reported by women with genital pain, and these barriers resulted in delayed diagnosis and treatment, with deleterious consequences for sexual functioning, wellbeing, and relationships 19. Reasons for not seeking professional advice included embarrassment and lack of confidence in a medical solution 20 .

When confronted with women suffering from genital pain, many health professionals and pain experts feel uncomfortable about dealing with pain located in the genital area ${ }^{8}$. They also feel uncertain about treatment options, and as a result do not engage in a proactive approach 21 . The nosological confusion does not help either when it comes to developing expertise in genital pain disorders ${ }^{8}$. The current thesis aims to offer deeper insight into the mechanism of genital pain and ways to remedy this.

\section{Multifactorial aetiology}

Traditionally, research on genital pain has focused on studying biomedical factors, thereby disregarding the sexual and emotional impairments associated with genital pain ${ }^{8}$. In recent years, the psychosexual field has taken a huge leap forward, yielding important insights into the psychosocial factors that are particularly important for an understanding of the persistence and exacerbation of genital pain. It is now commonly 
accepted that the biopsychosocial model has the greatest heuristic value for assessing, understanding, and treating genital pain ${ }^{22}$. A brief overview of the biological and psychosocial correlates of genital pain is presented below. Subsequently, an introduction is provided to commonly used theoretical models to understand genital pain and sexual responding, together with supporting evidence. Finally, this chapter presents missing or conflicting evidence regarding the models, followed by an outline of the current thesis.

\section{Biological factors}

Studies on the physical and physiological factors related to genital pain have focused mainly on causality. Even though there is conflicting evidence about biological causes of chronic (unexplained) genital pain, several pathways to modify the risk of developing genital pain have been suggested (see for an overview of studies: ${ }^{8,19}$ ). The most consistent evidence regards the role of hypertonic pelvic floor muscles. The increased activity of the pelvic floor muscles may be associated with some degree of pelvic floor muscle dysfunction ${ }^{19}$, as well as a response to fear ${ }^{23,24}$. Since hormonal contraception seems to predict genital pain, it has been suggested that hormonal changes may induce morphologic changes in the vestibular mucosa, which may increase its sensitivity. Furthermore, female genital pain has been found to be associated with a general/central hypersensitivity. This may be explained by neurological changes in the vulvar nerve fibre density in vulvodynia. In addition, inflammation due to repeated vulvovaginal (Candida) infections has been suggested as a potential cause, as this may lead to increased vulvar innervation. For all suggested biological factors involved in genital pain, it remains unclear whether these are causes or consequences of genital pain. Most likely, bidirectional relationships exist.

As regards lifelong vaginismus, a physical explanation, for example congenital hymeneal or vaginal abnormalities, is rarely found $(0-5 \%)$ 13,17,25. 


\section{Psychosocial factors}

Research on psychosocial variables has focused mainly on understanding how pain is maintained and exacerbated. Since the 1970s, the behavioural perspective on genital pain has dominated research and clinical practice. Genital pain, especially vaginismus, has been described as a learned conditioned reflex, explained by a negative or traumatic experience with vaginal penetration caused by rape, a clumsy use of tampon, urological or gynaecological examination or a clumsy first lover $26,27$.

Researchers have also studied psychological profiles by comparing women with and without genital pain, and found a wide range of psychological factors associated with genital pain. Women with genital pain reported higher levels of neuroticism, pessimism, trait anxiety, perfectionism, harm avoidance, disgust propensity (mainly in the case of vaginismus, and to a certain extent in dyspareunia), and fear of negative evaluations, as well as reduced levels of self-esteem, optimism, extraversion and self-efficacy ${ }^{8,19}$. A higher degree of erotophobia and reduced sexual pleasure have also been reported, as well as greater difficulties with sexual arousal and lubrication during partnered sexual activities ${ }^{8}$. Since most of these studies have been cross-sectional, it remains unclear whether these factors preceded pain or developed after the pain began. Traumatic experiences seem to explain only a small part of the prevalence of genital pain ${ }^{22,28}$.

Social factors have long been neglected in the study of genital pain, which is particularly surprising as the pain often occurs in a relational context. In the last decade, research into social factors has made substantial progress. Interpersonal dynamics like open communication about sexual matters, relationship satisfaction and hostile, solicitous and facilitative partner responses to pain may play a role in regulating emotions and pain behaviour 29-31. These interpersonal dynamics may interact with intrapersonal factors and the social context, including attachment orientation and the social genderrelated norms towards sexuality ${ }^{31,32}$ (see for an overview: ${ }^{8}$ ). Although this interactional approach to the study of genital pain seems promising, the current thesis mainly focuses on psychological mechanisms of genital pain. 


\section{Circular Cognitive Behavioural model}

To better understand how genital pain is maintained and exacerbated, Spano and Lamont (1975) introduced the first cognitive behavioural model of genital pain, describing how women get trapped into a vicious circle of genital pain ${ }^{33}$. Together with the fear-avoidance model by Vlaeyen and Linton ${ }^{34}$, this model was the starting point for the development of the Circular Cognitive Behavioural Model of genital pain, (see Figure 1: CB model) ${ }^{2}$. This model assumes that memories of pain or negative experiences with penetration can lead to negative penetration beliefs (e.g. catastrophic pain/penetration cognitions) as well as fear of pain in new intercourse situations. The fear (of pain) is associated with impaired sexual arousal, along with increased hypervigilance, decreased genital arousal (lubrication) and increased pelvic floor muscle tension. When penetration is attempted, the combination of vaginal dryness and increased pelvic floor muscle tension result in greater friction between the penis and the vulvar skin. This mechanical friction may result in pain, even tissue damage and/or the impossibility of vaginal penetration (vaginismus) by which the vicious pain-penetration circle is completed. To cope with the fear, some women avoid all activities involving vaginal penetration..

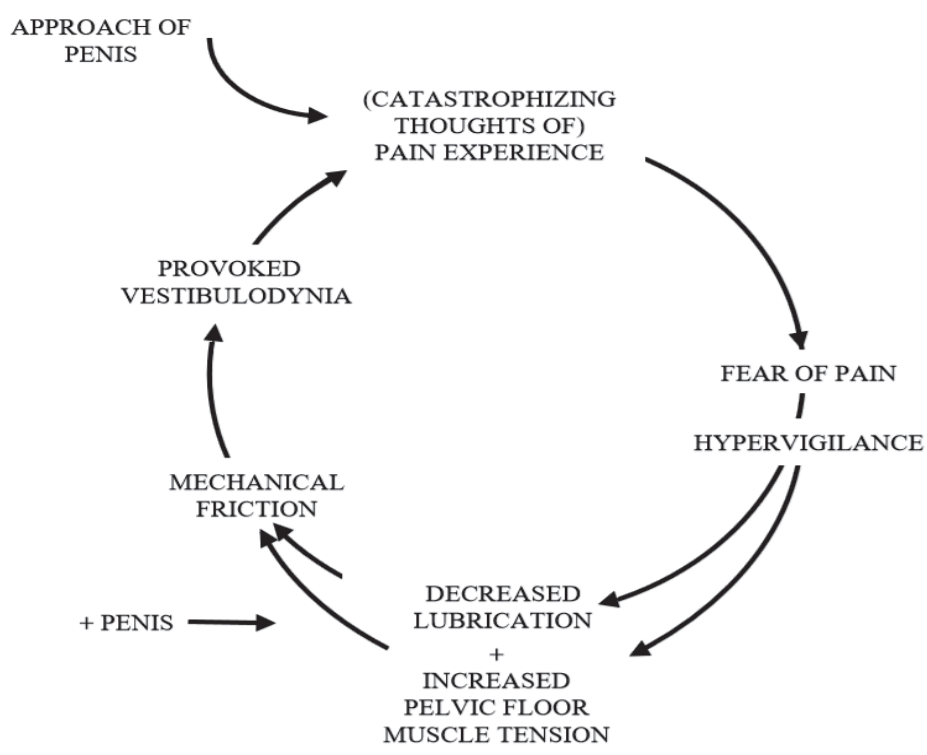

Figure 1: Circular cognitive behavioural model of dyspareunia , ter Kuile et al ${ }^{2}$. 
Elements of this CB model have received some empirical support. Studies in women with genital pain have reported higher levels of pain catastrophizing and more negative and less positive penetration cognitions compared with controls $35-38$. Women with genital pain also reported relatively negative affective associations with sexual penetration stimuli (e.g, increased fear and aversion ${ }^{39-41}$ ). Furthermore, bi-directional relationships have been found between repeated genital pain experiences and increased fear of pain, hypervigilance for pain cues and pain catastrophizing cognitions, leading to further impairment of sexual arousa $\mathbf{1}^{36,42-45}$. Women with genital pain reported lower levels of sexual arousal in response to various sexual stimuli than sexually functional women 46,47. In addition, women with genital pain showed higher levels of pelvic floor muscle tension during a gynaecological examination than sexually functional women $13,18,48$. Based on the CB model for genital pain avoidance behaviour towards vaginal penetration can be expected. Accordingly, women with vaginismus showed relatively strong fear-avoidance tendencies to vaginal penetration or attempts of penetration (see Figure. 2) 2,18,49. Also, a proportion of the dyspareunia group showed decreasing motivation to engage in sex over time 50,51. This avoidance behaviour has been found to mediate the relation between pain catastrophizing and genital pain ${ }^{51}$. However, the majority of women with dyspareunia (68\%) indicated that they continued to have intercourse despite the pain ${ }^{52}$. This persistence behaviour in painful intercourse cannot be explained by the CB model of genital pain ${ }^{8}$.

Both avoidance and persistence behaviour may increase pain catastrophizing cognitions, fear of pain and hypervigilance. Avoidance behaviour towards vaginal penetration stimuli prevents desensitization, while persisting in painful penetration confirms genital pain sensations. Both may increase genital pain sensitivity, along with increased pelvic floor muscle tension, resulting in impaired sexual arousal 52-54. This may impair the opportunity to appraise genital sensations as a pleasant experience. 


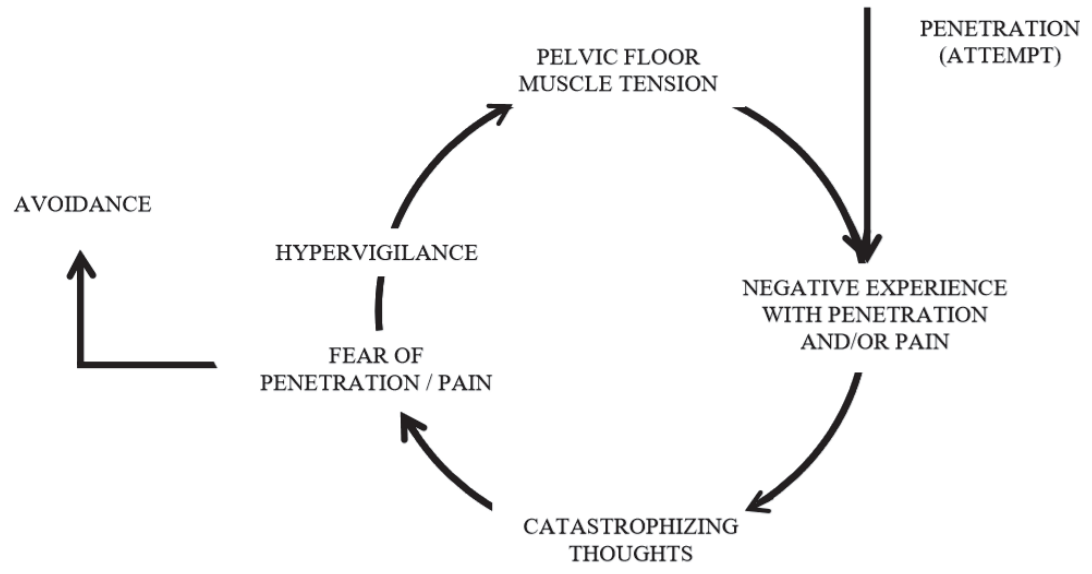

Figure 2: Circular fear-avoidance model of vaginismus, ter Kuile et al. ${ }^{2}$.

Drawing on the CB models, current treatments of genital pain have focused mainly on reducing fear of pain, hypervigilance/pain sensitivity and pelvic floor muscle contraction. Furthermore, interventions aim to modify catastrophic pain cognitions and to increase the pleasurable sexual repertoire, for example by prohibiting pain-provoking behaviour ${ }^{2}$. CBT has proven to be effective for women with dyspareunia, although the observed effect sizes were modest, and only 40-50\% of the participants were engaging in intercourse after the treatment ${ }^{55,56}$. The protocol was less effective for women with vaginismus, due to their strong avoidance behaviour towards vaginal penetration stimuli ${ }^{57}$. Consequently, a new approach was developed using interventions specifically focusing on conquering fear and avoidance by gradual exposure in vivo, accompanied by a therapist. Therapist-aided exposure treatment has proven to be highly effective. After treatment, $89 \%$ of women were able to have intercourse 58,59 , and fear and negative penetration cognitions were successfully reduced ${ }^{58}$.

Several research findings have contradicted the CB model of genital pain. According to the model, fear of pain reduces genital arousal (lubrication) and increases pelvic floor muscle activity. However, these physical responses are observed in both women with and without genital pain 23,24,36,44,60,61. Moreover, the increased activity of the pelvic floor muscles is not exclusively associated with fear; positive emotions such as high levels of 
(genital and subjective) sexual arousal have also been found to elicit activity of the pelvic floor muscles 61-65. Furthermore, studies found contradicting results on the suggested impaired genital arousal in women with genital pain: two studies found lower genital responsiveness in women with genital pain than nonaffected women, 47,66; other studies found no significant differences between women with and without genital pain in terms of their genital arousal response to sexual stimuli $46,67,68$. Using masturbation as a sexual stimulation, studies failed to find significant differences in sexual arousal, lubrication and pain between women with and without genital pain ${ }^{69}$. This suggests that affected women seem to be capable of a normal sexual response when the context does not involve a threat of pain or aversion ${ }^{8}$.

\section{Information Processing Model of Sexual Arousal}

The discrepancy between an intact physical sexual arousal and an impaired subjective sexual arousal in response to visual sexual penetration stimuli in women with genital pain suggests that sexual (penetration) stimuli may have acquired ambivalent meanings, depending on the context. The notion that sexual stimuli or situations may have multiple meanings and thus that sexual arousal may co-occur with negative emotional responses to sexual stimuli or emotional states (like anxiety) is explained by the Information Processing Model of Sexual Arousal developed by Janssen and colleagues (see Figure. $3)^{1}$.

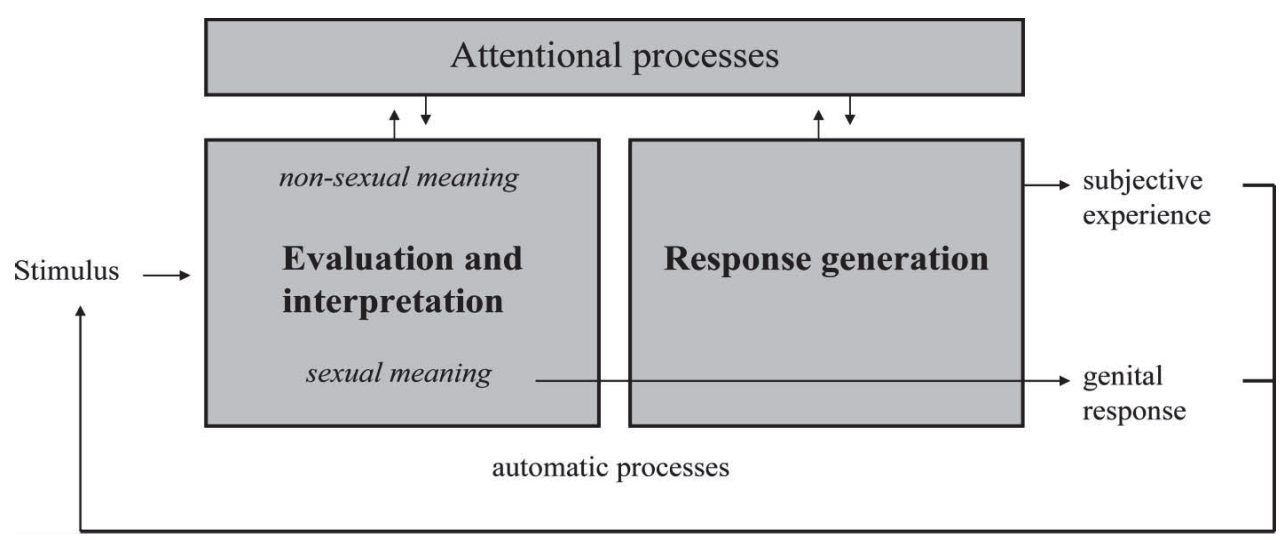

Figure 3: Information Processing Model of Sexual Arousal Janssen et al. 1 
This model has clear heuristic value when it comes to understanding how sexual stimuli are processed and how this might be influenced by (multiple as well as ambivalent) automatic and subjective associations. According to the model, there are two information processing paths of sexual stimuli: an automatic path (i.e., reflexive, spontaneous, efficient) triggering genital responses, and a conscious path (i.e., reflective, controlled, effortful), leading to subjective sexual arousal ${ }^{1}$. It is assumed that sexual stimuli automatically attract the attention and trigger an automatic appraisal of the sexual meaning of the stimuli, which then automatically elicits genital arousal. In a subsequent step, the awareness of becoming sexually aroused becomes part of the stimulus event, resulting in a conscious (i.e. explicit) appraisal of the sexual stimuli (like genital sensations), which maintains attention towards the sexual stimulus ${ }^{1}$. Applied to genital pain, the model assumes that when sexual penetration is painful or impossible, a sexual (penetration) stimulus is automatically associated with pain or fear of pain, and attention is preferentially directed towards threatening (painful) aspects of having sex. This results in decreased attention for sexual stimuli, along with more negative explicit appraisals of sexual stimuli, reduced explicit and genital sexual arousal and higher pelvic floor tension ${ }^{1,43,67,70,71}$.

Although the information processing model is helpful to understand the cognitive processes that may be involved in genital pain, the role ascribed to attentional bias and implicit appraisal has not yet been substantiated. Only a few studies have assessed attentional bias and negative implicit appraisal in genital pain, and these have reported contradictory results.

One study assessed hypervigilance for coital pain and a selective attentional bias towards pain stimuli in women with and without dyspareunia ${ }^{71}$. Questionnaires were used to assess hypervigilance, while the emotional Stroop task was used to assess attentional bias towards pain stimuli. Indications of hypervigilance for coital pain and also of a selective attentional bias towards pain stimuli were found in the genital pain group. This effect was predicted by state and trait anxiety and fear of pain ${ }^{71}$. Another study assessed the role of cognitive distraction from sexual stimuli in women with dyspareunia, women with low sexual desire and sexually functional women ${ }^{72}$. Erotic images were shown, each containing an object intended to distract from the erotic regions of the scene, while an eye tracker recorded the women's eye movements. 
Indications of cognitive distraction and an additional component of cognitive avoidance of sexual stimuli were found specifically in the female genital pain group ${ }^{72}$. To the best of our knowledge, no studies have investigated attentional bias towards sexual stimuli in women with vaginismus. So far, although evidence is scarce, the outcomes of the above two studies seem to confirm the assumed involvement of attentional bias towards sexual stimuli associated with penetration or other sexual stimuli. More studies are needed to further explore the role of attentional bias in female genital pain.

Regarding the involvement of negative implicit appraisal in female genital pain, one study explored implicit threat and disgust appraisal in women with vaginismus, women with dyspareunia and sexually functional women ${ }^{40}$. Two single-target Implicit Association Tasks (st-IAT) were used to measure implicit appraisals. In addition, uncontrollable physiological disgust responsivity was explored using facial electromyography (EMG). The women with dyspareunia and vaginismus both showed stronger implicit disgust appraisals of sexual stimuli compared to the control group of sexually functional women. The vaginismus group in particular showed enhanced levator activity as a unique physiological expression of disgust. No evidence was found for stronger implicit threat appraisals of sexual stimuli in the clinical groups ${ }^{40}$. Two studies assessed global affective associations in genital pain, using the Affective Simon Task. One study compared women with vaginismus, women with dyspareunia and sexually functional women ${ }^{41}$; the other study compared women with dyspareunia with sexually functional women ${ }^{39}$. In neither of the studies was any support found for the involvement of negative implicit global affective appraisals in genital pain. We may conclude that, although there are some indications of the involvement of implicit disgust appraisals in genital pain, there is no support for the involvement of stronger implicit threat appraisals in genital pain. Further studies are needed to substantiate the assumed role of implicit threat appraisals in genital pain.

Another remaining question that cannot be explained by the CB model is the reason why women may persist in having painful intercourse. One explanation for the different behaviour tendencies to either approach or avoid vaginal penetration could be that women with vaginismus and dyspareunia may differ in their attention for, and their implicit threat appraisals of, sexual stimuli. However, as described above, this has not been confirmed by previous evidence. Another explanation may be found in individual 
variables expressed in inter-individual differences in approach motivation towards vaginal penetration ${ }^{73,74}$. Depending on past histories of reward, persistence behaviour towards painful penetration may elicit stronger implicit incentive processes ("wanting") in women with dyspareunia than in women with vaginismus ${ }^{73,75}$. Thus far, no studies have compared women with vaginismus and dyspareunia with regard to differences in attention bias and implicit incentive appraisals of sexual penetration stimuli. This may help to understand differences in approach/avoidance behaviour towards vaginal penetration between these two groups of women.

There is also an urgent need to understand important processes of change, in order to tailor therapeutic interventions for genital pain and increase their effectivity. Although we know that therapist-aided exposure treatment is successful in reducing fear and pain catastrophizing cognitions in vaginismus, and this strongly supports the CB model, mechanisms of change have not yet been established.

A final issue that needs clarification is that, although the CB model ascribes an important role to sexual arousal during vaginal penetration in preventing and overcoming genital pain, hardly any studies have assessed the influence of sexual arousal on the appraisal of genital pain sensations. To the best of our knowledge, only three studies have assessed the influence of sexual arousal on genital pain sensitivity. Only one of these studies assessed pleasurable genital sensations as a function of sexual arousal. One study determined vaginal sensitivity ${ }^{76}$, while the two other studies focused on the sensitivity of the vulvar vestibule 54,77. The evidence so far seems to indicate that sexual arousal increases genital pain sensitivity ${ }^{54,77}$ and does not affect pleasurable sensitivity ${ }^{77}$, whereas the results for genital touch sensitivity have been contradictory, with either no change ${ }^{76,77}$ or increased sensitivity ${ }^{54}$ as a function of sexual arousal. These findings contradict the importance of the role of sexual arousal in preventing or overcoming genital pain 2,78 .

It is obvious that there are enough questions to be clarified in the cognitive processes relating to the aetiology and persistence of genital pain. 


\section{Aims and outline of the present thesis}

The aim of the research reported on in the current thesis was to explore which implicit and explicit cognitive and emotional processes underlie painful or pleasurable vaginal penetration in women and how these can be modified.

The studies presented in Chapters 2 and 3 aimed to substantiate the suggested role of attentional bias and implicit appraisals in genital pain. In view of the prominent role of fear in the CB model of genital pain, and the lack of evidence for the involvement of implicit threat, two of our studies assessed (implicit and explicit) threat appraisals of sexual stimuli in women with genital pain. In addition, we focused on the question whether increased attention bias for sex-related threats (e.g., pain) and reduced implicit incentive processes ("wanting") may play a role in the impairment of sexual arousal and the development of genital pain. We also explored differences in attention, implicit threat and incentive processing between women with vaginismus, women with dyspareunia and sexually functional women, with the aim of explaining differences in persistence or coital avoidance behaviour towards vaginal penetration between the two clinical groups (Chapter 2).

Chapter 3 reports on a study investigating the modifiability of (implicit and explicit) threat and negative general affective valences of erotic stimuli in women with vaginismus following therapist-aided exposure, and comparing this with a sexually functional group of women. This was done because general affective appraisals, as well as implicit appraisals, are assumed to be relatively more rigid and difficult to modify ${ }^{79-}$ 82.

Attentional bias was assessed using a Visual Search Task (VST, Chapter 2), as this task can differentiate between two relevant components of attention: enhanced vigilance and increased distraction ${ }^{83}$. The single-target Implicit Association Test (stIAT, Chapters 2 and 3) was used to measure implicit sex-threat and sex-wanting associations. The Affective Simon Task (AST, Chapter 3) was used to assess global affective associations. Clinical implications, as well as methodological arguments that may explain the results of the studies, are discussed in both chapters. 
The study described in Chapter 4 assessed whether the outcome of treatment with therapist-aided exposure was mediated by changes in positive and catastrophizing pain/ penetration cognitions. Although therapist-aided exposure has proved to be successful in reducing both fear and catastrophizing cognitions in vaginismus, psychological mechanisms of change have not yet been established. Participants with lifelong vaginismus were randomly allocated to a 3-month exposure condition or a waiting list control condition. Full intercourse was assessed daily for 12 weeks. Secondary outcome measures (complaints about vaginismus and coital pain) were assessed at baseline and after 12 weeks. Questionnaires were used to assess positive and catastrophizing penetration (pain) cognitions. Indications for clinical practice are discussed.

The studies described in Chapters 5 and 6 tested the role ascribed to sexual arousal in preventing and overcoming genital pain. Previous studies could not confirm a positive influence of sexual arousal on genital sensations. Since the available instruments lacked ecological validity, a new instrument was developed to simulate the vaginal sensations during penetration in a sexual context. Using the Vaginal Pressure Inducer (VPI), gradually increasing vaginal pressure was administered in a standardized and controlled manner in the introitus vaginae in a group of women without sexual complaints. Chapter 5 describes the first exploratory study with the VPI, with the aim of validating the instrument. Tolerance for and the level of painfulness/pleasurableness of vaginal pressure were tested in neutral, erotic and explicitly sexual contexts. In addition to the role of sexual arousal in the appraisal of genital sensations, the chapter discusses the results in the context of previously used assessments methods to test genital sensitivity.

In the study presented in Chapter 6 we explored the role of individual differences regarding the appraisal of genital pressure and a possible mediating role of pain and positive expectations as a function of sexual arousal. We induced gradually increasing vaginal pressure in sexually functional women in a neutral acclimatization condition, followed by a sexual and a non-sexual condition. Implications of the findings for the prevention and treatment of intercourse pain are considered.

Chapter 7 summarizes the main results, and discusses methodological and clinical considerations, as well as directions for future research. 



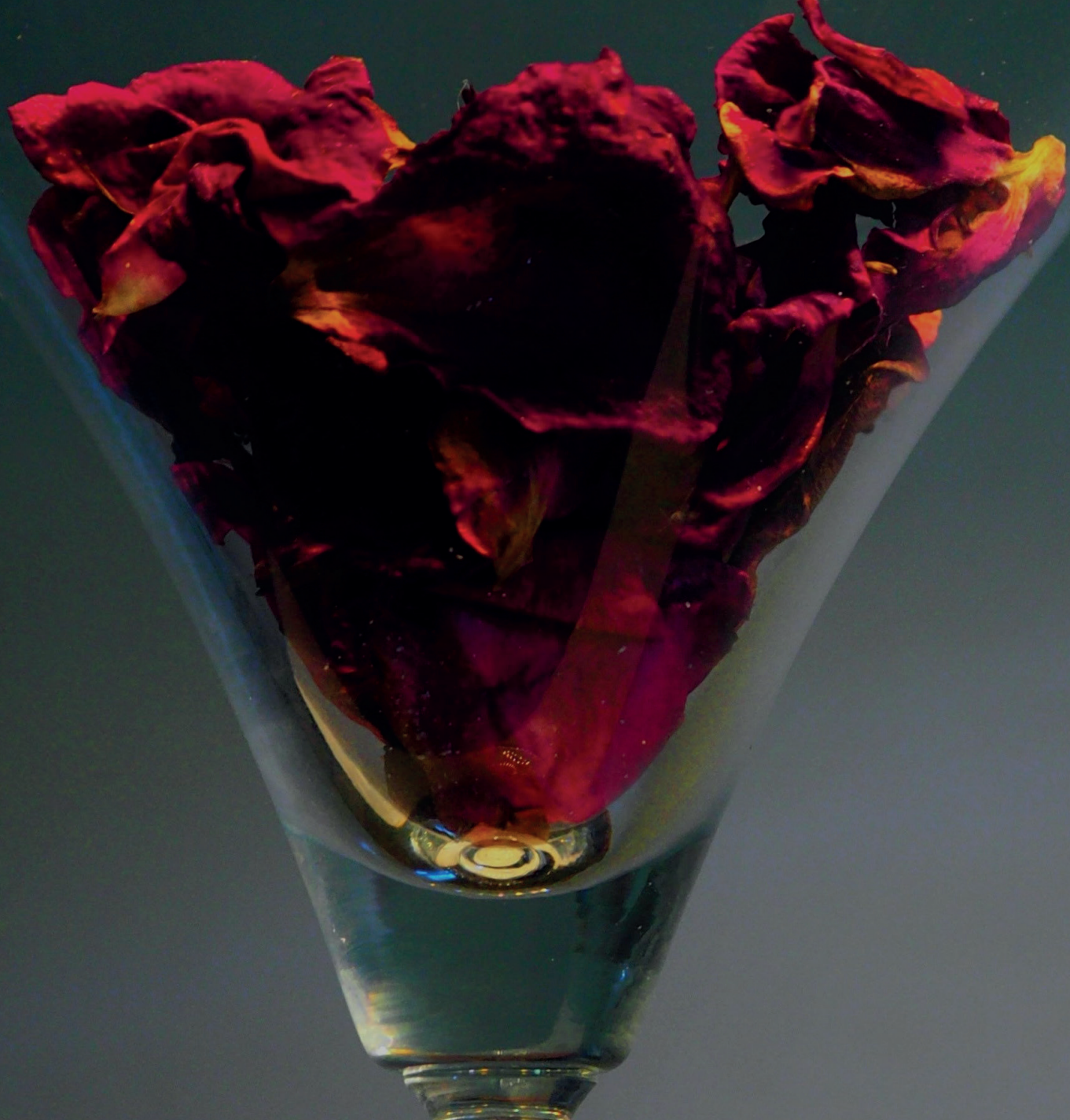

\section{Chapter 2}

Attentional Bias for Pain and Sex, and Automatic Appraisals of Sexual penetration: Differential patterns in Dyspareunia versus Vaginismus?

R. J. Melles, M. D. Dewitte, M. M. ter Kuile, M. L. Peters, and P. J. de Jong Journal of Sexual Medicine, 2016, 13, 1255-1262 


\section{ABSTRACT}

Introduction: Current information processing models propose that heightened attention bias for sex-related threats (e.g., pain) and lowered automatic incentive processes ("wanting") may play an important role in the impairment of sexual arousal and the development of sexual dysfunctions such as Genito-pelvic pain/penetration disorder (GPPPD). Differential threat and incentive processing may also help explain the stronger persistence of coital avoidance in women with vaginismus compared to women with dyspareunia.

Aims: As the first aim, we tested if women with GPPPD show (i) heightened attention for pain and sex, and (ii) heightened threat and lower incentive associations with sexual penetration. Second, we examined whether the stronger persistence of coital avoidance in vaginismus versus dyspareunia might be explained by a stronger attentional bias, or more dysfunctional automatic threat/incentive associations.

Methods: Women with lifelong vaginismus $(n=37)$, dyspareunia $(n=29)$, and a nosymptoms comparison group $(n=51)$ completed a Visual Search Task to assess attentional bias, and single target Implicit Association Tests to measure automatic sexthreat and sex-wanting associations.

Results: There were no group differences in attentional bias or automatic associations. Correlational analysis showed that slowed detection of sex stimuli and stronger automatic threat associations were related to lowered sexual arousal.

Conclusions: The findings do not corroborate the view that attentional bias for pain or sex contributes to coital pain, or that differences in coital avoidance may be explained by differences in attentional bias or automatic threat/incentive associations. However, the correlational findings are consistent with the view that automatic threat associations and impaired attention for sex stimuli may interfere with the generation of sexual arousal. 


\section{INTRODUCTION}

Genital pain is a common phenomenon in women, and a substantial proportion of women (20 - 47\%) report regular pain experiences during coitus ${ }^{84-86}$. Genital pain and fear of genital pain are also core symptoms of the DSM 5 defined Genito-pelvic pain/penetration disorder (GPPPD) ${ }^{9}$. Current information processing models of sexual arousal propose that attentional processes may be critically involved in the impairment of sexual arousal and the persistence of sexual problems such as genital pain 1,70,87. If attention is preferentially directed towards threatening aspects of having sex, this may impede the development of sexual arousal. Attempts of penile-vaginal penetration in the absence of sufficient arousal and lubrication may well result in genital pain. Repeated painful experiences may increase both fear of pain and vigilance for pain cues, leading to further impairement of sexual arousal 42,43. Consistent with the view that attentional bias for pain might be involved in GPPPD, women with dyspareunia showed relatively strong color naming interference effects when pain-related stimuli (e.g., burning, cutting) were presented in a modified Stroop task ${ }^{71}$. However, we cannot be sure that the interference effects during the Stroop task reflect processing priority; it may also reflect attempts to inhibit the processing of pain stimuli 88 or fear-induced rumination ${ }^{89}$. Therefore, the first aim of this study was to test further whether women with coital pain are indeed characterized by an attentional bias for pain-related stimuli in terms of faster detection and heightened distraction.

In this study we also measured attentional bias for sex stimuli. Because sex has frequently been paired with the experience of pain, sex stimuli may have acquired painsignalling properties in women with coital pain. Such acquired threat value may promote avoidance of sex-cues. Consistent with this view, women with dyspareunia showed relatively few eye fixations on sex-relevant details in a free viewing task 72 . Actively avoiding the processing of sexual cues (e.g., attention regulation) interferes with the generation of sexual arousal 87,90, which in turn may contribute to the development or persistence of GPPPD (e.g., ${ }^{72}$ 91). In line with the view that threat signals will elicit enhanced vigilance and increased distraction ${ }^{92}$, a visual probe study that focused on the initial more automatic allocation of attention, found that female students who reported relatively frequent experiences of coital pain and low sexual arousal 
showed a relatively strong attentional bias for verbal sex stimuli ${ }^{91}$. In the current study we tested the robustness of these earlier findings, and examined if women with GPPPD show a heightened attentional bias for sex stimuli. In addition, we examined whether we could replicate the earlier finding that attentional bias for sexual stimuli was associated with low sexual arousal.

We included both women with dyspareunia and women with lifelong vaginismus. Despite the recent integration of vaginismus and dyspareunia into a single diagnosis within the DSM-5, there are striking coping differences towards sexual penetration attempts between both groups. There is evidence indicating that the prospect of genital penetration elicits relatively strong fear-avoidance tendencies in women with vaginismus ${ }^{18}$, whereas women with dyspareunia show stronger task persistence in the context of painful intercourse 52 . These differences in avoidance/persistence tendencies towards penetration cues may be due to differences in sensitivity for signals of pain (attentional bias). Therefore, we tested whether women with dyspareunia are less vigilant for and less distracted by (signals of) pain than women with vaginismus.

In addition, vaginal penetration has been shown to be more strongly connected to threat in women with vaginismus than in women with dyspareunia 18 13. Therefore, we also tested if sexual penetration stimuli elicited weaker automatic threat associations in women with dyspareunia than in women with vaginismus. Finally, differences in penetration attempts between women with dyspareunia and women with vaginismus may relate to differences in approach motivation ${ }^{74}$. Sexual penetration stimuli may elicit stronger automatic incentive processes ${ }^{73,75}$ ("wanting") in women with dyspareunia than in women with vaginismus. Therefore, we also tested if women with dyspareunia show stronger automatic wanting associations with sexual penetration stimuli than women with vaginismus.

Together, we tested the following hypotheses: i) women with GPPPD show heightened attentional bias for pain stimuli relative to controls and the attentional bias for pain is especially pronounced in women with vaginismus; ii) women with GPPPD show heightened attentional bias for sex stimuli relative to controls, and the attentional bias for sex is especially pronounced in women with vaginismus; iii) women with vaginismus show stronger penetration-threat associations than women with dyspareunia; 
iv) women with dyspareunia show stronger automatic wanting associations towards sexual penetration stimuli than women with vaginismus; v) independent of diagnosis, lower sexual arousal will be associated with heightened attentional bias for both pain and sex, together with weaker sex-wanting and stronger penetration-threat associations.

\section{METHODS}

\section{Participants}

The patients were referred by a gynaecologist or general practitioner to the Regional Centre of Sexology from the Maastricht University Medical Centre. Inclusion criteria for the clinical group were lifelong vaginismus (never been able to engage in full intercourse, i.e. the penis never passed the pelvic floor muscles) or, for the dyspareunia group, genital pain in at least $50 \%$ of (attempted) vaginal penetrations for at least 3 months duration; age between 19 and 45 years, good command of the Dutch language, and a heterosexual relationship for at least 3 months. Participants were excluded in case of pregnancy, breast-feeding, major affective disorder, psychotic disorder, substance related disorder, posttraumatic stress disorder resulting from sexual abuse (according to DSM-IV-TR criteria ${ }^{10}$ ), which was used in the Netherlands at the time of the study, or a physical explanation for the sexual dysfunction. All women with dyspareunia/ vaginismus underwent a standardized physical examination 25 to exclude physical causes for the complaint. The diagnosis of lifelong vaginismus/ dyspareunia was assessed in the context of a full sexual history.

The control sample was recruited through advertisements at the university, in the local media, and by personal contact. Inclusion criteria for the control women were: absence of sexual problems, age between 19 and 45 years, good command of the Dutch language and a heterosexual relationship for at least 3 months. The study was approved by the medical ethical committee of the Maastricht University Medical Centre (P06-130). Part 
of the participating women with vaginismus and part of the control group also participated in another study ${ }^{93}$. The total study sample consisted of 117 participants: 37 women with vaginismus, 29 women with dyspareunia, and a control group of 51 participants and a control group of 61 participants without sexual complaints. Table 1 shows the demographic characteristics. There were no significant differences in terms of age, years of education or cohabitation-status between the groups.

Part of the (vaginismus and control) sample that participated in another study ${ }^{93}$ did not receive the single target Implicit Association Tests (st-IATs). Therefore, the analyses of the st-IAT results were restricted to a subsample of 82 participants (19 women with vaginismus (age $M=25.37$, standard deviation $[S d]=6.69$ ), 32 women with dyspareunia (age $M=25.84, S d=6.09$ ), and 31 control women (age $M=24.87$, $S d=4.48$ ). Participants received 20 euros as compensation for their participation in the study. 


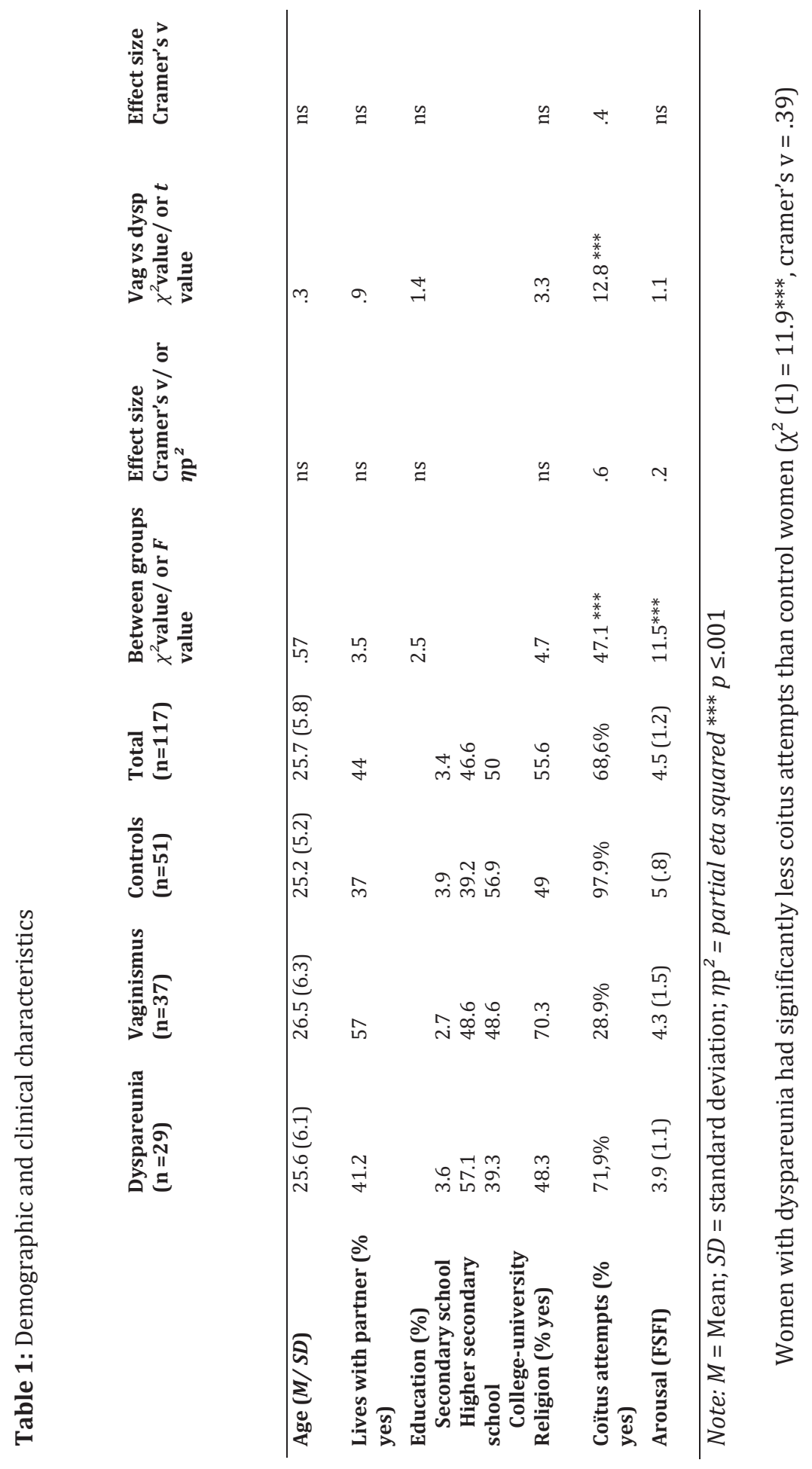




\section{Main outcome measures}

\section{Visual Search Task (VST)}

We used a Visual Search Task (VST) (cf. ${ }^{94}$ ) to measure attentional bias for pain and sexual stimuli. The VST can differentiate between two relevant components of attention: enhanced vigilance, evidenced by faster detection of relevant (pain/sex) cues, and heightened distraction ${ }^{83}$. Each trial started with a tone followed by a $500 \mathrm{~ms}$ fixation cross in the middle of the screen. Subsequently, participants were presented with a $5 \times 4$ matrix of 20 words. Participants had to indicate as fast as possible whether the matrix contained 20 words of the same category or contained one word from a different category by pressing the proper button of a response box.

For the Pain VST, three word categories were used: one pain-related (e.g., irritating, prickly, stinging etc.), and two neutral control categories: colour (green, purple, etc.) and nationality (Belgian, Spanish, etc.). Also for the Sex VST, three word categories were used: sexual words e.g., penetration, penis, arousal), and words from two types of neutral control categories: cooking (e.g., draining, kitchen) and sport (e.g., rugby, running).

Matrices on target-present trials consisted of one pain (or one sex) word among 19 neutral words, one neutral word among 19 pain (or 19 sex) words, one neutral word (e.g., nationality) among 19 neutral words from another neutral category (e.g., colours). Each of the six types of matrices was shown 19 times to each participant. The first 12 trials were presented as practice trials, followed by the test phase of 114 target-present trials and 30 target-absent trials. The function (i.e., target or distractor) of the neutral categories was counterbalanced over participants.

Because only the target-present trials were relevant for testing the hypotheses, the analyses were restricted to these trials. Errors (i.e., misses; pain: 8.7\%; sex: 6.4\%) and missing data (only in the pain task, $0.1 \%$ ) of the target-present trials, responses faster than $200 \mathrm{~ms}$ ( 1 trial in the pain task) and higher than three SD above the overall mean of the remaining response latencies were excluded (pain: 1.3\%; sex 0.9\%). False alarm rates to the target-absent trials were low (Pain VST: pain: 2.3\%; colour 4.7\%; country: $2.3 \%$; Sex VST: sex: 3.5\%; sport: 5.1\%; cook: 5.2\%). 


\section{Single Target Automatic Association task (st-IAT)}

The st-IAT is developed to assess to what extent a single target category (in this case, vaginal penetration) is associated with two attribute categories ${ }^{40,93}$. We used a "threat vs. safe" and a "wanting" vs. not wanting" st-IAT to assess the role of "threat" and "wanting" associations with sexual penetration. Target stimuli were pictures representing vaginal penetration. Attributes were "threat- vs. safe-related and "wanting" vs "not wanting"-related words. The stIAT started with 20 practice trials, followed by two test phases. Participants were instructed to sort sexual pictures and attribute words that appeared in the middle of a computer screen as fast as possible into the appropriate category by pressing either the $\mathrm{P}$ or $\mathrm{Q}$ keys on a keyboard. In one test phase, "sex pictures" and "threat" c.q. "not wanting" were mapped onto a single response key and "safe" c.q. "wanting" on the other. In the other test phase, this mapping was reversed. The target-attribute combinations that shared response keys were counterbalanced. Each critical test phase consisted of 60 trials in which responses were divided equally over the two response keys to prevent response bias.

The D-4 measure was computed 9596 on the trials of the test phases ${ }^{40,93}$. A negative Dmeasure reflected relatively strong threat and weak wanting associations.

\section{Female Sexual Function Index (FSFI)}

To index sexual arousal we used the total score of the four-item sexual arousal subscale of the FSFI 97,98 . Since the FSFI is only considered valid if the respondent has been sexually active in the preceding 4 weeks ${ }^{99}$, women who did not engage in sexual activity in the preceding 4 weeks were excluded from analyses involving the FSFI (4 women with vaginismus, 1 woman with dyspareunia). Cronbach`s $\alpha$ of the arousal subscale were satisfactory for all subgroups (vaginismus $\alpha=0.87$, dyspareunia $\alpha=0.73$, and controls $\alpha$ $=0.84)$. 


\section{Penetration Behavioral Questionnaire (PBQ)}

To index intercourse behavior during the last four weeks, the one item 'intercourse attempts' of the PBQ was used 57,58. The PBQ is rated on a 4-point scale: (a) not attempted, (b) attempted but unsuccessful, (c) attempted and sometimes successful, (d) attempted and always successful. Ratings were dichotomized with 0 reflecting not attempted (a) and 1 reflecting attempted $(a, b$, or $c)$.

\section{PROCEDURE}

After informed consent was obtained, participants received a personalised internet link to complete the questionnaires at home. Thereafter the lab session took place. To prevent undesirable priming effects on our measure of attentional bias, the pain and sex VSTs were presented first (jn counterbalanced order) After a 10 minutes break the experiment continued with the threat and wanting st-IATs (and two IATs that were unrelated to this study), also in counter-balanced order. We also included a group of participants who completed the VSTs in the context of another study (for details see ${ }^{93}$ ). These participants also started the lab-session with the VST but completed the questionnaires after the VST the during lab session. Comparing the subgroups revealed no significant differences in outcome variables (VST).

\section{Data Analyses}

To examine group differences in detection speed RTs on Target trials of the VST were subjected to a 3 (Group) x 2 (Target: Pain/Sex vs. Neutral) repeated measures ANOVA. Differences in distraction were tested with a 3 (Group) x 2 (Distractor: Pain/sex vs. Neutral) repeated measures ANOVA on Distractor trails. To assess group-differences in automatic sex apparaisal, we used multivariate ANOVAs with groups as independent 
variable and the D-4 measures of the threat and wanting st_IAT as dependent variable. For all analyses, effect sizes are reported using Partial Eta squared $\eta \mathrm{p}^{2} 100$.

Bivariate correlations were computed to evaluate the relationships between attentional bias, automatic associations and sexual arousal. Attentional bias scores were calculated by subtracting mean scores (RTs) of pain and sex VSTs from the mean scores (RTs) of the neutral VSTs. negative scores reflected slower detection/more distraction, positive scores reflected faster detection/ less distraction than neutral targets.

\section{RESULTS}

\section{Clinical characteristics}

As can be seen in Table 1, women with dyspareunia showed more coital attempts than women with vaginismus, but women with dyspareunia reported significantly less attempts than control women. Women with dyspareunia and vaginismus reported significantly lower sexual arousal than the control group.

\section{Attentional bias for sexual and pain stimuli (VSTs)}

Technical problems led to missing data in the Pain VST for 3 in the control group; in the Sex VST for 4 women in the vaginismus group, 1 in the dyspareunia group, and 3 in the control group. Table 2 shows the RTs for the various types of trials as a function of group. Main effects for Target Pain $F(1,110)=4.9, p=.029, \eta \mathrm{p}^{2}=0.04$ and Target Sex $F$ $(1,107)=20.78 p<.001, \eta \mathrm{p}^{2}=0.16$ were found, indicating that, overall, women were significantly faster to detect both pain and sex targets, than neutral targets in arrays of neutral stimuli. 
Main effects of Distractor Pain: $F(1,110)=6.8, p=.01, \eta \mathrm{p}^{2}=0.06$ and Distractor Sex: $F$ $(1,107)=31.25, p<.001, \eta p^{2}=0.23$ were found, indicating that participants were significantly faster to detect a neutral target in an array of pain stimuli, than in an array of neutral distractors. In a similar vein, participants were faster to detect a neutral target in an array of sex stimuli, than in an array of neutral distractors. These effects were comparable across groups, as evidenced by the absence of significant interactions with group, $F \leq \sum 1.11 ; p \geq .35 ; \eta \mathrm{p}^{2} \leq .02$.

\section{Implicit Threat/wanting associations (st-IAT)}

Technical problems (failure to save data) led to missing data for 5 women in the vaginismus group, 7 in the dyspareunia group, and 2 in the control group. The D-4 scores are presented in Table 2. For the sex-wanting task the st-IAT-index was overall larger than zero as evidenced by a significant intercept $\mathrm{F}(1,65)=8.44, p \leq .01, \eta \mathrm{p}^{2}=$ 0.12 , indicating that women had overall stronger automatic wanting than not-wanting associations with vaginal penetration pictures. There was no significant main effect of Group $\mathrm{F}(2,65)=0.22, p=.8, \eta \mathrm{p}^{2}=.01$, indicating that the automatic wanting associations were similar for all groups. For the sex-threat st-IAT, overall the index did not significantly differ from zero $\mathrm{F}(1,65)=0.03 p=.86, \eta \mathrm{p}^{2}=.001$. In addition, the index was similar across groups as evidenced by the absence of a main effect of Group F $(2,65)=1.21 p=.31, \eta \mathrm{p}^{2}=.04$. 
Table 2: Outcome VST and st-IAT tasks

\begin{tabular}{lllll} 
VST & $\begin{array}{l}\text { Reaction times } \\
\text { Dyspareunia } \\
M(S D)\end{array}$ & $\begin{array}{l}\text { Vaginismus } \\
\mathrm{M}(S D)\end{array}$ & $\begin{array}{l}\text { Controls } \\
M(S D)\end{array}$ & $\begin{array}{l}\text { Total } \\
M(S D)\end{array}$ \\
\hline Pain VST & $(\mathrm{n}=29)$ & $(\mathrm{n}=37)$ & $(\mathrm{n}=48)$ & $(\mathrm{n}=114)$ \\
t pain & $4341(671.16)$ & $4257.08(879.25)$ & $4033,06(669,79)$ & $4184.1(749.84)$ \\
t/d no pain & $4361,74(590,17)$ & $4368,05(706.08)$ & $4209,67(700,91)$ & $4299.15(674.54)$ \\
d pain & $4230.45(714.76)$ & $4363,3(714,76)$ & $4007,81(687,89)$ & $4179.82(728.08)$ \\
& & & & \\
Sex VST & $(\mathrm{n}=28)$ & $(\mathrm{n}=33)$ & $(\mathrm{n}=48)$ & $(\mathrm{n}=110)$ \\
t sex & $4172.02(791.93)$ & $4102,35(891,89)$ & $3951,1(727,44)$ & $4050.89(793.83)$ \\
t/d no sex & $4342,09(934,8)$ & $4430,13(837,43)$ & $4303,59(753,55)$ & $4350.65(818.38)$ \\
d sex & $4111.97(837.16)$ & $4044,55(789,06)$ & $3841,54(645,2)$ & $3968.96(742.83)$
\end{tabular}

st-IAT D-4 measures

\begin{tabular}{lllll} 
& $(\mathrm{n}=25)$ & $(\mathrm{n}=14)$ & $(\mathrm{n}=29)$ & $(\mathrm{n}=68)$ \\
threat & $.04(.23)$ & $-.10(.37)$ & $.04(.32)$ & $.01(.30)$ \\
wanting & $.29(.49)$ & $.21(68)$ & $.18(.70)$ & $.23(.61)$ \\
\hline
\end{tabular}

Note: VST = Visual Search Task (VST); $\mathrm{t}=$ target, $\mathrm{d}=$ distractor; St-IAT = single target Implicit Association Test; ${ }^{* *} \mathrm{p} \leq .01,{ }^{*} \mathrm{p} \leq .05 ; M=$ Mean, $S D=$ standard deviation

\section{Relationships between attention and automatic threat and wanting associations and sexual arousal}

Low sexual arousal was related to slow detection of sex stimuli and relatively strong automatic sex-threat appraisals. Automatic sex-wanting associations appeared unrelated to sexual arousal scores. Furthermore, the D-4 scores of the two st-IATs correlated positively with each other, indicating that the stronger the automatic sexthreat associations the weaker the sex-wanting associations. No relationships were found between attentional bias for pain or sex and automatic associations. 
Table 3: Relationships of attention, implicit appraisals, genital pain and sexual arousal

$\begin{array}{llllll}\text { VST } & \text { VST } & \text { VST } & \text { VST } & \text { st-IAT } & \text { st-IAT } \\ \text { t-Pain } & \text { d-Pain } & \text { t-Sex } & \text { d-Sex } & \text { Threat } & \text { Wanting }\end{array}$

\begin{tabular}{|c|c|c|c|c|c|c|}
\hline t-Pain $(n=113)$ & & & & & & \\
\hline d-Pain (n = 113) & $.43^{* *}$ & & & & & \\
\hline$t-\operatorname{Sex}(n=107)$ & .03 & .08 & & & & \\
\hline$d-\operatorname{Sex}(n=107)$ & .02 & .1 & $.65^{* *}$ & & & \\
\hline $\begin{array}{l}\text { st-IAT Threat } \\
\qquad(\mathrm{n}=62)\end{array}$ & .16 & -.13 & -.1 & -.02 & & \\
\hline $\begin{array}{l}\text { st-IAT Wanting } \\
\qquad(\mathrm{n}=63)\end{array}$ & .21 & .08 & .19 & .14 & $.31^{*}$ & \\
\hline $\begin{array}{l}\text { Genital Pain } \\
\qquad(\mathrm{n}=118)\end{array}$ & -.11 & .03 & .05 & -.04 & -.05 & .07 \\
\hline Arousal $(n=123)$ & -.05 & -.01 & $.17^{*}$ & .04 & $.25^{*}$ & .07 \\
\hline
\end{tabular}

\section{DISCUSSION}

This study examined attentional bias for pain and sex together with automatic sexwanting and sex-threat associations in the context of GPPPD. The main findings can be summarized as follows: (i) women generally showed an attentional bias for pain and sex as indexed by both faster detection and heightened distraction; (ii) women generally showed stronger automatic sex/wanting than sex/not-wanting associations, whereas their sex/threat and sex/safe associations were of similar strength; (iii) the strength of the attentional biases and automatic sex-associations were similar for all groups; (iv) overall, low sexual arousal was associated with slow detection of sex-relevant target words and strong sex-threat associations. 


\section{Attentional bias}

Women were generally faster to detect pain and sex stimuli than neutral stimuli. This response pattern was similar for all groups and thus, the hypothesis that women with GPPPD are characterized by a facilitated detection of pain or sex stimuli and/or heightened distraction by pain or sex stimuli was not supported.

These findings seem inconsistent with earlier work showing an attentional bias for painrelated words in women with dyspareunia ${ }^{71}$. However, this earlier study used the Emotional Stroop task (ES) and several authors have challenged the validity of the ES as an index of attentional bias (e.g., 88,89. The current failure to find an heightened attentional bias for pain stimuli in women with GPPPD parallels the failure to find a robust attentional bias towards pain-related words or pictures in chronic pain patients 101. So far, attentional bias has mainly been indexed by visual tasks. Yet, the visual dimension might not be most critical in the context of pain. To further examine the relevance of attentional bias in GPPPD it might be important for future research to use tactile (e.g., genital pressure) instead of visual stimuli.

Interestingly, the correlational analyses across groups showed that slowed detection of sex stimuli was related to lower levels of sexual arousal. Thus consistent with the information processing model of sexual arousal ${ }^{1}$ this finding suggests that impaired attention for sex may hinder the generation of sexual arousal. To arrive at more final conclusions it would be important to replicate the current findings preferably in studies using multiple indices of attentional bias ${ }^{90}$.

\section{Automatic associations}

The current study showed an overall relationship between relatively strong automatic sex-threat associations and relatively low levels of sexual arousal. Previous studies already showed that self-reported threat appraisals may interfere with the generation of sexual arousal 60,68, the current study is the first to show that also more automatic sexthreat associations are associated with low sexual arousal 40,93. To modify low sexual 
arousal it may be important to target both the more deliberate and the more automatic threat appraisals (cf. ${ }^{102}$ ).

In line with previous research 40,93 , the current study failed to find evidence for heightened automatic penetration-threat associations in women with GPPPD. Together the available findings suggest that in women with GPPPD, the automatic responses may have kept a predominantly sexual meaning; possibly protected by the reproduction value of sex, positive reinforcement of sexual responses, confirmation of the relationship, etc.. Such an interpretation is also in line with the consistent finding that although women with GPPPD do report impaired levels of subjective sexual arousal, they do not show lower levels of genital arousal towards sexual penetration pictures than women without sexual problems $60,67,68$. Thus the available evidence seems to converge to the conclusion that especially the more explicit, subjective negative appraisals are involved in GPPPD.

The current study was the first to assess automatic sex-wanting associations in women with GPPPD. The findings indicated that women generally showed an automatic motivational orientation towards sexual penetration. The findings failed to support the hypothesis that women with dyspareunia show stronger automatic motivational associations with sexual penetration stimuli than women with vaginismus. Thus relatively weak sex-wanting associations seem not involved in the avoidance of penetration attempts in women with vaginismus. It should be acknowledged that the sex-wanting associations were assessed in a non-individualized laboratory context, using generic pictures of sexual penetration. Because automatic associations in women have been found to be highly context-dependent 103, it cannot be ruled out that automatic wanting associations in women with vaginismus are hampered in the context of actual sexual behaviors and heightened sexual arousal.

Interestingly, relatively strong automatic sex-threat associations were associated with relatively weak automatic sex-wanting associations. This is in line with the view that a strong threat value of sex may interfere with individuals' readiness of having sex and hamper the generation of sexual arousal. However, it should be acknowledged that these findings are purely correlational thus neither the direction of the relationship nor the causal properties of the automatic associations can be derived from the current findings. 


\section{Limitations}

The sex stimuli and the pain stimuli that were used might not be a proper conceptual representation of the relevant features that women with GPPPD actually experience during sex. Therefore, it remains to be seen whether comparable results will occur in real life contexts by influence of the presence of the partner and by highlighting the emotional consequences of sexual (dis)functioning. A challenge for future research is to develop more ideographic stimuli which may help to improve the external validity of laboratory research.

With respect to the automatic associations, the group of women with vaginismus was very small $(n=14)$ thereby lowering the sensitivity of the current study to find group differences; however, since the differences between groups were very small, these negative findings seem not merely attributable to a lack of statistical power. Although the current findings suggest that dysfunctional automatic attention and automatic appraisal processes are not critically involved in GPPPD, further research with automatic tasks with higher external ecological validity is needed to arrive at more solid conclusions in this respect.

\section{Conclusions}

The findings of the present study did not support the view that attentional bias for pain or sex is involved in GPPPD, nor that relatively strong automatic threat and/or relatively weak wanting associations with sexual penetration play an important role in the generation and/or maintenance of dyspareunia and/or vaginismus. In terms of clinical implications, we can conclude that explicit, subjective negative appraisals are the most promising targets for interventions aiming at reducing genital pain and vaginal penetration problems. To increase sexual arousal it may be helpful to encourage women to direct more attention to sexual stimuli and to modify the automatic threat appraisals 104-106. 



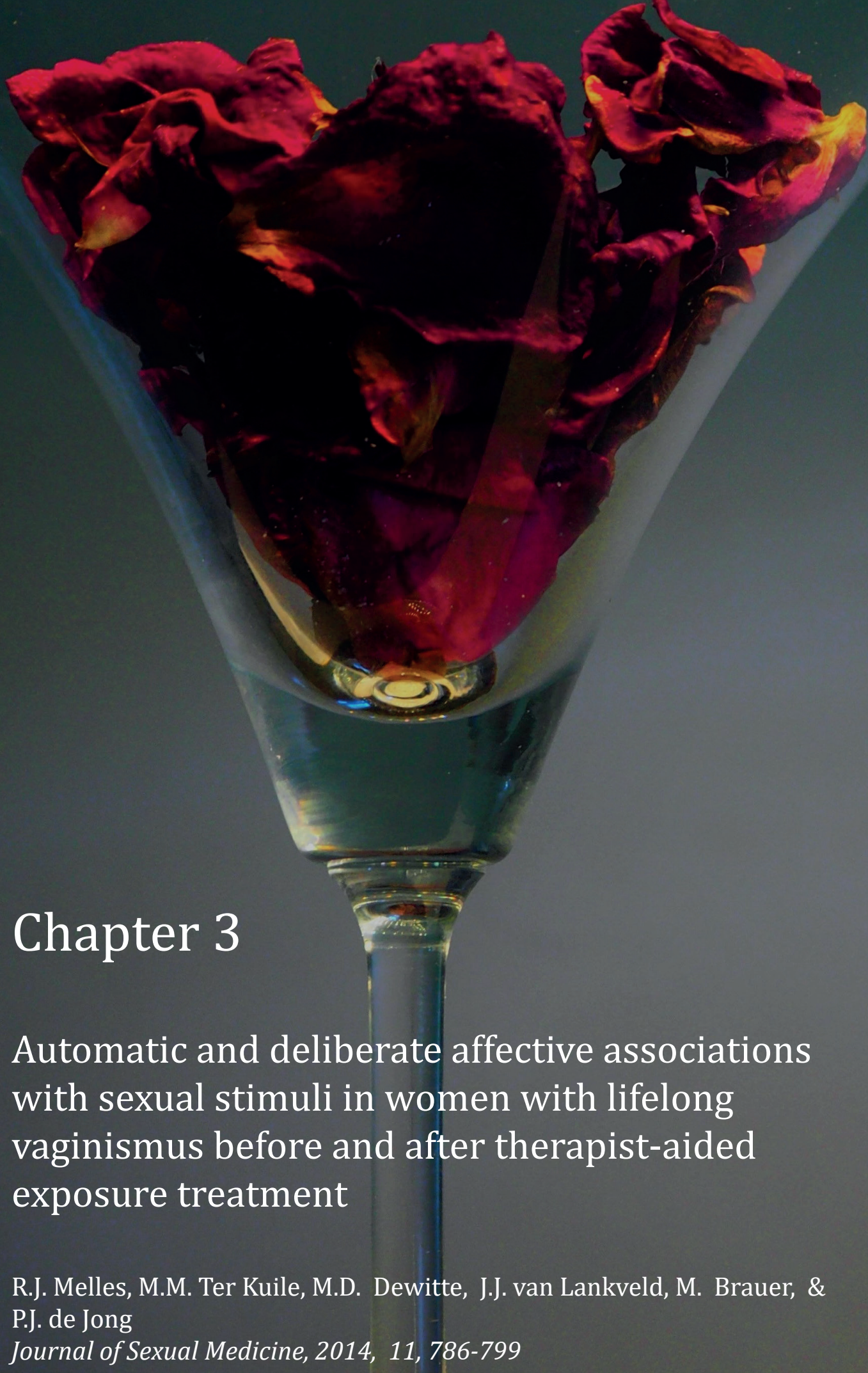




\section{ABSTRACT}

Introduction: The intense fear response to vaginal penetration in women with lifelong vaginismus, who have never been able to experience coitus, may reflect negative automatic and deliberate appraisals of vaginal penetration stimuli which might be modified by exposure treatment.

Aims: To examine whether in women with vaginismus (i) sexual stimuli elicit relatively strong automatic and deliberate threat associations, as well as relatively negative automatic and deliberate global affective associations, compared to symptom-free women; and (ii) these automatic and more deliberate attitudes can be modified by therapist-aided exposure treatment.

Methods: A single target Implicit Association Test (st-IAT) was used to index automatic threat associations, and an Affective Simon Task (AST) to index global automatic affective associations. Participants were women with lifelong vaginismus ( $\mathrm{N}=68)$, and women without sexual problems $(\mathrm{N}=70)$. The vaginismus group was randomly allocated to treatment $(n=34)$ and a waiting list control condition $(n=34)$.

Main Outcome Measures: Indices of automatic threat (st-IAT) and automatic global affective associations (AST),Visual Analogue Scales (VAS) to assess deliberate appraisals of the sexual pictures (fear, global positive affect).

Results: More deliberate fear and less global positive affective associations with sexual stimuli were found in women with vaginismus. Following therapist-aided exposure treatment, the strength of fear was strongly reduced, while global positive affective associations were strengthened. Automatic associations did not differ between women with and without vaginismus and did not change following treatment.

Conclusions: Relatively stronger negative (threat/global affect) associations with sexual stimuli in vaginismus appeared restricted to the deliberate level. Therapist-aided exposure treatment was effective in reducing subjective fear of sexual penetration stimuli and led to more global positive affective associations with sexual stimuli. The impact of exposure might be further improved by strengthening the association between vaginal penetration and positive affect (e.g., by using counter-conditioning techniques). 


\section{INTRODUCTION}

In the Diagnostic and Statistical Manual of Mental Disorders, 4th edition, text revision (DSM IV-TR) vaginismus was defined as an involuntary contraction of the musculature of the outer third of the vagina interfering with intercourse, causing distress and interpersonal difficulty ${ }^{10}$. Results of empirical studies did, however, not support the main criterion of vaginal spasm as a critical feature of vaginismus and emphasized the role of fear/pain and phobic avoidance as factors that interfere with the declared wish of the women to experience sexual intercourse ${ }^{78}$. Due to persistent difficulties in differentiating, vaginismus and dyspareunia have been combined in the DSM-5 into 'Genito-pelvic pain/penetration disorder" 9. Vaginismus can be classified as lifelong or acquired. Women with lifelong vaginismus have never been able to experience coitus. In acquired vaginismus women lose the ability to experience coitus after a period of nonsymptomatic functioning. Pain, fear of pain, and pelvic floor dysfunction are common in the majority of women with vaginismus and make it difficult to distinguish vaginismus from other sexual pain disorders, specifically with dyspareunia 13,17,25,35,49,78,107,108. Yet, there is a marked difference between women with vaginismus and women with dyspareunia regarding their response to pelvic examination: In these contexts women with vaginismus show more intense fear responses (e.g. fear of pain) as well as heightened defensive and avoidant distress behavior, despite the declared wish to be able to allow penetration. During pelvic examinations some patients may cry, withdraw from the examiner and/or try to leave the examination room probably all due to the intense fear that is elicited at the prospect of any form of vaginal penetration ${ }^{13}$. The first aim of the present study is to investigate whether the intense fear response in women with vaginismus is elicited by automatic and deliberate threat appraisals of sexual (penetration) stimuli.

There is evidence that (the anticipation of) fearful occurrences give rise to involuntary contractions of the pelvic floor muscles. This response is considered as part of a general defense mechanism (e.g., ${ }^{24}$ ). Interestingly, there is evidence that the anticipation of pain or harm associated with vaginal penetration can similarly elicit defensive contractions in women with vaginismus ${ }^{48}$, which in turn may lead to increased genital pain experiences. All in all, it seems that fear- avoidance mechanisms are crucially involved in 
vaginismus. Following this, it has been argued that vaginismus might be conceptualised as a specific phobia of vaginal penetration ${ }^{109}$. To the extent that the vaginistic reaction indeed reflects a (vaginal penetration) fear induced defensive reflex, prolonged exposure to vaginal penetration stimuli, and the consequent extinction of the fear response, would be helpful to reduce the vaginistic symptoms ${ }^{110}$. In support of this theoretical model, exposure therapy was found to be very effective in reducing symptoms of vaginismus ${ }^{59}$. Thus, there is converging evidence that phobic avoidance plays a key role in the maintenance of vaginismus.

Current cognitive models of anxiety emphasize the role of biased appraisals in the generation of phobic avoidance (e.g., 111). Dual-process models 82,111,112 and informationprocessing models of sexual responding ${ }^{1}$ emphasize the importance of differentiating between more automatic (initial) and more deliberate reflective appraisals. The initial appraisals are proposed to follow from the direct activation of simple associations in memory, whereas the more deliberate appraisals are assumed to be the result of more controlled, reflective processes. The encounter with vaginal penetration stimuli will thus elicit a relatively fast, nonintentional (automatic) affective appraisal, followed by a slower deliberate appraisal. The more automatic appraisals seem especially relevant in guiding relatively spontaneous (reflexive) behaviors, whereas the more deliberate appraisals seem most important for more controlled behaviors (e.g., 113,114). Therefore, it seems reasonable to assume that especially the more automatically activated affective appraisals of particular sexual (penetration) stimuli are most relevant in eliciting the characteristic defensive reactions in women suffering from vaginismus, whereas more deliberate threat appraisals might give rise to more controllable defensive behaviours such as overt avoidance of vaginal penetration.

Germane to this, previous research provided tentative support for the view that both more deliberate (e.g. ${ }^{13,109}$ ) and more automatic negative ${ }^{40}$ appraisals might be involved in vaginismus. However, it should be noted that the difference in automatic sex-threat associations between women with vaginismus and women without sexual problems did not reach statistical significance in the latter, relatively small scale, study. To arrive at more solid conclusions regarding the relevance of automatic threat associations in vaginismus it is therefore important to replicate this previous finding in a larger sample. 
By now there is considerable evidence that therapist-aided exposure treatment is successful in reducing subjective fear of penetration and vaginal penetration avoidance which is operationalized during the graded exposure in guiding the patient to induce fingers, dilators, and tampons vaginally ${ }^{58,59}$. However, it remains to be seen whether the more automatic threat appraisals are also affected by such intervention, especially when considering that automatic associations are assumed to be more rigid than deliberate reflective evaluations ${ }^{80-82}$. Therefore, the present study is designed to further investigate the relevance of automatic versus deliberate threat associations with sexual stimuli in vaginismus, and to test whether these associations are malleable by treatment 115 .

Although previous research showed that exposure in vivo is effective in reducing the perceived threat of sexual intercourse, it appeared not successful in resolving impaired sexual pleasure 57-59,109. One explanation for this pattern of findings could be, that even though exposure does eliminate (deliberate) threat-associations with vaginal penetration stimuli (e.g., by the experience of having enough space to introduce the penis, by reappraising the sensation of penetration as an unknown feeling instead of painful), the intervention might not be successful in modifying the more global negative evaluation of vaginal penetration stimuli, i.e. introitus of the erect penis, or of erotic stimuli in general. Germane to this, research has shown that extinction procedures (i.e., presenting the conditioned stimulus (in case of vaginismus: vaginal penetration) without a contingent unconditional stimulus (pain) are highly successful in reducing catastrophic expectancies upon presentation of the conditioned stimulus (and thereby in reducing the signal-value of the conditioned stimulus, as a predictor of pain/harm, thereby diminishing fear), but far less in modifying the evaluative response towards the stimulus, the more general negative affective valence of the conditioned stimulus ${ }^{116}$. This fits with the more general observation that global affective associations are relatively resistant to extinction ${ }^{79}$. Perhaps, then, also in the context of vaginismus sexual (penetration) stimuli might elicit more global negative associations that are relatively resistant to extinction. In other words: being able to experience vaginal penetration following exposure treatment, might not imply positive associations with coitus. 
In line with this, previous research has shown that women with vaginismus reported more negative global affective appraisals of vaginal penetration than women with dyspareunia or women without sexual dysfunctions ${ }^{41}$. These global negative appraisals appeared restricted to the more deliberate level. In contrast with the proposed role of negative automatic appraisals in vaginismus, research failed to find evidence for the presence of relatively strong automatic global negative appraisals of vaginal penetration in women suffering from vaginismus ${ }^{41}$. To improve the sensitivity of the implicit task (as a measure of automatic appraisals), in the present study the original AST was used with a voice key in which the participant had to respond with either saying "positive" or "negative" depending on the task-relevant feature. The idea behind the task is that the emotional content will be processed automatically, and will therefore moderate women's response time. When there is a match between response requirement and automatic affect, respondents will be fast, whereas the automatic affective response tendency will lead to slower reaction times (RTs) when there is a mismatch between response requirement and the emotional content. Using the voice key might theoretically improve the sensitivity, due to the intrinsic relationship between the required response (the word "positive") and a particular affective evaluation ${ }^{41}$, instead of learning the valence of the required response via sharing one response button with highly negative pictures and the other with highly positive pictures. Accordingly, as a second aim, this study will examine whether global negative automatic and deliberate associations with vaginal penetration and erotic stimuli might be involved in vaginismus and whether this type of affective associations is indeed relatively resistant to extinction by exposure treatment.

\section{Aims}

The aim of this study is to investigate whether automatic threat associations and more global negative associations with vaginal penetration stimuli are involved in vaginismus. Accordingly, this study examined whether women with vaginismus are indeed characterised by (i) relatively strong (automatic) threat associations with vaginal penetration, and/or (ii) relatively negative global (automatic and deliberate) affective associations with vaginal penetration and erotic stimuli, and (iii) whether these associations are modified by exposure treatment. 


\section{METHODS}

\section{Participants}

The members of the symptomatic group were women who participated in a treatment outcome study ${ }^{59}$. Inclusion criteria for the treatment outcome study were lifelong vaginismus, of at least 6 month's duration, age between 18 and 65 years, good command of the Dutch language, a heterosexual relationship for at least 3 months and willingness of the partner to participate in the treatment sessions. The diagnosis of lifelong vaginismus was assessed in the context of a full sexual history. The lifelong nature of the complaint was judged by the assessor (master student clinical psychology), based on the applicant's self-report." Participants were excluded if they had had full sexual intercourse at any time, by exploring, in case of doubts, if, to the opinion of the women, the penis had ever passed her pelvic floor muscles. Further exclusion criteria were suffering from major affective disorder, psychotic disorder, substance related disorder, posttraumatic stress disorder resulting from abuse in the area of the pelvic floor and the genitals (e.g., sexual assault) according to DSM-IV-TR criteria ${ }^{10}$; or a physical reason for the inability to have intercourse (congenital hymeneal or vaginal abnormalities). All women completed questionnaires, followed by a standardized gynecological examination (only for women with vaginismus) to exclude any physical causes for their symptoms. The procedure of the standardized gynecological examination consisted of visual examination of the vulva and a cotton swap test, and is described in more detail elsewhere ${ }^{59}$. The selection procedure of the present study was comparable with the procedure used in our previous studies 57,58. Participants in the treatment outcome study were invited to also participate in the current study. One of the 70 women in the outcome study refused to participate in the current study, because of religious reasons.

An age-matched control sample was recruited through advertisements at the university, in the local media and by personal contact. Inclusion criteria were: absence of sexual problems, age between 18 and 65 years, good command of the Dutch language and a heterosexual relationship for at least 3 months. The control participants received 15 euros as a compensation after having completed the study. 
One participant had difficulties with comprehending the task instructions, even after repeated instructions. Her data of both computer tasks were identified as outliers $(\mathrm{z}>$ 3). Consequently, it was decided to discard these data. Regarding the control AST, the data of two cases were discarded, one in the vaginismus group and one in the control group, due to outlier RTs ( $>$ > 3). Our final study sample consisted of 68 women with lifelong vaginismus and 70 control women. Table 1 presents participants' biographic and complaints characteristics.

Table 1: Demographic variables

\begin{tabular}{lllll} 
& $\begin{array}{l}\text { Vaginismus } \\
\text { Exposure }\end{array}$ & Waiting list & Total & Controls \\
& $\mathrm{n}=34$ & $\mathrm{n}=34$ & $\mathrm{n}=68$ & $\mathrm{n}=70$ \\
\hline Age $(M / S D)$ & $28.24(7.73)$ & $29.29(7.03)$ & $28.76(7.35)$ & $29.20(8.71)$ \\
Education (\%) & & & & \\
$\quad \begin{array}{l}\text { Secondary school. } \\
\text { Higher secondary school }\end{array}$ & 17.3 & 32.4 & 33.8 & 21.5 \\
$\quad$ College-university & 47.1 & 14.7 & 16.2 & 25.7 \\
$\begin{array}{l}\text { Duration of complaint } \\
\text { (years) } M(S D)\end{array}$ & $9.49(6.54)$ & $11.49(6.61)$ & $10.49(6.54)$ & \\
Lives with partner (\% yes) & 76.5 & 76.5 & 76.5 & 58.9 \\
\hline
\end{tabular}

Note: $M=$ Mean, $S D=$ standard deviation

Part two of the study is part of a randomized controlled trial. To investigate the effect of exposure treatment, women of the vaginismus group were randomly assigned to a Study Condition: Treatment Condition (TC) or a waiting list condition (WLC). Both groups completed pre-treatment and 12-weeks post-treatment assessments. After the posttreatment assessment, the participants in the WLC also received the active treatment followed by post-treatment assessment. Assessment was performed at each research centre by two research assistants who were not involved in treatment delivery and who were (at pre-assessment) blinded to the study condition. Approval for the study was obtained from the medical ethical committees of both hospitals and was carried out according to its standard procedures. The procedure of the therapist-aided exposure treatment is described elsewhere 58,59 . 


\section{Main outcome measures}

\section{Single Target Implicit Association task (st-IAT)}

The st-IAT is a computerized reaction time (RT) task developed to measure to what extent a single target category (in this case, vaginal penetration) is associated with two attribute categories (threatening vs. safe) 40,41,117. The st-IAT has already been applied successfully in previous research to test implicit threat-appraisals of sexual stimuli ${ }^{40}$. Pictures representing vaginal penetration formed the stimuli for the (single) target. The stimuli for the attributes contained threat- vs. safety-related words ${ }^{40}$.

There were two test phases (60 trials each), that were both preceded by a practice phase (20 trials each). Women were instructed to sort sex pictures and stimulus words as fast as possible with as few mistakes as possible into the appropriate category (for the pictures "sex" and for the attributes "threatening" or "safe") using the P and Q keys on a keyboard. In one test phase, "sex" and "threat" were mapped on to a single response key and "safe" on the other. In the other test phase, "sex" and "safe" were mapped together and "threat" on the other. The assumption is that the task becomes easier (and the responses faster) when two categories that are associated in memory share the same response key. The difference in RTs between both test phases is assumed to reflect whether penetration is associated more strongly with threat or safety.

During the task, the labels of the categories ("threat" / "safe") allocated to the left and right keys were presented in the upper left and right corners of the screen. Following a correct response, the stimulus was immediately replaced by a fixation dot in the middle of the screen, which was replaced by the next stimulus after 500 milliseconds (ms). Following an incorrect response, the Dutch word "FOUT" (false) appeared shortly above the stimulus. The stimulus remained on the screen until the correct response was given. The target-attribute combinations that shared response keys were counterbalanced (i.e., block order). Each critical test block consisted of 60 test trials in which responses were divided equally over the two response keys. 


\section{Deliberate Fear Associations with Sexual Stimuli (st-IAT)}

To assess deliberate fear associations with the target sex stimuli women were asked to rate each erotic picture on a Visual Analogue Scales (VASs), ranging from 0 (not at all) to 100 (very). In addition to the fear VAS, three other VASs were offered to measure the dimensions disgust, pleasure, and unpleasant. Since these dimensions do not directly apply to the current research questions, these data are not reported in this paper.

\section{Affective Simon task}

Automatic global affective associations with sexual (penetration and non-penetration) stimuli were obtained using a pictorial version of the AST 39,118. The AST consisted of three phases. The first priming phase is to strengthen the bond between the required response and the affective valence of the picture (e.g., ${ }^{119}$ ). In this phase women were asked to say "positive" when the picture had a "positive" valence and "negative" when the picture had a "negative" valence. Six positive pictures and six negative pictures were selected from the International Affective System (IAPS) (Numbers of positive pictures: $1441,1750,2050,5760,8496,8501$; numbers of negative pictures: 2205, 2750, 9001, $9421,9440,9830)^{120}$. All pictures were presented twice in portrait format and twice in landscape format. Hence, the priming phase comprised of 48 trials.

During phases 2 and 3, women were instructed to respond as fast as possible with saying either "positive" or "negative" to indicate whether the picture was presented in landscape or portrait format. Prior to the sex-AST, women were presented with 12 practice trials with neutral pictures selected from the IAPS to become familiar with the procedure (Numbers of neutral pictures: 7004, 7006, 7009, 7010, 7035, 7080).

In the second phase, i.e. the sex-AST, women were presented with 12 target pictures, namely six sexual (penis-vagina) penetration pictures and six heterosexual nonpenetration pictures (kissing, cunnilingus, fellatio and manual stimulation of the vulva). The sexual pictures were selected shots taken from a women-friendly erotic film ${ }^{121}$ One size fits all, directed by Candida Royalle ${ }^{39}$. All pictures were presented eight times in landscape and eight times in portrait format. In total, 192 trials were presented. To 
control for possible stimulus order effects, the erotic pictures were presented in two mirrored fixed random stimulus presentation orders ${ }^{39}$. During the third phase, i.e. the control-AST, 6 pictures with a positive and 6 with a negative emotional valence, selected from the IAPS were offered (Numbers of positive pictures: 1440, 1710, 2222, 2388, 2550, 7580; numbers of negative pictures: 2900, 6610, 9300, 9404, 9800, 9911). All pictures were presented 3 times in landscape and 3 times in portrait format. Hence, the control-AST consisted of 72 trials. The control-AST was included to control the validity of the present experimental set-up and to examine whether any between group effects on the "sex-AST" might be attributed specifically to the sexual stimuli.

The response requirements were the same during the second and third phase, and were counterbalanced across participants. To prevent women from focusing on one point of the screen, the dimensions of the offered pictures altered randomly in 5 different reshapes ${ }^{39}$. Women were placed in front of the computer screen at a distance of circa 40 $\mathrm{cm}$. Each phase was introduced with specific instructions on the computer screen. It was emphasized to respond as fast as possible with as few mistakes as possible to minimize any effects of deliberate stimulus associations. The voice-key recorded reaction times. The female research assistant recorded inaccurate responses and voice-key failures. Each trial consisted of a fixation cross (500 ms) followed by a stimulus. The RTs indicated the level of congruency of the emotional valence of the pictures and the required response ("positive" or "negative"). More information regarding the pictorial sex-AST used in this study can be found elsewhere ${ }^{39}$.

\section{Deliberate Global Affective Associations with Sexual Stimuli}

To index deliberate global affective associations with vaginal penetration and erotic stimuli, the women were asked to rate each of the target pictures they had seen previously in the sex-AST on a Visual Analogue Scale (VAS), with the left anchor being "negative" (0) and the right anchor being "positive" (100). 


\section{PROCEDURE}

After general instructions on the experimental procedure were given and informed consent was obtained, a sexual history was taken, followed by the Mini-International Neuropsychiatric Interview Plus (M.I.N.I. Plus) ${ }^{122,123}$ to check inclusion criteria. The stIAT and the AST were taken in a sound-attenuated room, in counter-balanced order. Finally, the women with vaginismus were randomly allocated to the TC or WLC. Postassessment took place after 3 months. The first assessment took about 3 hours and the 3-months post-assessment took about 1 hour. Twenty women also participated in another study, described elsewhere.

\section{Data reduction}

In line with previous research, st-IAT effects were computed on the basis of the attribute trials only ${ }^{40}$. The so-called D-4 measure was computed on the basis of the test-trials according to the commonly used scoring algorithm ${ }^{95}$ which recently had been shown to perform best in the current measurement setting ${ }^{96}$. All RTs above 10,000 ms were discarded. Error trials were replaced with the mean RT of the correct responses of the pertinent block plus a penalty of $600 \mathrm{~ms}$. The st-IAT effects were calculated such that a negative D-measure reflected relatively strong associations with threat ${ }^{40}$. To compute mean RTs of the AST, all error trials and all RTs below $200 \mathrm{~ms}$ and longer than $3000 \mathrm{~ms}$ were discarded. Unfortunately, many zero RTs were observed, due to voice key problems in the AST. Considering the reliability of the mean RTs it was decided to discard the mean RTs if the proportion of zero trials was $>40 \%$, leading to missing AST data in $20,6 \%$ of the vaginistic group and in $27,9 \%$ of the control group.

Data of the AST were LN transformed to normalize the positively skewed distribution. 


\section{RESULTS}

\section{The cross-sectional part of the study}

Figure 1 shows the number of missing data and drop-outs, of the women with lifelong vaginismus and the reasons for dropping out. Of 70 control women, accurate st-IAT data sets were obtained of 65 participants and accurate AST data sets of 51 participants.

\section{The intervention part of the study}

Six of 68 women (9\%) left the study before the 12-weeks assessment (see Figure 1). Ttests for independent samples revealed no differences between dropouts and followthrough women $(p<.05)$ on demographic or pre-treatment data (st-IAT and AST).

\section{Automatic threat associations in women with vaginismus and controls (st-IAT)}

To calculate group differences in automatic threat associations, the D-measures were subjected to independent $t$-tests. Effect sizes were calculated with the Cohen's d (difference mean scores/ SD pooled) 124 . For the purpose of interpretation, Cohen considered $0.2<d<0.5=$ small; $0.5<d<0.8=$ medium $d>.8=$ large ${ }^{125}$. No significant difference in automatic threat associations were found between women with vaginismus and control women, $t(127)=0.49, p>.05, d=0.09$ (vaginismus group $n=64, M=-0.09$, $S D=0.62$, control group $n=65, M=-0.14, S D=0.51$ ). A one-sample $t$-test showed that, overall, women had stronger automatic threat associations with vaginal penetration pictures, compared with zero, i.e. automatic neutral associations, $t(128), t=-2.30, p<$ $.05, d=0.41$. 


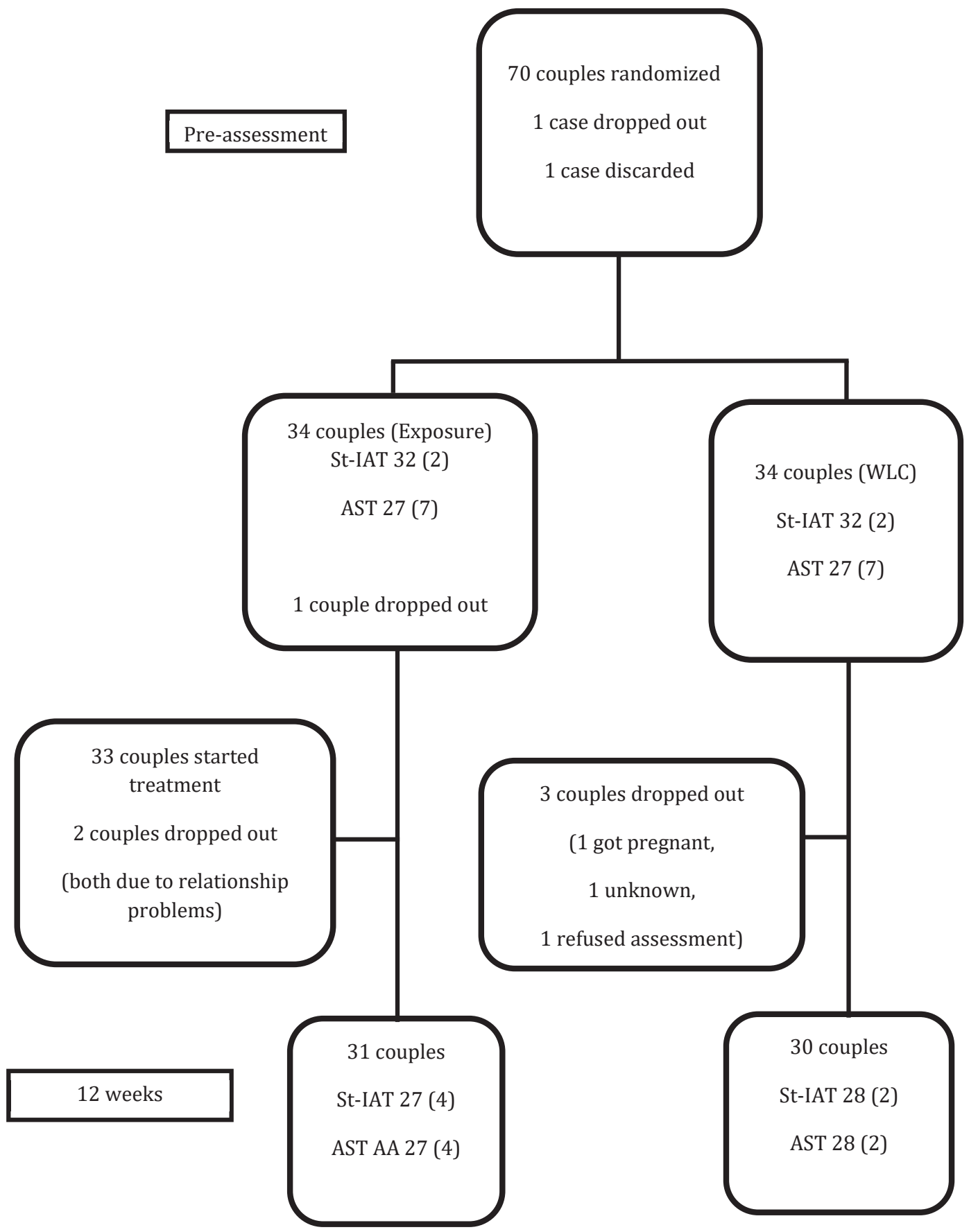




\section{Deliberate fear associations in women with vaginismus and controls (st-IAT)}

To test group differences in deliberate threat associations, VAS fear ratings of the target stimuli presented during the st-IAT were subjected to independent $t$-tests. Vaginal penetration stimuli elicited significantly stronger deliberate fear associations in women with vaginismus $(n=64, M=35.19, S D=27.90)$ than in control women $(n=65, M=$ $10.74, S D=15.69), t(99)=6.12, p<.01, d=1.08$.

\section{Automatic Global Negative Associations in Women with Vaginismus and Controls (Sex-AST)}

To investigate whether women with vaginismus showed more global negative automatic associations than control women, we entered mean RTs of the sex-AST as dependent variables in a 2 Group (vaginismus, controls) x 2 Stimulus Category (penetration, nonpenetration) x 2 Required Response (positive, negative) ANOVA with the latter two factors as within-subject factors. Effect sizes were calculated with the Partial Eta small; $0.06<\eta \mathrm{p}^{2}<0.14=$ medium $\eta \mathrm{p}^{2}>0.14=$ large 126.

A main effect of Required Response was found: $F(1,103)=13.08 p \leq .01, \eta p^{2}=0.11$ indicating that, overall, participants were relatively fast when the required response was positive (see Table 2). This effect (positive implicit attitude towards sex stimuli) seemed similar for both groups, based on the absence of a Required Response by Group interaction, $F(1,103)=0.03, p>0.5, \eta \mathrm{p}^{2}<0.01$. This AST-effect was most pronounced for penetration stimuli, regarding the Required Response by Stimulus Category interaction, $F(1,103)=4.45, p<.05, \eta p^{2}=.04$. This pattern seemed similar for both groups based on the absence of Required Response x Stimulus x Group interaction, $F$ (1, 103) $=0.07, p>0.5, \eta p^{2}<0.01$. Unrelated to the main research question, two significant main effects were found; there was a main effect of Group $F(1,103)=7.98, p<.01, \eta \mathrm{p}^{2}$ $=0.07$, reflecting that women with vaginismus were, overall, slower reacting than the control group; (see Table 2), and a main effect of Stimulus Category $F(1,103)=15.16, p$ 
$<.01, \eta p^{2}=0.13$, which indicates that, overall, participants responded faster to penetration pictures, $t(105)=-3.923, p<.01, d=-0.77$. We repeated these analyses on the first part of the sex-AST (instead of the means of two sex blocks) to control for the potential influence of learning effects of exposure to sex stimuli (desensitize) which might result in less automatic associations to sex stimuli. These analyses did not reveal significant interaction effects either.

Table 2: Mean reaction times for Stimulus Category and Required Response in the sex-AST

\begin{tabular}{lll}
$\begin{array}{l}\text { Required response } \\
\text { Sexual stimuli }\end{array}$ & $\begin{array}{l}\text { Positive } \\
M(S D)\end{array}$ & $\begin{array}{l}\text { Negative } \\
M(S D)\end{array}$ \\
\hline Penetration & $722(167)$ & $758(234)$ \\
Vaginismus $(\mathrm{n}=54)$ & $640(165)$ & $664(160)$ \\
Controls $(\mathrm{n}=51)$ & $682(171)$ & $712(206)$ \\
Combined $(\mathrm{N}=105)$ & & \\
Nonpenetration & $734(185)$ & $783(245)$ \\
Vaginismus $(\mathrm{n}=54)$ & $642(148)$ & $688(191)$ \\
Controls $(\mathrm{n}=51)$ & $689(174)$ & $736(224)$ \\
Combined $(\mathrm{N}=105)$ & & $771(236)$ \\
Sex (total) & $728(174)$ & $667(171)$ \\
Vaginismus $(\mathrm{n}=54)$ & $639(157)$ & $721(213)$ \\
Controls $(\mathrm{n}=51)$ & $686(171)$ & \\
Combined $(\mathrm{N}=105)$ &
\end{tabular}

Note AST scores are shown in untransformed mean reaction times in milliseconds.. AST = Affective Simon Task; $M=$ mean; $S D=$ standard deviation 


\section{Control-AST}

To examine whether any between-group effects on the sex-AST might be attributed specifically to the sexual stimuli, we entered mean RTs of the control-AST as dependent variables in a 2 Group (vaginismus, controls) x 2 Emotional Valence (positive, negative) x 2 Required Response (positive, negative) ANOVA. Again a significant main effect of Group was found, $F(1,100)=4.88, p<.05, \eta \mathrm{p}^{2}=0.05$, indicating that, also for the nonsexual affective stimuli, women with vaginismus were, overall, slower in their responding than the control group (see Table 3). In addition, a main effect of Required Response was found, $F(1,100)=10.82, p<.01, \eta \mathrm{p}^{2}=0.10$, that was qualified by a Stimulus Valence $\mathrm{x}$ Required Response interaction, $F(1,100)=13.45, p<.01, \eta \mathrm{p}^{2}=$ 0.12. Attesting to the validity of the current AST, this interaction reflects that participants were generally faster when there was a match between the emotional valence of the picture and the valence of the required response. This influence of required response was especially pronounced for positive pictures, $t(101)=-4.78, p<$ $.01, d=0.95$, whereas it failed to reach significance for negative pictures, $t(102)=-0.95$, $p>.05 d=0.19$.

Table 3: Mean reaction times for Stimulus Category and Required Response in the control AST

$\begin{array}{lll}\text { Required response } & \text { Positive } & \text { Negative }\end{array}$

General affective stimuli $\quad M(S D) \quad M(S D)$

Positive

Vaginismus $(\mathrm{n}=52)$

$720(163)$

767 (151)

Controls $(n=50)$

$659(168)$

717 (207)

Combined $(\mathrm{N}=102)$

$690(167)$

$743(182)$

Negative

Vaginismus $(\mathrm{n}=52)$

737 (159)

750 (147)

Controls $(n=50)$

$684(168)$

$688(181)$

Combined $(\mathrm{N}=102)$

$711(164)$

$720(167)$

Note: AST scores are shown in untransformed mean reaction times in milliseconds;

AST = Affective Simon Task; $M=$ mean; $S D=$ standard deviation 
Table 4: Deliberate Global and Sexual Affective Associations of the AST Ratings

\begin{tabular}{llll} 
Global affect & Penetration & Nonpenetration & Sex (total) \\
& $M(S D)$ & $M(S D)$ & $M(S D)$ \\
\hline Vaginismus $(\mathrm{n}=54)$ & $38.98(27.78)$ & $50.39(26.29)$ & $44.69(25.15)$ \\
Controls $(\mathrm{n}=51)$ & $68.59(20.78)$ & $68.93(18.53)$ & $68.76(18.48)$ \\
Combined & $53.36(28.67)$ & $59.39(24.58)$ & $56.38(25.15)$
\end{tabular}

Note: Ranch for each descriptor 0-100. Higher scores indicate more positive affect; AST = Affective Simon Task; $M=$ Mean, $S D=$ standard deviation

\section{Deliberate Global Positive Affective Associations in Women with Vaginismus and Controls}

To test whether women with vaginismus reported less positive deliberate global affective responses to erotic stimuli, a 2 Group (vaginismus, controls) x 2 Stimulus Category (penetration, non-penetration) ANOVA was conducted on the VAS affect ratings of the stimuli presented in the AST. For global positive affect we found a significant main effect of Group $F(1,103)=30.95, p<.001, \eta p^{2}=0.23$, that was qualified by a Group x Stimulus Category interaction, $F(1,103)=10.95, p=.001, \eta p^{2}=0.10$. To interpret this interaction we conducted follow-up tests, revealing that both penetration stimuli, $t(98)=-6.21, p<.001, d=-1.25)$ and non-penetration stimuli, $t(95)=-4.20, p$ $<.001, d=-0.82$ ) elicited less positive affect in women with vaginismus than in control women. Only women with vaginismus reported less positive affect to penetration stimuli than to non-penetration stimuli, $t(53)=-4.21, p<.001, d=-0.57$, whereas in the control group no differences in reported affect values to the different Stimulus Categories were found, $t(50)=-0.17, p>.05, d=-0.03$. 


\section{Automatic Threat Associations (st-IAT) Pre- and Post-Treatment.}

To investigate pre-to post-treatment changes in automatic threat ratings we entered the D-measure (st-IAT) as dependent variable into a 2 Condition (TC, WLC) x 2 Time (pre-, post- treatment) ANOVA, with the last factor as a within-subject factor. No significant interaction between Time and Condition was found, $F(1,50)=0.12, p>.05, \eta p^{2}=0.01$, meaning that exposure treatment did not modify the automatic threat associations with vaginal penetration stimuli. The main effect of Time was not significant either, $F(1,50)$ $=0.13, p>.05, \eta \mathrm{p}^{2}<0.01$. Hence, no evidence was found that test-retest effects played an important role in participant's st-IAT performance (see also Table 5).

Table 5: Effect of exposure on automatic (D 600 measure) and deliberate threat associations (st IAT)

\begin{tabular}{lll} 
& $\begin{array}{l}\text { Pretreatment } \\
\text { Ratings }\end{array}$ & $\begin{array}{l}\text { Posttreatment } \\
M(S D)\end{array}$ \\
\hline Automatic threat & \\
Exposure (n=25) & $-0.18(0.61)$ & $-0.10(0.60)$ \\
WLC (n=27) & $-0.03(0.67)$ & $-0.03(0.54)$ \\
Total $(\mathrm{n}=52)$ & $-0.10(0.64)$ & $-0.06(0.57)$ \\
Deliberate threat & & \\
Exposure (n=25). & $30.98(31.88)$ & $20.07(19.24)$ \\
WLC (n=27) & $37.53(25.03)$ & $41.77(25.99)$ \\
Total $(\mathrm{n}=52)$ & $34.38(28.44)$ & $31.34(25.26)$
\end{tabular}

Note: For automatic threat: Higher negative scores means stronger threat-associations.

For deliberate threat: Higher scores indicate more threat $M=$ Mean, $S D=$ standard deviation; stIAT $=$ single target Implicit Association Test; $\mathrm{WLC}=$ waiting list condition . 


\section{Deliberate Fear Associations Pre- and Post-Treatment}

To investigate pre-to post-treatment changes in deliberate fear ratings, we entered the VAS fear ratings as dependent variables into a 2 Condition (TC, WLC) x 2 Time (pre-, post-assessment) ANOVA with Time as within-subjects factor. A Significant Time $\mathrm{x}$ Condition interaction effect was found, $F(1,50)=7.99, p<.01, \eta p^{2}=0.14$. Subsequent paired $t$-tests showed that this interaction reflected a significant decrease in deliberate fear ratings with regard to vaginal penetration stimuli after exposure treatment, $t(24)=$ 2.23, $p<.05, d=0.51$, whereas fear ratings did not change after the waiting period, $t$ (26) $-1.71, p>.05, d=-0.33$ (see also Table 5).

\section{Automatic Global Affective Associations (Sex-AST) Pre- and Post-Treatment}

To assess changes in automatic global affective associations, we entered the AST-data as dependent variables into a 2 Condition (TC, WLC) x 2 Time (pre-, post-assessment) x 2 Stimulus Category (penetration, non-penetration) x 2 Required Response (positive, negative) ANOVA, with the latter two factors as within-subject factors. The interactions between Condition x Time x Required Response and between Condition x Time x Required Response $x$ Stimulus Category did not reach significance, $F(1,40)=0.84, p>$ $.05, \eta \mathrm{p}^{2}=0.02$ and $F(1,40)=1.59, p>.05, \eta \mathrm{p}^{2}=0.04$, respectively. Thus there was no evidence indicating that exposure affected the automatic global affective associations with sexual stimuli. Only the main effect of Time was significant, $F(1,40)=14.24, p<$ $.01, \eta p^{2}=0.26$. As can be seen in Table 6 , this outcome reflects that participants were generally faster at post-assessment (probably reflecting a learning effect). This effect was similar for both types of stimuli, as evidenced by the absence of a significant interaction of Time $\mathrm{x}$ Stimulus Category, $F(1,40)=2.02, p>.05, \eta \mathrm{p}^{2}=0.05$. 
Table 6: Effect of exposure on mean reaction times for Stimulus Category and Required Response ( sex-AST)

\section{Pretreatment}

\begin{tabular}{lllll}
$\begin{array}{l}\text { Required Response } \\
\text { Sexual stimuli }\end{array}$ & $\begin{array}{l}\text { Positive } \\
M(S D)\end{array}$ & $\begin{array}{l}\text { Negative } \\
M(S D)\end{array}$ & $\begin{array}{l}\text { Positive } \\
M(S D)\end{array}$ & $\begin{array}{c}\text { Negative } \\
M(S D)\end{array}$ \\
\hline Penetration & & & & $683(149)$ \\
$\quad$ Exposure $(n=20)$ & $723(153)$ & $753(243)$ & $683(137)$ \\
WLC $(n=22)$ & $713(175)$ & $752(243)$ & $622(114)$ & $689(144)$ \\
Combined $(n=42)$ & $718(163)$ & $752(199)$ & $651(134)$ & $686(139)$
\end{tabular}

\section{Non-penetration}

$\begin{array}{lllll}\text { Exposure }(n=20) & 753(181) & 767(219) & 682(133) & 697(140) \\ \text { WLC }(\mathrm{n}=22) & 713(187) & 782(183) & 624(125) & 698(161) \\ \text { Combined }(n=42) & 732(183) & 775(198) & 652(131) & 698(150)\end{array}$

Sex (total)

$\begin{array}{lllll}\text { Exposure }(\mathrm{n}=20) & 738(165) & 760(227) & 682(140) & 690(137) \\ \text { WLC }(\mathrm{n}=22) & 713(179) & 767(167) & 623(118) & 694(151) \\ \text { Combined }(n=42) & 725(171) & 764(196) & 651(131) & 692(143)\end{array}$

Note: AST scores are showed in untransformed mean reaction times in milliseconds.

AST= Affective Simon Task; $M=$ Mean, $S D=$ standard deviation; $\mathrm{WLC}=$ Waiting List Condition

Table 7: Effect of exposure on deliberate Global and Sexual Affective Associations of the AST

\begin{tabular}{|c|c|c|c|c|c|c|}
\hline \multirow{3}{*}{ Ratings } & \multicolumn{3}{|c|}{ Pretreatment } & \multicolumn{3}{|c|}{ Posttreatment } \\
\hline & Penetration & $\begin{array}{l}\text { Non } \\
\text { penetration }\end{array}$ & $\begin{array}{l}\text { Sex } \\
\text { (Total) }\end{array}$ & Penetration & $\begin{array}{l}\text { Non } \\
\text { penetration }\end{array}$ & $\begin{array}{l}\text { Sex } \\
\text { (Total) }\end{array}$ \\
\hline & $M(S D)$ & $M(S D)$ & $M(S D)$ & $M(S D)$ & $M(S D)$ & $M(S D)$ \\
\hline Global affect & & & & & & \\
\hline $\begin{array}{l}\text { Exposure } \\
(n=25)\end{array}$ & $\begin{array}{l}35.63 \\
(25.47)\end{array}$ & $\begin{array}{l}45.58 \\
(26.27)\end{array}$ & $\begin{array}{l}40.60 \\
(23.90)\end{array}$ & $\begin{array}{l}51.63 \\
(25.17)\end{array}$ & $\begin{array}{l}55.38 \\
(25.69)\end{array}$ & $\begin{array}{l}53.50 \\
(23.48)\end{array}$ \\
\hline $\begin{array}{l}\text { WLC } \\
(n=28)\end{array}$ & $\begin{array}{l}39.31 \\
(28.12)\end{array}$ & $\begin{array}{l}50.70 \\
(24.38)\end{array}$ & $\begin{array}{l}45.01 \\
(24.33)\end{array}$ & $\begin{array}{l}40.72 \\
(28.43)\end{array}$ & $\begin{array}{l}53.49 \\
(25.19)\end{array}$ & $\begin{array}{l}47.10 \\
(25.03)\end{array}$ \\
\hline $\begin{array}{l}\text { Combined } \\
(n=53)\end{array}$ & $\begin{array}{l}37.57 \\
(26.71)\end{array}$ & $\begin{array}{l}48.29 \\
(25.17)\end{array}$ & $\begin{array}{l}42.93 \\
(24.00)\end{array}$ & $\begin{array}{l}45.87 \\
(27.25)\end{array}$ & $\begin{array}{l}54.38 \\
(25.20)\end{array}$ & $\begin{array}{l}50.12 \\
(24.29)\end{array}$ \\
\hline
\end{tabular}

Note: AST scores are showed in untransformed mean reaction times in milliseconds;

AST= Affective Simon Task; $M=$ Mean, $S D$ = standard deviation; WLC = Waiting List Condition 


\section{Deliberate Global Affective Associations Pre- and Post- Treatment.}

Ratings reflecting global affective associations were entered into a 2 Condition (TC, WLC) x 2 Time (pre-, post-treatment) ANOVA with Time as within-subject factor. For global affect we found a significant main effect of Time $F(1,51)=14.71, p<.01, \eta \mathrm{p}^{2}=$ 0.22 , that was qualified by a Condition $\mathrm{x}$ Time interaction effect, $F(1,51)=7.64, p<.01$, $\eta \mathrm{p}^{2}=0.13$. This differential effect seemed to be most pronounced for penetration stimuli, as evidenced by the Stimulus Category x Condition x Time interaction that just failed to reach the conventional level of significance, $F(1,51)=3.59, p=.06, \eta p^{2}=0.07$. Subsequent $t$-tests showed that in the treatment condition, the global affective evaluation became more positive for both the penetration, $t(24)=-5.29, p<.01, d=-$ .1.05, and the non-penetration stimuli, $t(24)=-4.31, p<.01, d=-0.89$; whereas in the WLC no significant changes in global affective evaluations of (penetration and nonpenetration) sex-stimuli were observed. In addition, there was a main effect of Stimulus $F(1,51)=14.06, p<.01, \eta \mathrm{p}^{2}=0.22$, indicating that, overall, penetration stimuli elicited less positive affective associations than non-penetration stimuli.

\section{DISCUSSION}

The main results of this study can be summarized as follows: (i) Women with vaginismus showed relatively stronger fear associations with sexual stimuli at the subjective (deliberate) level, but not at the more automatic level. (ii) Women with vaginismus also showed less positive self-reported global affective associations with sexual stimuli, whereas at the automatic level they showed similarly positive global affective associations with sexual stimuli as women without vaginismus. (iii) Exposure treatment was effective in reducing the subjective fear associations and in amplifying the self-reported global positive affective associations with sexual stimuli, whereas the automatic associations remained unaffected. 


\section{Fear-associations}

The current finding that women with vaginismus rated vaginal penetration stimuli as much more threatening than women of the control group, supports the alleged importance of fear in vaginismus. Consistent with the view that fear-associations are involved in the maintenance of vaginismus, these relatively strong fear-associations with vaginal penetration stimuli reduced in strength as a result of therapist-aided exposure treatment. Specifically, women in the treated condition showed a reduction in fearassociations with the penetration stimuli following treatment. However, self-reported levels of deliberate fear in response to vaginal penetration stimuli following exposure treatment, remained elevated compared to complaint-free women $(d=0.51)$. These findings concur with the self-reported levels of fear during penetration with the partner: After exposure treatment only half of the participants reported comparable levels of fear during penetration (with the partner) as the symptom-free control group, whereas the other participants still reported elevated levels of fear of penetration ${ }^{59}$. The findings of both studies indicate that there is ample room for further improvement.

Results from the st-IAT suggest that, at the more automatic level, for both women with and women without vaginismus, pictures of vaginal penetration elicit threatening rather than safe associations. However, consistent with previous research using a similar task 40 , no evidence emerged to indicate that these automatic sex-threat associations were relatively strong for women with vaginismus compared with control women. The failure to find differences between women with and without vaginismus seems not attributable to lack of statistical power (effect size of the between group contrast was very small and if anything in the opposite direction from what was expected).

Elaborating on the present findings, the more automatic threat associations with the vaginal penetration pictures in both groups seem to represent a more general phenomenon that is not restricted to women with vaginismus. These automatic threat associations with the vaginal penetration pictures in both dysfunctional and functional women may be explained by the explicit character of the (penis-vagina) penetration pictures which might refer to a threatening value, for example the risk of transmission of sexual diseases ${ }^{127}$ or sexual violence ${ }^{23}$. In the absence of group differences, it seems not surprising that the more automatic threat associations remained unaffected by exposure 
treatment. Together, the available evidence suggests that vaginal penetration stimuli initially elicit threatening appraisals in both functional and dysfunctional women, whereas specifically the women with vaginismus continue to assign threat value to penetration stimuli during the subsequent validation process. Thus the critical difference between women with and without vaginismus seems to reside in the more deliberate rather than the more automatic processing of explicit vaginal penetration stimuli. This suggests that subjective affect plays also a crucial role in the sexual system, in which negative subjective affect in sexual situations might cause serious suffering in women. It should be acknowledged, however, that although the present findings provided no evidence for relatively strong automatic threat associations in vaginismus, it might still be that other types of dysfunctional automatic associations do differentiate between women with and without vaginismus. Germane to this, it has been found that women with vaginismus distinguish themselves from controls in stronger automatic sex- disgust associations ${ }^{40}$. Based on the current study it cannot be ruled out that more specific sex-disgust associations might play a role in complaints of vaginismus see also 127. It would, therefore, be important for future research to further explore the relevance of such more specific associations and to examine their sensitivity to (exposure) treatment.

\section{Global affective associations}

In accordance with earlier research the current findings show that women with vaginismus also distinguish themselves from controls with regard to their subjective (deliberate) global affective associations with sexual stimuli ${ }^{41}$. These findings underline the important role of ambivalent emotions in response to erotic stimuli in women with various sexual dysfunctions 46,60,67. The current study in women with vaginismus showed that enhanced levels of more negative affect occurred not only in response to penetration stimuli, but also following non-penetration stimuli. These findings are consistent with the outcome of a recent study in which more negative subjective responses were found following scenes of intercourse, but, specific in women with vaginismus, also in response to erotic films ${ }^{68}$. These findings underline the suggestion of a more generally enhanced dispositional disgust propensity in women with 
vaginismus $^{128}$. Importantly, these global affective appraisals became more positive following exposure treatment. There is, however, still room for further improvement as the global affective associations with sex stimuli remained less positive than those of the non-symptomatic women. The fact that affective evaluations of sex stimuli did not reach the positive levels of the control group, might partially explain why sexual functioning remained impaired after exposure treatment ${ }^{57-59,109}$.

Replicating previous research that used the EAST instead of the AST ${ }^{41}$, the present study showed that, independent of sexual complaints, participants were generally faster when the required response was "positive" than when the required response was "negative", indicating that both women with and women without vaginismus are characterized by positive rather than negative automatic affective associations with sexual stimuli. Thus, when participants were primed to evaluate the stimuli on the dimension positivenegative (instead of threatening-safe as was the case for the st-IAT) positive associations appeared most prominent. The strength of the positive automatic associations was similar for stimuli depicting heterosexual acts without any form of penetration, and stimuli depicting explicit vaginal penetration. One explanation for the absence of a differential response pattern between women with and without vaginismus on the sexAST could be that the task lacked sufficient sensitivity to detect relevant automatic associations. However, individuals' reaction times during the sex-AST were clearly affected by the task-irrelevant picture content, suggesting its sensitivity as an indirect measure of affect: Participants were systematically faster when the required response was positive than when the required response was negative. In further support of the validity of the present task, the results of the control-AST clearly showed a general affective Simon effect with regard to universal positive/negative pictures. Thus sustaining the validity of the present task, participants were generally faster when there was a match than when there was a mismatch between the valence of the irrelevant feature (i.e., picture content positive or negative) and the valence of the required response ("positive" or "negative"). These results clearly demonstrate that the valence of the pictures interfered with responding although picture content had to be ignored. This interference with responding can be interpreted as reflecting automatic (i.e., unintentional) affective associations with the target stimuli (cf. ${ }^{129}$ ). Clearly, this pattern of results of the sex-AST casts doubt on the role of automatic global affective 
associations in vaginismus. The current results with the sex-AST are in line with earlier findings indicating that ambivalent emotions and negative affect in response to erotic stimuli do not impede automatic responses revealing genital arousal 46,67,68,130. Together, the current findings suggest that only if women with vaginismus have sufficient opportunity to utilize additional information and have sufficient resources for more deliberate information processing, more negative appraisals of sex stimuli may arise. Thus on the basis of the present findings deliberate rather than automatic affective appraisals of vaginal penetration seem the most logical target for clinical interventions.

The considerable improvement of deliberate global affective appraisals of sex following exposure treatment was not expected, since basic research has demonstrated that affective valence is typically insensitive to extinction procedures $79,116,131$. However, it should be noted that the current exposure therapy was not restricted to extinction procedures but also contained various components that might be conceptualized as counterconditioning interventions. The latter might have been responsible for the treatment-induced change of the self-reported global affective associations with sex. For example, during treatment, the women learn to control and relax their pelvic floor muscles, gradually inserting their finger(s) in their vagina, to replace the feeling of lack of control (representation of the UCS) by the ability to control the pelvic floor muscles (incompatible representation). Although self-reported global affect clearly improved following treatment, the increase in positive affect did not reach the range of positive affect scores of the symptom-free women. Thus, there is still ample room for further improvement.

Previous research has shown that not only residual subjective fear scores ${ }^{109}$, but also residual (negative) affective associations 81 are predictors of a return of fear and avoidance behavior. Therefore, it seems important to seek for alternative means to further improve the impact of therapeutic interventions on deliberate global affective associations with vaginal penetration. Strengthening the global positive affective associations with vaginal penetration might be the gateway towards improving sexual pleasure. 


\section{Limitations}

The sexual stimuli were presented under laboratory conditions which might have impaired sexual readiness. Therefore, it remains to be seen whether similar results will occur in real life contexts and in conditions of heightened sexual arousal 132,133. In addition, the vaginal penetration pictures that were used in this study might not fully represent the stimuli that women with vaginismus actually fear. A challenge for future research is to develop more ideographic stimuli which may help to increase the external validity of laboratory research.

The present findings do not allow any firm conclusions about the causal properties of the relatively negative deliberate associations with sexual stimuli in the development or persistence of symptoms in women with vaginismus. Although the positive changes in deliberate appraisals of sexual stimuli combined with the diminished complaints of vaginismus after exposure treatment are consistent with the view that global affect plays a role in the maintenance of symptoms, it would require more specific experimental manipulation of global affect to arrive at more solid conclusions in this respect.

\section{Conclusions}

The current findings suggest that more deliberate threat and more global affective appraisals are both involved in vaginismus. We could not provide support for the hypothesized role of more automatic appraisals in the generation and maintenance of vaginismus. Taken together, the pattern of findings indicates that subjective threat and global affect are the most promising targets for clinical interventions. Although the current exposure treatment was already quite effective in reducing deliberate fear and improving global affect, there is still considerable room for improvement as selfreported evaluations of sexual stimuli following exposure treatment remained more negative than those of symptom-free women. Improvement of the efficacy of interventions on global affect with sexual stimuli might create opportunities to improve sexual functioning following exposure treatment. 



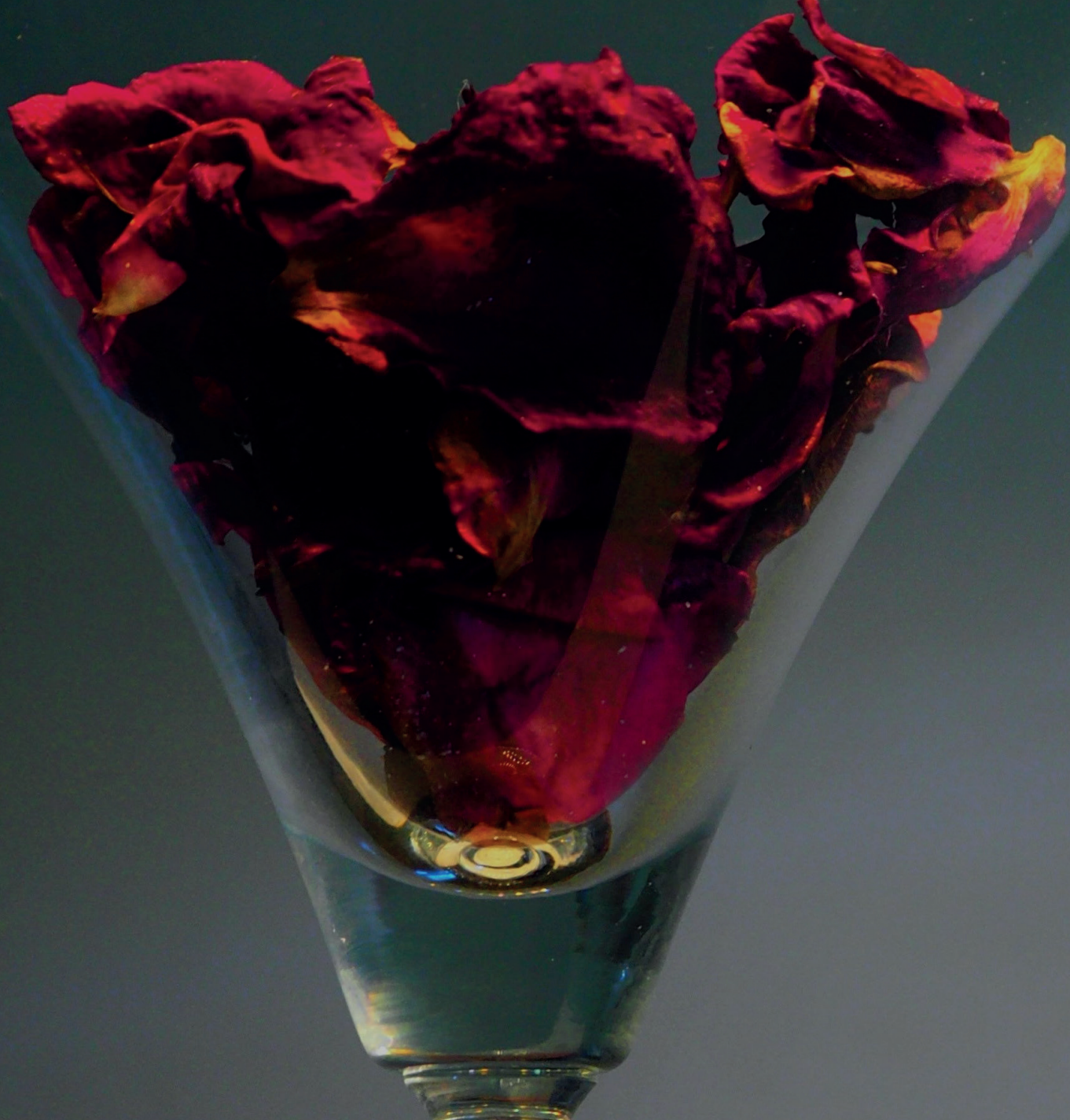

\section{Chapter 4}

Therapist-Aided Exposure for Women with Lifelong Vaginismus:

Mediators of Treatment Outcome: A Randomized Waiting List Control Trial

M.M. ter Kuile, R.J. Melles, C.C. Tuijnman-Raasveld, H.E. de Groot \& J.J. van Lankveld Journal of Sexual Medicine, 2015, 12, 1807-1819 


\section{ABSTRACT}

Introduction: Therapist-aided exposure seems an effective treatment for lifelong vaginismus, but mechanisms of action have not yet been established.

Aims: The purpose of the present study was to investigate whether treatment outcome of a therapist-aided exposure treatment was mediated by changes in positive and negative penetration beliefs or feelings of sexual disgust.

Methods: Participants with lifelong vaginismus were allocated at random to a 3-month exposure $(n=35)$ or a waiting-list control condition $(n=35)$.

Main outcome measures: Full intercourse was assessed daily during 12 weeks. Secondary outcome measures (complaints about vaginismus and coital pain) were assessed at baseline and after 12 weeks. Possible mediators: penetration beliefs (catastrophic-pain beliefs; genital incompatibility beliefs; perceived control beliefs) and feelings of sexual disgust were assessed at baseline and 6 weeks.

Results: Treatment outcome (coital frequency, symptoms of vaginismus and coital pain) at 12 weeks was mediated by changes in negative and positive penetration beliefs at 6 weeks, in particular by more pronounced reduction of catastrophic-pain penetration beliefs. No evidence was found that changes in feelings of sexual disgust mediated treatment outcome.

Conclusions: The results strongly suggest that therapist-aided exposure affects negative penetration beliefs and that these changes in negative penetration beliefs mediate treatment outcome in women with lifelong vaginismus. Implications for treatment are discussed. 


\section{INTRODUCTION}

Vaginismus is defined in DSM IV-TR as an involuntary contraction of the musculature of the outer third of the vagina interfering with intercourse, causing distress and interpersonal difficulty ${ }^{10}$. Together with dyspareunia, vaginismus has been merged into the new DSM-5 diagnosis called 'Genito-pelvic pain/penetration disorder' ${ }^{9}$. The present study focused on women with lifelong vaginismus, who have never experienced complete vaginal intercourse.

Epidemiological studies often subsume vaginismus in more generalized questions about pain with intercourse resulting in few accurate prevalence estimates ${ }^{134}$. The best estimate of reported rates varies between $0.4-6.0 \%$ 135-138. In more sexually conservative cultures, there appears to be a significantly higher rate of vaginismus ${ }^{139-}$ 142. Vaginismus has been reported as the most important reason for seeking help for sexual problems in Turkey. In Western and Southern Europe, 14\% - 25\% of women who attend sexual dysfunction clinics for sexual problems describe vaginismus as their primary concern ${ }^{143,144}$.

The etiological determinants of lifelong vaginismus have as yet not been definitively identified ${ }^{11}$. Conservative and religious attitudes, lack of sex education, sexual abuse, and relationship factors have all been reported as potential causal risk variables; however, none have been confirmed empirically ${ }^{11}$. Vaginismus is classified as a sexual dysfunction; however, little research information is available on the sexual function and response of women with lifelong vaginismus. While some women and their partners report few sexual problems if vaginal penetration is not anticipated or attempted, others find their sexual functioning significantly compromised ${ }^{11}$.

Based on the fear-avoidance model of Vlaeyen and Linton ${ }^{34}$, a circular fear-avoidance model for vaginismus has been proposed 2,145. The basic tenet of the model is that catastrophic thinking about vaginal penetration will give rise to vaginal penetrationrelated fears. Increased fear facilitates increased pelvic muscle tone, resulting in pain or failed attempts. Some components of this model received empirical support. For example cross-sectional studies found that women with lifelong vaginismus show a specific pelvic muscle defense reflex in response to potential vaginal penetration 48 , 
display significantly more defensive reactions and report more anxiety compared to women without vaginismus during pelvic examination ${ }^{13}$. Moreover, they reported higher levels of negative penetration beliefs 35,53,146 and coital fear 59,109.

Vaginismus, as conceptualized in this model may be akin to a specific phobia. Exposure to the feared stimulus is essential in overcoming a phobic disorder, and the disconfirmation of dysfunctional cognitions about the feared object, which is enabled by being exposed to it, is considered germane for the resolution of the phobic fear. To test this hypothesis, a therapist-aided exposure treatment was developed ${ }^{58}$. In a recent randomized controlled trial (RCT) that included 70 women with lifelong vaginismus, therapist-aided exposure was found successful in enabling women to experience full intercourse in $89 \%$ of treated couples, compared to $12 \%$ in untreated couples ${ }^{59}$. Based on the fear-avoidance model, we aim here to investigate whether treatment outcome of a therapist-aided exposure therapy for lifelong vaginismus is mediated by a reduction in negative penetration beliefs.

In addition to negative penetration beliefs, there are some indications that more positive appraisals such as perceived control beliefs are also important in the treatment of women with sexual pain problems. Women with dyspareunia who reported increased perceived pain control following cognitive-behavioral therapy (CBT) also reported less coital pain ${ }^{147}$. This finding suggests that positive penetration beliefs may also constitute important psychological mechanisms of change in the treatment of women with vaginismus.

Furthermore, it has been suggested 128 that sexual disgust may also play an important role in the causation and maintenance of vaginismus. Disgust is a defensive mechanism serving to protect the body from contamination with pathogens. Sexual disgust may also trigger defensive responses to anticipation of penetration, e.g., defensive contraction of pelvic floor muscles ${ }^{128}$. In support of this assumption, levator-nasi muscle activity, as a unique physiological expression of disgust, was found specifically enhanced in women with vaginismus, when they were exposed to a women-friendly sex video clip 40.

In sum, although there is evidence that therapist-aided exposure is an effective in the treatment of lifelong vaginismus, no effort has been devoted to empirically testing 
whether changes in negative or positive penetrations beliefs and feelings of sexual disgust, which are assumed to mediate treatment effectiveness, actually constitute mechanisms of change. We investigated (1) whether in comparison with a waiting list control condition, exposure affected penetration beliefs and feelings of sexual disgust at 6 weeks, (2) whether changes in penetration beliefs or feelings of sexual disgust at 6 weeks were related to changes in outcome at 12 weeks and (3) whether treatment outcome at 12 weeks was mediated by these changes in the mediation measures.

\section{MATERIALS AND METHODS}

\section{Design}

The study design comprised a between-group comparison of an active treatment and a waiting-list control condition (WLC). Both conditions included assessments at baseline, 6, and 12 weeks. After the 12-week assessment, waiting-list participants started with the active treatment. Participants were treated in two research centers in the Netherlands. Randomization was block-stratified by site to ensure equal properties in each treatment arm. Two research assistants who were not involved in treatment delivery performed assessment at each research center. Approval for the study was obtained from the medical ethical committees of both hospitals involved (P06.130), oral and written informed consent was obtained from all the participants.

\section{Participants}

Our study sample consisted of 70 patients with lifelong vaginismus, who were selected for participation in an outcome study of the efficacy of therapist-aided exposure for women with lifelong vaginismus (for more details see ${ }^{59}$ ). Twenty-four (34\%) patients were recruited from a sexology outpatient clinic of two university hospitals and 46 (66\%) participants responded to advertisements in local and national newspapers. To 
be included in the outcome study, patients were required to meet the following criteria: heterosexual woman, aged 18 years or older, with a diagnosis of lifelong vaginismus, living in a relationship of at least three months' duration and in good general health as evidenced by medical history. The diagnosis of lifelong vaginismus was assessed after taking a full sexual history. The lifelong nature of the complaint was judged by the assessor (master student in clinical psychology), based on the applicant's self-report. Participants were excluded if they: had had full sexual intercourse at any time; received a somatic explanation for the complaints of vaginismus (using standardized physical examination); had been given a DSM IV-TR diagnosis of affective disorder, psychotic disorder, substance- related disorder or posttraumatic stress disorder related to the genitals (e.g., as a sequel to sexual abuse) using a standardized psychiatric interview; or did not speak Dutch well enough to participate.

The mean age of the 70 participants was $28.91(+7.34)$ years and $54(77 \%)$ were living together with a partner. The mean duration of the relationship was $6.18(+5.63)$ years. Four participants had children and had become pregnant by self-insemination with the semen of their own partners, but still suffered from vaginismus. The mean duration of the complaint was $10.41(+6.54)$ years. Fifty-three participants $(76 \%)$ had previously undergone unsuccessful treatment for the complaint.

\section{Procedure}

Applicants for the study contacted the institute by telephone or mail. The first screening occurred by telephone and if the participants fulfilled the main inclusion criteria, written information was sent by mail. After one week, the research assistant contacted the applicant to ask if she wanted to participate. A pretreatment assessment with both partners was scheduled at the hospital. After general instructions on the study procedure were given and the informed consent was obtained, the sexual history was taken and a standardized psychiatric interview conducted to check exclusion criteria. After completion of the self-report questionnaires, all participants underwent a standardized physical examination ${ }^{25}$ by a doctor, who was not involved in treatment delivery. The goals of this exam were (a) exclusion of physical causes of vaginismus, (b) 
reassurance of participants who were concerned about physical causes of their condition and (c) to investigate vulvar pain. Couples were then randomly allocated, to either active treatment or WLC, per treatment center.

\section{Treatment and Therapists}

Therapist-aided exposure consisted of a total of three 2-hr sessions at the hospital, during which the participant was exposed to the feared penetration objects, i.e. dilators, fingers and tampons. During a therapist-aided exposure session, the female therapist and the partner were in the room. Exposure during the hospital session was selfcontrolled, as the patient self-performed vaginal penetration exercises. These exercises at the hospital were followed up with the same exercises at home. After an introductory session in the first week, three therapist-aided exposure sessions were scheduled in the second week, followed by two follow-up sessions over a period of 5 weeks, in which homework was discussed and questions were answered. In all cases, the partner accompanied the participant during the full treatment session. The treatment procedure in the present study was similar to the procedure used in our previous study, described in detail elsewhere ${ }^{59}$.

Four female psychologists and a female social worker with two years of training in sexology conducted the treatment. The four psychologists involved in treatment delivery each had at least 15 years of experience in treating women with vaginismus. Weekly supervision sessions were held at each center. A group supervision session was held once a month with all the therapists to ensure competence and adherence to the protocol. Special permission for the psychologists to be therapists for this trial was obtained from the Medical Ethical Committees of the Hospitals.

\section{Main outcome measures}

Frequency of sexual intercourse was assessed using a Diary. The participants answered the following question every day for a period of 12 weeks: 'Have you had sexual 
intercourse with your partner, including full penile penetration of the vagina?' Possible answers were on a 3-point scale: (a) 'I have not attempted to have sexual intercourse' (coded 0), (b) 'I have attempted to have intercourse, and have not had full penile penetration into my vagina' (coded 0), and (c) 'I have attempted to have intercourse, and have had full penile penetration into my vagina' (coded 1). Based on these daily dichotomized categories (coded 0 and 1) a weekly 'frequency of sexual intercourse score' was derived for the period between 6 and 12 weeks after the baseline ${ }^{58}$. To investigate symptoms of vaginismus, the 4-item subscale for 'vaginismus' of the Golombok Rust Inventory of Sexual Satisfaction (GRISS) (scoring ranges: 4-20) was used 148-150. For example a GRISS-vaginismus item is 'Do you find that your vagina is so tight that your partner's penis cannot enter it?' and is answered on 5-point scale from never to always. Higher scores indicate higher problem severity. Cronbach's alpha in the current sample was .94. To investigate coital pain, the 3-item subscale for 'sexual pain' of the Female Sexual Function Index (FSFI) (scoring ranges: 0-6) was used 97,98. For example, an FSFI coital pain item is 'Over the past 4 weeks, how would you rate your level (degree) of discomfort or pain?' and is answered on a 6-point scale from '0 = Did not attempt intercourse, $1=$ Very high to $5=$ Very low or none at all during or following vaginal penetration?" A higher score indicates less sexual pain. Cronbach's alpha in the current sample was .99.

\section{Putative mediator measures}

To assess negative and positive beliefs regarding vaginal penetration, the 5-item 'Catastrophic-pain beliefs", the 2-item 'Genital incompatibility beliefs' and the 4-item 'Control beliefs' scales of the Vaginal Penetration Cognition Questionnaire (VPCQ) were used ${ }^{35}$. The VPCQ has positive and negative statements about vaginal penetration, and is answered on a 7-point Likert scale. For example a VPCQ catastrophic-pain belief item is 'I am afraid I cannot do anything to change the pain from penetration'. Higher scores indicate a higher level of the specific penetration belief measured. Cronbach's alpha of the VPCQ scales for 'Catastrophic-pain beliefs', 'Genital incompatibility beliefs' and 'Perceived control beliefs' in the current sample were respectively .92, .91 and .90. Feelings of sexual disgust were assessed using the 3 -item sexual disgust subscale of a 
familiar person (SDQ-D-Familiar) of the 48-item Sexual Disgust Questionnaire (SDQ) ${ }^{151}$. Items are answered on a 9-point Likert scale. This subscale of the SDQ assessed the degree of disgust an individual would experience when experiencing contact with certain sexual stimuli stemming from a familiar person, this being one's self or the partner (SDQ-D-familiar) respectively. For example a sexual disgust item is 'Use a towel for your face after it has been used to wipe off sperm from your partner after sexual intercourse and smells'. Higher scores on this subscale indicated higher levels of disgust. Cronbach's alpha of the SDQ-subscale in the current sample was .83.

All measures were validated for Dutch populations 35,58,97,98,149-151.

\section{Statistical analyses}

Following the three-step process for assessing mediation effects as described by Baron and Kenny (1986) 152, first we investigated whether treatment (exposure vs. WLC) affected the putative mediators (negative and positive penetration beliefs or feelings of sexual disgust) (a-path) at 6 weeks and outcome (frequency of sexual intercourse, symptoms of vaginismus and coital pain) (c-path) at 12 weeks. Therefore, we performed Group (exposure, WLC) x Time (baseline, 6 weeks (or 12 weeks)) repeated-measures ANOVAs with the putative mediators or outcome measures as within-subject factors. To control for possible differences between the two research centers, treatment location was added as a fixed factor in all the ANOVAs. In addition, we calculated within-groups Cohen's d and between-groups Cohen's d (exposure vs. WLC group) to obtain an estimate of the magnitude of the changes resp. differences ${ }^{125}$.

Secondly, we investigated whether changes in the putative mediator variables (penetration beliefs/sexual disgust) at 6 weeks were related to outcome at 12 weeks (bpath). We therefore computed standardized residualized gain scores (RGS) and conducted Pearson product-moment correlation coefficients. This was done by regressing the scores at 6 weeks on the putative mediating variables or the outcome scores at 12 weeks on the respective baseline scores for all study participants. The standardized residual for each subject is the residual divided by the sample standard 
deviation of the residuals. The standardized residuals have a mean of 0 and a standard deviation of 1 .

Thirdly, we examined the role of a putative mediator on the association of treatment with outcome, in which simple mediation models were used. The association between treatment and outcome has to disappear/shrink (c') when the process measure is also added to the model ${ }^{152}$.

More specifically, we investigated 1) the association of treatment with outcome at 12 weeks, controlling for baseline outcome levels; 2) the association of treatment with a putative mediator at 6 weeks, controlling for baseline putative mediator levels; 3) the association of the putative mediator at 6 weeks with outcome at 12 weeks controlling for baseline levels on the outcome and putative mediator measures; and 4) the attenuation of the association of treatment with outcome after accounting for changes in mediator from baseline to 6 weeks. This way, we could analyze whether treatment predicted changes in the putative mediator at 6 weeks and whether these changes predicted changes in outcome at 12 weeks. The significance of the indirect effect of treatment on outcome through changes in the putative mediator was determined using a bootstrap approximation with 1000 iterations to obtain biased-controlled confidence intervals. In order to get an impression of the strength of the indirect effect, we calculated the ratio of $\beta$ of the indirect effect to $\beta$ of the total effect. When the indirect effects of treatment were significant through more than one mediator, we conducted a multiple mediation model, including the significant mediators of the simple mediation analyses. We could thus analyze the independent effect of each of the mediators over and above the effect of the other mediators.

Analyses were run using SPSS v. 22 (IBM Corporation, Armonk, NY) and MPlus v. 7. Missing data in SPSS were handled using Multiple Imputation Chained Equations (MICE) 153 and in Mplus analyses using Full Information Maximum Likelihood (FIML) estimation for missing data ${ }^{154}$. A significance level of $\mathrm{p}<.05$ was used for all analyses. 


\section{RESULTS}

\section{Preliminary results}

In total, seven of the 70 participants (10\%) left the study before the assessment at 12 weeks. Three of the four couples that dropped out in the treatment condition did so because the relationship ended (one before treatment and two after treatment) (see Figure 1). T-tests for independent samples revealed no differences between dropouts and participants who completed treatment $(\mathrm{p}<.05)$ on demographic or pretreatment levels on the putative mediator or outcome variables.

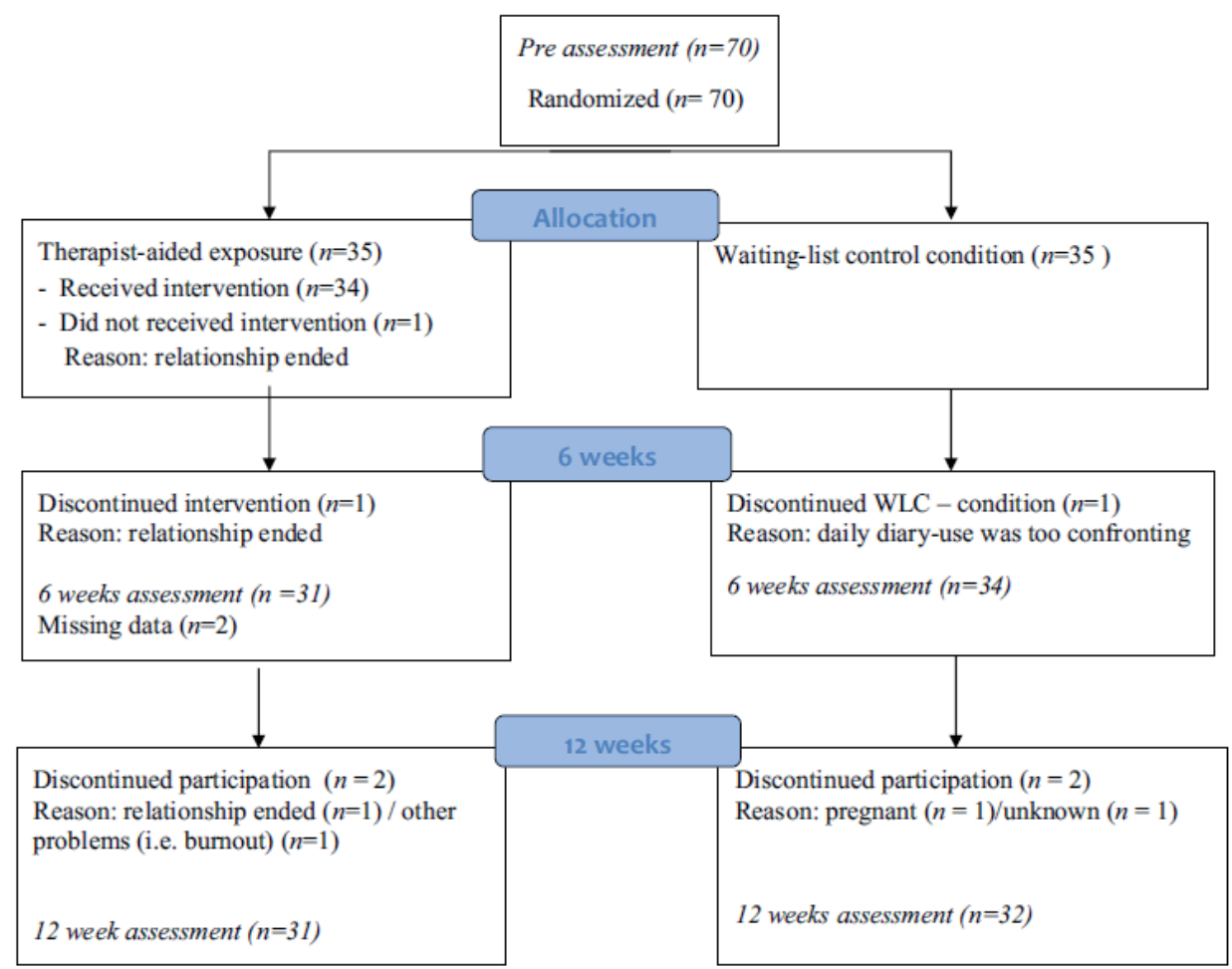

Figure 1 Flow of participants through each stage of the study.

WLC = waiting list condition 


\section{Effect of treatment on the outcome measures at 12 weeks}

As also reported in our main outcome study ${ }^{59}$, the coital frequency was significantly increased $(\mathrm{d}=1.36)$ and symptoms of vaginismus $(\mathrm{d}=1.95)$ and coital pain $(\mathrm{d}=1.74)$ were significantly reduced in the treatment condition compared to the WLC at 12 weeks (see Table 1).

\section{Effect of treatment on the proposed mediators at 6 weeks}

As depicted in Table 1, catastrophic-pain beliefs and genital incompatibility beliefs were significantly reduced and control beliefs during vaginal penetration were significantly increased in the treatment condition compared to WLC at 6 weeks. The differences found in these putative mediators between treatment and WLC at 6 weeks can be considered as large $(0.88<\mathrm{d}<1.76)$. Feelings of sexual disgust by a familiar person were not affected by treatment. 


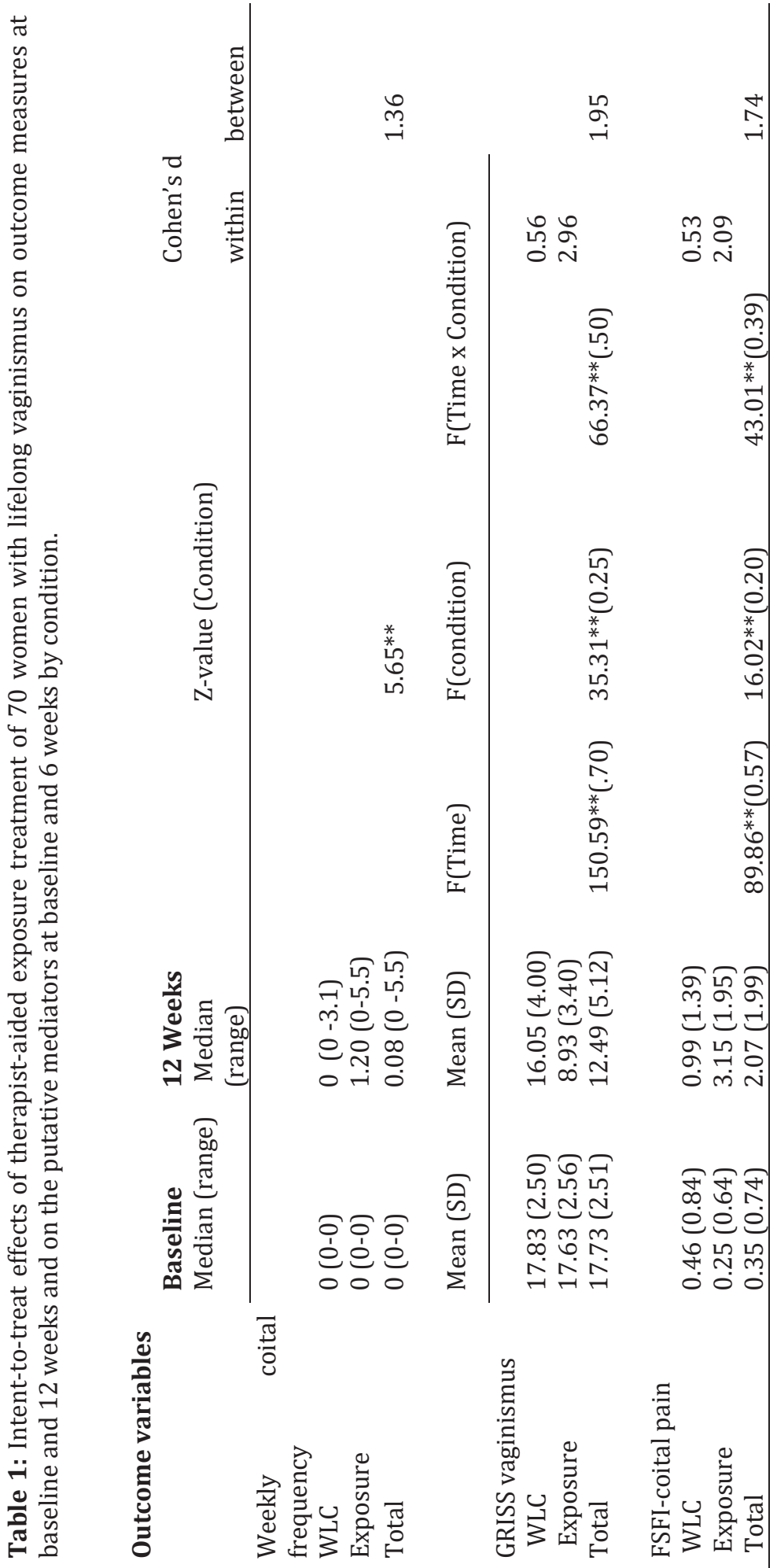




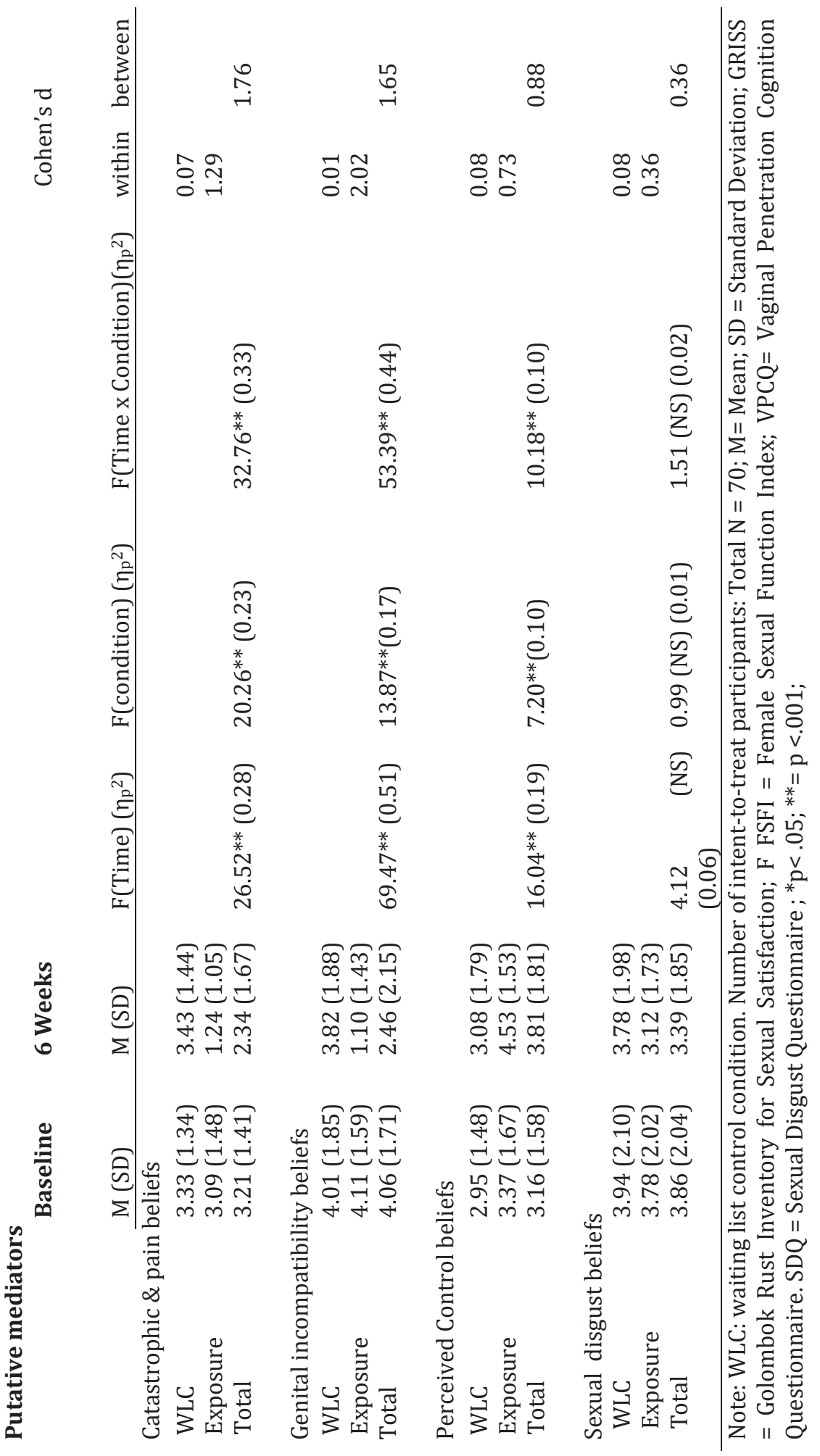


Table 2: Inter correlations between the changes in the putative mediators at 6 weeks and the changes between the putative mediators at 6 weeks with changes in outcome at 12 weeks in the total group of 70 women with lifelong vaginismus.

RGS 6 weeks

\begin{tabular}{|c|c|c|c|c|c|}
\hline & & \\
\hline & & $\begin{array}{l}\text { VPCQ } \\
\text { catastrophic } \\
\text { pain }\end{array}$ & $\begin{array}{l}\text { VPCQ } \\
\text { Incompatibly }\end{array}$ & $\begin{array}{l}\text { VPCQ } \\
\text { Perceived } \\
\text { control }\end{array}$ & $\begin{array}{l}\text { SDQ } \\
\text { Sexual } \\
\text { disgust }\end{array}$ \\
\hline \multirow{3}{*}{ RGS 6 weeks } & Putative mediators & & & & \\
\hline & VPCQ Catastrophic pain & & $0.73 * *$ & $-0.75^{* *}$ & 0.20 \\
\hline & VPCQ Incompatibility & & & $-0.42^{* *}$ & $0.29 *$ \\
\hline \multirow{5}{*}{ RGS 12 weeks } & VPCQ Perceived Control & & & & -0.04 \\
\hline & Outcome & & & & \\
\hline & Frequency of coitus & $-0.60^{* *}$ & $-0.48^{* *}$ & $0.45^{* *}$ & -0.13 \\
\hline & GRISS vaginismus & $0.69^{* *}$ & $0.61^{* *}$ & $-0.44^{* *}$ & 0.18 \\
\hline & FSFI coital pain & $-0.60 * *$ & $-0.52 * *$ & $0.40^{* *}$ & -0.09 \\
\hline
\end{tabular}

Note: ${ }^{*} \mathrm{p}<.05 ;{ }^{* *}=\mathrm{p}<.001$

Number of intent-to-treat participants: Total $\mathrm{N}=70$; RGS= Residual Gain Score; GRISS = Golombok Rust Inventory for Sexual Satisfaction; FSFI = Female Sexual Function Index; VPCQ= Vaginal Penetration Cognition Questionnaire; SDQ = Sexual Disgust Questionnaire

\section{Association between changes in the putative mediators at 6 weeks and outcome at 12 weeks}

As can be seen in Table 2, changes in catastrophic-pain beliefs, genital incompatibility beliefs, perceived-control beliefs at 6 weeks were significantly related in the expected direction with frequency of coitus, and changes in symptoms of vaginismus and coital pain at 12 weeks. The effect sizes of these significant correlations varied between $|.40|<$ $r<|.69|$ and can be considered as medium to large (see Table 3). No significant associations were found between changes in sexual disgust at 6 weeks and changes in outcome measurements at 12 weeks. 
Table 3: Bootstrapping estimates for the simple and multiple mediation models of the indirect relationship between treatment (exposure vs. waiting list control ) on outcome at 12 weeks through the putative mediators at 6 weeks in 70 women with lifelong vaginismus.

\begin{tabular}{|c|c|c|c|c|c|c|}
\hline \multirow[b]{3}{*}{$\begin{array}{l}\text { Outcome/Putative } \\
\text { mediator }\end{array}$} & \multicolumn{6}{|c|}{ Bootstrapping 1000 samples } \\
\hline & \multicolumn{3}{|c|}{$95 \% \mathrm{CI}$} & \multicolumn{3}{|l|}{$95 \% \mathrm{CI}$} \\
\hline & $\begin{array}{l}\text { Estimate A x } \\
\text { B }\end{array}$ & Lower & Upper & Estimate C' & Lower & Upper \\
\hline \multicolumn{7}{|l|}{ Coitus frequency } \\
\hline Catastrophic - pain ${ }^{\mathrm{a}}$ & 0.290 & 0.115 & 0.465 & 0.227 & -0.064 & 0.519 \\
\hline Perceived control ${ }^{\mathrm{b}}$ & 0.095 & 0.029 & 0.161 & 0.415 & 0.220 & 0.609 \\
\hline Genital incompatibility & 0.107 & -0.042 & 0.256 & 0.427 & 0.180 & 0.673 \\
\hline Sexual Disgust & -0.010 & -0.045 & 0.024 & 0.543 & 0.378 & 0.708 \\
\hline Totalab & 0.287 & 0.105 & 0.469 & 0.236 & -0.059 & 0.530 \\
\hline \multicolumn{7}{|l|}{ Symptoms of Vaginismus } \\
\hline Catastrophic - pain a & -0.285 & -0.448 & -0.122 & -0.435 & -0.665 & -0.204 \\
\hline Perceived controlb & -0.083 & -0.159 & -0.006 & -0.631 & -0.789 & -0.472 \\
\hline Genital incompatibilityc & -0.157 & -0.329 & -0.013 & -0.571 & -0.795 & -0.348 \\
\hline Sexual Disgust & 0.006 & -0.025 & 0.037 & -0.724 & -0.848 & -0.600 \\
\hline Totalabc & -0.329 & -0.519 & -0.139 & -0.398 & -0.642 & -0.155 \\
\hline \multicolumn{7}{|l|}{ Coital Pain } \\
\hline Catastrophic - pain ${ }^{\mathrm{a}}$ & -0.263 & -0.461 & -0.064 & -0.322 & -0.629 & -0.016 \\
\hline Perceived control & -0.083 & -0.167 & 0.001 & -0.498 & -0.703 & -0.292 \\
\hline Genital incompatibility & -0.088 & -0.210 & 0.034 & -0.506 & -0.706 & -0.307 \\
\hline Sexual disgust & 0.006 & -0.033 & 0.045 & -0.598 & -0.767 & -0.430 \\
\hline
\end{tabular}

Note: Estimate A x B = indirect effect; Estimate C': direct effect (treatment on outcome); $\mathrm{CI}=$ confidence interval.

\section{Mediation of the effect of treatment on outcome}

Below, we will discuss the mediation process for each of the outcome measures separately.

As can be derived from Table 3, of the simple mediation models of coital frequency on treatment, only the bootstrapping estimates of the mediation effects of catastrophic-pain beliefs $(ß=.29 ; 95 \% \mathrm{BCI}=.12-.47)$ and perceived control beliefs $(\beta=.10 ; 95 \% \mathrm{BCI}=$ $.03-16$ ) were significant, excluding zero in the 95\% confidence interval. For the simple mediation model with catastrophic-pain beliefs as mediator, the bootstrapping estimate 
for the direct effect of treatment on coital frequency c' (.22) was no longer statistically significant, indicating that most of the treatment effect on coital frequency was mediated by changes in catastrophic-pain beliefs during treatment.

In order to get an impression of the strengths of the indirect effect, we calculated the ratio of $\beta$ of the indirect effects. For catastrophic-pain beliefs, the ratio $\beta(.29)$ to $\beta$ of the total effect $(.52)=.56$. For perceived-control beliefs, the ratio $\beta(.10)$ to $ß$ of the total effect (.52) =.19. Finally, both mediators were entered in a multiple mediation model. The total bootstrapping estimate for the indirect effect of both mediators together was significant ( $\beta=.29$ ). Only the specific indirect effect through catastrophic-pain beliefs was significantly different from zero in this multiple mediation model (see Figure 2). In the multiple mediation model, the direct effect of treatment on coital frequency was no longer statistically significant. The strength of the indirect effect of this multiple model was .40 (.29/.52) and very comparable with the strength of the indirect effect of .40 in the simple mediation model with catastrophic-pain beliefs as sole mediator.

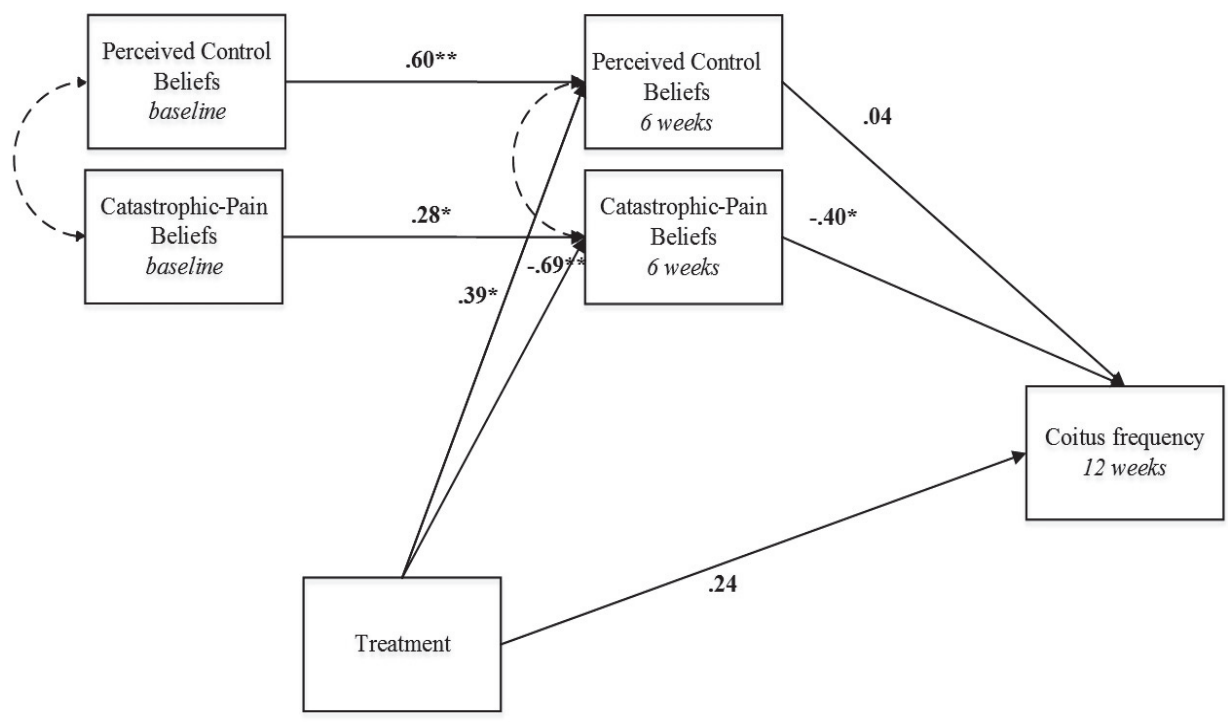

Figure 2: ${ }^{*} \mathrm{p}<.05 ;{ }^{* *} \mathrm{p}<.001$. Parameter estimates for the multiple mediation model of the indirect relationship between treatment (exposure vs. waiting list control ) and outcome at 12 weeks (coitus frequency) through two mediators at 6 weeks (penetration beliefs) in 70 women with lifelong vaginismus. Solid line - single-headed arrow path coefficients represent standardized partial regression coefficients. Dashed line - two-headed arrows represent correlation coefficients. 
Bootstrapping estimates of the indirect relationship between treatment and symptoms of vaginismus showed that the simple mediation effects of catastrophic-pain beliefs $(ß=$ $-.29 ; 95 \% \mathrm{BCI}=-.45--.12)$, genital incompatibility beliefs $(ß=-.16 ; 95 \% \mathrm{BCI}=-.33-$ $.01)$ and perceived control beliefs $(ß=-.08 ; 95 \%$ BCI $=-.16-.01)$ were all significant. The strength of the indirect effects for catastrophic-pain beliefs was: .40 (.29/.72), for genital incompatibility beliefs: $.22(.16 / .73)$ and for perceived control beliefs: .11(.08/.71). Finally, the three mediators were entered in a multiple mediation model and the total indirect effect of the three mediators was significant $(ß=-.33 ; 95 \% \mathrm{BCI}=-.52--.14)$. The strength of the indirect effect of the three mediators together was .45 (.33/.73) and very comparable with the strength of the indirect effect (.40) in the simple mediation model with catastrophic-pain beliefs as sole mediator. Only the specific indirect effect through catastrophic-pain beliefs was significantly different from zero in this multiple mediation model (see Figure 3).

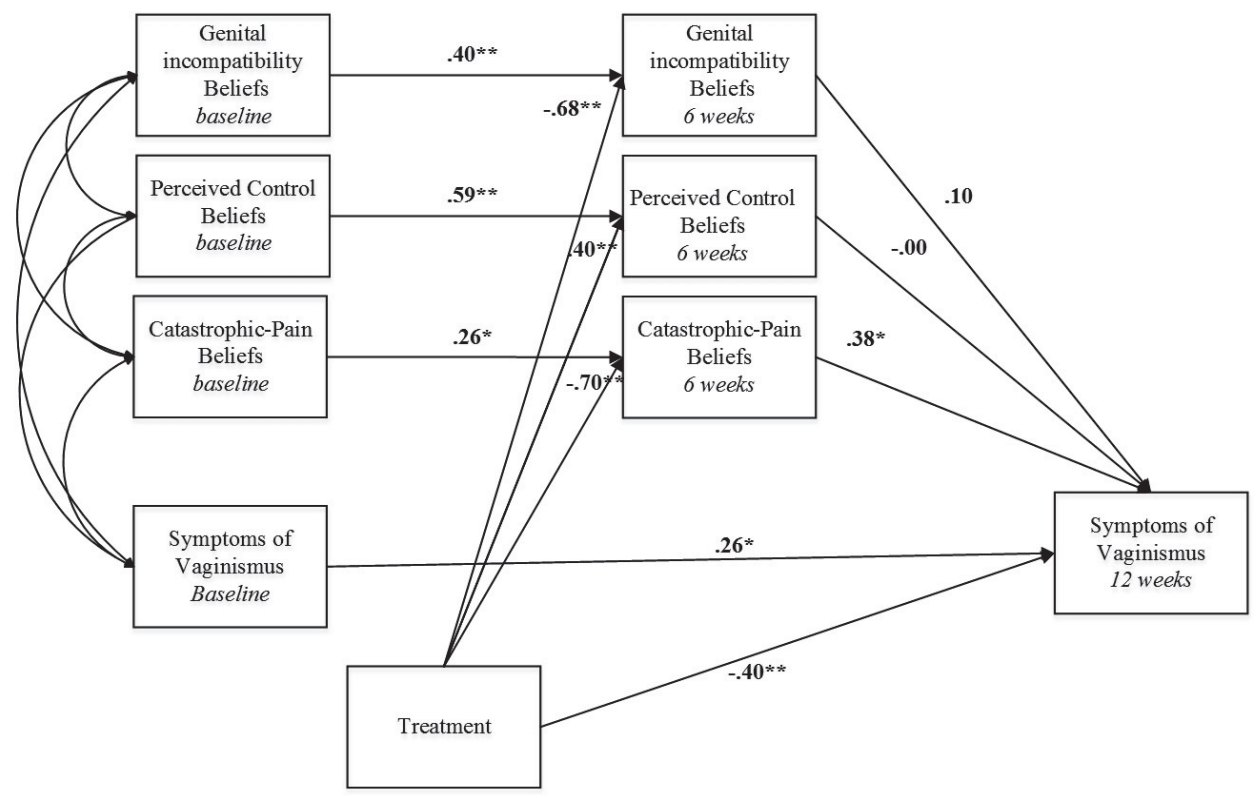

Figure $3:^{*} \mathrm{p}<.05 ;{ }^{* *} \mathrm{p}<.001$. Parameter estimates for the multiple mediation model of the indirect relationship between treatment (exposure vs. waiting list control) and outcome at 12 weeks (symptoms of vaginismus) through three mediators at 6 weeks (penetration beliefs) in 70 women with lifelong vaginismus. Solid line - single-headed arrow path coefficients represent standardized partial regression coefficients. Dashed line - two-headed arrows represent correlation coefficients 
Bootstrapping estimates of the indirect relationship between treatment and coital pain showed that only the simple mediation effect of catastrophic-pain beliefs ( $\beta=-.26$; $95 \%$ $\mathrm{BCI}=-.46--.06)$ was significant. The strength of the indirect effect for catastrophic-pain beliefs was: .44 (.26/.58) (see Figure 4).

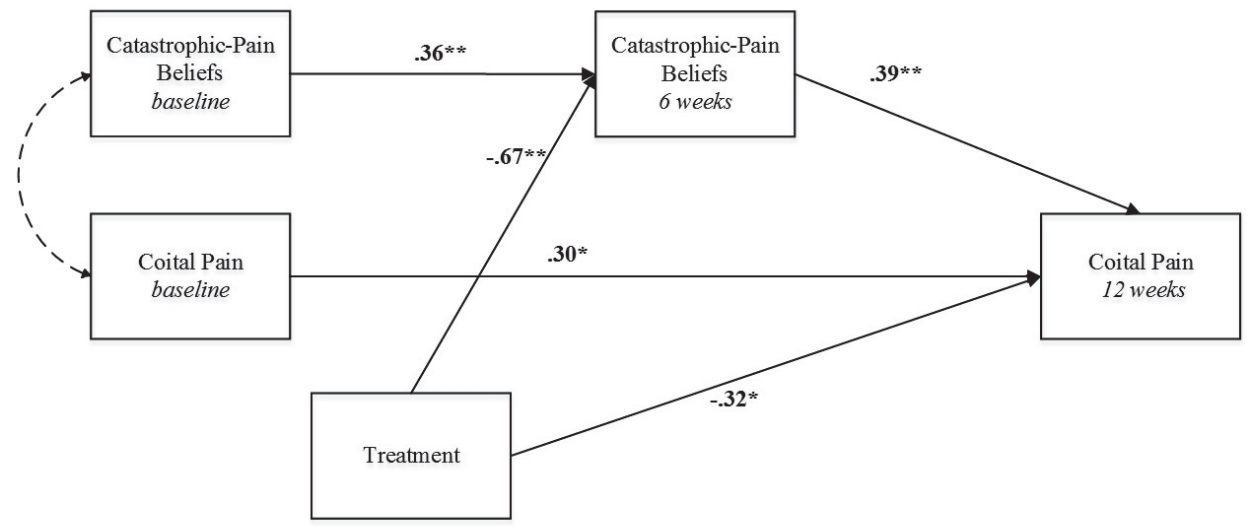

Figure 4: ${ }^{*} \mathrm{p}<.05 ;{ }^{* *} \mathrm{p}<.001$. Parameter estimates for the simple mediation model of the indirect relationship between treatment (exposure vs. waiting list control) and outcome at 12 weeks (coital pain) through the mediator at 6 weeks (penetration beliefs) in 70 women with lifelong vaginismus. Solid line single-headed arrow path coefficients represent standardized partial regression coefficients. Dashed line two-headed arrows represent correlation coefficients

\section{DISCUSSION}

Our research question was whether treatment outcome of a therapist-aided exposure treatment would be mediated by positive and negative penetration beliefs or feelings of sexual disgust. We found that treatment outcome at 12 weeks was mediated by changes in negative and positive penetration beliefs at 6 weeks, in particular by more pronounced reduction of catastrophic-pain penetration beliefs. No evidence was found that changes in feelings of sexual disgust mediated treatment success. 
The results indicate that catastrophic-pain penetration beliefs and genital incompatibility beliefs were significantly reduced after 6 weeks of exposure treatment compared to a waiting list control condition. Treatment outcome measures (coital frequency, symptoms of vaginismus, and coital pain) at 12 weeks were mediated by changes in negative penetration beliefs at 6 weeks, in particular by the more pronounced changes in catastrophic-pain penetration beliefs. It is striking that a reduction in catastrophic-pain beliefs was a significant mediator for all of the three outcome measures studied: symptoms of vaginismus, coital pain and frequency of coitus. Therapist-aided exposure was found to increase perceived-control penetration beliefs and these changes at 6 weeks were related to higher coitus frequency and a reduction in symptoms of vaginismus at 12 weeks. The finding that higher levels of perceived control in women with lifelong vaginismus was associated with better outcome is in line with the findings in women with dyspareunia 147. However, changes in perceived control beliefs and changes in catastrophic-pain beliefs were highly correlated $(r=-.75)$. Based on the current findings, we could not consider the unique indirect effect of changes in perceived-control penetration beliefs above and beyond the more pronounced changes in catastrophic penetration beliefs.

On the basis of our findings, we may conclude that a reduction in catastrophic-pain penetration beliefs is the strongest mediator of treatment success in the treatment of lifelong vaginismus. It is worth mentioning that the multiple mediator models of the three outcome measures studied: symptoms of vaginismus, coital pain and frequency of coitus, which also included the other putative mediators in the model, did not improve the percentage of explained variance above and beyond the variance explained by a simple mediation model with catastrophic pain beliefs as sole mediator. However, we have to keep in mind that the reduction in catastrophic pain beliefs was highly associated with a reduction in genital incompatibility beliefs and in perceived control beliefs $(.42<\mathrm{r}<.75)$. This common phenomenon of multicollinearity, or redundancy among mediators, may also explain the finding, although regardless of the total indirect effect in multiple mediation models being significant, that in two of the three multiple mediation models none of the individual putative mediators showed a unique significant indirect effect 155 . 
In the current study we did not find any indication that sexual disgust mediated treatment outcome in women with lifelong vaginismus. So there are no indications that feelings of sexual disgust, as assessed with the SDQ, are directly related to changes in sexual penetration related measures. It should be acknowledged, however, that the SDQ examines disgust toward sexual by-products (e.g., sperm) and does not directly refer to sexual penetration or sexual organs. Therefore whether women with vaginismus would also report heightened disgust toward sexual intercourse and/or their partner's penis 151 still remains to be established, and if that is so, whether that would mediate any treatment outcome.

Do the current findings support the fear-avoidance model of vaginismus? According to the fear-avoidance model of vaginismus, the negative penetration beliefs are maintained because avoidance prevents disconfirmation of the catastrophic penetration beliefs. By directly reducing avoidance and increasing successful penetration behaviors, negative penetration beliefs are disconfirmed. When catastrophizing is reduced and eventually eliminated, avoidance behavior as a coping strategy is no longer necessary. As we found that the large reduction of negative penetration beliefs and avoidance behavior only occurred as a consequence of the exposure treatment, we may conclude that exposure treatment disconfirmed the negative penetration beliefs. The fear-avoidance model, however, specifies that the changes in negative penetration beliefs have to occur before changes in outcome occur. As both penetration beliefs and outcome variables changed significantly during the first six weeks of treatment ${ }^{109}$ and we only assessed penetration behavior on a daily basis in contrast to the mediator variables, the current study is unable to answer matters relating to the temporal order of changes, i.e. whether changes in negative penetration beliefs predicted subsequent changes in outcome, or whether changes in negative penetration beliefs represent an epiphenomenon of symptom change ${ }^{156}$. More definitive conclusions about the mechanism of change await further study, in which daily measures of mediator variables and outcome variables are warranted.

What do these findings indicate for clinical practice? The study results strongly suggest that therapist-aided exposure affects negatives penetration beliefs and these changes in negative penetration beliefs mediate treatment outcome in women with lifelong 
vaginismus. Exposure gives the opportunity to test these beliefs and to examine to what extent the feared consequences actually occur. We think that in the treatment of lifelong vaginismus, therapist-aided exposure has a number of advantages over using homebased exposure only. For example, the initial exposure session takes place in a professional setting with a knowledgeable therapist, increasing the women's feelings of reassurance. The role of the therapist is to guide the woman (and the partner) through the difficulty of approaching the penetration-related fears, to manage the associated intense fear, and encourage non-avoidance. If the participant (and her partner) has clear catastrophic cognitions about what could (or could not) occur during the penetration exercises, the therapist helps the participant (and partner) to verbalize these expectations. In these instances, the exposure exercises are used as behavioral experiments. Furthermore, the therapist can actively assist the couple in learning how best to practice exposure at home and support the couple in overcoming difficulties as they occur. Exposure treatment carried out at home is a possible alternative, but the role of the therapist is crucial in focusing the couple on confronting avoidance and managing anticipatory catastrophic thoughts. It should be acknowledged, however, that we had only modest success in our first randomized control trial on CBT for lifelong vaginismus 57 , in which only $18 \%$ of the participants reported intercourse after CBT combined with home-based exposure only compared with $89 \%$ after therapist-aided exposure in the present study. Also in our former study, it was found that outcome was partly mediated by changes in fear of coitus and avoidance behavior, however penetration beliefs were not assessed in that study ${ }^{109}$. Based on the findings of our two RCTs, we hypothesize that therapist-aided exposure is more effective in changing penetration beliefs than CBT with home-based exposure. However, further studies are warranted to investigate this hypothesis within one single study design.

The primary limitation of the present study was that we could not demonstrate the temporal precedence of changes in the proposed mediators predicting subsequent changes in outcome as discussed above. Furthermore, in the current study genital pain was assessed with the FSFI, as the FSFI is not designed to measure pain, it may be limited in this respect. However as half of the participants avoid genital penetration situations, it was not possible to use a more specific measure to assess pain in the current population of women with lifelong vaginismus, such as the genital pain measure 
157. Nevertheless, with respect to the participants who could have intercourse and completed the FSFI subscale and a more specific genital pain measure 157 , we found that both measures were highly correlated $(\mathrm{r}=.73)$, so we may conclude that the findings of the present study are not critically dependent on the measure we used. Further research is necessary to develop a genital pain measure that can be used in all patients with Genito-pelvic pain/penetration disorder.

Some strengths of the current study also deserve attention. This is the first randomized control trial directly comparing the effect of exposure treatment on putative mediating processes in the treatment of lifelong vaginismus. In addition, repeated assessments of outcome and mediating variables allowed prospective mediation analyses.

Although statistical prediction models obtained in a specific sample inherently include the risk of representing a fit only with the data in the studied sample, and thus call for replication in different samples, we conclude that the current study results strongly suggest that changes in penetration beliefs mediate treatment outcome in women with lifelong vaginismus. 



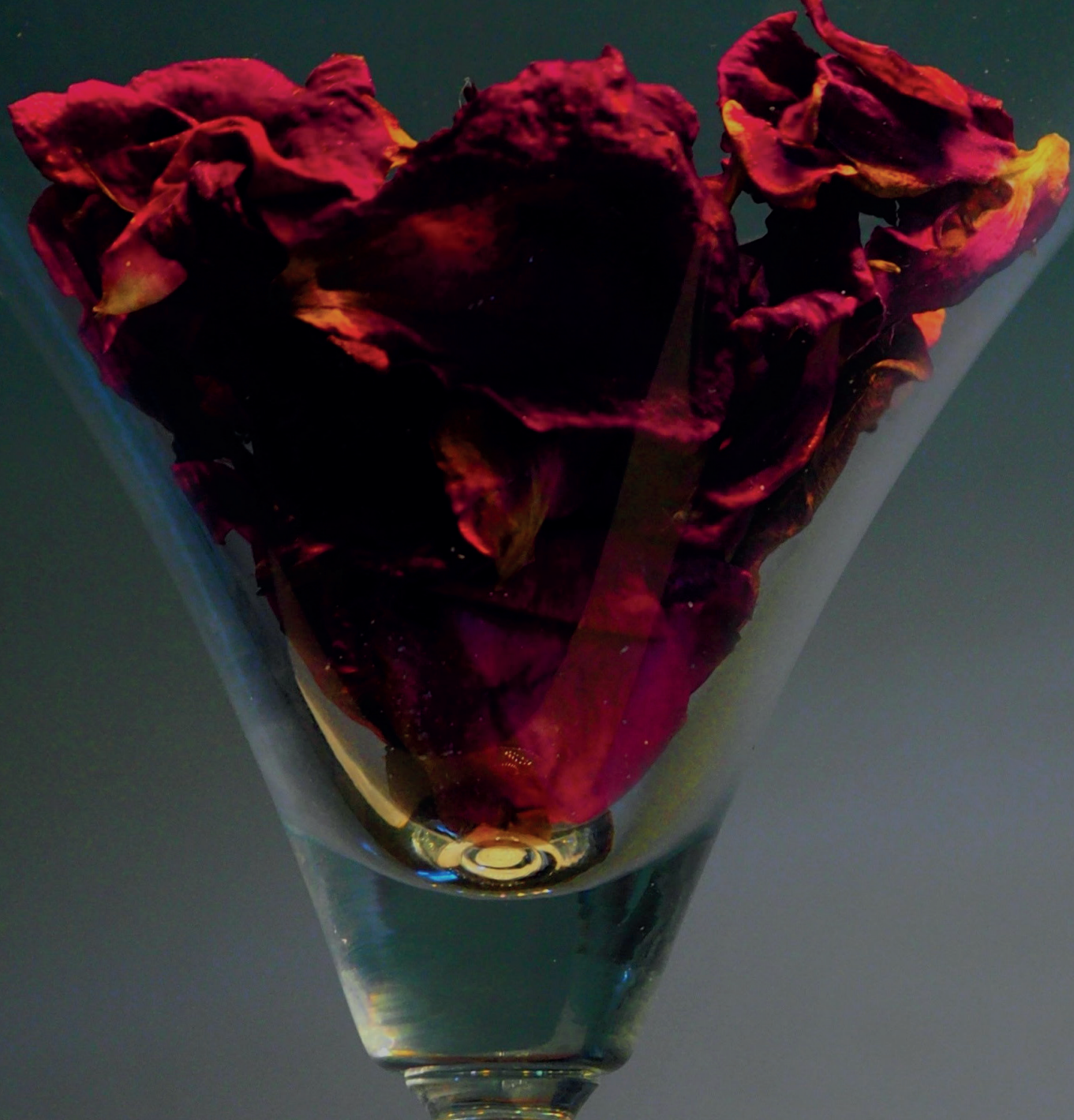

\section{Chapter 5}

The Vaginal Pressure Inducer:

A new device to test the (un)pleasurableness and tolerance of vaginal pressure and the influence of sexual stimuli

R.J. Melles, M.D. Dewitte, M.M. Ter Kuile, C. Bonnemayer, M.L. Peters Journal of Sex \& Marital Therapy, 2017, 44, 189-200 


\section{ABSTRACT}

To better understand the impact of sexual stimuli on genital pain, a new instrument was developed: the Vaginal Pressure Inducer (VPI). We administered gradually increasing vaginal pressure with the VPI to sexually functional women while watching a neutral, erotic or explicit sex film. Women had higher unpleasantness thresholds in a sexual context compared to a non-sexual context. Moreover, ratings of pleasurableness were higher in the sexual compared to neutral context and most so during the explicit sexual film. These results provide initial support for the suitability of the VPI to study determinants of pleasant and unpleasant appraisal of vaginal pressure.

\section{INTRODUCTION}

The influence of sexual arousal on genital pain (sensitivity) is unclear. Genital pain is most often elicited by tactile stimulation of the vulva-vaginal region and most frequently located at the vaginal introitus. This highly sensitive genital area is involved in both positive sexual states such as arousal and orgasm, - leading to vasocongestion ${ }^{158}$ of the vestibular bulbs and lubrication 159- and negative sensations such as genital pain. Impaired subjective sexual arousal, lack of lubrication and increased pelvic floor activity are assumed to be risk factors for genital pain 2,33,160. However, studies found contradictory results concerning sexual arousal in women with genital pain. Compared to sexual functioning women, women with genital pain showed impaired 47,66 as well as unaffected ${ }^{46}$ levels of genital sexual arousal and impaired 46 as well as unaffected 47,66 subjective levels of sexual arousal in response to sexual film. Activity of the pelvic floor muscles has been shown to be activated by negative emotions, i.e. threat and fear (of pain) 23,24,61, as well as positive emotions like sexual arousal 62-65,161. Taken together, these findings suggest that genital sensitivity, vaginal pressure, sexual arousal and activity of the pelvic floor muscles are interrelated and associated with emotional states. 
Several theoretical models ascribe an important role to subjective as well as genital sexual arousal during vaginal penetration to prevent and overcome intercourse pain 2,33 . Linking this with the aforementioned findings on the interrelation between sexual arousal, genital sensitivity/pressure and pelvic floor activity, it is surprising that so far only three studies assessed the influence of sexual stimuli on genital (pain) sensitivity. One study determined vaginal sensitivity, while the two other studies focused on sensitivity of the vulvar vestibule. These studies show contradicting results on changes in genital sensitivity and vaginal pressure tolerance as a function of sexual arousal.

In the study of Gruenwald 76 probes were inserted into the vagina to assess vaginal vibratory sensation 162 in response to sexual film clips. Results revealed that touch sensitivity of the vagina did not significantly change as a function of sexual arousal ${ }^{76}$. In two other studies, a vulvagesiometer ${ }^{163}$ was used to induce punctuate pressure on the vulvar vestibule to measure pain thresholds ${ }^{54,77}$ and pleasurable sensitivity ${ }^{77}$. Sexual arousal was measured by self-report to almost having an orgasm by masturbation ${ }^{77}$, and by rating subjective levels of sexual arousal as well as by use of the labial thermistor clip to measure physiological sexual arousal in response to sexual film clips ${ }^{54}$. Both studies found that pain sensitivity of the vulvar vestibule increased during sexual arousal 54,77. Pleasurable sensitivity did not significantly change as a function of high subjective sexual arousal 77. Both studies also measured touch thresholds of the vulvar vestibule as a function of sexual arousal using graded disposable filaments. Whereas sexual arousal induced by sexual films clips increased touch sensitivity ${ }^{54}$, masturbation did not affect touch sensitivity of the vulvar vestibule ${ }^{77}$.

In sum, the evidence so far seems to indicate that sexual arousal increases genital pain sensitivity 54,77 and does not affect pleasurable sensitivity ${ }^{77}$, whereas the results for genital touch sensitivity are contradictory with either no change 76,77 or increased sensitivity ${ }^{54}$ as a function of sexual arousal. These findings contradict the important role of sexual arousal to prevent or overcome sexual pain/penetration pain 2,78.

One way to explain these unexpected findings is that the punctual pressure for sensory testing might not be an accurate reflection of the pressure during masturbation and partner sex, which is a more pronounced and prolonged experience. The possibility of applying vaginal pressure in an - at least partially - pleasurable way would provide the 
opportunity to test if pleasurable genital touching together with subjective arousal can influence the subjective perception (pleasantness) of vaginal pressure along with an increase of the tolerance of vaginal pressure. To further assess the influence of sexual arousal on the subjective experience of vaginal pressure, a more prolonged, pronounced and dispersed vaginal pressure in a sexual context is required.

To our knowledge, only one study induced a more prolonged and dispersed vaginal pressure in the vaginal introitus using an inflatable soft rubber balloon, to test painful sensation in women with and without genital pain ${ }^{164}$. Although this instrument seems well suited to induce vaginal pressure and asses prolonged and dispersed pressure in the vaginal introitus to assess vulvar (pain) sensitivity, a disadvantage is that a well inflated balloon tends to slide out of the vagina. To induce (higher rates of) vaginal pressure an instrument is needed to keep the inflated balloon in the vaginal introitus, which may also improve the standardization of the induced vaginal pressure. For this purpose, we have developed a new instrument: the Vaginal Pressure Inducer (VPI, see Figure 1). To assess the subjective experience of vaginal pressure, we explicitly asked participants to rate the subjective experience of pleasurable as well as painful pressure on the vagina in order to prevent priming the participants to appraise any physical sensation as painful rather than pleasurable. Also note that pleasurable sensitivity is typically a more important sexual motivator than (avoidance of) pain ${ }^{77}$.

The aim of the current study was to test the suitability of this new device, the VPI, to measure the subjective perception and tolerance of vaginal pressure in sexually functioning women. We expected that prolonged and gradually increasing vaginal pressure in a context of sexual arousal will increase the pleasurableness and the unpleasantness threshold of vaginal pressure. We also explored whether the level of sexual arousal matters as well, with high levels of sexual arousal leading to larger increases in pleasurable sensitivity/ decreases in pain sensitivity compared to low levels of sexual arousal. Additionally, we explored the influence of vaginal pressure on subjective sexual arousal during sexual films. 


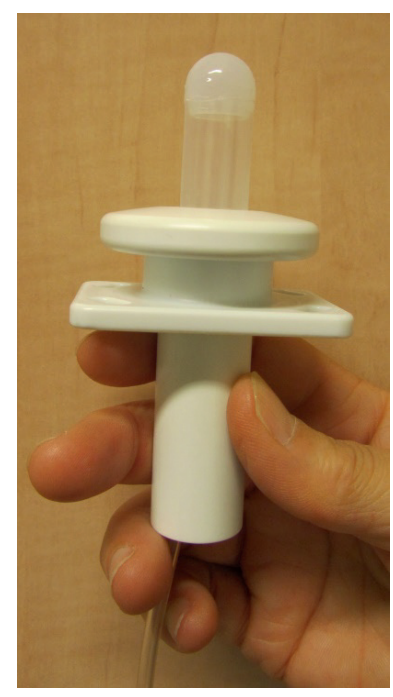

Figure 1: Vaginal Pressure Inducer (VPI)

\section{METHODS}

\section{Participants}

Forty-two women were included in the study. The sample was recruited through advertisements at the university, in the local media, and by directly asking people. Inclusion criteria included absence of sexual problems, aged between 18 and 45 years, good command of the Dutch language, a steady heterosexual relationship for at least 3 months and being sexually active including coitus. Women were excluded in case of pregnancy, breast-feeding, (post-)menopause, major affective disorder, psychotic disorder, substance related disorder, posttraumatic stress disorder resulting from abuse in the area of the pelvic floor and the genitals (e.g., sexual assault) according to DSM-IVTR criteria ${ }^{10}$ or if they were taking medication that was likely to interfere with sexuality. The study was approved by the Ethical Committee. 


\section{Materials}

\section{Stimulus materials}

We selected six film excerpts of 7 minutes each. These six films consisted of one neutral acclimatization film, two high arousal (HA) sexual films, one low arousal (LA) sexual film, a HA non-sexual film and a neutral film. Both HA sexual film excerpts depicted manual and oral sexual and intercourse scenes. The LA sexual film excerpt depicted heterosexual seducing scenes from the same films with dressed actors showing petting and kissing. Bare body parts, genital touching or fragments of behavior to undress were omitted from the LA sexual film. To control for effects of high arousal per se, we also presented a HA non-sexual film, comprising high emotion eliciting excerpts from the films the Eight Day, Life is beautiful and Forrest Gump, ranked with highest scores on positive affect and (non-sexual) arousal 165.

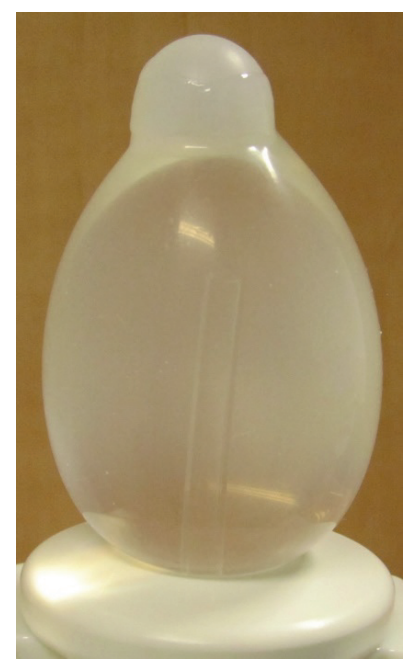

Figure 2: VPI with max filled balloon 


\section{Description of the Vaginal Pressure Inducer (VPI)}

The VPI consists of an inflatable vaginal balloon that is partially inserted into a synthetic handle (Figure 1). The handle ends in a flange, which can be placed against the opening of the vagina. The flange prevents too deep insertion, to ensure introital pressure. In the middle of the flange a balloon is fixed by pumping just enough water (initial value), so that the balloon extends 2 centimeters out of the flange. The balloon is gradually filled with body temperature water by a pump until a length of $4-6 \mathrm{~cm}$. When the balloon is filled an outward omnidirectional pressure is given to the surrounding tissues. The tube of the balloon is connected to a pump to fill the balloon gradually and controllably with water ( $0.83 \mathrm{ml}$ per second). The maximal volume of pumped water after 120 seconds is $100 \mathrm{ml}$, by which the inflatable balloon can reach a maximal circumference of $16 \mathrm{~cm}$ outside the body (Figure 2).

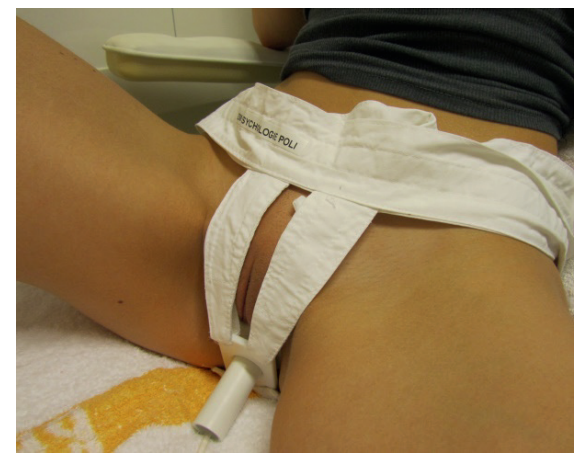

Figure 3: VPI with straps

To insert and fixate the VPI an extension piece is placed around the handle with four openings to attach velcro straps, which are subsequently attached to a waist band to create a kind of pants. By wearing these pants and- tailoring the straps the flange is naturally placed against the vulva inserting the balloon into the introitus of the vagina (Figure 3). Figure 4 shows the inflated balloon after 20 seconds; for the picture the velcro straps are removed. The VPI can be put on by the participant herself and can be 
remotely controlled to respect privacy of the participant. Because the level of pressure on the vaginal wall can be influenced by physical variances and fluctuations of the pelvic floor muscles activity, a pressure gauge is used to allow an objective measure of induced pressure on the vaginal wall. To control temperature, one thermometer is fixed at the underside of the balloon inside the handle. By use of isolated tubes and a hot water tray with thermometer the water is kept at circa $37^{\circ} \mathrm{C}$. A new sterilized balloon is used for each woman. The VPI is sterilized after each experimental session by use of alcohol.

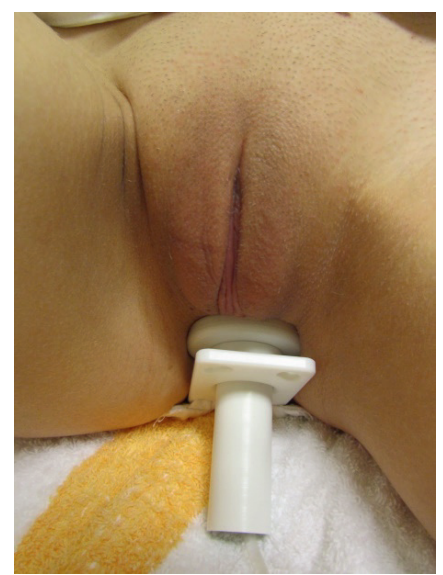

Figure 4: VPI inserted balloon straps removed

\section{PROCEDURE}

The experiment consisted of a single session that took place in a sound-attenuated laboratory room at the university. After obtaining informed consent the participant was led to the test room where she received detailed information about the set-up of the experiment. The participant was instructed to stop the vaginal pressure as soon as the pressure felt unpleasant by use of a button. Then the participant was left alone to prepare herself using audio support from the research assistant if needed. When the participant was ready to start, the computer program started. To acclimatize the participant, the experiment started with the presentation of a neutral acclimatization 
film with pressure induction. Because the data of the acclimatization trial are not critical for the aims of the study, these data are not included in the present paper. The experiment continued with one of the HA sexual films without vaginal pressure, followed by 4 randomized conditions with pressure: a HA sexual film, a LA sexual film, a HA non-sexual film and a neutral film. Which of the two HA sexual films was paired with the VPI was counterbalanced between participants (see Figure 5).

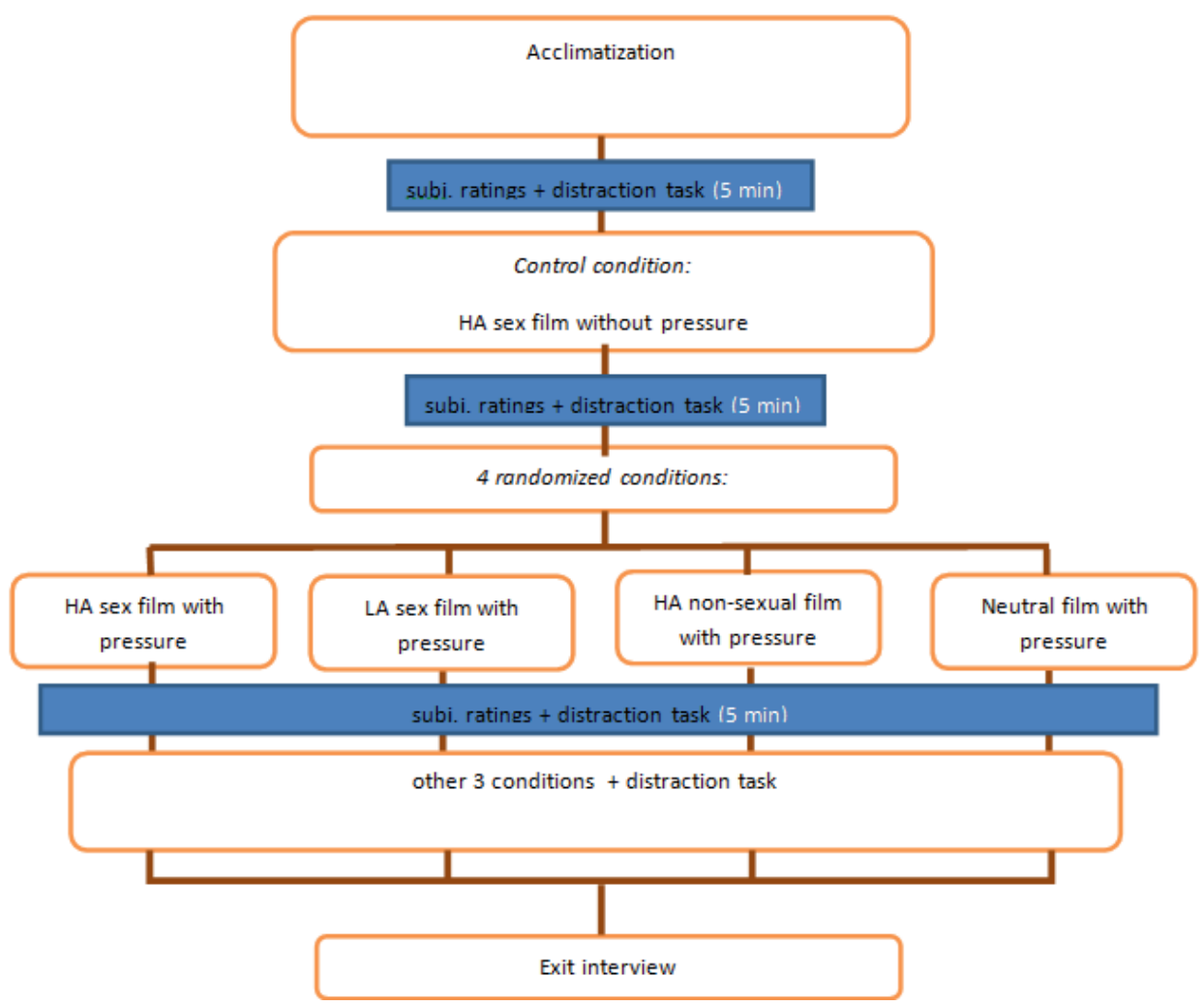

Each condition started with short written information about that condition and a reminder to check the fastening of the instrument (VPI). In all conditions with pressure induction, two minutes after the start of the film the vaginal balloon was gradually filled until the participant pressed a button in order to terminate the pressure. By pressing the 
button the movie stopped and the VPI balloon was immediately deflated to its initial level by draining the water. If the participant did not press the button, the movie ended after 7 min. (i.e. 5 min. of vaginal pressure). To prevent carry-over effects, in between films women completed a letter span working memory task of 5 minutes. Then the next film excerpt was presented with the same procedure, until all conditions were administered.

In order to control the instruments the level of vaginal pressure and temperature ratings were shown by a feedback bar on the monitor of the researcher. At the end of the experiment, a short exit interview took place to probe women's reactions to the experimental procedure followed by a questionnaire regarding the film clips. The total duration of the experimental session was about $1 \frac{1}{2}$ hour.

\section{Main outcome measures}

\section{Dependent measures}

Threshold of Unpleasant Vaginal Pressure in seconds (TVPs)

The time taken to reach the Threshold of Unpleasant Vaginal Pressure (TvP) from start pressure to stop pressure/ film is reported in seconds (TVPs). When the button was not pressed, the TvP was coded as 300 seconds (end of the film clip).

\section{Subjective ratings}

After each condition the participant was asked to rate the subjective experience of pleasurable and painful vaginal pressure sensations ("Watching the film to which level did you experience the following feeling? ("pleasurable/ painful pressure on the vagina") and the subjective sexual arousal ("At this moment, to which level do you feel sexually aroused?" on three seven-point Likert scales with the extremes on a 7 point 
scale "not at all" to "very strong" (range:1-7). Higher scores indicates higher levels of pleasureable/ painful pressure sensations and subjective sexual arousal.

\section{Statistical analyses}

To calculate the influence of the four conditions (HA sexual, LA sexual, HA nonsexual, neutral) on the output measures (subjective sexual arousal, unpleasantness thresholds and the ratings of pleasurable and painful vaginal pressure) we conducted repeated measure ANOVA with condition as a within-subjects factor. If the Mauchly's test of sphericity indicated that the assumption of sphericity had been violated for the 4 conditions, we corrected degrees of freedom using Greenhouse-Geisser estimates of sphericity $(\varepsilon=.33)$. Contrasts were used to compare the conditions to each other.

\section{RESULTS}

\section{Feasibility and tolerability}

The VPI was well tolerated by women. None of the women showed negative reactions or interrupted the experiment. The women valued the experiment on a 10-point Likert scale ranging from "unpleasant" to "pleasant" with a mean score of 7.2 (range 1-10). Exit interviews revealed mainly positive reactions of women concerning the experiment varying from "a bit weird", to "stimulating", "interestingly"; with respect to the vaginal pressure, reactions ranged from "not very pleasant" to "doable". 


\section{Descriptive data}

Fifty five women were interested in participation in the current study. 11 women were excluded due to: no relationship (8), use of antidepressants (2) and no response (1). After inclusion 2 women dropped out due to illness, leading to a sample of 42 women. One experimental session was terminated prematurely due to sound problems. Due to technical problems output data of the unpleasantness thresholds of two women were not saved. Consequently, subjective ratings were obtained from 41 women, complete datasets (including the thresholds and intravaginal pressure) were obtained from 39 women.

The mean age of the women was 24 year (range 17-35, SD 6 years) and the women were in general well educated (54\% higher education). 38 women used hormonal contraception. The mean score of the Female Sexual Functioning Index 97,98 was 30.4 (range 19-35; $\mathrm{SD}=3.4$ ), indicating that sexual functioning was within a normal range 95 .

Mean mmhg (millimeter of mercury) values across conditions varied between 111.29 mmhg at start of the film clip (initial value vaginal pressure) to $279.35 \mathrm{mmhg}$ when the pressure was terminated, with a maximum measured value of $473.35 \mathrm{mmhg}$. Mean, minimum and maximum values of the unpleasantness thresholds (in seconds), the intravaginal pressure at threshold time (in $\mathrm{mmhg}$ ) and the subjective ratings of pleasurable and painful pressure and sexual arousal across conditions are shown in Table 1. 
Table 1: Unpleasantness thresholds, intravaginal pressure and subjective ratings by Condition

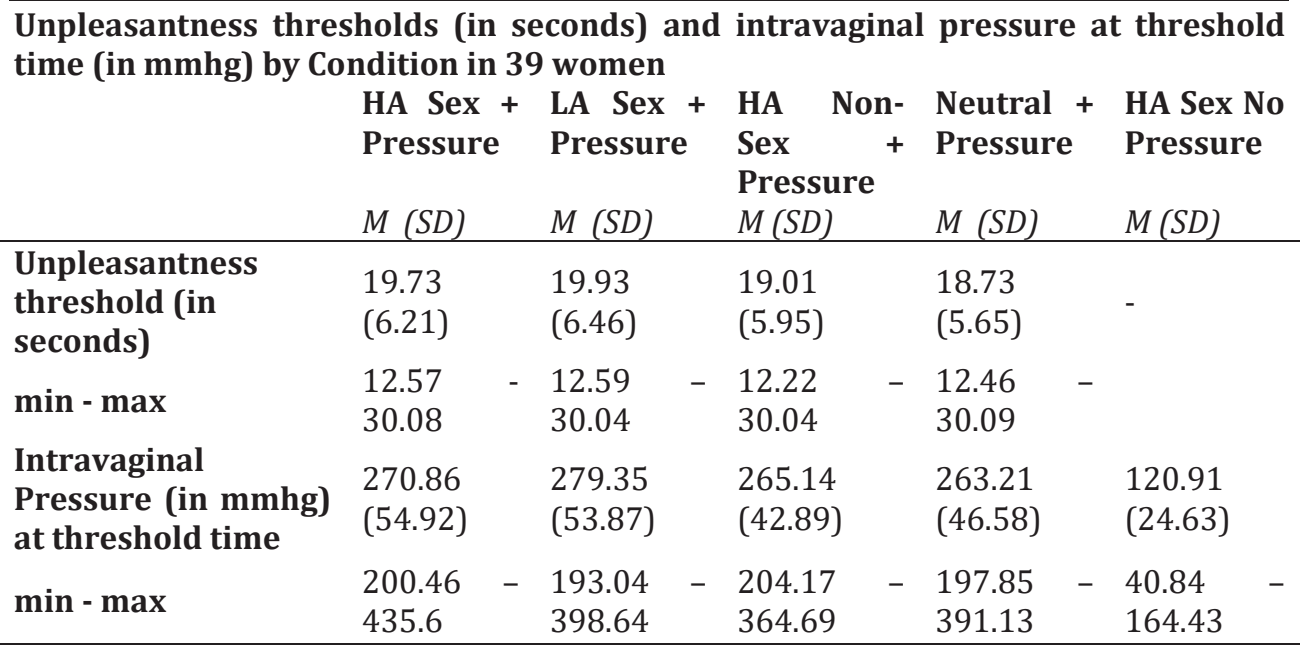

Pleasurable and Painful Pressure \& Sexual Arousal by Condition: Mean scores (7point scale) in 41 women

\begin{tabular}{|c|c|c|c|c|c|}
\hline & $\begin{array}{l}\text { HA Sex + } \\
\text { Pressure }\end{array}$ & $\begin{array}{l}\text { LA Sex + } \\
\text { Pressure }\end{array}$ & $\begin{array}{lr}\text { HA Non- } \\
\text { Sex } \quad+ \\
\text { Pressure }\end{array}$ & $\begin{array}{l}\text { Neutral + } \\
\text { Pressure }\end{array}$ & $\begin{array}{l}\text { HA Sex No } \\
\text { Pressure }\end{array}$ \\
\hline $\begin{array}{l}\text { Pleas } \\
\text { Press }\end{array}$ & & $3.44(1.6)$ & $2.33(1.62)$ & $2.81(1.6)$ & $3.02(1.72)$ \\
\hline Painful Pressure & $\begin{array}{l}2.49 \\
(1.49)\end{array}$ & $2.61(1.6)$ & $2.81(1.6)$ & $2.81(1.54)$ & $1.15(.42)$ \\
\hline $\begin{array}{l}\text { Subjective Sexual } \\
\text { Arousal }\end{array}$ & $\begin{array}{l}4.15 \\
(1.51)\end{array}$ & $2.73(1.4)$ & $1.81(1.1)$ & $1.98(1.35)$ & $3.49(1.44)$ \\
\hline \multicolumn{6}{|c|}{$\begin{array}{l}\text { Note: HA Sex + Pressure = High Arousal Sex film with Pressure; LA Sex + Pressure = } \\
\text { Low arousal Sex film with Pressure; HA Non-Sex + Pressure = High Arousal Non- } \\
\text { Sexual film with Pressure;; HA Sex No Pressure = High Arousal Sex film without } \\
\text { Pressure; } \boldsymbol{M}=\text { Mean rates from start pressure to stop film (pressure). SD = Standard } \\
\text { Deviation }\end{array}$} \\
\hline
\end{tabular}

\section{Manipulation check sexual stimuli}

There was a significant main effect of condition on sexual arousal $F(2.5,100.07)=56.11$, $\mathrm{p}<.001, \mathrm{r}=.36$. Contrasts revealed that ratings of sexual arousal of the HA sexual film 
$F(1,40)=84.18, p<.001, r=.68$ and the LA sexual film $F(1,40)=16.29, p<.001, r=.29$ were significant higher than the ratings of the neutral film; the ratings of sexual arousal of the HA sexual film were significant higher than the ratings of the LA sexual film $F(1,40)=41,05, p<.001, r=.5$.

\section{Unpleasantness Threshold}

There was a significant main effect of condition on the threshold of unpleasant vaginal pressure (TVPs) $\mathrm{F}(2.38,90.51)=4.76, \mathrm{p}<.01, \mathrm{r}=.05$. Contrasts revealed that compared to the neutral film, both the HA sex film $F(1,38)=4.95, p<.05, r=.12$. and the LA sex film $\mathrm{F}(1,38)=7.91, \mathrm{p}<.01, \mathrm{r}=.17$ increased the threshold of unpleasant vaginal pressure. When compared to the HA nonsex film, the threshold of unpleasant vaginal pressure was also increased by both sex films: the HA sex film $F(1,38)=5.07, p<.05, r=.12$ and the LA sex film $F(1,38)=7.17, p=.01, r=.16$. Although the tolerance of the vaginal pressure increased in the context of sexual arousal, no cross-conditional differences were found in the measured level of intravaginal pressure.

\section{Pleasurable and Painful Pressure}

There was a significant main effect of stimulus type on pleasurable vaginal pressure $\mathrm{F}(2.06,82.44)=30.4, \mathrm{p}<.001, \mathrm{r}=.27$. Contrasts revealed that the ratings of pleasurable vaginal pressure of the HA sexual film $F(1,40)=30.85, p<.001, r=.44$ and the LA sexual film $F(1,40)=15.89, p<.001, r=.28$ were significant higher than the ratings of the neutral film; the ratings of the neutral film $\mathrm{F}(1,40)=9.24, \mathrm{p}<.01, \mathrm{r}=.19$ were significant higher than the ratings of the HA non-sexual film; the ratings of the HA sexual film were significant higher than the ratings of the LA sexual film $F(1,40)=14,27, p \leq .001, r=.26$. There was no significant main effect of condition on painful vaginal pressure. 


\section{Vaginal Pressure and subjective sexual arousal}

Additionally, we explored the influence of vaginal pressure on sexual arousal. A pairedsamples t-test revealed that the HA sexual film with pressure evoked significantly higher levels of subjective sexual arousal $t(1,40)=3.66, p=.001, d=0.57$ than the HA sexual film without pressure.

\section{DISCUSSION}

The current study aimed at testing a new device, the Vaginal Pressure Inducer (VPI), developed to gradually induce increasing vaginal pressure in a standardized and controlled manner in the introitus vaginae. Because this is a first exploratory study, aiming to validate the instrument, we focused specifically on the influence of sexual stimuli on the threshold of unpleasant vaginal pressure and the level of pleasurable and painful vaginal pressure in a sexually functional sample of women. The results confirmed that unpleasantness thresholds as well as ratings of pleasurable vaginal pressure increased in the context of sexual stimuli, when compared to the neutral condition. Comparing HA sexual stimuli to LA sexual stimuli, the results confirmed that HA sexual stimuli are associated with higher ratings of pleasurable vaginal pressure, but HA sexual stimuli were not associated with heightened unpleasantness thresholds of vaginal pressure. Only HA sexual stimuli had a positive effect on the experience of vaginal pressure whereas HA non-sexual stimuli negatively affected ratings of pleasurable vaginal pressure, indicating the specificity of our effects. Furthermore, the results suggested that vaginal pressure in the context of a HA sexual film increased subjective sexual arousal compared to a HA sexual film without pressure. The results showed that gradually increasing vaginal pressure, as applied by the VPI, was well tolerated by the women. The results provide initial support for the VPI as a device to measure the threshold and pleasurable appraisal of vaginal pressure in the context of sexual stimuli. 
The finding that sexual stimuli increased the unpleasantness threshold and pleasurableness of vaginal pressure opposes the pattern of earlier studies showing that sexual arousal increased genital (pain) sensitivity 54,77, while vaginal vibratory sensation 76 and pleasurable ratings remained unchanged 77 . These contradicting findings may partly be attributed to methodological differences across studies. The VPI is the first instrument by which genital pressure can be applied in private by means of a remotely controlled device. Studies thus far required the presence of a research assistant applying the sensory tests. This may have moderated the outcome unintentionally, for example, by inducing heightened self-awareness, distraction from sexual stimuli or negative affect (e.g., shame). Research has shown that female sexual arousal is context-dependent and thus highly influenced by external laboratory cues-166-168. These methodological differences may preclude a direct comparison between the current data of the VPI and extant measurements of genital sensitivity.

Note that the current study is the first study to demonstrate elevated unpleasantness thresholds and ratings of pleasurable vaginal pressure as a function of subjective sexual arousal in a sexually functional sample. This might be a clinically relevant finding, since genuine vaginal pressure apparently is appraised as pain in genital pain patients ${ }^{169}$. It might be interesting to further explore this option by comparing women with and without genital pain in a future study. This may provide targets for intervention and help to develop evidence-based personalized interventions of genital pain. The finding that an increase of subjective sexual arousal is associated with increased ratings of pleasurable vaginal pressure in the context of HA sexual stimuli, compared to LA sexual stimuli, confirms the positive influence of higher levels of subjective sexual arousal on the appraisal of genital pressure sensations. Additionally, indications were found that vaginal pressure may heighten subjective sexual arousal during HA sexual films, which may implicate that vaginal pressure can potentiate sexual arousal in sexually functional women.

The findings illustrate the sensitivity of the VPI to differentiate the level of pleasurableness of vaginal pressure as a function of different arousal levels. This suggests that the VPI seems well suited to assess the influence of several vulnerability factors on the appraisal of vaginal pressure and the relationship with genital pain. The VPI may also be useful for diagnostic and treatment purposes, e.g. learning to associate 
high levels of subjective sexual arousal to gradually increasing levels of vaginal pressure (counterconditioning). The prolonged and gradual increase of vaginal pressure together with the remote control of the VPI, provide a suitable instrument to assess vaginal pressure tolerance in a sexual context.

\section{Limitations}

One limitation of the current study is the experimental design. Considering that sexual arousal in women is highly context dependent, we expected that the experience of vaginal pressure is probably highly related to intrapersonal and relationship factors, which are not included in the current study. To receive more information about the ecological validity of the experienced vaginal pressure, we will ask participant perceptions to which level their experience with the VPI was actually consistent with their experience of vaginal pressure outside the laboratory during intercourse. To increase extern validity of the VPI the influence of partners' presence in the lab will be assessed in future studies.

In order to assess the suitability of the VPI and given the invasive nature of the instrument, we decided to start the experiment with an acclimatization trial followed by a HA sexual film without pressure. Concerning the possibility of a priming effect, the data on the influence of vaginal pressure on (sexual) affect should be interpreted with caution. Future studies should confirm this finding using a fully randomized order of presentation. Additionally, the test -retest reliability of the VPI has to be further investigated.

The current study focused on the affective dimension of pain using a within-subject design by assessing the influence of subjective sexual arousal on the appraisal of vaginal pressure. To prevent eliciting a hypervigilance response towards possible pain sensations in participants, we measured the unpleasantness thresholds and not pain sensitivity by gradually increasing vaginal pressure. To provide more insight in the relationship between the (un)pleasurableness of vaginal pressure and genital pain sensitivity, the VPI could also be used to obtain objective data for pain sensitivity (the 
amount of pumped water, $\mathrm{ml}$; along with the measured intravaginal pressure, $\mathrm{mmhg}$ ) in future studies.

A restriction of the VPI is that the instrument is hard to combine with physical indices of genital arousal. Future research needs to explore other types of physical arousal measures that may be compatible with the VPI, such as oxygenation-temperature method, lubrication, the vaginal pulse amplitude (VPA) to get a more complete picture of the role of sexual arousal in relation to vaginal pressure.

\section{Conclusions}

The current findings indicate the importance of sexual stimuli for the heightening of unpleasantness thresholds as well as the increase of pleasurableness of vaginal pressure. Explicit sexual stimuli fortified the pleasurableness of vaginal pressure paralleled by an increase of subjective sexual arousal. The results provide initial support of the VPI as a device to investigate various determinants of the tolerance and pleasurable appraisal of vaginal pressure in a context of sexual arousal. 


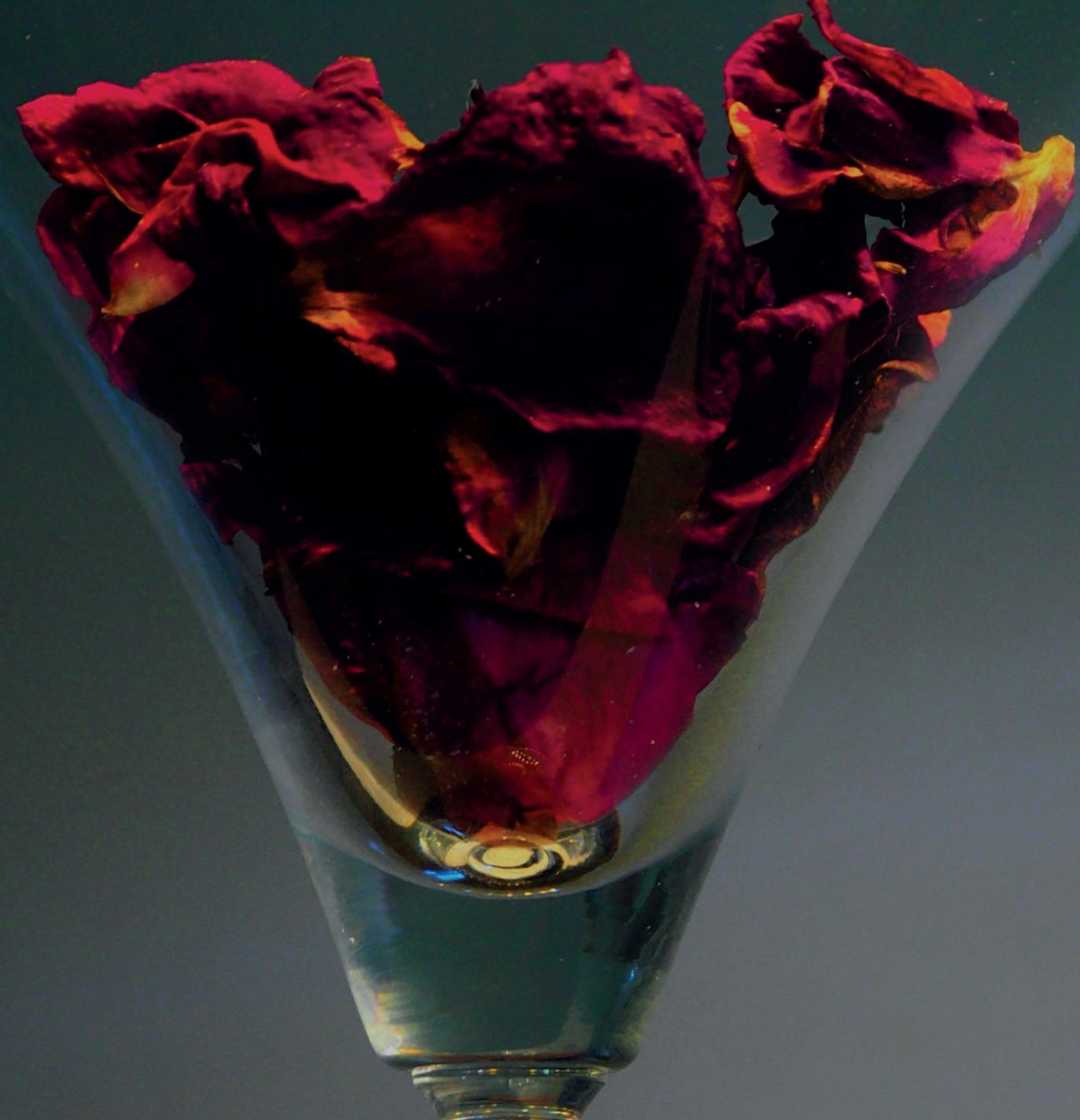

\section{Chapter 6}

Vaginal Pressure: Pain or Pleasure?

The role of individual differences and contextdependent expectations in sexual and nonsexual contexts

R.J. Melles, M.M. Ter Kuile, M.D. Dewitte, M.L. Peters submitted 


\section{ABSTRACT}

Genital pain is associated with increased anxiety, pain vigilance and impaired sexual arousal. The current study aimed to assess the role of these individual differences regarding the appraisal of genital pressure and a possibly mediating role of (pain and positive) expectations in both a sexual and non-sexual context. We induced gradually increasing vaginal pressure in sexually functional women in a neutral acclimatization condition followed by a sexual and a non-sexual condition. In the acclimatization condition anxiety, higher pain vigilance and lower sexual arousability were associated with painful pressure. Pain expectations mediated the relationships of both anxiety and pain vigilance with painful pressure. Associations were found between pain expectations and painful pressure in the non-sexual context and between sexual arousability and pleasurable pressure in the sexual context. The results suggest that higher trait anxiety and pain vigilance and lower sexual arousability increase the vulnerability for pain in first experienced vaginal pressure. Pain expectancies may play a role in persisting and predicting genital pain. Sexual arousability may help to prevent painful and increase pleasurable vaginal pressure. Therefore, to prevent and overcome intercourse pain increasing sexual arousal as well as pain expectancy violation are important targets of both sex education and treatment.

\section{INTRODUCTION}

Intercourse pain is common among women, with prevalence rates around $15 \%{ }^{86}$. Several individual differences are found to be related to intercourse pain. Various studies have shown that, among other things, enhanced distress, anxiety 170-173 pain vigilance $42,71,174$ and impaired sexual arousal during sexual contact may play a role in women with genital pain 31,38,160,175. The impact of these more distal, individual differences in genital pain might be mediated by more context-related, proximal factors like pain expectations. Thus far in the field of genital pain no study has examined the role of pain expectations on painful genital sensations; neither a mediating role of pain 
expectations on genital pain has been assessed. Yet we know from studies in other pain conditions that expectation play an important role in increasing pain intensity ${ }^{176-180}$. In turn, expectations are shaped by individual differences like anxiety and pain vigilance 181-185. Applied to genital pain, we can expect that trait anxiety and pain vigilance increase fearful pain expectations towards intercourse because fear is found to elicit pelvic floor muscle activity 23 and reduce sexual arousal 44,60. The combination of increased pelvic floor muscle activity and reduced sexual arousal results in greater friction between the penis and the vulvar skin, which can result in pain that completes the vicious fear-genital pain circle ${ }^{33}$. There is some evidence supporting the suggested initiating role of anxiety and pain expectations for the genital pain circle. One study found that young women with genital pain reported more anxiety and fear as well as heightened expectations of pain towards their first intercourse. However, because fear and expectations were reported retrospectively, recall bias cannot be excluded ${ }^{174}$. A recent study in women with dyspareunia showed that heightened pain anxiety scores were associated with stronger generalization of pain expectancies towards a "safe" erotic conditioned stimulus ${ }^{36}$. Furthermore, manipulation of pain expectations affected the sexual arousal response showing that heightened pain expectations reduced genital sexual arousal ${ }^{67}$. Taken together, it is plausible that women with higher anxiety- and pain vigilance who experience impaired sexual arousal may be at risk to develop more context- and stimulus-dependent pain expectations, which may increase genital pain.

In addition, for the appraisal of genital sensations, the context, e.g. the presence of sexual stimuli is found to be important for eliciting sexual arousal ${ }^{168}$. Germane to this, higher sexual arousability as well as sexual arousal expectations may prevent genital pain, whereby sexual arousal expectations are likely to mediate the relationship of sexual arousability and genital pain. It is unknown yet whether the association between individual differences and the appraisal of genital sensations depends on the presence of sexual stimuli and whether sexual arousal expectations will be associated with a positive appraisal of genital sensations. The first aim of the current study is to investigate the role of anxiety, pain vigilance and sexual arousability in predicting painful genital sensations, i.e. vaginal pressure and whether the relationship between anxiety, pain vigilance as well as sexual arousability and painful vaginal pressure is mediated by pain 
expectations as well as sexual arousal expectations in both a sexual and non-sexual context.

In a sexual context, vaginal pressure may also induce a pleasurable experience. As sexual stimuli direct our focus of attention and thereby eliciting sexual arousal 1, this pleasurable affective, high attention demanding context may facilitate a positive appraisal of genital sensations 186,187. This fits with the finding of two recent studies showing that women report heightened pleasurableness ratings of vaginal pressure in a sexual context compared to a non-sexual context 188,189 . It is conceivable that also individual differences, especially sexual arousability, may play a role in experiencing pleasurable vaginal pressure. Likewise, positive expectations, i.e. expectations of sexual arousal may mediate this relationship.

To assess the appraisal of genital pressure we used the recently developed Vaginal Pressure Inducer (VPI) ${ }^{188}$. The VPI allows administering a prolonged and dispersed vaginal pressure in the vaginal introitus; exactly there where the penis exerts the largest pressure during penetration and where genital pain is mostly reported. Using the VPI, we tested the role of individual differences as well as proximal context-dependent expectations in the experience of vaginal pressure in terms of pain and pleasurableness, in a sexual and a non-sexual context.

\section{Hypotheses}

We expect that i) the experience of painful vaginal pressure will be positively related to anxiety and pain vigilance and negatively related to sexual arousability in the presence as well as absence of sexual stimulation; ia) pain expectations of the vaginal pressure will mediate these relationships; ib) sexual arousal expectations of vaginal pressure will mediate the relationship between lower levels of sexual arousability and painful vaginal pressure in the presence of sexual stimulation; ii) the experience of pleasurable vaginal pressure will be positively related to sexual arousability; iia) sexual arousal expectations of vaginal pressure will mediate this relationship. 


\section{METHODS}

\section{Participants}

Fifty-five women were interested in participation in the current study. Eleven women were excluded due to: no relationship (8), use of antidepressants (2) and no response (1) (see for exclusion criteria: ${ }^{188}$ ). After inclusion two women dropped out due to illness, leading to a sample of 42 women without sexual problems. One experimental session was terminated prematurely due to problems with the sound when showing the film clips. Consequently, subjective ratings were obtained from 41 women.

The mean age of the 41 women was 24 years (range 17-56, SD 6 years) 51\% had had higher education. Thirty-seven women used hormonal contraceptives. The mean score of the Female Sexual Functioning Index 97,98 was 30.3 (range 19-35; SD=3.4) showing that most women scored within the range of adequate sexual functioning 95 .

\section{Materials}

\section{Stimulus materials}

For the experiment six film excerpts of 5 minutes each were used: one neutral acclimatization film, two explicit sexual films, one erotic sexual film, one emotional nonsexual film and a neutral film. For the aim of the current study, the data of the acclimatization film, the neutral film and one of the two explicit sexual film excerpts were used. For the neutral and acclimatization film, film fragments from a nature documentary of David Attenborough, BBC Life were used. The explicit sexual film excerpts depicted manual and oral sexual and intercourse scenes. The emotional nonsexual film and the low arousal sexual film condition are beyond the scope of the study and are described elsewhere ${ }^{188}$. 


\section{Description of the Vaginal Pressure Inducer (VPI)}

The VPI consists of an inflatable soft rubber balloon placed in a synthetic handle that ends in a flange situated against the vaginal opening, which ensures that the pressure is experienced in the vaginal introitus. The balloon is gradually filled with body temperature water by a pump until a length of 4-6 cm and a maximal circumference of $16 \mathrm{~cm}$ outside the body. When the balloon is filled an outward omnidirectional pressure is given to the surrounding tissues.

To guarantee privacy the VPI is remotely controlled and pieced onto a kind of pants by which it can be administered by the participant herself. The VPI has proven to be valid to examine the appraisal of vaginal pressure as a function of sexual arousal in two recent studies 188,189 .

\section{PROCEDURE}

For a detailed description of the procedure we refer to our prior publication ${ }^{188}$. To acclimatize the participant, the experiment started with the presentation of the neutral film with pressure induction. This was followed by an explicit sex film clip without pressure induction. Next, 4 different film clips with pressure induction were shown in randomized order: an explicit sex film, an erotic film, a neutral film and an emotional non-sexual film. For the purpose of the current study the data of the acclimatization condition as well as the randomized neutral non-sexual film were used for the nonsexual context; the explicit sex film was used for the sexual context. For the entire study two different explicit sex films were used for the condition with and without pressure; which film was paired with pressure was randomized. Consequently for the present study, participants watched one of two different sex films. Data were checked for systematic differences between the two films, but no effect on outcome was present ( $p$ > $.47)$. 
Prior to each condition subjective ratings of expectations were collected. We measured the level of painful, threatening and sexual arousal expectations towards the vaginal pressure. Because threat expectations showed a floor effect, only the painful and sexual arousal expectation scales were used for the present study. Two minutes after the start of the film the vaginal balloon was gradually filled until the vaginal pressure was experienced as unpleasant. Then the participant pressed a button in order to terminate the pressure. Simultaneously the film was terminated followed by subjective evaluations of experienced pain and experienced pleasurableness of the vaginal pressure. To prevent carry-over effects, in between films women completed a letter span working memory task of 5 minutes. The total duration of the experimental session was about $1 \frac{1}{2}$ hour.

\section{Primary Outcome Measures}

Subjective experiences of pleasurable and painful vaginal pressure sensations were measured with the question: "While watching the film to what extent did you experience a pleasurable vaginal pressure on the vagina?" "While watching the film to what extent did you experience a painful pressure on the vagina?" The questions were rated on two seven-point Likert scales ranging from "not at all" to "very strong" (range:1-7). Higher scores indicate higher levels of pleasurable/ painful pressure sensations.

\section{Possible Predictors}

Trait Anxiety was assessed using the well validated trait subscale of the State-Trait Anxiety Inventory (STAI), which consists of 20 items rating anxiety on a four-point Likert scale of 1 (almost never) to 4 (almost always) 190,191. Higher scores indicate greater anxiety, and total scores can range from 20 to 80 . The internal consistency of participants' data in our sample was satisfactory for the TRAIT subscale, Cronbach's $\alpha=$ .81 . 
Pain vigilance for pain sensations was assessed with the Pain Vigilance and Awareness Questionnaire (PVAQ) 192, which contains 16 items rated on a 6-point scale. Higher scores indicate greater pain vigilance, and total scores range between 0 and 90 . The Dutch version of the PVAQ has been reported to be reliable and valid ${ }^{193}$. The internal consistency of participants' data in our sample was satisfactory, Cronbach's $\alpha=.88$.

Sexual arousability was assessed with the four-item sexual arousal subscale of the FSFI rated on a 6-point scale. The internal consistency of participants' data in our sample was satisfactory for the FSFI arousal subscale $(\alpha=.80)$. To correct the negative skewed (1.17) distribution, indicating that the participants had a relatively high score on this subcale, we normalized the distribution using reversed and SQRT transformed scores for the calculations. To increase readability we report higher FSFI arousal scores as higher sexual arousal in the current manuscript.

\section{Possible Mediators}

Subjective expectations of vaginal pressure were measured with the question "What do you expect from the vaginal pressure?" "Not painful/ painful" "What do you expect from the vaginal pressure?" "sexually arousing/ not sexually arousing". Scores were obtained by use of a Visual Analogue Scale (VAS) ranging from 0-100. Higher scores indicate higher pain and higher sexual arousal expectations. To exclude a possible learning effect concerning expectations in all analyses we used the expectation scales of the acclimatization condition s, i.e. when women had not yet experienced the pressure induction.

\section{Statistical analyses}

Descriptive statistics were calculated for all variables. To evaluate whether the contextdependent expectations mediated the relationship of the individual differences with painful pressure, mediation analyses were performed using linear regression analyses. In the first step, painful vaginal pressure was regressed on the putative predictors. 
Separate analyses were carried out with anxiety, pain vigilance and sexual arousability as predictors. In case of a significant relationship between one of these predictors and painful vaginal pressure, the second step added either painful expectation or sexual arousal expectation to the equation. The mediating role of painful expectations was analysed for the acclimatisation condition, the non-sexual as well as the sexual context; the mediating role of sexual arousal expectations was analysed only in the sexual context to fit with our hypotheses. To evaluate whether expectations mediated the relationship between the individual differences and painful vaginal pressure, the standard error of the mediated effect was bootstrapped ${ }^{194}$.

To evaluate whether sexual arousal expectations mediated the relationship of sexual arousal with pleasurable vaginal pressure in the sexual context similar mediator analyses were performed. Pleasurable vaginal pressure was regressed on sexual arousal together with sexual arousal expectations.

\section{RESULTS}

\section{Painful pressure}

Separate regression analyses showed that anxiety $(\beta=0.31, p=.05)$, pain vigilance $(\beta=0.31, p=.05)$ as well as sexual arousability $(\beta=-0.33, p=.03)$ significantly predicted painful pressure in the acclimatization condition. When the mediator pain expectations was included in the regression equation, the relationship between anxiety and painful pressure $(\beta=0.22, p=.15)$ and between pain vigilance and painful pressure $(\beta=0.19$, $p=.22)$ as well as the relationship between sexual arousability and painful pressure $(\beta=-$ $0.28, p=.06$ ) were no longer significant. When the standard error of the mediated effect was bootstrapped, pain expectations proved to be a significant mediator of the relationship between anxiety and painful pressure $(\beta=0.35, p=.02$ (95\% [BCI.0008$.0418])$ and of the relationship between pain vigilance and painful pressure $(\beta=0.34$, 
p=.03 [BCI.0002-.0407]), but pain expectations was not a significant mediator of the relationship between arousability and painful pressure (95\% BCI -1.4384-.3539=ns).

In the randomized non-sexual context, separate regression analyses indicated that anxiety, pain vigilance and sexual arousability did not significantly predict painful pressure $(\beta<0.24 ; p>$.14). Because the individual difference variables were not associated with painful pressure in this condition, the mediation analyses were omitted. However, we did regress painful pressure on pain expectations per se and found a significant association between pain expectations of vaginal pressure with painful pressure $(\beta=0.33 ; p=.04)$.

In the sexual context, separate regression analyses indicated that anxiety, pain vigilance and sexual arousability did not significantly predict painful pressure $(\beta \leq 0.25 ; p \geq .12)$. However, we did regress painful pressure in the sexual context on pain expectations per se and found a marginally significant association between pain expectations of vaginal pressure with painful pressure $(\beta=0.3 ; p=.056)$.

\section{Pleasurable pressure}

Separate regression analyses indicated that sexual arousability significantly predicted pleasurable vaginal pressure $(\beta=0.31, p=.049)$ in a sexual context. This association did not appear to be mediated by expectations. When pleasurable vaginal pressure was regressed on sexual arousal together with pain or sexual arousal expectations, neither pain expectations $(\beta=0.13 ; p=.4)$ nor sexual arousal expectations $(\beta=0.10 ; p=.56)$ was significantly associated with pleasurable vaginal pressure. 


\section{DISCUSSION}

The current study aimed to assess the role of trait anxiety, pain vigilance, sexual arousal and expectations in the experience of vaginal pressure and to explore whether these associations are mediated by (both pain and sexual arousal) expectations. We tested the association between these predictors and the appraisal of vaginal pressure in the acclimatisation non-sexual condition, as well as in two randomized conditions presenting sexual and non-sexual stimuli. In line with our expectations, the results confirmed the predictive value of trait anxiety, pain vigilance and lower sexual arousability for genital pain sensations, yet only in the acclimatisation condition where no sexual stimulation is presented. Pain expectations were found to mediate the relationships between anxiety as well as pain vigilance and painful vaginal pressure. In the subsequent non-sexual and sexual conditions individual differences were not associated with painful pressure. However, pain expectations were significantly and positively associated with painful pressure in the non-sexual condition, and marginally significantly and positively associated with painful pressure in the sexual condition. Sexual arousal expectancies were not associated with painful pressure. Sexual arousability was significantly and positively associated with pleasurable vaginal pressure.

This is the first study to examine the role of trait anxiety, pain vigilance and sexual arousability regarding the appraisal of induced genital sensations in both a sexual and non-sexual context. Associations between trait anxiety, pain vigilance and lower sexual arousability and painful genital sensations were observed in the acclimatization nonsexual condition only. This was expected as higher trait anxiety and pain vigilance may provoke pelvic floor muscle tension as well as impaired sexual arousal leading to genital pain 2. The current finding corroborates other findings showing an interrelationship between these variables and genital pain 38,71,170,171,174. However, in the subsequent nonsexual and sexual condition no relationships were observed between individual differences and painful appraisals. This might suggest that individual differences play a more prominent role with novel experiences where people do not know what to expect. Under these circumstances, habitual tendencies may more strongly shape expectancies and subsequently also the actual experiences of people. Apparently, the repeated 
exposure to the vaginal pressure over the course of the experiment may have comforted the participants and overruled their pre-existing apprehension.

In contrast to the absence of an effect of more stable individual difference variables, pain expectations were associated with painful pressure in the non-sexual conditions. In the acclimatization trial, where participants were exposed to the vaginal pressure for the first time, pain expectations mediated the relationships between anxiety/ pain vigilance and painful pressure. In the subsequent non-sexual condition pain expectations seemed to persist and predict painfull vaginal pressure, as pain expectations were significantly and positively related to pain ratings in the non-sexual context. This underscores the important role of pain expectations as previous studies emphasize an important role of pain expectations to predict pain.

In the sexual condition pain expectations were marginally related to pain ratings. It is conceivable that the presence of sexual stimuli may have directed the focus of attention, thereby distracting the attention away from possibly pain-related expectations. Together with the elicited sexual arousal, commonly experienced as a highly pleasurable affect, the sexual context may facilitate a positive appraisal of genital sensations. In other words: the high attention demanding sexual context may overrule a possible preexisting apprehension, especially because the participants had no genital pain disorder as the present study is conducted in a sexually functional population.

Together these findings suggest that tackling pain expectations may be an important target of treatment. Several process-related studies have indeed established that targeting expectations is feasible and can improve clinical outcomes. In the field of nonsexual pain, a meta-analysis showed that patients' pain can be relieved using interventions that tap into pain expectation 176. In studies on anxiety, increasing evidence shows that 'belief disconfirmation' e.g. expectancy violation is important to overcome anxiety (see for an overview of studies: 195. This fits with the clinical experience applying therapist-aided exposure treatment in women with vaginismus 196. Women with vaginismus report high levels of genital pain and pain/ penetration anxiety 25 resulting in an involuntary spam of the pelvic muscles which hinders vaginal penetration. During therapist-aided exposure therapy expectancy violation towards vaginal penetration is one of the most important targets of treatment. Therapist-aided 
exposure has proved to be highly effective with success ratings of $89 \%{ }^{58,59}$ and the therapeutic outcome was mainly mediated by changes in catastrophic and pain related penetration beliefs ${ }^{196}$. Together these findings suggest that (modifying) expectations of genital pain sensations may predict (release of) genital pain. Moreover, the observed predictive role of the more proximal pain expectations for genital pain may question the influence of the commonly given instruction to report the level of experienced pain to participants prior to sensory testing in experimental studies. This may introduce an unintentional effect of expectation of pain which may increase pain sensitivity. Measuring both painful and positive appraisals in sexual and non-sexual contexts in experimental studies may prevent this effect.

The finding that sexual arousability predicted lower rates of painful vaginal pressure in a non-sexual context together with higher rates of pleasurable vaginal pressure in a sexual context can be explained by a positive associative learning process. Research has shown that enhancing pleasurable stimulation and sexual arousal may decrease pain by mitigating pain sensitivity during sexual activity 77,197,198. These findings are consistent with recent studies showing increased (state) sexual arousal to be associated with decreased painful - and increased pleasurable vaginal pressure ${ }^{188,189}$. The absence of an association of sexual arousal expectations and painful as well as pleasurable vaginal pressure may be attributed to our decision to use the first expectation ratings only, in order to avoid that expectations would reflect the actual experience of pain and sexual arousal in previous trials. It is likely that vaginal pressure may elicit sexual arousal expectations only when sexual stimuli are expected. Our results suggest that positive experiences with sexual arousal may prevent painful and increase pleasurable vaginal pressure.

\section{Limitations}

The present study has several limitations. First, to prevent negative conditioning participants were instructed to stop the vaginal pressure as soon as it felt unpleasant. This may have reduced pain expectations as well as pain ratings. In addition, the study is conducted in a sexually functional population, which impedes any firm conclusion to be 
drawn on genital pain. Women with genital pain may have learned to associate genital pressure with pain and they usually experience the genital pain in a sexual context. Future research should include women with and without genital pain to explore the influence of these different learning processes on the appraisal of genital pressure in both a sexual and non-sexual context.

Another limitation of the present study is that a learning effect may have influenced the outcome as the acclimatization trial was prior to the randomized conditions. This may have prevented finding a significant association between individual differences and painful appraisals in the randomized sexual and non-sexual conditions.

\section{Conclusions}

The current study suggests that to prevent and overcome intercourse pain interventions should focus more on increasing sexual arousal and modifying pain expectations towards (first) intercourse. Both can be effectuated by sex education as well as cognitive behavioural treatment. 


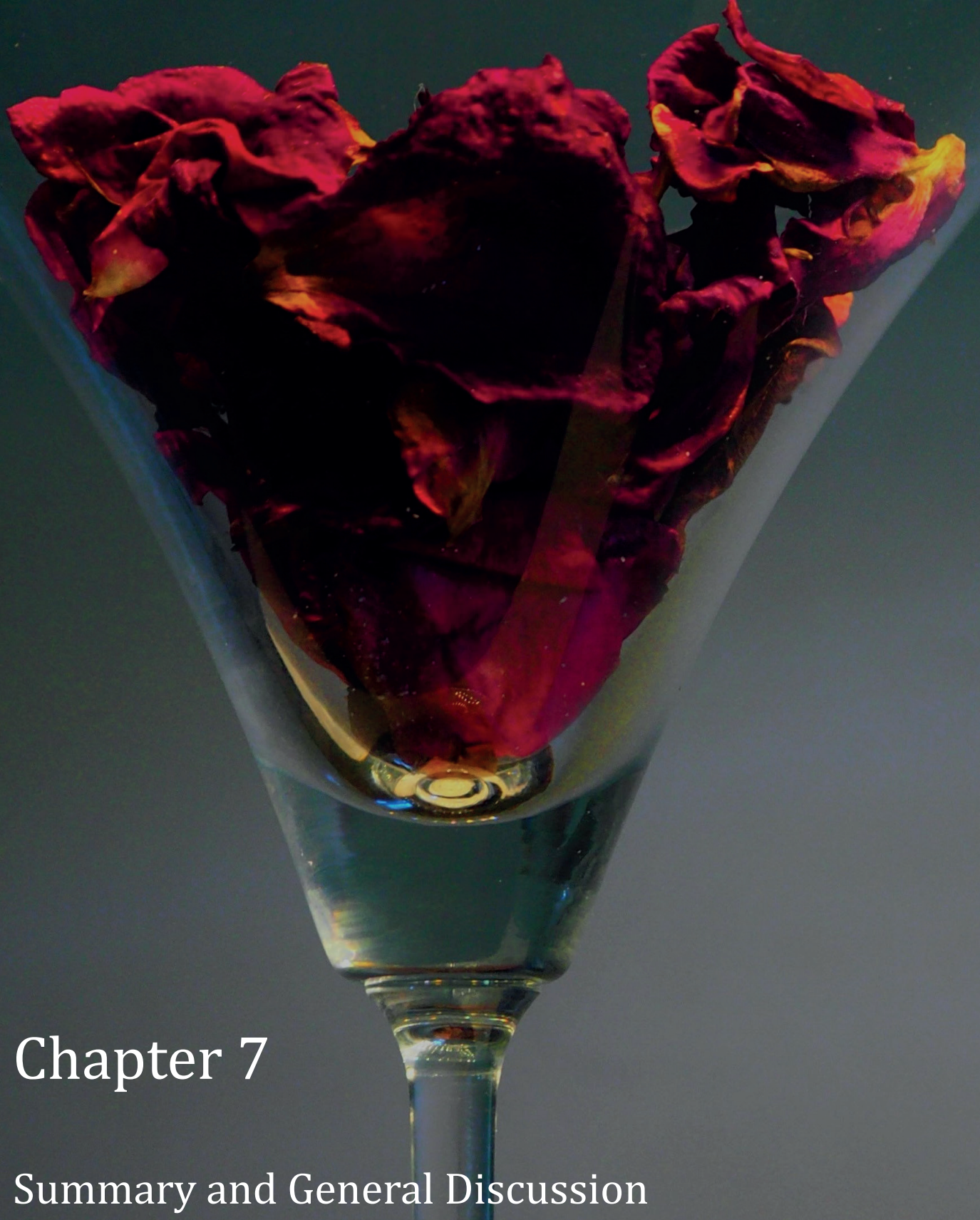




\section{Introduction}

The aim of the research reported on in the current thesis was to explore both psychological risks and resilience factors related to painful or pleasurable vaginal penetration.

Based the information processing model of sexual arousal and the cognitive behavioural model of genital pain, we investigated the potential role of attentional bias and implicit and explicit negative associations with erotic stimuli. Furthermore, we explored the value of sexual arousal for a positive appraisal of vaginal pressure. We conducted five experimental studies. In this concluding chapter we first summarize the findings of the studies, and then discuss them in relation to the Cognitive Behavioural (CB) model of genital pain and the cognitive processing model of sexual arousal, both of which have been presented in chapter 1 . We will address the limitations of the studies and discuss suggestions for future research. The chapter ends with a discussion of implications for the treatment of female genital pain.

\section{Summary of the studies}

The first study (Chapter 2) investigated whether increased attentional bias for sexrelated threats (e.g, pain) and less implicit incentive valence ("wanting") may play a role in the impairment of sexual arousal and the experience of genital pain. We also assessed if differences in threat and incentive processing may help to explain the greater persistence of coital avoidance in women with vaginismus compared to those with dyspareunia. For this purpose, three groups of women (vaginismus, dyspareunia and controls) completed a Visual Search Task (VST) to assess attentional bias, and a singletarget Implicit Association Test (st-IAT) to measure implicit "threat and wanting" associations regarding vaginal penetration stimuli. No group differences were found in attentional bias or implicit associations. Furthermore, correlational analyses showed that delayed detection of sex stimuli and stronger implicit threat associations were related to lower sexual arousal in daily life in all women. These findings do not corroborate the view that attentional bias for pain or sex contributes to genital pain, or 
that differences in coital avoidance can be explained by differences in attentional bias or implicit threat/incentive associations. However, the correlational findings are consistent with the view that implicit threat associations and impaired attention for sex stimuli may interfere with the generation and development of sexual arousal.

In the next study (Chapter 3) we explored the role of negative general affective valences of erotic stimuli in women with vaginismus. To this aim, global affective associations with erotic (non-penetration) stimuli as well as vaginal penetration stimuli were assessed in women with vaginismus and control women. We additionally explored group differences regarding implicit and explicit threat associations with sexual penetration stimuli. Implicit threat associations were assessed using a version of the stIAT comparable to that used in Chapter 2. To test global implicit affective associations we used the Affective Simon Task (AST). We found that, compared to controls, women with vaginismus showed relatively stronger threat associations and less positive global affective associations with sexual stimuli at the explicit level but not at the more implicit level. These findings suggest that explicit threat and negative global affective appraisals are both involved in vaginismus. Hence, the studies presented in Chapters 2 and 3 found no evidence for the involvement of implicit threat, incentive or negative global affective associations with sexual stimuli in women with genital pain.

We also examined the effect of therapist-aided exposure treatment on the implicit and explicit threat and global affective associations with erotic stimuli in women with lifelong vaginismus (Chapter 3). Results showed that therapist-aided exposure treatment was effective in reducing the explicit threat associations and in amplifying the explicit positive global affective associations with sexual stimuli, whereas the implicit associations remained unaffected.

Chapter 4 reports on a study to assess the mechanisms of change in therapist-aided exposure treatment in vaginismus. We found that treatment outcome (coital frequency, symptoms of vaginismus and coital pain) at 12 weeks was mediated by changes in negative and positive penetration cognitions at 6 weeks, which was mainly driven by the increased reduction of catastrophic pain penetration cognitions.

The last two studies were designed to test the assumed role of sexual arousal in preventing and overcoming genital pain. For this purpose, a new instrument, the Vaginal 
Pressure Inducer (VPI) was developed to simulate the vaginal sensations during penetration and to match the experimental setting as closely as possible to the context in which these sensations naturally occur. The study presented in Chapter 5 aimed to achieve a better understanding of the influence of sexual arousal on the appraisal of vaginal pressure sensations in sexually functional women. Using the VPI, we administered gradually increasing vaginal pressure in neutral, erotic and explicit sex conditions. Results showed that women had higher unpleasantness thresholds in a sexual context compared to a non-sexual context. Moreover, ratings of pleasurableness were higher in the sexual compared to the neutral context, and while watching an explicitly sexual film. Indications were found that vaginal pressure can potentiate sexual arousal in sexually functional women, as vaginal pressure seemed to increase sexual arousal while watching explicitly sexual films. These results confirm the importance of explicit sexual stimuli for the tolerance and pleasurableness of vaginal pressure. Furthermore, the findings provide some initial support for the suitability of the VPI to study determinants of pleasant and unpleasant appraisal of vaginal pressure.

In our final study (Chapter 6) we explored predictors of the appraisal of vaginal pressure as induced by the VPI in the same sample women who took part in the previous study. We investigated the role played by individual differences (e.g., increased trait anxiety, pain vigilance and impaired sexual arousal in daily life) in the appraisal of vaginal pressure, in both a sexual and non-sexual context. We also explored a possible mediating role of pain and pleasure expectations. In line with our expectations, the results confirmed the predictive value of trait anxiety, pain vigilance and reduced sexual arousability for genital pain sensations, yet this was only true in the acclimatization condition (i.e., the first experience of vaginal pressure) when no sexual stimulation was presented. Pain expectations were found to mediate the relationship between anxiety and painful vaginal pressure, as well as that between pain vigilance and painful vaginal pressure. In the remaining conditions, significant and positive associations were found between pain expectations and painful vaginal pressure in a non-sexual context, and between sexual arousability and pleasurable vaginal pressure in a sexual context. We concluded that reducing pain expectancies and increasing sexual arousal are important targets of treatment to prevent and overcome intercourse pain. 


\section{Conclusions}

When evaluating the results presented in the current thesis in terms of the main research question formulated in Chapter 1, i.e. Which implicit and explicit cognitive processes underlie painful or pleasurable vaginal penetration in women?, the following answers can be generated:

The findings of our studies suggest that the involvement of negative appraisals in female genital pain is restricted to the explicit level (Chapters 2 and 3). The increased explicit threat and negative global affective associations with sexual stimuli were successfully altered by therapist-aided exposure treatment of women with vaginismus (Chapter 3). Most couples (90\%) were able to have intercourse after this treatment, and the outcome (in terms of coital frequency, symptoms of vaginismus and coital pain) was mediated by a pronounced reduction of catastrophic pain penetration cognitions (Chapter 4).

Evidence was obtained for the importance of high levels of sexual arousal for a positive appraisal of vaginal pressure. Painful vaginal pressure was influenced by individual differences, in particular by pain expectancies, but only in the absence of sexual stimuli. Pleasurable vaginal pressure was influenced by increased sexual arousability.

\section{Theoretical implications and general reflections}

The studies presented in the current thesis contribute to a better understanding of negative cognitive processes in genital pain and their modifiability, and provide preliminary evidence for the value of sexual arousal in obtaining a positive appraisal of vaginal pressure, which may be important to prevent genital pain. In this section, we discuss the findings in relation to three themes. The first concerns the lack of attentional bias and/or negative implicit associations with sexual stimuli in women with genital pain. Secondly, we argue that, in addition to reducing negative explicit appraisals, bolstering positive explicit appraisals may also be important to overcome female genital pain. The third theme concerns the importance of sexual arousal for a pleasurable appraisal of vaginal pressure sensations. 


\section{Role of attention and implicit appraisals}

The studies presented in Chapters 2 and 3 provided no evidence for a crucial role of attentional bias and implicit threat or negative global affective associations with sexual stimuli in female genital pain. Our findings are in agreement with the results of another study that found no implicit sex-threat associations in women with genital pain 39 . Drawing on the dual process model, which differentiates between implicit and explicit processes, it has been assumed that implicit appraisal is linked to genital arousal, while explicit appraisal is linked to subjective arousal. Previous lab research has shown that genital arousal is not impaired in women with genital pain 46,54,60,68. This is in line with our results, which showed no difference in implicit appraisal between women with and without genital pain. One explanation for these findings would be that, in the absence of fear, the implicit responses to pain-related stimuli may have retained a predominantly sexual meaning, possibly protected by the reproductive value of sex and a positive reinforcement of sexual responses.

In both women with and without genital pain, reduced subjective sexual arousal was related to both delayed detection of sex-relevant target words and stronger sex-threat associations (Chapter 2), which confirms the content validity of the tasks we used. Combining this with the finding that the genital pain group reported lower levels of sexual arousal than the control group (Chapter 2), it is possible that the visual stimuli we used in our research did not sufficiently capture the relevant aspects that women with genital pain actually experience or fear during sex. For example, the expectation of genital pressure could more adequately trigger implicit threat appraisals. The absence of implicit appraisals may mean that the lab context is not salient enough for the women with genital pain to attribute a threatening meaning to the stimuli. In the field of social psychology, implicit measures have shown a high level of context sensitivity (e.g., mood states, see for an overview ${ }^{199}$ ). Other stimuli (e.g., learned context-related pain cues like engaging in coitus with the partner) that are present at home and not in the laboratory may tap into the specific fear that symptomatic women may experience, which, as such, would lead to attentional bias, implicit threat appraisals as well as impaired genital arousal in private encounters. Further support for this resides in the finding that, in both women with and without genital pain, relatively strong implicit "sex-threat" associations were related to relatively weak implicit "sex-wanting" associations (Chapter 2). This 
confirms the view that a high threat value of sex may interfere with an individual's readiness to have sex, and hamper the generation of sexual arousal. Since cognitive and motivational appraisals may be regarded as proximal determinants of sexual behaviour, this may explain why women with genital pain show high levels of avoidance of intimacy and of activities that elicit sexual arousal 5652 , and that they report reduced sexual desire 37,173,200 38. More specifically, since intimacy or sex-related activities will trigger threatrelated associations, this may lead to avoidance of potential sex cues, which in turn further impairs sexual desire.

Not only the stimuli, but also the attributes we used may have insufficiently captured the concerns of women with genital pain. The terms we used, i.e., "threat/safe, positive/negative and wanting/not wanting", may not have sufficiently represented the affective and motivational valences of sex/vaginal penetration for women with genital pain. Implicit associations are assumed to reflect general cultural stereotypes that are conveyed through socialization 201. Women are bombarded with images of women's sexual submission and subservience to male partners ${ }^{202}$, which reflects social gender norms towards sex and sexual behaviour 202,203. Accordingly, research has shown that women automatically associate sex with submission 202. These implicit associations were found to be related to greater personal adoption of a submissive sexual role and, consequently, lower levels of sexual arousal 202. Another study found that women with genital pain automatically perceived themselves as sexually less competent. When this negative actual self-concept did not match their implicit ideal sexual self (i.e., being sexually competent from the perspective of the partner), this was found to lead to more genital pain, fear of pain, and a stronger tendency to continue with sex despite the pain 204. Women's implicit appraisal of painful vaginal penetration (or the absence of penetration), in itself has not yet been explored. A few recent studies have explored explicit valences of vaginal penetration in young women with genital pain. Vaginal penetration was evaluated as "of great importance to the romance in a relationship" and "an affirmation of being an ideal woman", but was also associated with "duty/pressure, resignation, sacrifice, and feeling guilt". Refusing vaginal penetration was associated with "being foolish, inadequate, or being offended" 52,205. Further research is needed to explore if implicit sex associations reflect the influence of social gender norms in female genital pain. 


\section{Explicit appraisals}

Chapter 3 reported stronger explicit associations between sex and fear in women with vaginismus, and how the strength of these "sexา-fear" associations was reduced following therapist-aided exposure treatment. These findings confirm the (maintaining) role of fear avoidance in genital pain suggested by the CB model ${ }^{2,33}$. Chapter 3 also further substantiated the effectiveness of therapist-aided exposure treatment, as demonstrated by the reduction of fear and avoidance. Following this treatment, 89\% of the couples reported coitus during the first 2 weeks (Chapter $4{ }^{59}$ ).

Chapter 4 described mechanisms of change regarding treatment outcome (in terms of coital frequency, symptoms of vaginismus and coital pain) in vaginismus. Indications were found that the treatment outcome was mediated by changes in both catastrophic pain/penetration cognitions and perceived control cognitions. Additional support for the $\mathrm{CB}$ model stems from the finding that it was particularly the reduction of catastrophic pain/penetration cognitions which was positively related to the improvement of all of three outcome measures. However, we cannot draw conclusions on the temporal order of changes. Our findings may also suggest that cognitions and/or "sex-fear" associations were mediated by changes in vaginal penetration (or the avoidance thereof), and a reduction of pain (or the fear of it). This fits in with the findings of a recent study of women with dyspareunia, which showed that avoidance behaviour mediated the relation between pain catastrophizing and genital pain 51 .

Although therapist-aided exposure is effective in reducing fear, no treatment effects were found regarding sexual pleasure ${ }^{58,59}$. Moreover, about half of the participants still reported some pain or discomfort during intercourse at three months' follow-up ${ }^{59}$. The absence of an improvement of sexual pleasure (like desire, sexual arousal and orgasm) fits in with previous findings that therapist-aided exposure treatment did not improve sexual satisfaction 58. On the one hand, these findings can be explained by the treatment's focus on the emotional and behavioural changes needed to enable vaginal penetration. On the other hand, the therapists did pay explicit attention to improving the sexual relationship by means of a follow-up trajectory of two sessions that encouraged couples to maintain vaginal penetration (to further reduce fear), but also to increase sexual pleasure and to link vaginal penetration to sexual arousal. Subsequently, three 
additional sessions of sex therapy to improve sexual pleasure were offered to all couples who were able to have intercourse (89\%). More than $40 \%$ of them chose to participate in these additional sex therapy sessions. Even this group, however, did not show increased sexual pleasure. We may conclude that our exposure treatment contributes to reducing negative appraisals, but, unfortunately, is yet unable to boost sexual pleasure.

Our results corroborate those of other studies. Effect studies of cognitive behaviour therapy (CBT) in dyspareunia have shown that, after treatment, a significant group of women still report intercourse pain (31\% -67\%) and impaired sexual pleasure (29\%37\%) 56,206-209. The literature on chronic pain has also reported the limits of altering a negative affective valence of stimuli into a positive one ${ }^{210}$. This can be explained by referential (i.e., evaluative) conditioning, which seems relatively resistant to extinction 79. In referential conditioning, an affective evaluative reaction evoked by a unconditioned stimulus (US) is transferred into a conditioned stimulus (CS), as a result of their contingent presentation ${ }^{79}$. Despite the suggested rigidity of evaluative learning, studies of disgust and chronic pain have revealed that counterconditioning may affect evaluation-related valences of stimuli 211-217. Counterconditioning has not yet been investigated with regard to genital pain.

Several more recent approaches have incorporated emotion regulation into CBT. One feasibility study integrated emotion regulation into the standard exposure treatment, by focusing on identifying personal values and important personal goals, emotion experiencing, flexible problem solving and coping skills to achieve important personal goals 218 . Preliminary findings suggest that this approach can reduce fear-related and emotionally distressing chronic pain.

In the same vein, positive psychology focuses on resilience by, among other things, strengthening engagement in meaningful activities and positive expectations. Numerous studies in positive psychology have found favourable effects on outcomes like mood, anxiety, well-being, health, physical activity and, to a lesser extent, pain 219-225.

Both mindfulness and acceptance and commitment therapy (ACT) focus on the acceptance of distressing emotions and on important personal values and goals. In female genital pain, mindfulness-based CBT appears promising to target both pain and sexual suffering from genital pain $200,226,227$. In the case of genital pain, the use of 
mindfulness during dilator insertion has been suggested, combining the act of using vaginal inserts with an enhanced emotional awareness. This approach aims to promote a positive and autonomous experience of vaginal penetration, which could lessen feelings of obligation, disassociation and resentment 228 .

\section{A social/dyadic perspective on female genital pain/pleasure}

From a social/relational perspective on coping with genital pain, personal goals and motives of sexual behaviour seem important to regulate emotions in women with genital pain. Firstly, sexual goals seem to be influenced by social gender norms, which suggest an ideal image of a sexual woman, who is willing and able to have intercourse (even despite pain) 52,56,202,203,205,229. Women with dyspareunia reported feeling pressure to meet these internalized social gender norms 84,204 . It may be difficult for women to meet such norms, as intercourse is not the most sexually stimulating activity for women 230,231. The (social and medical) validation of the reproductive value of female genitals (vagina) corroborates the predominant male model of sexual responding that is directed towards intercourse (often in quantitative rather than qualitative terms), thereby neglecting the value of the clitoris (and the inner parts of it!) for sexual pleasure 232,233. In light of this social context, it is not surprising that women who are not able to have (pain-free) intercourse show a more negative sexual self-image and less sexual autonomy than sexually functional women $35,52,175,234$.

Secondly, sexual goals are related to the relational context (i.e., dyadic motives), partner responses and vaginal penetration behaviour in female genital pain. Following theories of social motivation 235 , Rosen and colleagues conceptualized approach goals as reasons for pursuing desirable relationship outcomes (like intimacy). Approach goals have been linked to reduced pain and increased relationship satisfaction, sexual satisfaction and sexual desire 236-239. Persistence behaviour towards painful coitus has been linked to avoidance goals, aiming to prevent negative relationship outcomes such as partners' disappointment $52,74,84,205,240$. These avoidance goals are associated with greater pain (or the fear of it) 239,241 and lower reported satisfaction and desire 236,238,242,243 . 
Note that these studies on the impact of social norms and the influence of dyadic processes on genital pain are mainly based on women with dyspareunia. However, both women with vaginismus and women with dyspareunia reported less pleasure and physical desirability motives to engage in vaginal penetration ${ }^{52}$. We do not know whether this might be a consequence of genital pain, and/or whether this may relate to internalized social norms.

In clinical practice, many women with vaginismus report being motivated to start therapist-aided exposure treatment, despite feelings of embarrassment, by their wish to be a "normal" woman (i.e., being able to have intercourse). Further research is needed to explore if (and if so, which) social norms, dyadic processes and sexual goals are related to fear of coitus (or its persistence) and impaired levels of sexual pleasure in women with vaginismus, and how this could be affected by treatment.

\section{A psychosomatic perspective on the importance of sexual}

\section{arousal}

Given that the experience of sexual arousal is assumed to prevent and overcome genital pain as well as promote pleasurable intercourse, it is surprising that thus far, few attempts have been made to design a (positive) sexual context to assess genital sensations (including pressure). To test the assumed role of sexual arousal in preventing and overcoming genital pain, it is essential to simulate the vaginal sensations during penetration and to match the experimental setting as closely as possible to the context in which these sensations naturally occur. For this purpose, we have developed a new instrument, the vaginal pressure inducer (VPI). The VPI enables a prolonged and dispersed vaginal pressure to be administered in the introitus vaginae. It is precisely these pressure sensations which, in the case of genital pain, are likely to be appraised as potentially painful. With the VPI, vaginal pressure sensations can be applied in a controlled and standardized way, increasing the internal and external validity of laboratory research on genital pain. The VPI enabled us for the first time to demonstrate the importance of explicit sexual arousal for positive appraisal and increased tolerance of vaginal pressure (Chapter 5). 
Moreover, indications were found that individual differences, i.e., higher anxiety and pain vigilance as well as lower sexual arousability, may increase the risk of developing more stimulus-dependent pain expectations, which in turn mediate the relationship with painful vaginal pressure (Chapter 6). The presence of sexual stimulation seemed to overrule a possible preexisting apprehension (i.e., increased trait anxiety and hypervigilance) in a sexually functional population. Sexual arousal may facilitate that the genital pressure sensations become part of the sexually stimulating event, allowing a conscious positive appraisal (Chapter $5{ }^{189}$ ). Several findings presented in this thesis may indicate the role of sexual arousal in facilitating positive cognitive processes. Firstly, higher levels of sexual arousability were related to both a more rapid detection of sexual stimuli and lower sex-threat associations in both women with and without genital pain (Chapter 2). Secondly, the automatic appraisals of sexual stimuli may have retained a predominantly sexual meaning in women with genital pain, as no evidence of more negative implicit appraisal was found (Chapters 2 and 3). Thirdly, the presence of sexual stimuli (state sexual arousal), as well as higher sexual arousability, were found to facilitate the pleasurableness of vaginal pressure in sexually functional women (Chapters 5 and 6). Taken together, these findings can be explained by a positive associative learning process. In accordance with the information processing model, the genital sensations are matched with memories in the mind. Highly arousing sexual stimuli (or memories of such stimuli) then facilitate a positive appraisal of genital sensations. These findings may indicate that, even in women with continuous genital pain symptoms, experiencing sexual arousal stimuli may have the potential to facilitate positive cognitive processes, which in turn facilitate the pleasurableness of vaginal penetration. Taken together, these findings suggest that higher levels of sexual arousal seem relevant to prevent genital pain.

\section{Limitations and strengths}

\section{Limitations of the studies}

The studies presented in the current thesis have a number of limitations. Several limitations have already been discussed in the discussion sections of the respective 
chapters. Limitations related to the appraisal studies have been described in the general reflections presented earlier in this chapter. Briefly, the lab situation may not have been salient enough and the stimuli may have been too global, thereby affecting implicit appraisals, which are known to be context-dependent. Some additional limitations, mainly related to the VPI studies, are considered below.

One limitation of the VPI studies is that we have so far not succeeded in combining the VPI with physical indices of genital arousal. Our efforts to use the labia thermistor clip in combination with the VPI failed because the temperature of the water that is used to inflate the vaginal balloon confounded the labial temperature measurements. Future research is needed to explore other measures of physical arousal that may be compatible with the VPI, such as the oxygenation-temperature method or the vaginal pulse amplitude (VPA), in order to get a more complete picture of the role of sexual arousal (i.e., both subjective and genital arousal) in relation to vaginal pressure.

A second limitation is that even though the VPI is suitable for objective measurement of the activity of the pelvic floor muscles by means of the pressure gauge, it is not able to differentiate between the activities of the superficial and deeper layers of pelvic floor muscles. Very little is known about the activity of the pelvic floor muscles during sexual arousal 64,65,244. Preliminary findings suggest that it is specifically the superficial pelvic floor muscles which seem to be activated by threatening (pain) stimuli, and may relax during initial states of sexual arousal; contractions of the deeper layers of pelvic floor muscles have been measured during higher states of sexual arousal 61. More knowledge about the specific activity of the pelvic floor muscles, the interrelationships with emotional stimuli and the effects on the pleasantness or unpleasantness of vaginal pressure may help to understand sexual pain.

A third limitation of the vaginal pressure studies presented in the current thesis is that they do not allow any firm conclusion on genital (i.e., intercourse) pain. Firstly, these studies did not include a clinical sample of women reporting genital pain. Although we found several factors associated with unpleasant vaginal pressure, these findings were based on sexually functional women. Secondly, we did not measure the vaginal pain thresholds, but only the threshold of unpleasantness. Participants were instructed to stop the vaginal pressure as soon as it felt unpleasant. 
In addition, the VPI does not allow vaginal sensations to be measured during thrusting, which is a characteristic of vaginal penetration. Although the VPI aims to accurately reflect the more pronounced and prolonged pressure during masturbation and partner sex, it lacks the rub effect of genital stimulation, and we did not ask the participants about the extent to which the sensations corresponded with coitus. Future studies need to specify which genital sensations are elicited by the VPI and how and to what extent they match those during vaginal penetration.

A general limitation of all presented studies is that the generalizability of the results may be affected by volunteer bias. The control samples consisted of volunteers who selfselected to participate on the basis of advertisements at the university and in the local media. Although all control groups of the studies presented in the current thesis scored within the normal range with respect to sexual functioning (as measured by the Female Sexual Function Index, FSFI), participants in sex research have been found to report a more positive attitude towards sexuality, less sexual guilt and sexual fear, and more sexual experience than those not taking part ${ }^{245-247}$. Note that this limitation does not affect the clinical studies (Chapters 2, 3 and 4), as the genital pain patients we recruited were mainly regular patients of the outpatient clinic of sexology referred by their gynaecologist or general practitioner.

A final reflection is that the studies and clinical interventions presented in the current thesis focused mainly on measuring functional behaviours, like the possibility of vaginal penetration, reduction of genital pain, fear and catastrophizing cognitions and the improvement of sexual functioning. This might give the impression that a functional genital response is the main goal of research and clinical treatment. However, the overall goal of sexual interactions is increased satisfaction and sexual pleasure, rather than a functional genital response or a "normal" sexual performance.

\section{Strengths of the studies}

The current thesis presented a series of systematic studies of the determinants and underlying mechanisms of sexual arousal in the context of genital pain. Implicit tasks as 
well as explicit reports were used to assess psychological processes like attention and appraisal.

It is important to note that the paradigms for measuring attention and implicit appraisals (e.g., the AST and the Visual Search Task) were promising in the days when we carried out our studies, but have been criticized in more recent studies 248,249. Nowadays, alternative paradigms have been suggested (e.g, eye-tracking) ${ }^{250}$. We do want to emphasize, however, that when we developed these studies, the assessment of attention and implicit cognitive processes in sexual dysfunctions was unexplored territory. Hence, this series of studies reflects pioneering work.

An important strength is that we used systematic comparisons between well-matched clinical groups and control groups. The differentiation between primary vaginismus and dyspareunia was based on clear criteria, which helped us study their unique features (e.g., the exclusive role of targeting avoidance behaviour towards intercourse). The clinical samples were also substantial, and recruiting them took a lot of effort, in view of the strong avoidance tendencies in women with vaginismus. The fact that we included women with vaginismus (which is a clearly understudied population when compared with research on dyspareunia) enabled us to test the modifiability of both implicit and explicit appraisals, and to explore mechanisms of change, which is rather unique. The clinical study on vaginismus was one of the first systematically designed randomized controlled trials.

The results presented in the current thesis provide initial support for the VPI as a device to investigate various determinants of the tolerance and pleasurableness of vaginal pressure as a function of sexual arousal. For this purpose, it is important that the VPI is the first instrument to apply genital pressure while respecting participant's privacy because the device is remotely controlled. The possibility to induce gradually increasing vaginal pressure may reflect the more intense genital stimulation during higher levels of sexual arousal. The suitability of the VPI has been further substantiated by recent studies 189,251 . Our preliminary finding that vaginal pressure may increase sexual arousal (Chapter 5) has been confirmed 189 251. We may conclude that the ecological validity of the measurement of genital sensations as a function of sexual arousal has been successfully improved by the development of the VPI. 
An additional strength of the VPI is that indices of pelvic floor muscles activity can be obtained from the pressure gauge of the VPI (in $\mathrm{mmHg}$ ). These indications of intravaginal pressure, in relation to the activity of the pelvic floor muscles, allows future studies to assess the interplay between emotional states, intravaginal pressure and the tolerance of vaginal pressure.

\section{Clinical implications}

The data presented in the current thesis may help to improve therapeutic outcomes in women with vaginismus and dyspareunia. In the therapist-aided exposure treatment, women were trained in (pelvic) body awareness and relaxation, followed by gradual exposure in vivo via vaginal penetration exercises (with fingers and dilators). These exercises were combined with interventions aiming to cognitively reappraise physical sensations. Three (2-3 hour) exposure sessions were scheduled within one week. Our results showed that eliminating dysfunctional avoidance behaviour by encouraging approach behaviour (exposure) towards feared stimuli is crucial to reduce vaginismus. It is important to explore which specific stimuli are feared, followed by encouraging approach behaviour to reduce distress/anxiety and to maximally violate expectancies 195. Examples of commonly feared stimuli are not having enough space in the vagina, pain and feelings of abnormality. These fears are often triggered by pelvic floor muscle tension, by ripples of the vaginal skinfolds or by feeling the pelvic bone. It is not surprising that these women need a lot of support and instructions to overcome these penetration-related fears during exposure in vivo. An advantage over home-based exposure is that during therapist-aided exposure, the therapist can guide the woman (and her partner) through the difficulty of approaching the penetration-related fears and to manage the associated intense fear, and can encourage non-avoidance. The therapist can assist patients in identifying and verbalizing catastrophic thoughts in order to address them directly.

For clinicians it is relevant to realize that changes in pain catastrophizing cognitions were found to mediate the treatment outcome. These cognitions rapidly changed during the vaginal penetration exercises, and these changes occurred even during the first 
exposure session. This suggests that behavioural experiments are essential to falsify catastrophizing penetration/ pain cognitions in vaginismus. The catastrophic cognitions towards feared stimuli have to be challenged and reframed by new experiences to maximally violate expectancies ${ }^{195}$. To rescript a dysfunctional cognition, it is important to ask what has been learned. This may lead to reformulating a dysfunctional cognition of "inserting a finger will be painful" into one of "When I take more time to relax by focusing on my respiration, it does not hurt".

Changes in positive cognitions were also found to be related to treatment outcome, and positive relabelling of affect and physical sensations may be beneficial in this respect. When inserting fingers into their vagina, women accidently perceive the contact of the fingers with the surrounding tissues as "full" or "no space". They can be encouraged to re-label this cognition into something like "it feels relaxed, warm, soft, open", "it feels like my belly", "the soft/ flexible, adapting tissue of the vagina creates space". An important issue is to learn to differentiate between "painful pressure" and "unknown but normal pressure". Sexual education is important to strengthen positive cognitions. Women and their partners are commonly unfamiliar with the female genitals, in particular with the inner parts of the clitoris and its important function to facilitate sexual arousal and pleasurable intercourse 158,252 . To create a positive perspective, it may help to teach women that sexually functional women, when experiencing explicit sexual stimuli, can experience vaginal pressure as pleasurable and sexually stimulating (Chapter 5).

In view of the high effectivity and the relatively rapid improvements achieved by therapist-aided exposure treatment of vaginismus in the majority of women, clinicians have to question the value of less well-substantiated alternative interventions 57,253 . In home-based exposure, women with vaginismus and their partners are regularly confronted with increasing pain sensations, a panic attack or irrational, intense fears of a finger or tampon remaining "caught" in the vagina. In these highly challenging situations, the assistance of a therapist is crucial for many women to manage these fears and reduce distress by providing concrete instructions and encouraging the women and their partners to continue practicing. When no clear progress is made after three therapeutic sessions of home-based exposure, it is better for couples to be referred to specialized academic hospitals for therapist-aided exposure treatment. Since therapist- 
aided exposure treatment is currently only offered at Leiden and Maastricht, it is desirable to transfer this expertise to other hospitals specializing in genital pain. Noteworthy, a multi-disciplinary team of the Stanford University will start with therapist-aided exposure treatment in vaginismus soon, after they have received a training program of us.

\section{Clinical implications of the VPI}

The development of the VPI has produced an instrument that could be useful for women with vaginismus and dyspareunia. Moreover, we expect that it may also be valuable for women who have suffered sexual abuse, in view of their high levels of fear and avoidance behaviour towards sexual encounters and the sexual problems they have reported 254,255 . In clinical practice, the VPI may be suitable for diagnosis and treatment. The suitability of the VPI for other medical conditions is described in the valorisation chapter of this thesis.

The VPI may be suitable for use in counterconditioning for genital pain, by linking high levels of sexual arousal to gradually increased levels of vaginal pressure, facilitating a positive appraisal of vaginal pressure. Accordingly, a negative valence (genital pain US) of vaginal penetration (CS) could be changed into a positive valence (sexual pleasure US). Evaluative learning (e.g., concerning disgust) has been demonstrated to be sensitive to counterconditioning by pairing the previously opposite valence to the conditioned stimuli 211-214,217. In chronic pain, counterconditioning has been successfully applied by attaching a positive valence to the conditioned stimuli 215,216 . It might be easier to train women to positively reappraise vaginal pressure out of the context that is associated with genital pain cues. This may also help women to experience higher levels of sexual arousal. The VPI may be useful to discover personal preferences regarding pleasurable levels of vaginal pressure and the desired duration of pressure in a sexual and nonsexual context. This may help to identify personal approach-avoidance tendencies. Furthermore, the VPI may help to increase body-awareness and self-efficacy, which may improve treatment outcomes for genital pain $256-258$. Needless to say, future studies will have to explore these application options of the VPI. 


\section{Future directions}

Many interesting issues can be further explored in future studies.

The main challenge for future research is to develop effective interventions that are able to increase sexual pleasure and satisfaction in women suffering from genital pain. Below are some suggested clinical adjustments of the therapist-aided exposure therapy, which need further substantiation. Subsequently, applications of the VPI are proposed to test specific factors related to genital pain and to evaluate specific interventions.

The majority of women with vaginismus showed remarkably rapid improvements in coital frequency, symptoms of vaginismus and coital pain after the therapist-aided exposure treatment. However, $30-40 \%$ of the treated group of women still had elevated levels of fear and pain, and overall, sexual pleasure was not improved, which means there is room for further improvement. Therefore, clinical effect studies are needed to improve the outcome of treatment for genital pain. The proposed adjustments to the therapist-aided exposure treatment follow directly from the studies and the issues discussed above.

It would be valuable to test if the emotion-focused exposure described above could be effective in facilitating sexual pleasure, as well as in reducing fear and avoidance in female genital pain. We expect that the judicious use of safety behaviour in a goalpursuit context, along with emotion regulation skills and flexible goal-directed actions, may be helpful to decrease distress and increase pleasure. In this approach, a detailed and structured assessment of a patient's valences, personal goals and goal-directed actions is used to improve their motivation to engage in and continue treatment 218,259. In the case of female genital pain, this may be important regarding the sexual avoidance goals of vaginal penetration. Accordingly, since pain catastrophizing cognitions were found to mediate therapeutic outcome, it would be valuable to explore if changes in other catastrophizing cognitions with respect to the feared consequences of genital pain (e.g., for the relationship or for sexual self-image) also mediate treatment outcome (see $\left.{ }^{204}\right)$. Indices of treatment outcome probably have to include personal valence-related outcome measures, as well as measures relating to vaginal penetration. A single-case format using daily measurements will be useful for the development of this approach, 
and also to explore mechanisms of change. We expect that both women with dyspareunia and those with vaginismus may benefit from this approach.

As was mentioned above, the VPI provides numerous opportunities for research regarding female genital pain. It would be valuable to experimentally test persistence/avoidance behaviour towards vaginal pressure, as well as the pleasantness or unpleasantness of vaginal pressure as a function of sexual arousal, in both women with dyspareunia and vaginismus. Other interesting topics may be to further explore the extent to which sexual arousal stimuli have the potential to overrule predictors of genital pain, for example, by manipulation of pain/sexual arousal expectations.

It would also be interesting to use the VPI to assess relationships between implicit and explicit concepts of sexual self and appraisals (and changes therein) of vaginal pressure as a function of sexual arousal.

Furthermore, dyadic sex research seems an important avenue for future research, as including both partners in the laboratory setting creates specific relationship dynamics that can yield unique and valid information on sexual responding and the appraisal of vaginal pressure. Recently, valuable insights were obtained by two studies using the VPI to investigate the effects of partner presence, relationship satisfaction, partner congruence, sexual stimulation, and vaginal pressure on the appraisal of vaginal sensations and sexual arousal 189,251. Future studies are needed to better understand the interplay between sexual stimulation and the appraisal of vaginal sensations and the way this perception is shaped by partner and relationship variables, in order to develop specific interventions that target both partners and their relationship.

\section{Conclusions}

This dissertation has presented several studies that aimed to achieve a greater understanding of the mechanisms of genital pain, its treatability and ways to enable genital pleasure. The effectivity of exposure treatment to reduce fear and enable vaginal penetration in women with vaginismus has been further substantiated, and mechanisms of change have been revealed. The development of a new instrument, the VPI, made it 
possible to show for the first time the important role of sexual arousal for a positive appraisal of vaginal pressure. In view of these findings, there is a clear need to know how to enhance positive appraisals and improve sexual arousal in women with genital pain. Further exploration of valences of sex and emotion-focused interventions may be needed. For this purpose, a social and dyadic perspective on genital pain and female sexual pleasure seems indispensable. 



\section{KNOWLEDGE VALORIZATION}

This dissertation focuses on psychological risks and resilience factors related to painful or pleasurable vaginal penetration. In this valorization paragraph I will describe how the obtained knowledge from our research can be made valuable for clinical and social use. I will start with discussing the relevance of the project, by describing the target groups for whom it is relevant and possible activities and products. Subsequently, I will discuss the innovative character of these possible activities and products and how they can be implemented.

\section{Relevance}

Chronic genital pain can be considered as a social problem in view of the high prevalence rates, (5 to $9 \%{ }^{3}$ ), the psychological impact on women and their partners and the barriers for adequate health care. Many women with self-reported genital pain never seek treatment 5 . In the Netherlands, only $37 \%$ of women who had a sexual problem and felt the need to seek help actually contacted a health professional ${ }^{3}$. A number of barriers to seeking help have been reported by women with genital pain including embarrassment and lack of confidence in a medical solution 20.

Genital pain remains one of most poorly understood and complex chronic pain syndromes. Yet little attention is given to this condition and it is frequently dismissed as psychosomatic 260 . Generally, 41\% of women with self-reported genital pain has seen three or more health care providers and only $6 \%$ of women who contacted a clinician obtained advice or help ${ }^{3}$. In specialist care, as many as $15 \%$ of women who seek gynaecological care are affected by genital pain ${ }^{261}$. During gynaecological examination $84 \%$ of affected patients experienced increased pain and around half of the patients with genital pain show high (or very high) levels of palpated vaginal muscular tension ${ }^{25}$. This vaginistic reaction has a negative impact on gynaecological examination or can even preclude it. Women with vaginismus are at increased risk of a delivery by caesarean section, or, when delivering vaginally, to suffer perineal laceration ${ }^{262}$. Consequently, this 
condition carries large costs incurred as a result of both medical treatment and lost productivity.

When confronted with women suffering from genital pain, many health care professionals feel uncomfortable about dealing with pain located in the genital area 8263 . They also feel uncertain about treatment options, and as a result do not engage in a proactive approach ${ }^{21}$. These barriers result in delayed diagnosis and treatment, with deleterious consequences for sexual functioning, well-being, and relationships in women with genital pain ${ }^{19}$.

Thus, genital pain still remains a major health problem in Western countries, leading to significant morbidity and a reduced quality of life for many women ${ }^{19}$. Thus, there is an urgent need for the development of expertise in genital pain. The findings of the studies reported in this thesis contribute to this.

\section{Target groups}

In addition to the academic community, the findings described in this dissertation are relevant for various target groups, namely (1) female genital pain patients and their partners, (2) healthcare providers, (3) sex educators and (4) society.

First and foremost, the results are relevant for genital pain patients and their partners. The experience of genital pain has wide-reaching consequences for affected women and their partners' psychological, sexual, and relationship well-being. The dissertation provides evidence that therapist-aided exposure treatment increases success rates from $19 \%$ to $89 \%$ 59. Therapist-aided exposure is well protocolized and provides practical tools for affected women and their partners. This is important to increasing self-efficacy and providing a positive perspective on treatment outcome in affected couples, which is relevant given their reported lack of confidence in a medical solution.

Affected women may also profit from the Vaginal Pressure Inducer VPI, a new remotely controlled instrument suitable to apply gradually increasing introital vaginal pressure while respecting woman's privacy. The VPI may help to facilitate vaginal penetration and to learn to link vaginal pressure with sexual arousal. In view of the importance of 
sexual arousal to decrease unpleasantness of vaginal pressure (this thesis), this may help to overcome genital pain. To create a positive perspective, it may help to know that sexually functional women can experience vaginal pressure as pleasurable and sexually stimulating, when it is paralleled by explicit sexual stimuli (this thesis).

The second group of stakeholders are healthcare providers. Clinicians may profit from the substantiated relevance of sexual arousal to increase pleasantness of vaginal penetration. They should not limit their focus to reducing genital pain, but should also focus on increasing sexual pleasure. Furthermore, they can focus on targeting avoidance behaviour for changing both negative associations and pain/penetration catastrophizing cognitions in genital pain patients. This knowledge may help to specify clinical interventions. This is important knowledge for sexologists, gynaecologists and pelvic floor therapists. Referring highly anxious genital pain patients for therapist-aided exposure prior to the gynaecological exam or delivery is needed to increase patient friendliness and reduce medical costs. Scheduling combination consults of trained exposure therapists together with health professionals may help to further transfer expertise in clinical practice. For this purpose, training of sexologists, gynaecologists, general practitioners and pelvic floor therapists in therapist-aided exposure is needed in the Netherlands and abroad.

Clinicians may use the VPI in women with genital pain for diagnostic and clinical interventions. The VPI may be suitable for use in counterconditioning for genital pain, by linking high levels of sexual arousal (elicited by explicit film clips) to gradually increasing levels of vaginal pressure, facilitating a positive appraisal of vaginal pressure out of the context that is associated with genital pain cues. The VPI may be useful to identify personal preferences regarding the duration of pressure/penetration along with approach-avoidance tendencies. Furthermore, the VPI may help to increase bodyawareness, self-efficacy and adequate coping, which may improve treatment outcomes for genital pain $256-258$.

The third group of stakeholders are sex educators. They may integrate the finding that explicit sexual arousal stimuli are important to increase pleasurableness of vaginal penetration and prevent pain. In view of the neglected attention for sexual pleasure and 
the related anatomy in women, there is still much room for improvement in sex education.

Fourthly, society can profit from the obtained knowledge. Therapist-aided exposure is not only found to increase effectivity of treatment, also treatment duration can be shortened from ten sessions to three sessions. Furthermore, therapist-aided exposure may facilitate and/or prevent gynaecological interventions. The short duration of therapy increases the suitability to apply this intervention in patients needing gynaecological examination and/or interventions, for example in case of gynaecological diseases or pregnancy in women with increased anxiety. Future studies should test the expected contribution of therapist-aided exposure in medical practice.

\section{Activities and products}

The research reported in the current thesis is part of a project that has resulted in the development of therapist-aided exposure treatment. Following therapist-aided exposure $89 \%$ of the couples was able to have coitus after treatment. These rapidly reached results of therapist-aided exposure can increase the cost-effectiveness of CBT in vaginismus. Therapist-aided exposure is also likely to facilitate as well as prevent gynaecological interventions, along with the costs thereof.

Therapist-aided exposure has been described in several clinical books. Furthermore, the clinical interventions and research are presented to many medical and psychological health professionals on congresses and team visit (sexologists, psychologists, cognitive behavioural therapists and gynaecologists). Furthermore, we have developed a twoweek traineeship in therapist-aided exposure treatment for a multi-disciplinary team in California. The development of therapist-aided exposure treatment and the publications and presentations have led to a critical verbal discussions and opinion papers about the treatment of vaginismus. Nowadays, therapist-aided exposure is commonly accepted and patients coming from all parts of the Netherlands, Belgium, Germany and other countries are referred to our clinics. 
The VPI can be valuable in clinical practice for diagnosis and treatment and can serve as a supplement of/ or an alternative for professional help. When further developed, the VPI may be used as a self-help instrument. Given the amount of women suffering from genital pain and the lack of expert advice or assistance, a trainings program using the VPI may serve as an early-intervention program. This may preclude that acute/ incidental genital pain will turn into a chronic and "neuropathic" disease resulting in huge quantifiable and non-quantifiable costs ${ }^{264}$. This may lead to improvements in the psychosocial well-being of genital pain patients and their partners, which may also serve an economical interest, as it might help in reducing the direct and indirect costs associated with chronic genital pain.

The VPI may also be valuable for women who have suffered sexual abuse, in view of their high levels of fear and avoidance behaviour towards sexual encounters and the sexual problems they experience 254,255 . The VPI may help to encourage approach behaviour towards genital stimuli in women with negative experiences 265 .

Furthermore, the VPI may be applicable in several groups of patients with medical diseases to facilitate or enable vaginal penetration: patients with diseases like MRKH (Maier Rokitanski Kustner Syndrome or vaginal agenesis)); patients with a physical constriction of the vagina caused by lichen sclerosis 266,267 ) male to female gender confirmation surgeries, infections, vaginal narrowing/ shortening (side effect of cancer treatment, surgeries, genetic defects), injuries and auto-immune conditions (Graph vs. host diseases). In view of maintaining the accessibility of the vagina, these patients have to apply vaginal dilatation exercises. These exercises are often experienced as technical and carrying, especially because they have to start immediately after operation. Vaginal dilation using the VPI may increase patient friendliness. The combination of sexual arousal and vaginal dilation facilitates dilatation and may improve mucosal color, moisture and vaginal elasticity and decreased bleeding and ulceration in irradiated cervical cancer patients ${ }^{268}$. With some adaptations the VPI could be easily inserted at night whereby the balloon of the VPI gradually extends several times at the night, preferably during the rapid eye movement (REM) sleep because pain sensitivity is lower then. This may also be useful for women with a partner having a large penis. 
Finally, the VPI can be used as a pleasure instrument, as the vaginal pressure applied by the VPI is found to increase sexual arousal in a sexual context (this thesis). Physically disabled patients have already shown interest in order to experience sexuality in privacy. This might also be interestingly for the majority of women (70\%) who is not used to get an orgasm during intercourse ${ }^{269}$. When vaginal pressure may increase sexual arousal, this may also prevent genital pain.

The VPI is suitable and available for future studies. In cooperation with two Swedish universities we try to improve this device by making it adaptive, i.e., able to exert dynamically different pressures on the vaginal duct to simultaneously guarantee comfort levels and achieve the medical dilation objectives. Another aim is to implement feedback control connecting the patients' comfort levels with their experienced physiological stimuli in a following version of the VPI. Furthermore, we try to facilitate and improve the usability of the VPI by the use of synthetic materials to replace the balloon. The possibilities to further develop the VPI will depend on the financial resources and the involvement of a company. In cooperation with Biomed booster a procedure has started to obtain a patent of the European Patent Office, in order to investigate the commercial potential of the VPI. In time it became clear that the chances for a commercial exploitation of the device are restricted, and thus the procedure was terminated.

Noteworthy, the first publication of the VPI elicited much attention on social media. Some provided adequate information, based on the published article:

https://www.eoswetenschap.eu/psyche-brein/de-voordelen-van-voorspel. https://www.medicalfacts.nl/2017/11/27/tool-om-pijn-bij-geslachtsgemeenschap-temeten/.

One journalist from IB times interviewed me and published relevant information, but unfortunately add a juicy incorrect title/ interpretation:

https://www.ibtimes.co.uk/scientists-just-accidentally-invented-entirely-new-kind-sextoy-women-1630404.

Other sides published incorrect information about the study. Some examples are: 
https://punchng.com/scientists-accidentally-invent-new-sex-toy-for-women/

https://www.dailymail.co.uk/health/article-4717142/Want-orgasm-Try-inflatable-sex$\underline{\text { toy.html }}$

Journalists from these sides never had any contact with the researchers; they just based the information on the published article - adding incorrect details,- or copied information from other sides, - with more exaggeration as a result.

Scientists better consider in advance whether they would take the risk to accept an interview with a journalist from a social side, even though the quality of that specific side seems acceptable. However, we think it is hard and probably impossible to control the social media in research on juicy stuff... they just take the information from scientific journals and ran with it. Despite that, it can still help to inform the public, break the taboo. We have experienced that, the storm on social media was over quickly and had little impact.

\section{Innovation}

A problem in dealing with vaginism is due to the fact that vaginism is a physical response to fear. On the one hand, physicians and pelvic floor therapists taught patients how to tighten and relax the pelvic floor muscles, but hey were not trained in coping with anxiety/ highly anxious patients. On the other hand, psychologists applied CBT using self-exposure (exposure in vivo without assistance of the therapist), but they lacked knowledge of the physical anatomy and specific feared physical triggers and responses to fear. The differentiation between a physical and psychological approach did not help the highly anxious patients to enable and execute vaginal penetration exercises. The first innovation of therapist-supported exposure was to allow psychologists to apply this physical approach in which women were aided with practising vaginal dilation exercises. We started to execute this exposure together with several physicians. This has resulted in the development of the expertise needing for exposure, like coping with specific feared stimuli, hypervigilance, panic attacks, blood phobia as well as various physical and psychological exercises/ advices to enable vaginal 
penetration and violate expectancies. This has resulted in a highly specialised, innovative and effective treatment of vaginismus.

The development of a new instrument was needed because no instrument was available to apply standardized introital vaginal pressure in a laboratory while respecting the privacy of participants. The VPI is the first instrument to induce gradually increasing vaginal pressure in a standardized and controlled manner in the introitus vaginae. The possibility to induce gradually increasing vaginal pressure may reflect the more intense genital stimulation during higher levels of sexual arousal. Furthermore, the VPI is the first instrument to apply genital pressure while respecting participant's privacy because the device is remotely controlled. This helps to guarantee a sexual context and to match the experimental setting as closely as possible to the context in which these sensations naturally occur. The VPI has made it possible to substantiate for the first time, the important role of sexual arousal for a positive appraisal of vaginal pressure.

\section{Schedule and implementation}

In order to facilitate the process of implementation of the developed expertise to clinical practice, several endeavors have been taken. Together with an international group of experts on vaginismus, we have published a commentary on DSM-5 with respect to The Demise of Vaginismus in Favor of Genito-Pelvic Pain/Penetration Disorder in a renowned international journal (Archives of Sexual behaviour). Several newspapers, magazines and local TV channels have payed attention to both the therapist-aided exposure and the VPI. Several social media have paid much attention to the VPI. The expertise towards therapist-aided exposure is described in several national and international book chapters for clinicians and is available via a Dutch website on sexual dysfunctions (www.seksueledisfuncties.nl). The final chapter of this dissertation is submitted and is expected to be accessible within a year. Furthermore, the studies and clinical interventions are presented to many medical and psychological health professionals on congresses and team visit (sexologists, psychologists, cognitive behavioural therapists and gynaecologists). The findings with respect to the VPI are presented to physically disabled patients and their health care takers. 
Stanford Medical Center of California has recently started with the first therapist-aided exposure under our supervision. After the permission of the Board of Directors of Stanford Medical Center of California, depending on our endorsement, our colleagues are intended to set up a private clinic for therapist-aided exposure treatment in California, aiming to generate finances for less fortunate patients to get this treatment in Stanford Medical Center.

Strikingly, in the Netherlands, therapist-aided exposure is not yet adopted by colleagues from other hospitals. With respect to patient friendliness and a further development of expertise, it is undesirable that this specialized therapy is only available in the academic hospitals Leiden and Maastricht. In the academic hospital in Leiden there are 5 sexologists involved in this treatment, in Maastricht there is only one sexologist applying therapist-aided exposure (the author of this thesis), which is a severe vulnerable and irresponsible situation. There are several obstacles to the transfer of this expertise in the Netherlands. Firstly, we are intended to set up a following study on vaginismus aiming to reduce post-treatment genital pain. Because women with primary vaginismus are difficult to recruit, it is undesirable to distribute patients to various institutions. Secondly, in the academic field, the development of new studies, expertise and publications seems to be valued more than the implementation of developed knowledge. In view of the restricted involved specialists, together with the various tasks related to patient care, education and research, also a lack of time is playing a role. Thirdly, international publications, cooperation and transfer of expertise are of higher value than national activities. This may lead to the bizarre situation that foreign colleagues might be better informed about new developments than our own colleagues in the Netherlands. Fourthly, many colleagues feel reserved towards starting with this as complicated experienced, specialised treatment. Fifthly, the introduction of market forces in health care strengthens financial motives and increases competition. Here, offering highly specialized care and maintaining exclusivity conflicts with the value of a transfer of expertise.

Solutions for these problems hindering the transferring of knowledge are not easily found. Unfortunately, a policy conversation with health insurance companies to enable a financial compensation for this highly specialized treatment in vaginismus in the specialized Mental Health Care (gespecialiseerde GGZ) did not have any result. 
Consequently, the survival of this care depends on the local management of the hospitals. To implement this expertise in different regions in the Netherlands, it may help to offer a training program on the application of therapist-guided exposure treatment, with the primary aim of setting up a national multidisciplinary team of specialists in the Netherlands, followed by the permission from local policy makers. This may generate intervision possibilities, future research and the development of expertise.

Finally, the results of this dissertation have contributed to new research and projects. Firstly, we are intending to assess the effect of a modified version of therapist-aided exposure treatment on women with dyspareunia and/ or sexual abuse experiences. The first clinical experiences are promising. To set up this project, a brainstorm meeting with experts on anxiety of the Academic Center of Anxiety (Mondriaan Zorg Groep), University Maastricht and the Maastricht UMC will be scheduled this year. Possibly, the use of the VPI for diagnostic and clinical applicability's, e.g. to apply exposure and/or EMDR on women with sexual abuse experiences and/ or genital pain, can be integrated in this project. With respect to the finances, we are intended to write a business case to the academic hospital to create opportunities to further integrate and develop this therapy.

Secondly, there are plans to develop an online trainings/ educational program to share our expertise for women with genital pain, in cooperation with Brightness Maastricht Health Campus. For this, it is needed to assess the effect of sexual arousal on the appraisal of vaginal pressure in women with genital pain by use of the VPI. Together with this, the training effect of the VPI on the genital pain can be explored. A final aim is to find grants and/or to start up a company to further realise a more user-friendly version of the VPI. This product can be merchandised in combination with an online prevention/ self-help program in order to prevent and overcome genital pain. 



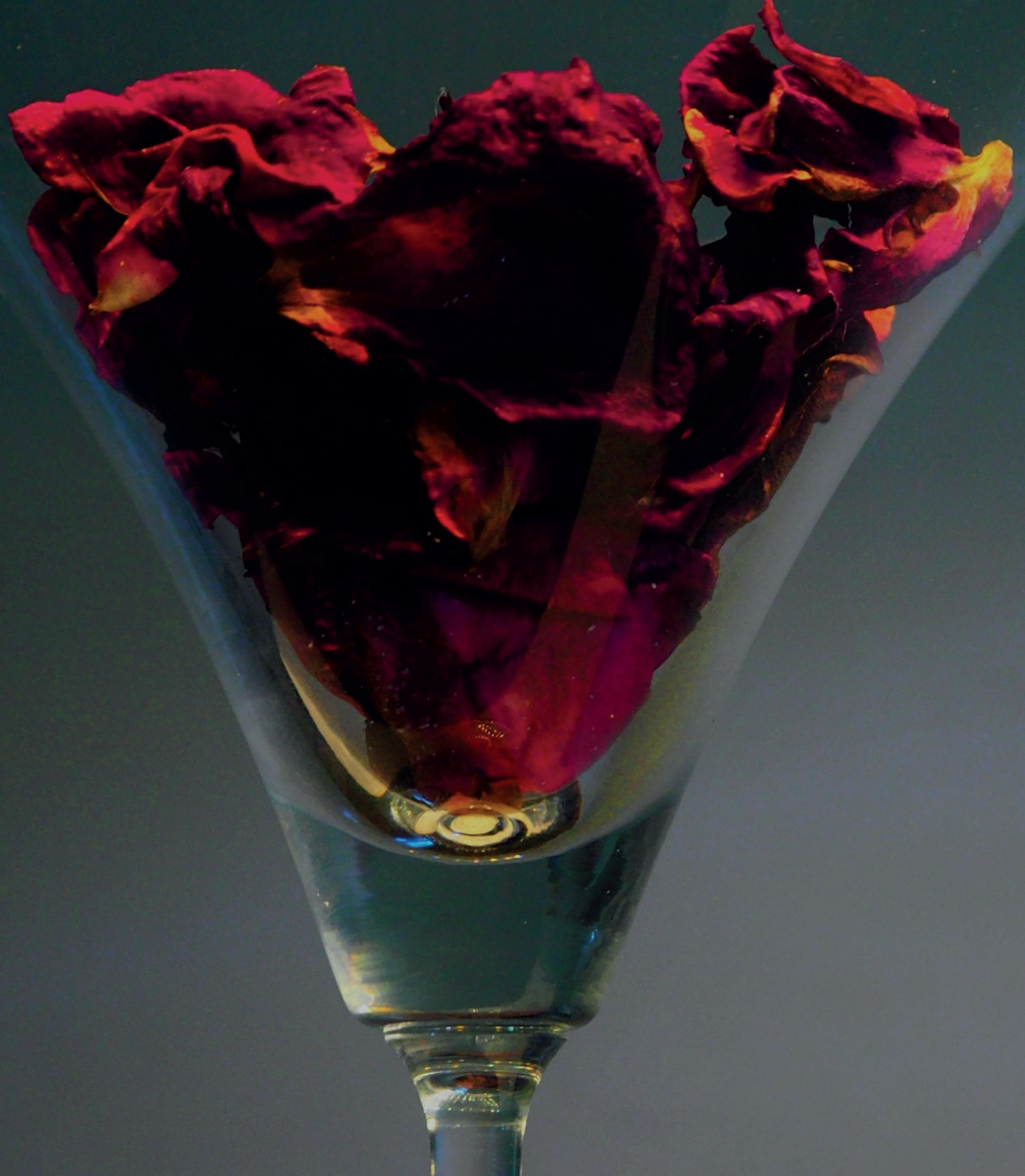

Samenvatting 
De onderzoeken die in dit proefschrift worden gerapporteerd hadden als doel om zowel psychologische risicofactoren als beschermende factoren te exploreren die samenhangen met pijnlijke en/of plezierige vaginale penetratie.

Uitgaande van het cognitieve verwerkingsmodel (het informatie proces-model) van seksuele opwinding en de vicieuze cirkel van genitale pijn (CGT model) hebben we gekeken of aandachtsbias (afleiding) in verband kan worden gebracht met genitale pijn. Daarnaast hebben we onderzocht of negatieve associaties samenhangen met genitale pijn. Hierbij hebben we zowel impliciete (automatische) associaties, als expliciete (subjectieve) associaties geanalyseerd. Verder hebben we bekeken of seksuele opwinding van belang is voor een positieve waardering van vaginale druk. We hebben vijf experimentele studies uitgevoerd. In deze paragraaf geven we een Nederlandse samenvatting van deze studies.

In de eerste studie (Hoofdstuk 2) hebben we bekeken of meer aandacht voor seksgerelateerde dreiging (o.a. pijn) en minder positieve impliciete associaties met seksuele stimuli samenhangen met minder seksuele opwinding en meer genitale pijn. In deze studie hebben we associaties van seks met dreiging en van seks met motivatie getest bij vrouwen met genitale pijn en vrouwen zonder seksuele klachten. Een groep vrouwen met genitale pijn heeft de neiging de gemeenschap te vermijden (vrouwen met vaginisme), terwijl een andere groep juist de neiging heeft te persisteren in de pijnlijke gemeenschap (vrouwen met dyspareunie). We wilden onderzoeken of deze verschillen in het vermijden van/ persisteren in de penetratie samenhangen met verschillen in aandacht (voor seks/ pijn) en/of associaties met seks (meer dreiging en minder motivatie). Hiertoe hebben we vrouwen met primair (levenslang) vaginisme vergeleken met vrouwen met dyspareunie en seksueel functionerende vrouwen. Om aandachtsbias voor pijn/ seks te testen hebben we een "Visual search Task" (een visuele zoek taak, VST) gebruikt. Hierbij hebben we zowel detectie (vigilantie) van seks- en pijnstimuli onderzocht, als aandachtsafleiding (distraction). Associaties zijn getest met een "singletarget Implicit Association Test" (enkelvoudige associatie test, st-IAT). We gebruikten twee versies van de stIAT om associaties van seks met dreiging, en associaties van seks met motivatie te testen. 
We vonden geen verschillen tussen de groepen in aandachtsbias, noch in impliciete associaties. Daarnaast toonden correlationele analyses aan dat een vertraagde detectie van seksuele stimuli en impliciete associaties van seks met dreiging, samenhangen met minder seksuele opwinding in het dagelijks leven. Dit gold voor vrouwen met en zonder genitale pijn.

Deze bevindingen komen niet overeen met de suggestie dat aandachtsbias voor pijn of seks bijdraagt aan genitale pijn, of dat verschillen in het vermijden van penetratie verklaard kunnen worden door verschillen in aandachtsbias of impliciete associaties met dreiging of motivatie. Echter, de correlationele bevindingen onderbouwen de veronderstelling dat dreigende impliciete associaties en verminderde aandacht voor seksuele stimuli kunnen interfereren met het genereren en het verloop van seksuele opwinding.

In de volgende studie (Hoofdstuk 3) hebben we de rol van negatieve globale affectieve associaties met erotische stimuli bij vaginisme geëxploreerd. Hiertoe hebben we de globale affectieve associaties met erotische en vaginale penetratiestimuli getest in vrouwen met vaginisme en een controlegroep. Daarnaast hebben we de impliciete en expliciete dreigende associaties met seksuele penetratie stimuli onderzocht. Hiervoor gebruikten we een st-IAT, die vergelijkbaar was met de eerder gebruikte st-IAT in hoofdstuk 2. Globale impliciete en expliciete affectieve associaties onderzochten we met een "Affective Simon Task” (AST).

De resultaten toonden aan dat vrouwen met vaginisme, vergeleken met controle vrouwen, op expliciet niveau relatief dreigende associaties hadden en minder positieve globale affectieve associaties met seksuele stimuli. We vonden geen verschillen tussen de groepen in impliciete associaties. Deze bevindingen suggereren dat dreigende en negatieve globale affectieve associaties op expliciet niveau beide een rol spelen bij vaginisme. De studies die beschreven zijn in hoofdstuk 2 and 3 toonden niet aan dat vrouwen met genitale pijn op impliciet niveau relatief negatieve associaties (dreiging, motivatie of global affect) met seks hebben.

In de studie in hoofdstuk 3 hebben we tevens onderzocht of bij primair vaginisme, impliciete en expliciete dreigende en relatief negatieve globaal affectieve associaties met erotische stimuli kunnen veranderen door begeleide exposure (Hoofdstuk 3). Resultaten 
lieten zien dat begeleide exposure effectief was in het verminderen van expliciet dreigende associaties en een positief effect had op de expliciete globaal affectieve associaties met seksuele stimuli; de impliciete associations bleven onveranderd.

In Hoofdstuk 4 hebben we een studie beschreven waarin we veranderingsmechanismen hebben onderzocht van begeleide exposure bij vrouwen met vaginisme. We vonden dat de uitkomst van behandeling (frequentie van coitus, symptomen van vaginisme en genitale pijn) tijdens 12 weken gemedieerd werd door veranderingen in negatieve en positieve penetratiecognities tijdens 6 weken, hetgeen voornamelijk beinvloed werd door een sterkere vermindering van catastroferende pijn/ penetratiecognities.

In de laatste twee studies (Hoofdstuk 5 en Hoofdstuk 6) wilden we de veronderstelde positieve invloed van seksuele opwinding op het voorkomen en overwinnen van genitale pijn onderzoeken. Voor dit doel is een nieuw instrument ontwikkeld, de "Vaginal Pressure Inducer" (vaginale druk instrument, VPI). Met behulp van de VPI trachtten we de vaginale sensaties tijdens penetratie te simuleren. Tevens wilden we de experimentele setting zoveel mogelijk laten lijken op de context waarin deze sensaties voorkomen.

De studie die beschreven is in Hoofdstuk $\mathbf{5}$ had als doel om de invloed van seksuele opwinding op de ervaring van vaginale druksensaties beter te begrijpen bij een groep seksueel functionerende vrouwen. Hiertoe induceerden we, met behulp van de VPI, geleidelijk toenemende vaginale druk in de introïtus van de vagina in een neutrale, erotische en een expliciet seksuele conditie.

De resultaten lieten zien dat de drempel van onaangename vaginale druk werd verhoogd in een seksuele conditie, vergeleken met een niet-seksuele conditie. Bovendien werd de vaginale druk als plezieriger ervaren in de seksuele conditie, en met name als vrouwen naar de expliciete seks film keken. Tevens suggereerden de bevindingen dat vaginale druk seksueel opwindend kan zijn voor seksueel functionerende vrouwen; Vrouwen vonden de seks films opwindender als deze gecombineerd werden met vaginale druk. Op basis van de uitkomst van de eerste studie lijkt de VPI een geschikt instrument om verschillende determinanten van een aangename en onaangename waardering van vaginale druk te onderzoeken. 
In onze laatste studie (Hoofdstuk 6) hebben we voorspellers onderzocht voor de waardering van de, met de VPI geinduceerde, vaginale druk. Hiertoe werd bij de onderzochte groep vrouwen van de vorige studie de eerste acclimatiesatie conditie m.b.t. de vaginale druk vergeleken met een volgende seksuele en niet-seksuele conditie. We onderzochten in hoeverre individuele verschillen (verhoogde angstgevoeligheid, pijnvigilantie en verminderde seksuele opwinding in het dagelijks leven) samenhingen met de waardering van vaginale druk in een seksuele en niet-seksuele context. Verder exploreerden we de mediërende rol van verwachtingen (pijn en plezier) van vaginale druk.

In overeenstemming met onze verwachtingen, bevestigden de resultaten de voorspellende rol van verhoogde angstgevoeligheid, pijnvigilantie en verminderde seksuele opwinding voor pijnlijke druk-sensaties; echter, dit gold alleen voor de acclimatisatieconditie (dus bij de eerste ervaring met de vaginale druk, zonder seksuele stimulatie). Pijnverwachtingen mediëerden de relatie tussen angst en pijnlijke vaginale druk alsmede de relatie tussen pijn vigilantie en pijnlijke vaginale druk. In de overige condities werden significante en positieve associaties gevonden tussen pijnverwachtingen en pijnlijke vaginale druk in een niet-seksuele context, en tussen seksuele opwinding in het dagelijks leven en plezierige vaginale druk in een seksuele context. We concludeerden dat het verminderen van pijnverwachtingen en versterken van seksuele opwinding belangrijke doelen zijn van behandeling om genitale pijn te voorkomen of te overwinnen.

\section{Conclusies}

Als we de resultaten evalueren die in dit proefschrift zijn gepresenteerd aangaande de, in Hoofdstuk 1 geformuleerde, belangrijkste onderzoeksvraag: Welke impliciete en expliciete cognitieve processen liggen ten grondslag aan pijnlijke of plezierige vaginale penetratie bij vrouwen?, kunnen de volgende antwoorden worden gegeven:

De bevindingen van onze studies suggereren dat de betrokkenheid van negatieve associaties met seks bij genitale pijn bij vrouwen beperkt is tot een expliciet niveau (Chapter 2 en 3). De versterkte expliciete dreiging en negatieve globaal affectieve 
associaties met seksuele stimuli werden succesvol veranderd door begeleide exposure bij vrouwen met vaginisme (Hoofdstuk 3). De meeste koppels (90\%) waren in staat tot geslachtsgemeenschap na behandeling, en de uitkomst (in termen van frequentie van coitus, symptomen van vaginisme en pijn bij gemeenschap) werd gemedieerd door een versterkte reductie van catastroferende pijncognities (Hoofdstuk 4). Het belang van een sterke seksuele opwinding voor een positieve waardering van vaginale druk is wetenschappelijk onderbouwd. Pijnlijke vaginale druk werd beinvloed door individuele verschillen, in het bijzonder pijnverwachtingen, maar alleen in de afwezigheid van seksuele stimuli. Vrouwen met meer ervaringen met (sterke) seksuele opwinding associeerden vaginale druk met meer plezier. 


\section{References}

1 Janssen, E., Everaerd, W., Spiering, M. \& Janssen, J. Automatic Processes and the Appraisal of Sexual stimuli: Toward an Information Processing Model of Sexual Arousal. J. Sex Res. 37, 8-23 (2000).

ter Kuile, M. M., Both, S. \& van Lankveld, J. Cognitive behavioral therapy for sexual dysfunctions in women. Psychiatr. Clin. North Am. 33, 595-610 (2010).

3 de Graaf, H. \& Wijsen, C. Seksuele gezondheid in Nederland, Delft, Eburon. (2017).

4 Arnold, L. D., Bachmann, G. A., Rosen, R. \& Rhoads, G. G. Assessment of vulvodynia symptoms in a sample of US women: a prevalence survey with a nested case control study. Am. J. Obstet. Gynecol. 196, 128 e121-126, doi:10.1016/j.ajog.2006.07.047 (2007).

Harlow, B. L. et al. Prevalence of symptoms consistent with a diagnosis of vulvodynia: population-based estimates from 2 geographic regions. Am. J. Obstet. Gynecol. 210, 40 e41-48, doi:10.1016/j.ajog.2013.09.033 (2014).

6 Reed, B. D. et al. Prevalence and demographic characteristics of vulvodynia in a population-based sample. Am. J. Obstet. Gynecol. 206, 170 e171-179, doi:10.1016/j.ajog.2011.08.012 (2012).

Danielsson, I., Sjoberg, I., Stenlund, H. \& Wikman, M. Prevalence and incidence of prolonged and severe dyspareunia in women: results from a population study. Scand J Public Health 31, 113-118 (2003).

Dewitte, M., Borg, C. \& Lowenstein, L. A psychosocial approach to female genital pain. Nature reviews. Urology 15, 25-41, doi:10.1038/nrurol.2017.187 (2018).

9 American Psychiatric Association. Diagnostic and Statistical Manual of Mental Disorders. Vol. Fifth Edition (American Psychiatric Publishing, 2013).

American Psychiatric Association. Diagnostic and Statistical Manual of Mental Disorders. Vol. Fourth edition, and Text revision (DC, 2000).

van Lankveld, J. J. et al. Women's sexual pain disorders. J Sex Med 7, 615-631 (2010).

Meston, C. M. \& Bradford, A. Sexual dysfunctions in women. Annual review of clinical psychology 3, 233-256, doi:10.1146/annurev.clinpsy.3.022806.091507 (2007).

13 Reissing, E. D., Binik, Y. M., Khalife, S., Cohen, D. \& Amsel, R. Vaginal spasm, pain, and behavior: an empirical investigation of the diagnosis of vaginismus. Arch. Sex. Behav. 33, 5-17, doi:10.1023/B:ASEB.0000007458.32852.c8 (2004a).

Reissing, E. D., Brown, C., Lord, M. J., Binik, Y. M. \& Khalife, S. Pelvic floor muscle functioning in women with vulvar vestibulitis syndrome. J. Psychosom. Obstet. Gynaecol. 26, 107-113 (2005).

15 Basson, R. et al. Definitions of women's sexual dysfunction reconsidered: advocating expansion and revision. J. Psychosom. Obstet. Gynaecol. 24, 221-229 (2003).

Lewis, R. W. et al. Epidemiology/risk factors of sexual dysfunction. Journal of Sexual Medicine 1, 35-39, doi:10.1111/j.1743-6109.2004.10106.x (2004).

ter Kuile, M. M., Van Lankveld, J. J., Vlieland, C. V., Willekes, C. \& Weijenborg, P. T. Vulvar vestibulitis syndrome: an important factor in the evaluation of lifelong vaginismus? J. Psychosom. Obstet. Gynaecol. 26, 245-249 (2005).

Lahaie, M. A. et al. Can Fear, Pain, and Muscle Tension Discriminate Vaginismus from Dyspareunia/Provoked Vestibulodynia? Implications for the New DSM-5 
Diagnosis of Genito-Pelvic Pain/Penetration Disorder. Arch. Sex. Behav. 44, 1537-1550, doi:10.1007/s10508-014-0430-z (2015).

19 Bergeron, S., Corsini-Munt, S., Aerts, L., Rancourt, K. \& Rosen, N. O. Female Sexual Pain Disorders: a Review of the Literature on Etiology and Treatment. Current sexual health reports, doi:DOI 10.1007/s11930-015-0053-y (2015).

20 Donaldson, R. L. \& Meana, M. Early dyspareunia experience in young women: confusion, consequences, and help-seeking barriers. J Sex Med 8, 814-823, doi:10.1111/j.1743-6109.2010.02150.x (2011).

21 Hinchliff, S. \& Gott, M. Seeking medical help for sexual concerns in mid- and later life: a review of the literature. J. Sex Res. 48, 106-117, doi:10.1080/00224499.2010.548610 (2011).

22 Meana, M., Binik, Y. M., Khalife, S. \& Cohen, D. R. Biopsychosocial profile of women with dyspareunia. Obstet. Gynecol. 90, 583-589. (1997).

23 van der Velde, J. \& Everaerd, W. The relationship between involuntary pelvic floor muscle activity, muscle awareness and experienced threat in women with and without vaginismus. Behav. Res. Ther. 39, 395-408 (2001).

24 van der Velde, J., Laan, E. \& Everaerd, W. Vaginismus, a component of a general defensive reaction. an investigation of pelvic floor muscle activity during exposure to emotion-inducing film excerpts in women with and without vaginismus. Int. Urogynecol. J. Pelvic Floor Dysfunct. 12, 328-331 (2001).

25 de Kruiff, M. E., ter Kuile, M. M., Weijenborg, P. T. \& van Lankveld, J. J. Vaginismus and dyspareunia: is there a difference in clinical presentation? J. Psychosom. Obstet. Gynaecol. 21, 149-155 (2000).

26 Brinkman. Langdurige gedragstherapie in een geval van vaginisme. Tijdschrift voor gedragstherapie 1, 54-59. (1975).

27 Bezemer, W. in Handboek seksuele hulpverlening Vol. II Handboek seksuele hulpverlening (eds M.C.T. Moors-Mommers et al.) Ch. II.B.2.4., II.B.2.4.Bez.1Bez. 24 (Van Loghum Slaterus, 1988).

28 Leclerc, B., Bergeron, S., Binik, Y. M. \& Khalife, S. History of sexual and physical abuse in women with dyspareunia: association with pain, psychosocial adjustment, and sexual functioning. J Sex Med 7, 971-980, doi:10.1111/j.17436109.2009.01581.x (2010).

29 Rosen, N. O., Sadikaj, G. \& Bergeron, S. Within-person variability in relationship satisfaction moderates partners' pain estimation in vulvodynia couples. Pain 156, 703-710, doi:10.1097/j.pain.0000000000000101 (2015).

30 Rosen, N. O., Bergeron, S., Leclerc, B., Lambert, B. \& Steben, M. Woman and partnerperceived partner responses predict pain and sexual satisfaction in provoked vestibulodynia (PVD) couples. J Sex Med 7, 3715-3724, doi:10.1111/j.17436109.2010.01957.x (2010).

31 Pazmany, E., Bergeron, S., Verhaeghe, J., Van Oudenhove, L. \& Enzlin, P. Sexual communication, dyadic adjustment, and psychosexual well-being in premenopausal women with self-reported dyspareunia and their partners: a controlled study. J Sex Med 11, 1786-1797, doi:10.1111/jsm.12518 (2014).

32 Leclerc, B. et al. Attachment, Sexual Assertiveness, and Sexual Outcomes in Women with Provoked Vestibulodynia and Their Partners: A Mediation Model. Arch. Sex. Behav. 44, 1561-1572, doi:10.1007/s10508-014-0295-1 (2015).

33 Spano, L. \& Lamont, J. Dyspareunia: a symptom of female sexual dysfunction. Can. Nurse 71, 22-25 (1975). 
34 Vlaeyen, J. W. \& Linton, S. J. Fear-avoidance and its consequences in chronic musculoskeletal pain: a state of the art. Pain 85, 317-332 (2000).

Klaassen, M. \& Ter Kuile, M. M. Development and initial validation of the vaginal penetration cognition questionnaire (VPCQ) in a sample of women with vaginismus and dyspareunia. J Sex Med 6, 1617-1627 (2009).

36 Both, S., Brauer, M., Weijenborg, P. \& Laan, E. Effects of Aversive Classical Conditioning on Sexual Response in Women With Dyspareunia and Sexually Functional Controls. J Sex Med 14, 687-701, doi:10.1016/j.jsxm.2017.03.244 (2017).

Cherner, R. A. \& Reissing, E. D. A Comparative Study of Sexual Function, Behavior, and Cognitions of Women with Lifelong Vaginismus. Arch. Sex. Behav., doi:10.1007/s10508-013-0111-3 (2013).

Desrochers, G., Bergeron, S., Landry, T. \& Jodoin, M. Do psychosexual factors play a role in the etiology of provoked vestibulodynia? A critical review. J. Sex Marital Ther. 34, 198-226, doi:10.1080/00926230701866083 (2008).

39 Brauer, M., de Jong, P. J., Huijding, J., Laan, E. \& ter Kuile, M. M. Automatic and deliberate affective associations with sexual stimuli in women with superficial dyspareunia. Arch. Sex. Behav. 38, 486-497, doi:10.1007/s10508-008-9367-4 (2009).

Borg, C., de Jong, P. J. \& Schultz, W. W. Vaginismus and dyspareunia: automatic vs. deliberate disgust responsivity. J Sex Med 7, 2149-2157, doi:10.1111/j.17436109.2010.01800.x (2010).

41 Huijding, J., Borg, C., Weijmar-Schultz, W. \& De Jong, P. J. Automatic Affective Appraisal of Sexual Penetration Stimuli in Women with Vaginismus or Dyspareunia. J Sex Med 8, 806-813 (2011).

Desrochers, G., Bergeron, S., Khalife, S., Dupuis, M. J. \& Jodoin, M. Fear avoidance and self-efficacy in relation to pain and sexual impairment in women with provoked vestibulodynia. Clin. J. Pain 25, 520-527, doi:10.1097/AJP.0b013e31819976e3 (2009).

43 Pukall, C. F., Binik, Y. M., Khalife, S., Amsel, R. \& Abbott, F. V. Vestibular tactile and pain thresholds in women with vulvar vestibulitis syndrome. Pain 96, 163-175 (2002).

44 Jodoin, M. et al. Attributions about pain as predictors of psychological symptomatology, sexual function, and dyadic adjustment in women with vestibulodynia. Arch. Sex. Behav. 40, 87-97, doi:10.1007/s10508-010-9647-7 (2011).

45 Thomten, J. \& Linton, S. J. A psychological view of sexual pain among women: applying the fear-avoidance model. Womens Health (Lond Engl) 9, 251-263, doi:10.2217/whe.13.19 (2013).

Brauer, M., Laan, E. \& ter Kuile, M. M. Sexual arousal in women with superficial dyspareunia. Arch. Sex. Behav. 35, 191-200, doi:10.1007/s10508-005-9001-7 (2006).

Wouda, J. C. et al. Vaginal plethysmography in women with dyspareunia. J. Sex Res. 35, 141-147 (1998).

48 Shafik, A. \& El Sibai, O. Study of the pelvic floor muscles in vaginismus: a concept of pathogenesis. Eur. J. Obstet. Gynecol. Reprod. Biol. 105, 67-70 (2002).

49 Reissing, E. D. Re-evaluating vaginismus: An empirical investigation of diagnostic reliability, vaginal spasm, pain, and associated etiological correlates. 
Dissertation Abstracts International: Section B: The Sciences and Engineering 64, 5799 (2004b).

50 Sutherland, O. Qualitative analysis of heterosexual women's experience of sexual pain and discomfort. J. Sex Marital Ther. 38, 223-244, doi:10.1080/0092623X.2011.606880 (2012).

51 Flink, I. K., Engman, L., Ter Kuile, M. M., Thomten, J. \& Linton, S. J. Coping with pain in intimate situations: Applying the avoidance-endurance model to women with vulvovaginal pain. Scandinavian Journal of Pain 17, 302-308, doi:10.1016/j.sjpain.2017.08.007 (2017).

52 Brauer, M., Lakeman, M., van Lunsen, R. \& Laan, E. Predictors of task-persistent and fear-avoiding behaviors in women with sexual pain disorders. J Sex Med 11, 3051-3063, doi:10.1111/jsm.12697 (2014).

53 Borg, C., Peters, M. L., Schultz, W. W. \& de Jong, P. J. Vaginismus: heightened harm avoidance and pain catastrophizing cognitions. J Sex Med 9, 558-567, doi:10.1111/j.1743-6109.2011.02535.x (2012).

54 Payne, K. A. et al. Effects of sexual arousal on genital and non-genital sensation: a comparison of women with vulvar vestibulitis syndrome and healthy controls. Arch. Sex. Behav. 36, 289-300, doi:10.1007/s10508-006-9089-4 (2007).

55 Bergeron, S. et al. A randomized comparison of group cognitive--behavioral therapy, surface electromyographic biofeedback, and vestibulectomy in the treatment of dyspareunia resulting from vulvar vestibulitis. Pain 91, 297-306 (2001).

56 Brotto, L. A., Yong, P., Smith, K. B. \& Sadownik, L. A. Impact of a multidisciplinary vulvodynia program on sexual functioning and dyspareunia. J Sex Med 12, 238247, doi:10.1111/jsm.12718 (2015).

57 van Lankveld, J. J. et al. Cognitive-behavioral therapy for women with lifelong vaginismus: a randomized waiting-list controlled trial of efficacy. J. Consult. Clin. Psychol. 74, 168-178 (2006).

58 Ter Kuile, M. M. et al. Therapist-aided exposure for women with lifelong vaginismus: a replicated single-case design. J. Consult. Clin. Psychol. 77, 149159, doi:10.1037/a0014273 (2009).

59 Ter Kuile, M. M., Melles, R., de Groot, H. E., Tuijnman-Raasveld, C. C. \& van Lankveld, J. Therapist-aided exposure for women with lifelong vaginismus: a randomized waiting-list control trial of efficacy. J. Consult. Clin. Psychol. 81, 1127-1136, doi:10.1037/a0034292 (2013).

60 Brauer, M., ter Kuile, M. M., Janssen, S. A. \& Laan, E. The effect of pain-related fear on sexual arousal in women with superficial dyspareunia. Eur J Pain 11, 788798 (2007).

61 Both, S., van Lunsen, R., Weijenborg, P. \& Laan, E. A new device for simultaneous measurement of pelvic floor muscle activity and vaginal blood flow: a test in a nonclinical sample. J Sex Med 9, 2888-2902, doi:10.1111/j.17436109.2012.02910.x (2012).

62 Blok, B. \& Holstege, G. The neuronal control of micturition and its relation to the emotional motor system. Prog. Brain Res. 107, 113-126 (1996).

63 Blok, B., Sturms, L. \& Holstege, G. A PET study on cortical and subcortical control of pelvic floor musculature in women. J. Comp. Neurol. 389, 535-544 (1997).

64 Bohlen, J. G., Held, J. P., Sanderson, M. O. \& Ahlgren, A. The female orgasm: pelvic contractions. Arch. Sex. Behav. 11, 367-386 (1982). 
65 Shafik, A. The role of the levator ani muscle in evacuation, sexual performance and pelvic floor disorders. Int. Urogynecol. J. Pelvic Floor Dysfunct. 11, 361-376 (2000).

66 Boyer, S. C., Pukall, C. F. \& Chamberlain, S. M. Sexual arousal in women with provoked vestibulodynia: the application of laser Doppler imaging to sexual pain. J Sex Med 10, 1052-1064, doi:10.1111/j.1743-6109.2012.02855.x (2013).

67 Brauer, M., ter Kuile, M. M. \& Laan, E. Effects of appraisal of sexual stimuli on sexual arousal in women with and without superficial dyspareunia. Arch. Sex. Behav. 38, 476-485 (2009).

68 Cherner, R. A. \& Reissing, E. D. A psychophysiological investigation of sexual arousal in women with lifelong vaginismus. J Sex Med 10, 1291-1303 (2013).

69 Van Lankveld, J. J., Weijenborg, P. T. \& ter Kuile, M. M. Psychologic profiles of and sexual function in women with vulvar vestibulitis and their partners. Obstet. Gynecol. 88, 65-70 (1996).

70 Barlow, D. H. Causes of sexual dysfunction: the role of anxiety and cognitive interference. J. Consult. Clin. Psychol. 54, 140-148 (1986).

71 Payne, K. A., Binik, Y. M., Amsel, R. \& Khalife, S. When sex hurts, anxiety and fear orient attention towards pain. Eur J Pain 9, 427-436, doi:10.1016/j.ejpain.2004.10.003 (2005).

72 Lykins, A. D., Meana, M. \& Minimi, J. Visual attention to erotic images in women reporting pain with intercourse. J. Sex Res. 48, 43-52, doi:10.1080/00224490903556374 (2011).

73 Dewitte, M. Gender Differences in Liking and Wanting Sex: Examining the Role of Motivational Context and Implicit Versus Explicit Processing. Arch. Sex. Behav. 44, 1663-1674, doi:10.1007/s10508-014-0419-7 (2015).

74 Dewitte, M., Van Lankveld, J. \& Crombez, G. Understanding sexual pain: a cognitivemotivational account. Pain 152, 251-253, doi:10.1016/j.pain.2010.10.051 (2011).

75 Toates, F. An integrative theoretical framework for understanding sexual motivation, arousal, and behavior. J. Sex Res. 46, 168-193 (2009).

76 Gruenwald, I., Lowenstein, L., Gartman, I. \& Vardi, Y. Physiological changes in female genital sensation during sexual stimulation. J Sex Med 4, 390-394, doi:10.1111/j.1743-6109.2006.00415.x (2007).

77 Paterson, L. Q., Amsel, R. \& Binik, Y. M. Pleasure and pain: the effect of (almost) having an orgasm on genital and nongenital sensitivity. J Sex Med 10, 15311544, doi:10.1111/jsm.12144 (2013).

78 Bergeron, S. \& Lord, M. J. The integration of pelvi-perineal re-education and cognitive-behavioural therapy in the multidisciplinary treatment of the sexual pain disorders. The Journal of Sexual and Relationship Therapy 18, 135-141 (2003).

79 De Houwer, J., Thomas, S. \& Baeyens, F. Associative learning of likes and dislikes: a review of 25 years of research on human evaluative conditioning. Psychol. Bull. 127, 853-869 (2001).

80 Gregg, A. P., Seibt, B. \& Banaji, M. R. Easier done than undone: asymmetry in the malleability of implicit preferences. J. Pers. Soc. Psychol. 90, 1-20, doi:10.1037/0022-3514.90.1.1 (2006).

81 Huijding, J. \& de Jong, P. J. Implicit and Explicit Attitudes Toward Spiders: Sensitivity to Treatment and Predictive Value for Generalization of Treatment Effects. Cognitive Therapy and Research 33, 211-220 (2009). 
82 Strack, F. \& Deutsch, R. Reflective and impulsive determinants of social behavior. Personality and social psychology review: an official Journal of the Society for Personality and Social Psychology, Inc 8, 220-247, doi:10.1207/s15327957pspr0803_1 (2004).

83 Rinck, M. \& Becker, E. S. Spider fearful individuals attend to threat, then quickly avoid it: evidence from eye movements. J. Abnorm. Psychol. 115, 231-238, doi:10.1037/0021-843X.115.2.231 (2006).

84 Elmerstig, E., Wijma, B. \& Swahnberg, K. Prioritizing the partner's enjoyment: a population-based study on young Swedish women with experience of pain during vaginal intercourse. J. Psychosom. Obstet. Gynaecol. 34, 82-89, doi:10.3109/0167482X.2013.793665 (2013).

85 Elmerstig, E., Wijma, B. \& Swahnberg, K. Young Swedish women's experience of pain and discomfort during sexual intercourse. Acta Obstet Gynecol Scand 88, 98-103, doi:10.1080/00016340802620999 (2009).

86 Landry, T. \& Bergeron, S. How young does vulvo-vaginal pain begin? Prevalence and characteristics of dyspareunia in adolescents. J Sex Med 6, 927-935, doi:10.1111/j.1743-6109.2008.01166.x (2009).

de Jong, D. C. The role of attention in sexual arousal: implications for treatment of sexual dysfunction. J. Sex Res. 46, 237-248, doi:10.1080/00224490902747230 (2009).

88 de Ruiter, C. \& Brosschot, J. F. The emotional Stroop interference effect in anxiety: attentional bias or cognitive avoidance? Behav. Res. Ther. 32, 315-319 (1994).

89 MacLeod, C., Rutherford, E., Campbell, L., Ebsworthy, G. \& Holker, L. Selective attention and emotional vulnerability: assessing the causal basis of their association through the experimental manipulation of attentional bias. J. Abnorm. Psychol. 111, 107-123 (2002).

90 Both, S., Laan, E. \& Everaerd, W. Focusing "hot" or focusing "cool": attentional mechanisms in sexual arousal in men and women. J Sex Med 8, 167-179, doi:10.1111/j.1743-6109.2010.02051.x (2011).

91 Beard, C. \& Amir, N. Attention bias for sexual words in female sexual dysfunction. J. Sex Marital Ther. 36, 216-226, doi:10.1080/00926231003719616 (2010).

92 Baum, C., Schneider, R., Keogh, E. \& Lautenbacher, S. Different stages in attentional processing of facial expressions of pain: a dot-probe task modification. J Pain 14, 223-232, doi:10.1016/j.jpain.2012.11.001 (2013).

93 Melles, R. J. et al. Automatic and deliberate affective associations with sexual stimuli in women with lifelong vaginismus before and after therapist-aided exposure treatment. J Sex Med 11, 786-799, doi:10.1111/jsm.12360 (2014).

94 Smeets, E., Roefs, A., van Furth, E. \& Jansen, A. Attentional bias for body and food in eating disorders: increased distraction, speeded detection, or both? Behav. Res. Ther. 46, 229-238, doi:10.1016/j.brat.2007.12.003 (2008).

95 Greenwald, A. G., Nosek, B. A. \& Banaji, M. R. Understanding and using the implicit association test: I. An improved scoring algorithm. J. Pers. Soc. Psychol. 85, $197-$ 216 (2003).

96 Glashouwer, K. A., Smulders, F. T., de Jong, P. J., Roefs, A. \& Wiers, R. W. Measuring automatic associations: validation of algorithms for the Implicit Association Test (IAT) in a laboratory setting. J. Behav. Ther. Exp. Psychiatry 44, 105-113, doi:10.1016/j.jbtep.2012.07.015 (2013). 
97 Rosen, R. et al. The Female Sexual Function Index (FSFI): a multidimensional selfreport instrument for the assessment of female sexual function. J. Sex Marital Ther. 26, 191-208 (2000).

98 ter Kuile, M. M., Brauer, M. \& Laan, E. The Female Sexual Function Index (FSFI) and the Female Sexual Distress Scale (FSDS): psychometric properties within a Dutch population. J. Sex Marital Ther. 32, 289-304 (2006).

99 Jiann, B. P. Pitfalls in using the female sexual function index. J Sex Med 9, 1229, doi:10.1111/j.1743-6109.2011.02638.x (2012).

100 Tabachnick, B. G. \& Fidell, L. S. Using Multivariate Statistics (5th ed.). Upper Saddle River, NJ: Pearson Allyn \& Bacon. (2001).

101 Crombez, G., Van Ryckeghem, D. M., Eccleston, C. \& Van Damme, S. Attentional bias to pain-related information: a meta-analysis. Pain 154, 497-510, doi:10.1016/j.pain.2012.11.013 (2013).

102 Vasey, M. W., Harbaugh, C. N., Buffington, A. G., Jones, C. R. \& Fazio, R. H. Predicting return of fear following exposure therapy with an implicit measure of attitudes. Behav. Res. Ther. 50, 767-774, doi:10.1016/j.brat.2012.08.007 (2012).

103 Dewitte, M. \& Koster, E. H. Attentional breadth and proximity seeking in romantic attachment relationships. Br J Soc Psychol 53, 74-92, doi:10.1111/bjso.12013 (2014).

104 Notebaert, L., Clarke, P. J., Grafton, B. \& MacLeod, C. Validation of a novel attentional bias modification task: the future may be in the cards. Behav. Res. Ther. 65, 93-100, doi:10.1016/j.brat.2014.12.007 (2015).

105 Clerkin, E. M. \& Teachman, B. A. Training implicit social anxiety associations: an experimental intervention. J. Anxiety Disord. 24, 300-308, doi:10.1016/j.janxdis.2010.01.001 (2010).

106 Clerkin, E. M., Werntz, A. J., Magee, J. C., Lindgren, K. P. \& Teachman, B. A. Evaluating age differences in coping motives as a mediator of the link between social anxiety symptoms and alcohol problems. Psychology of addictive behaviors : journal of the Society of Psychologists in Addictive Behaviors 28, 880886, doi:10.1037/a0036813 (2014).

107 van Lankveld, J. J., Brewaeys, A. M., Ter Kuile, M. M. \& Weijenborg, P. T. Difficulties in the differential diagnosis of vaginismus, dyspareunia and mixed sexual pain disorder. J. Psychosom. Obstet. Gynaecol. 16, 201-209 (1995).

108 Binik, Y. M., Bergeron, S. \& Khalifé, S. Dyspareunia and Vaginismus: So-Called Sexual Pain. in: S. R. Leiblum (red.) Principles and Practice of Sex Therapy (2007).

109 ter Kuile, M. M. et al. Cognitive-behavioral therapy for women with lifelong vaginismus: process and prognostic factors. Behav. Res. Ther. 45, 359-373, doi:10.1016/j.brat.2006.03.013 (2007).

110 Leiblum, S. R. in (2000). Rosen, Raymond C (Ed), Leiblum, Sandra R (Ed), Principles and practice of sex therapy (3rd ed., pp.181 202). New York, NY, US: Guilford Press. xiv, $514 \mathrm{pp}$. (Guilford Press, 2000).

111 Beck, A. T. \& Clark, D. A. An information processing model of anxiety: automatic and strategic processes. Behav. Res. Ther. 35, 49-58 (1997).

112 Fazio, R. H. \& Towles-Schwen, T. in Dual-process theories in social psychology (ed \& Y. Trope S. Chaiken) 97-116 (Guilford, 1999). 
113 Huijding, J. \& de Jong, P. J. Specific predictive power of automatic spider-related affective associations for controllable and uncontrollable fear responses toward spiders. Behav. Res. Ther. 44, 161-176 (2006).

114 Van Bockstaele, B. et al. Differential predictive power of self report and implicit measures on behavioural and physiological fear responses to spiders. Int. J. Psychophysiol. 79, 166-174, doi:10.1016/j.ijpsycho.2010.10.003 (2011).

115 Teachman, B. A. \& Woody, S. R. Automatic processing in spider phobia: implicit fear associations over the course of treatment. J. Abnorm. Psychol. 112, 100-109 (2003).

116 Hermans, D., Vansteenwegen, D., Crombez, G., Baeyens, F. \& Eelen, P. Expectancylearning and evaluative learning in human classical conditioning: affective priming as an indirect and unobtrusive measure of conditioned stimulus valence. Behav. Res. Ther. 40, 217-234 (2002).

117 Karpinski, A. \& Steinman, R. B. The single category implicit association test as a measure of implicit social cognition. J. Pers. Soc. Psychol. 91, 16-32, doi:10.1037/0022-3514.91.1.16 (2006).

118 Huijding, J. \& de Jong, P. J. Automatic associations with the sensory aspects of smoking: positive in habitual smokers but negative in non-smokers. Addict. Behav. 31, 182-186 (2006).

119 Huijding, J., de Jong, P. J., Wiers, R. W. \& Verkooijen, K. Implicit and explicit attitudes toward smoking in a smoking and a nonsmoking setting. Addict. Behav. 30, 949-961, doi:10.1016/j.addbeh.2004.09.014 (2005).

120 Lang, P., Brandley, M. \& Cuthbert, B. International affective picture system (IAPS): Affective ratings of pictures and instruction manual. Technical Report A-8,. University of Florida, Gainesville, FL, (2008).

121 Laan, E., Everaerd, W., van Bellen, G. \& Hanewald, G. Women's sexual and emotional responses to male- and female-produced erotica. Arch. Sex. Behav. 23, 153-169 (1994).

122 Sheehan, D. V. et al. The Mini-International Neuropsychiatric Interview (M.I.N.I.): the development and validation of a structured diagnostic psychiatric interview for DSM-IV and ICD-10. J. Clin. Psychiatry 59 Suppl 20, 22-33;quiz 34-57 (1998).

123 van Vliet, I. M. \& de Beurs, E. [The MINI-International Neuropsychiatric Interview. A brief structured diagnostic psychiatric interview for DSM-IV en ICD-10 psychiatric disorders]. Tijdschrift voor Psychiatrie 49, 393-397 (2007).

124 Rosnow, R. L. \& Rosenthal, R. Computing contrasts, effect sizes, and counternulls on other people's published data: General procedures for research consumers. Pyschological Methods 1, 331-340 (1996).

125 Cohen, J. Statistical power analysis for the behavioral sciences. Revised edition. Academic press (1977).

126 Cohen, J. Statistical Power Analysis for the Behavioral Sciences (2nd ed.). New York: Academic Press. (1988).

127 de Jong, P. J., van Overveld, M. \& Borg, C. Giving in to arousal or staying stuck in disgust? Disgust-based mechanisms in sex and sexual dysfunction. J. Sex Res. 50, 247-262, doi:10.1080/00224499.2012.746280 (2013).

128 de Jong, P. J., van Overveld, M., Schultz, W. W., Peters, M. L. \& Buwalda, F. M. Disgust and contamination sensitivity in vaginismus and dyspareunia. Arch. Sex. Behav. 38, 244-252 (2009). 
129 De Houwer, J. \& Eelen, P. An affective variant of the Simon paradigm. Cognition and Emotion 12, 45-61 (1998).

130 Peterson, Z. D. \& Janssen, E. Ambivalent affect and sexual response: the impact of co-occurring positive and negative emotions on subjective and physiological sexual responses to erotic stimuli. Arch. Sex. Behav. 36, 793-807 (2007).

131 De Houwer, J. Contingency awareness and evaluative conditioning: when will it be enough? Conscious. Cogn. 10, 550-558; discussion 567-573 (2001).

132 Stevenson, R. J., Case, T. I. \& Oaten, M. J. Effect of self-reported sexual arousal on responses to sex-related and non-sex-related disgust cues. Arch. Sex. Behav. 40, 79-85, doi:10.1007/s10508-009-9529-z (2011).

133 Borg, C. \& de Jong, P. J. Feelings of disgust and disgust-induced avoidance weaken following induced sexual arousal in women. PloS one 7, e44111, doi:10.1371/journal.pone.0044111 (2012).

134 Laumann, E. O., Paik, A. \& Rosen, R. C. Sexual dysfunction in the United States: prevalence and predictors. JAMA 281, 537-544 (1999).

135 Christensen, B. S. et al. Sexual dysfunctions and difficulties in denmark: prevalence and associated sociodemographic factors. Arch. Sex. Behav. 40, 121-132, doi:10.1007/s10508-010-9599-y (2011).

136 A.R., F.-M. \& K.S., F.-M. Sexual disabilities, problems and satisfaction in 18-74 year old Swedes. Scand J Sex 2, 79-105 (1999).

137 Kadri, N., McHichi Alami, K. H. \& McHakra Tahiri, S. Sexual dysfunction in women: population based epidemiological study. Archives of women's mental health 5, 59-63, doi:10.1007/s00737-002-0141-7 (2002).

138 Oberg, K. \& Sjogren Fugl-Meyer, K. On Swedish women's distressing sexual dysfunctions: some concomitant conditions and life satisfaction. J Sex Med 2, 169-180, doi:10.1111/j.1743-6109.2005.20226.x (2005).

139 Dogan, S. Vaginismus and accompanying sexual dysfunctions in a Turkish clinical sample. J Sex Med 6, 184-192, doi:10.1111/j.1743-6109.2008.01048.x (2009).

140 Oniz, A., Keskinoglu, P. \& Bezircioglu, I. The prevalence and causes of sexual problems among premenopausal turkish women J Sex Med 4, 1575-1581 (2007).

141 Ozdemir, O., Simsek, F., Ozkardes, S., Incesu, C. \& Karakoc, B. The unconsummated marriage: its frequency and clinical characteristics in a sexual dysfunction clinic. J. Sex Marital Ther. 34, 268-279, doi:10.1080/00926230701866380 (2008).

142 Yasan, A. \& Gurgen, F. Marital satisfaction, sexual problems, and the possible ifficulties on sex therapy in traditional Islamic culture. J. Sex Marital Ther. 35, 68-75, doi:10.1080/00926230802525687 (2009).

143 Catalan, J., Hawton, K. \& Day, A. Couples referred to a sexual dysfunction clinic. Psychological and physical morbidity. Br. J. Psychiatry 156, 61-67 (1990).

144 Nobre, P. J., Pinto-Gouveia, J. \& Gomes, F. A. Prevalence and comorbidity of sexual dysfunctions in a Portuguese clinical sample J. Sex Marital Ther. 32, 173-182 (2006).

145 Reissing, E. D. Vaginismus: Evaluation and Management, 229. In: A. T. Goldstein, C. F. Pukall, I. Goldstein (red.). Sexual Medicine, Female Sexual Pain Disorders (2009).

146 Reissing, E. D. Consultation and treatment history and causal attributions in an online sample of women with lifelong and acquired vaginismus. J Sex Med 9, 251-258, doi:10.1111/j.1743-6109.2011.02534.x (2012). 
147 ter Kuile, M. M. \& Weijenborg, P. T. A cognitive-behavioral group program for women with vulvar vestibulitis syndrome (VVS): factors associated with treatment success. J. Sex Marital Ther. 32, 199-213, doi:10.1080/00926230600575306 (2006).

148 Rust, J. \& Golombok, S. The GRISS: a psychometric instrument for the assessment of sexual dysfunction. Arch. Sex. Behav. 15, 157-165 (1986).

149 van Lankveld, J. J. D. M. \& ter Kuile, M. M. The Golombok Rust Inventory of Sexual Satisfaction (GRISS): Predictive validity and construct validity in a Dutch population Pers Individ Differ 26, 1005-1023 (1999).

150 ter Kuile, M. M., van Lankveld, J. J., Kalkhoven, P. \& van Egmond, M. The Golombok Rust Inventory of Sexual Satisfaction (GRISS): psychometric properties within a Dutch population. J. Sex Marital Ther. 25, 59-71 (1999).

151 van Overveld, M. et al. The Sexual Disgust Questionnaire; a Psychometric Study and a First Exploration in Patients with Sexual Dysfunctions. J Sex Med 10, 396407, doi:10.1111/j.1743-6109.2012.02979.x (2013).

152 Baron, R. M. \& Kenny, D. A. The moderator-mediator variable distinction in social psychological research: conceptual, strategic, and statistical considerations. J. Pers. Soc. Psychol. 51, 1173-1182 (1986).

153 Van Buuren, S., Brand, J. P. L., Groothuis-Oudshoorn, C. G. M. \& Rubin, D. B. Fully conditional specification in multivariate imputation. J Stat Comput Simul 76, 1049-1064 (2006).

154 Enders, C. K. Applied missing data analysis. (Guilford, 2010).

155 Preacher, K. J. \& Hayes, A. F. Asymptotic and resampling strategies for assessing and comparing indirect effects in multiple mediator models. Behavior Research Methods 40, 879-891 (2008).

156 Kazdin, A. E. Mediators and mechanisms of change in psychotherapy research. Annual review of clinical psychology 3, 1-27, doi:10.1146/annurev.clinpsy.3.022806.091432 (2007).

157 Brauer, M., ter Kuile, M. M., Laan, E. \& Trimbos, B. Cognitive-affective correlates and predictors of superficial dyspareunia. J. Sex Marital Ther. 35, 1-24 (2009).

158 Puppo, V. Anatomy and physiology of the clitoris, vestibular bulbs, and labia minora with a review of the female orgasm and the prevention of female sexual dysfunction. Clin. Anat. 26, 134-152, doi:10.1002/ca.22177 (2013).

159 Levin, R. The physiology of sexual arousal in the human female: a recreational and procreational synthesis. Arch. Sex. Behav. 31, 405-411 (2002).

160 Farmer, M. A. \& Meston, C. M. Predictors of genital pain in young women. Arch. Sex. Behav. 36, 831-843 (2007).

161 Both, S. \& Laan, E. Simultaneous measurement of pelvic floor muscle activity and vaginal blood flow: a pilot study. J Sex Med 4, 690-701, doi:10.1111/j.17436109.2007.00457.x (2007).

162 Vardi, Y., Gruenwald, I., Sprecher, E., Gertman, I. \& Yartnitsky, D. Normative values for female genital sensation. Urology 56, 1035-1040 (2000).

163 Pukall, C. F., Binik, Y. M. \& Khalife, S. A new instrument for pain assessment in vulvar vestibulitis syndrome. J. Sex Marital Ther. 30, 69-78, doi:10.1080/00926230490275065 (2004).

164 Bohm-Starke, N., Hilliges, M., Brodda-Jansen, G., Rylander, E. \& Torebjork, E. Psychophysical evidence of nociceptor sensitization in vulvar vestibulitis syndrome. Pain 94, 177-183 (2001). 
165 Schaefer, A., Frédéric, N., Sanchez, X. \& Philippot, P. Assessing the effectiveness of a large database of emotion-eliciting films: A new tool for emotion researchers. Cognition and emotion 2010 (2010).

166 Basson, R. A model of women's sexual arousal. J. Sex Marital Ther. 28, 1-10, doi:10.1080/009262302317250963 (2002).

167 Heiman, J. R. Female sexual response patterns. Interactions of physiological, affective, and contextual cues. Arch. Gen. Psychiatry 37, 1311-1316 (1980).

168 van Lankveld, J. et al. The Partner's Presence in the Sex Research Lab Differentially Affects Sexual Arousal in Women and Men. J Sex Med 11, 697-708, doi:10.1111/jsm.12406 (2014).

169 Farmer, M. A. et al. Psychophysical properties of female genital sensation. Pain 154, 2277-2286, doi:10.1016/j.pain.2013.05.028 (2013).

170 Landry, T. \& Bergeron, S. Biopsychosocial factors associated with dyspareunia in a community sample of adolescent girls. Arch. Sex. Behav. 40, 877-889, doi:10.1007/s10508-010-9637-9 (2011).

171 Khandker, M. et al. The influence of depression and anxiety on risk of adult onset vulvodynia. J Womens Health (Larchmt) 20, 1445-1451, doi:10.1089/jwh.2010.2661 (2011).

172 Thomten, J., Lundahl, R., Stigenberg, K. \& Linton, S. Fear avoidance and pain catastrophizing among women with sexual pain. Womens Health Data Book 10, 571-581, doi:10.2217/whe.14.51 (2014).

173 Bergeron, S., Likes, W. M. \& Steben, M. Psychosexual aspects of vulvovaginal pain. Best practice \& research. Clinical Obstetrics \& Gynaecology 28, 991-999, doi:10.1016/j.bpobgyn.2014.07.007 (2014).

174 Meana, M. \& Lykins, A. Negative affect and somatically focused anxiety in young women reporting pain with intercourse. J. Sex Res. 46, 80-88, doi:10.1080/00224490802624422 (2009).

175 Reissing, E. D., Binik, Y. M., Khalife, S., Cohen, D. \& Amsel, R. Etiological correlates of vaginismus: sexual and physical abuse, sexual knowledge, sexual self-schema, and relationship adjustment. J. Sex Marital Ther. 29, 47-59, doi:10.1080/713847095 (2003).

176 Peerdeman, K. J. et al. Relieving patients' pain with expectation interventions: a meta-analysis. Pain 157, 1179-1191, doi:10.1097/j.pain.0000000000000540 (2016).

177 Peerdeman, K. J., van Laarhoven, A. I., Peters, M. L. \& Evers, A. W. An Integrative Review of the Influence of Expectancies on Pain. Frontiers in psychology 7, 1270, doi:10.3389/fpsyg.2016.01270 (2016).

178 van Laarhoven, A. I. et al. Induction of nocebo and placebo effects on itch and pain by verbal suggestions. Pain 152, 1486-1494, doi:10.1016/j.pain.2011.01.043 (2011).

179 Aouad, M. T. et al. Predictors of postoperative pain and analgesic requirements following abdominal hysterectomy: an observational study. Journal of Anesthesia 30, 72-79, doi:10.1007/s00540-015-2090-0 (2016).

180 Cormier, S., Lavigne, G. L., Choiniere, M. \& Rainville, P. Expectations predict chronic pain treatment outcomes. Pain 157, 329-338, doi:10.1097/j.pain.0000000000000379 (2016).

181 Brignardello-Petersen, R. Previous bad experience, propensity to anxiety, and pain expectations may be associated with fear and anxiety when undergoing tooth extractions. J. Am. Dent. Assoc. 148, e4, doi:10.1016/j.adaj.2016.11.015 (2017). 
182 Arntz, A., van Eck, M. \& Heijmans, M. Predictions of dental pain: the fear of any expected evil, is worse than the evil itself. Behav. Res. Ther. 28, 29-41 (1990).

183 George, J. M., Scott, D. S., Turner, S. P. \& Gregg, J. M. The effects of psychological factors and physical trauma on recovery from oral surgery. J. Behav. Med. 3, 291-310 (1980).

184 Boston, A. \& Sharpe, L. The role of threat-expectancy in acute pain: effects on attentional bias, coping strategy effectiveness and response to pain. Pain 119, 168-175, doi:10.1016/j.pain.2005.09.032 (2005).

185 Klages, U., Ulusoy, O., Kianifard, S. \& Wehrbein, H. Dental trait anxiety and pain sensitivity as predictors of expected and experienced pain in stressful dental procedures. Eur. J. Oral Sci. 112, 477-483, doi:10.1111/j.16000722.2004.00167.x (2004).

186 Rhudy, J. L., Williams, A. E., McCabe, K. M., Russell, J. L. \& Maynard, L. J. Emotional control of nociceptive reactions (ECON): do affective valence and arousal play a role? Pain 136, 250-261, doi:10.1016/j.pain.2007.06.031 (2008).

187 Bianchi-Demicheli, F. \& Ortigue, S. Toward an understanding of the cerebral substrates of woman's orgasm. Neuropsychologia 45, 2645-2659, doi:10.1016/j.neuropsychologia.2007.04.016 (2007).

188 Melles, R. J., Dewitte, M. D., Ter Kuile, M. M., Bonnemayer, C. \& Peters, M. M. L. The Vaginal Pressure Inducer: A New Device to Test the (Un)pleasurableness and Tolerance of Vaginal Pressure and the Influence of Sexual Stimuli. J. Sex Marital Ther. 44, 189-200, doi:10.1080/0092623X.2017.1342728 (2018).

189 Dewitte, M., Schepers, J. \& Melles, R. The Effects of Partner Presence and Sexual Stimulation on the Appraisal of Vaginal Pressure and Sexual Arousal. J Sex Med 15, 539-549, doi:10.1016/j.jsxm.2018.02.009 (2018).

190 Spielberger, C. D. State-Trait Anxiety Inventory: Bibliography 2nd edn, (C.A.: Consulting Psychologists Press, 1989).

191 van der Ploeg, H. M., Defares, P. B. \& Spielberger, C. D. Handleiding bij de ZelfBeoordelings Vragenlijst ZBV. Een Nederlandse bewerking van de Spielberger State-Trait Anxiety Inventory STAI-DY. Pearson Assessment and Information B.V., Amsterdam, (2000).

192 McCracken, L. M. 'Attention' to pain in persons with chronic pain: a behavioral approach. Behavior Therapy 28, 271-284 (1997).

193 Roelofs, J., Peters, M. L., Muris, P. \& Vlaeyen, J. W. Dutch version of the Pain Vigilance and Awareness Questionnaire: validity and reliability in a pain-free population. Behav. Res. Ther. 40, 1081-1090 (2002).

194 MacKinnon, D. P., Lockwood, C. M., Hoffman, J. M., West, S. G. \& Sheets, V. A comparison of methods to test mediation and other intervening variable effects. Psychological methods 7, 83-104 (2002).

195 Craske, M. G., Treanor, M., Conway, C. C., Zbozinek, T. \& Vervliet, B. Maximizing exposure therapy: an inhibitory learning approach. Behav. Res. Ther. 58, 10-23, doi:10.1016/j.brat.2014.04.006 (2014).

196 Ter Kuile, M. M., Melles, R. J., Tuijnman-Raasveld, C. C., de Groot, H. E. \& van Lankveld, J. J. Therapist-Aided Exposure for Women with Lifelong Vaginismus: Mediators of Treatment Outcome: A Randomized Waiting List Control Trial. J Sex Med 12, 1807-1819, doi:10.1111/jsm.12935 (2015).

197 Whipple, B. \& Komisaruk, B. R. Elevation of pain threshold by vaginal stimulation in women. Pain 21, 357-367 (1985). 
198 Whipple, B. \& Komisaruk, B. R. Analgesia produced in women by genital selfstimulation. J. Sex Res. 24, 130-140, doi:10.1080/00224498809551403 (1988).

199 Gawronski, B., LeBel, E. P. \& Peters, K. R. What Do Implicit Measures Tell Us?: Scrutinizing the Validity of Three Common Assumptions. Perspectives on psychological science : a journal of the Association for Psychological Science 2, 181-193, doi:10.1111/j.1745-6916.2007.00036.x (2007).

200 Dunkley, C. R. \& Brotto, L. A. Psychological Treatments for Provoked Vestibulodynia: Integration of Mindfulness-Based and Cognitive Behavioral Therapies. J. Clin. Psychol. 72, 637-650, doi:10.1002/jclp.22286 (2016).

201 Nosek, B. A. et al. National differences in gender-science stereotypes predict national sex differences in science and math achievement. . Proc. Natl. Acad. Sci. U. S. A. 106, 10593-10597, doi:10.1073/pnas.0809921106 (2009).

202 Sanchez, D. T., Kiefer, A. K. \& Ybarra, O. Sexual submissiveness in women: costs for sexual autonomy and arousal. Personality \& Social Psychology Bulletin 32, 512524, doi:10.1177/0146167205282154 (2006).

203 Sanchez, D. T., Crocker, J. \& Boike, K. R. Doing gender in the bedroom: investing in gender norms and the sexual experience. Personality \& Social Psychology Bulletin 31, 1445-1455, doi:10.1177/0146167205277333 (2005).

204 Dewitte, M., De Schryver, M., Heider, N. \& De Houwer, J. The Actual and Ideal Sexual Self Concept in the Context of Genital Pain Using Implicit and Explicit Measures. J Sex Med 14, 702-714, doi:10.1016/j.jsxm.2017.03.246 (2017).

205 Elmerstig, E., Wijma, B. \& Bertero, C. Why do young women continue to have sexual intercourse despite pain? J. Adolesc. Health 43, 357-363 (2008).

206 Bergeron, S., Khalife, S., Dupuis, M. J. \& McDuff, P. A randomized clinical trial comparing group cognitive-behavioral therapy and a topical steroid for women with dyspareunia. J. Consult. Clin. Psychol. 84, 259-268, doi:10.1037/ccp0000072 (2016).

207 Backman, H., Widenbrant, M., Bohm-Starke, N. \& Dahlof, L. G. Combined physical and psychosexual therapy for provoked vestibulodynia-an evaluation of a multidisciplinary treatment model. J. Sex Res. 45, 378-385, doi:10.1080/00224490802398365 (2008).

208 Masheb, R. M., Kerns, R. D., Lozano, C., Minkin, M. J. \& Richman, S. A randomized clinical trial for women with vulvodynia: Cognitive-behavioral therapy vs. supportive psychotherapy. Pain 141, 31-40 (2009).

209 Bergeron, S., Khalife, S., Glazer, H. I. \& Binik, Y. M. Surgical and behavioral treatments for vestibulodynia: two-and-one-half year follow-up and predictors of outcome. Obstet. Gynecol. 111, 159-166, doi:10.1097/01.AOG.0000295864.76032.a7 (2008).

210 Meulders, A., Karsdorp, P. A., Claes, N. \& Vlaeyen, J. W. S. Comparing Counterconditioning and Extinction as Methods to Reduce Fear of MovementRelated Pain. J Pain 16, 1353-1365, doi:10.1016/j.jpain.2015.09.007 (2015).

211 Gast, A. \& De Houwer, J. The influence of extinction and counterconditioning instructions on evaluative conditioning effects. Learn. Motiv. 44, 312-325 (2013).

212 Engelhard, I. M., Leer, A., Lange, E. \& Olatunji, B. O. Shaking that icky feeling: effects of extinction and counterconditioning on disgust-related evaluative learning. Behav Ther 45, 708-719, doi:10.1016/j.beth.2014.04.003 (2014). 
213 Kerkhof, I., Vansteenwegen, D., Baeyens, F. \& Hermans, D. Counterconditioning: an effective technique for changing conditioned preferences. Experimental Psychology 58, 31-38, doi:10.1027/1618-3169/a000063 (2011).

214 Raes, A. K. \& De Raedt, R. The effect of counterconditioning on evaluative responses and harm expectancy in a fear conditioning paradigm. Behavior Therapy 43, 757-767, doi:10.1016/j.beth.2012.03.012 (2012).

215 Claes, N., Crombez, G. \& Vlaeyen, J. W. Pain-avoidance versus reward-seeking: an experimental investigation. Pain 156, 1449-1457, doi:10.1097/j.pain.0000000000000116 (2015).

216 Claes, N., Karos, K., Meulders, A., Crombez, G. \& Vlaeyen, J. W. S. Competing goals attenuate avoidance behavior in the context of pain. J Pain 15, 1120-1129, doi:10.1016/j.jpain.2014.08.003 (2014).

217 Bartels, D. J. P. et al. Minimizing nocebo effects by conditioning with verbal suggestion: A randomized clinical trial in healthy humans. PloS one 12, e0182959, doi:10.1371/journal.pone.0182959 (2017).

218 Linton, S. J. \& Fruzzetti, A. E. A hybrid emotion-focused exposure treatment for chronic pain: A feasibility study. Scandinavian Journal of Pain 5, 151-158, doi:10.1016/j.sjpain.2014.05.008 (2014).

219 Martinez-Calderon, J. et al. The role of psychological factors in the perpetuation of pain intensity and disability in people with chronic shoulder pain: a systematic review. BMJ open 8, e020703, doi:10.1136/bmjopen-2017-020703 (2018).

220 Goubert, L. \& Trompetter, H. Towards a science and practice of resilience in the face of pain. Eur J Pain 21, 1301-1315, doi:10.1002/ejp.1062 (2017).

221 Fava, G. A., Cosci, F., Guidi, J. \& Tomba, E. Well-being therapy in depression: New insights into the role of psychological well-being in the clinical process. Depress. Anxiety 34, 801-808, doi:10.1002/da.22629 (2017).

222 Steptoe, A., Dockray, S. \& Wardle, J. Positive affect and psychobiological processes relevant to health. J. Pers. 77, 1747-1776, doi:10.1111/j.14676494.2009.00599.x (2009).

223 Hanssen, M. M., Peters, M. L., Boselie, J. J. \& Meulders, A. Can positive affect attenuate (persistent) pain? State of the art and clinical implications. Current rheumatology reports 19, 80, doi:10.1007/s11926-017-0703-3 (2017).

224 Hanssen, M. M., Peters, M. L., Vlaeyen, J. W., Meevissen, Y. M. \& Vancleef, L. M. Optimism lowers pain: evidence of the causal status and underlying mechanisms. Pain 154, 53-58, doi:10.1016/j.pain.2012.08.006 (2013).

225 Hanssen, M. M., Vancleef, L. M., Vlaeyen, J. W. \& Peters, M. L. More optimism, less pain! The influence of generalized and pain-specific expectations on experienced cold-pressor pain. J. Behav. Med. 37, 47-58, doi:10.1007/s10865012-9463-8 (2014).

226 Basson, R. The recurrent pain and sexual sequelae of provoked vestibulodynia: a perpetuating cycle. J Sex Med 9, 2077-2092, doi:10.1111/j.17436109.2012.02803.x (2012).

227 Guillet, A. D., Cirino, N. H., Hart, K. D. \& Leclair, C. M. Mindfulness-Based Group Cognitive Behavior Therapy for Provoked Localized Vulvodynia: A Randomized Controlled Trial. Journal of lower Genital Tract Disease, doi:10.1097/LGT.0000000000000456 (2019).

228 Rosenbaum, T. Y. An integrated mindfulness-based approach to the treatment of women with sexual pain and anxiety: promoting autonomy and mind/body 
connection. Sexual and Relationship Therapy, 28:1-2 28, 20-28, doi:DOI: 10.1080/14681994.2013.764981 (2013).

229 Impett, E. A. \& Peplau, L. A. Sexual compliance: gender, motivational, and relationship perspectives. J. Sex Res. 40, 87-100, doi:10.1080/00224490309552169 (2003).

230 Hite, S., The Hite Report: A National Study of Female Sexuality, New York, Dell, (1976).

231 Lloyd, E. A., The Case of the Female Orgasm: Bias in the Science of Evolution, Cambridge, Harvard University Press, (2005).

232 O'Connell, H. E., Sanjeevan, K. V. \& Hutson, J. M. Anatomy of the clitoris. J. Urol. 174, 1189-1195 (2005).

233 van Lunsen, R. \& Laan, E. Seks! Een leven lang leren., Amsterdam, Promoteus, (2017).

234 Ayling, K. \& Ussher, J. M. "If sex hurts, am I still a woman?" the subjective experience of vulvodynia in hetero-sexual women. Arch. Sex. Behav. 37, 294304, doi:10.1007/s10508-007-9204-1 (2008).

235 Impett, E. A., Gable, S. L. \& Peplau, L. A. Giving up and giving in: the costs and benefits of daily sacrifice in intimate relationships. J. Pers. Soc. Psychol. 89, 327 344, doi:10.1037/0022-3514.89.3.327 (2005).

236 Impett, E. A. et al. Moving toward more perfect unions: daily and long-term consequences of approach and avoidance goals in romantic relationships. $J$. Pers. Soc. Psychol. 99, 948-963, doi:10.1037/a0020271 (2010).

237 Impett, E. A., Strachman, A., Finkel, E. J. \& Gable, S. L. Maintaining sexual desire in intimate relationships: the importance of approach goals. J. Pers. Soc. Psychol. 94, 808-823, doi:10.1037/0022-3514.94.5.808 (2008).

238 Muise, A., Impett, E. A. \& Desmarais, S. Getting it on versus getting it over with: sexual motivation, desire, and satisfaction in intimate bonds. Personality \& social psychology bulletin 39, 1320-1332, doi:10.1177/0146167213490963 (2013).

239 Rosen, N. O. et al. Sexual Cues Mediate the Daily Associations Between Interpersonal Goals, Pain, and Well-being in Couples Coping With Vulvodynia. nn. Behav. Med. 52, 216-227, doi:10.1093/abm/kax046 (2018).

240 Rosen, N. O., Muise, A., Bergeron, S., Impett, E. A. \& Boudreau, G. K. Approach and Avoidance Sexual Goals in Couples with Provoked Vestibulodynia: Associations with Sexual, Relational, and Psychological Well-Being. J Sex Med 12, 1781-1790, doi:10.1111/jsm.12948 (2015).

241 Claes, N., Crombez, G., Meulders, A. \& Vlaeyen, J. W. Between the Devil and the Deep Blue Sea: Avoidance-Avoidance Competition Increases Pain-Related Fear and Slows Decision-Making. J Pain 17, 424-435, doi:10.1016/j.jpain.2015.12.005 (2016).

242 Impett, E. A., Gable, S. L. \& Peplau, L. A. Approach and avoidance sexual motives: Implications for personal and interpersonal well-being. Personal Relationships 12, 465-482 (2005).

243 Murphy, R., Straebler, S., Cooper, Z. \& Fairburn, C. G. Cognitive behavioral therapy for eating disorders. Psychiatr. Clin. North Am. 33, 611-627, doi:10.1016/j.psc.2010.04.004 (2010).

244 Masters, W. H. \& Johnson, V. E. Human sexual response. (Little Brown, 1966).

245 Strassberg, D. S. \& Lowe, K. Volunteer bias in sexuality research. Arch. Sex. Behav. 24, 369-382 (1995). 
246 Wolchik, S. A., Braver, S. L. \& Jensen, K. Volunteer bias in erotica research: effects of intrusiveness of measure and sexual background. Arch. Sex. Behav. 14, 93-107 (1985).

247 Wolchik, S. A., Spencer, S. L. \& Lisi, I. S. Volunteer bias in research employing vaginal measures of sexual arousal. Arch. Sex. Behav. 12, 399-408 (1983).

248 De Houwer, J. \& De Bruycker, E. The implicit association test outperforms the extrinsic affective Simon task as an implicit measure of inter-individual differences in attitudes. $B r \quad J$ Soc Psychol 46, 401-421, doi:10.1348/014466606X130346 (2007).

249 Armstrong, T. \& Olatunji, B. O. Eye tracking of attention in the affective disorders: a meta-analytic review and synthesis. Clin. Psychol. Rev. 32, 704-723, doi:10.1016/j.cpr.2012.09.004 (2012).

250 Brauer, M. et al. Attentional and affective processing of sexual stimuli in women with hypoactive sexual desire disorder. Arch. Sex. Behav. 41, 891-905, doi:10.1007/s10508-011-9820-7 (2012).

251 Dewitte, M. Partner congruence in genital pain. Arch. Sex. Behav. (accepted).

252 Puppo, V. Embryology and anatomy of the vulva: the female orgasm and women's sexual health. Eur. J. Obstet. Gynecol. Reprod. Biol. 154, 3-8, doi:10.1016/j.ejogrb.2010.08.009 (2011).

253 Reissing, E. D., Armstrong, H. L. \& Allen, C. Pelvic floor physical therapy for lifelong vaginismus: a retrospective chart review and interview study. J. Sex Marital Ther. 39, 306-320, doi:10.1080/0092623X.2012.697535 (2013).

254 Feiring, C., Simon, V. A. \& Cleland, C. M. Childhood sexual abuse, stigmatization, internalizing symptoms, and the development of sexual difficulties and dating aggression. J. Consult. Clin. Psychol. 77, 127-137, doi:10.1037/a0013475 (2009).

255 Nicolai, N. r. Handboek psychotherapie na seksueel misbruik. (de Tijdstroom, 2003).

256 Davis, S. N. et al. A prospective 2-year examination of cognitive and behavioral correlates of provoked vestibulodynia outcomes. Clin. J. Pain 31, 333-341, doi:10.1097/AJP.0000000000000128 (2015).

257 Desrochers, G., Bergeron, S., Khalife, S., Dupuis, M. J. \& Jodoin, M. Provoked vestibulodynia: psychological predictors of topical and cognitive-behavioral treatment outcome. Behav. Res. Ther. 48, 106-115, doi:10.1016/j.brat.2009.09.014 (2010).

258 Seal, B. N. \& Meston, C. M. The Impact of Body Awareness on Women's Sexual Health: A Comprehensive Review. Sexual medicine reviews, doi:10.1016/j.sxmr.2018.03.003 (2018).

259 Schrooten, M. G., Vlaeyen, J. W. \& Morley, S. Psychological interventions for chronic pain: reviewed within the context of goal pursuit. Pain Manage 2, 141-150 (2012).

260 Domenici, L. et al. Vulvodynia: current opinion and treatment strategies. Minerva Ginecol. 68, 727-732 (2016).

261 van Lankveld, J. J. D. M., Ter Kuile, M. M., Kenter, G. G., van Hall, E. V. \& Weijenborg, P. T. M. Seksuele klachten en ervaringen met seksueel en fysiek geweld bij gynaecologische patiënten. Ned. Tijdschr. Geneeskd. 140, 1903-1906 (1996).

262 Moller, L., Josefsson, A., Bladh, M., Lilliecreutz, C. \& Sydsjo, G. Reproduction and mode of delivery in women with vaginismus or localised provoked vestibulodynia: a Swedish register-based study. BJOG : an international journal 
of obstetrics and gynaecology 122, 329-334, doi:10.1111/1471-0528.12946 (2015).

263 Leusink, P., Teunissen, D., Lucassen, P. L., Laan, E. T. \& Lagro-Janssen, A. L. Facilitators and barriers in the diagnostic process of vulvovaginal complaints (vulvodynia) in general practice: a qualitative study. Eur J Gen Pract 24, 92-98, doi:10.1080/13814788.2017.1420774 (2018).

264 Graziottin, A., Gambini, D. \& Bertolasi, L. Genital and sexual pain in women. Handbook of Clinical Neurology 130, 395-412, doi:10.1016/B978-0-44463247-0.00023-7 (2015).

265 Melles, R. J. in European Association for Behavioural and Cognitive Therapies, Annual Congress, Reykjavik, Iceland.

266 Brauer, M., van Lunsen, R. H., Laan, E. T. \& Burger, M. P. A Qualitative Study on Experiences After Vulvar Surgery in Women With Lichen Sclerosus and Sexual Pain. J Sex Med 13, 1080-1090, doi:10.1016/j.jsxm.2016.04.072 (2016).

267 Brauer, M., van Lunsen, R., Burger, M. \& Laan, E. Motives for Vulvar Surgery of Women with Lichen Sclerosus. J Sex Med 12, 2462-2473, doi:10.1111/jsm.13052 (2015).

268 Schroder, M. et al. Clitoral therapy device for treatment of sexual dysfunction in irradiated cervical cancer patients. Int. J. Radiat. Oncol. Biol. Phys. 61, 10781086, doi:10.1016/j.ijrobp.2004.07.728 (2005).

269 van Rees, B., Spiering, M. \& Laan, E. Orgasmeconsistentie van lesbische en heteroseksuele vrouwen tijdens partnerseks: De rol van clitorale stimulatie. Tijdschrift voor Seksuologie 40, 68-75 (2016). 


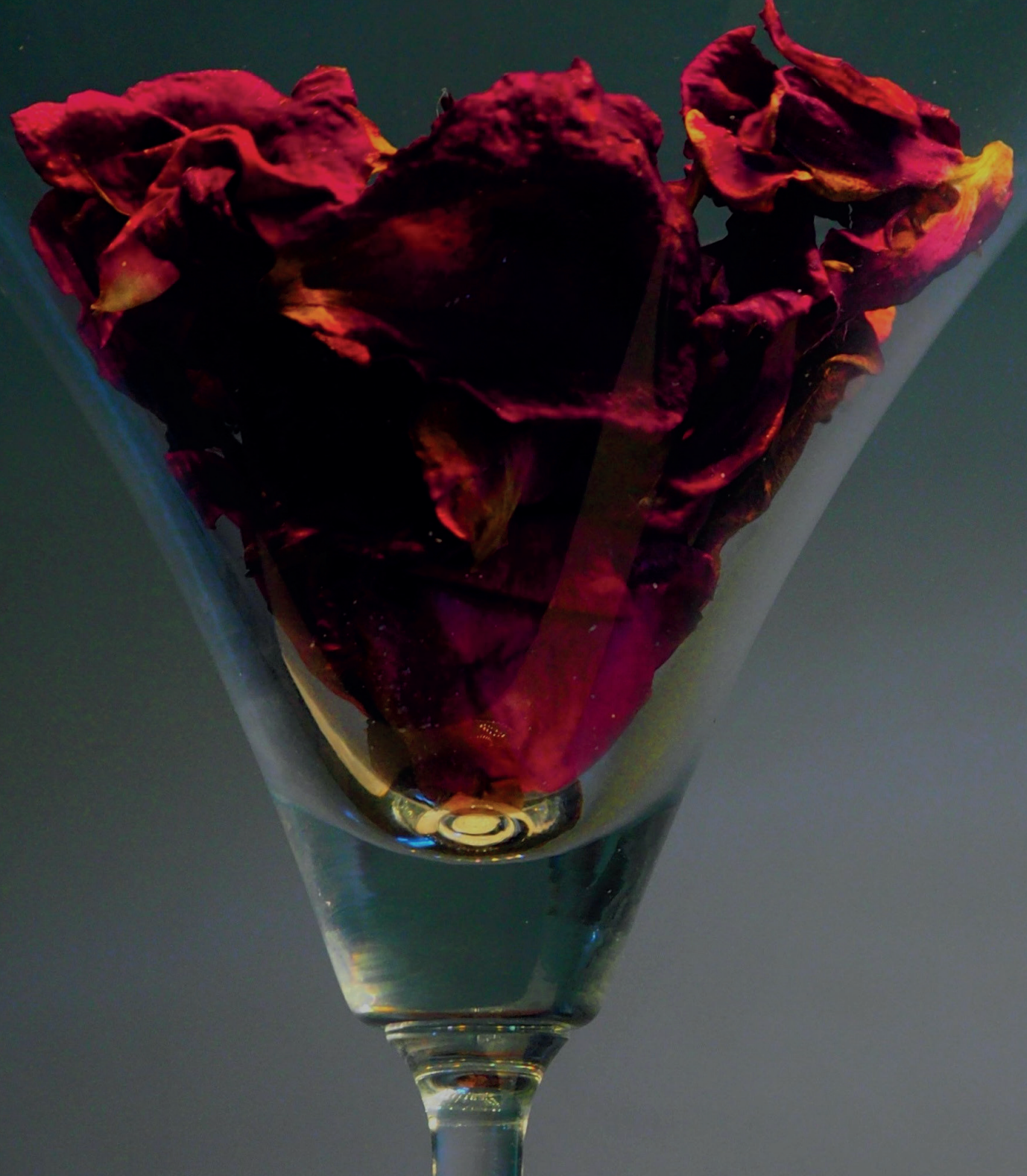

Dankwoord 
Eindelijk! Het boekje is af! Echt waar! Hierbij wil ik graag iedereen bedanken die mij heeft bijgestaan in de totstandkoming van dit proefschrift.

Allereerst gaat mijn dank uit naar mijn patienten. Zonder jullie was ik nooit aan dit proefschrift begonnen. Jullie hebben me geholpen de puzzelstukjes te ontrafelen..... De onderzoeksvragen van dit proefschrift zijn daar direct uit voortgekomen. Zonder jullie vertrouwen en openhartigheid was dit niet mogelijk geweest.

Voorts gaat mijn dank uit naar mijn stagiares. Maartje, Charlotte, Alicia, Eva, Anouska, Karina, Rianne, Stina, Jeanine, Emmy, Lisa, Laura V., Thera, Laura van E, Mikal, Rosie, Jetske. Veel van jullie hebben een geweldige bijdrage geleverd aan het het uitvoeren van de studies, het werven en selecteren van proefpersonen, het verzamelen van de data, etc. etc. Speciale dank gaat uit naar Lisette, de eerste stagiare die met de VPI heeft gewerkt. Lisette, ik ben je enorm dankbaar voor je grote betrokkenheid en inzet. Door jouw zorgvuldigheid hebben we met deze pilotversie van de VPI, ondanks alle mankementen, een zeer bruikbare en uitgebreide dataset verzameld.

Graag wil ik mijn collega's van de instrumentele dienst bedanken voor hun constructieve bijdragen en expertise. Speciale dank gaat uit naar Charlie en Jacco, die altijd bereid waren om tijd vrij te maken en mee te denken, veel dank voor jullie inspanning om echt te begrijpen wat ik graag wilde en zo een brug te slaan tussen praktijk en techniek. Charlie, eigenlijk hadden we onze creatieve ontwerpen met w.c.- rolletjes, pvc-buisjes,

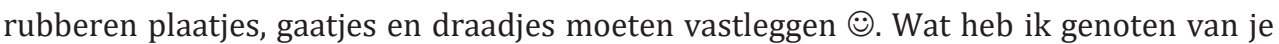
tomeloze inzet en creativiteit....en je moed om als man in dit onderwerp te duiken!

Speciale dank gaat ook uit naar mijn collega-seksuoloog, Thea, die heeft geholpen met het ontwerpen van het "broekje", een essentieel onderdeel van de VPI. Thea, jij hebt een unieke bijdrage geleverd aan het tot stand komen van de VPI door je inhoudelijke deskundigheid, nuchtere oplossingsgerichtheid en je bijzondere naaikunst. Wat hebben we gelachen toen ik in jouw bed terechtkwam om ons eerste ontwerp van het broekje te passen!

De dames van het Pelvic Care Team Maastricht, Marion en Patricia, wil ik graag hartelijk bedanken voor hun bereidheid om samen met mij het vaginaledrukinstrument met het ballonnetje te testen. En wat een komische situatie ontstond er, toen we allebei als 
vanzelfsprekend als eerste onze broek lieten zakken... Daarna vroegen we ons vertwijfeld af waar al dat water toch bleef ... Is dat normaal????:-): Deze ervaring lag ten grondslag aan het idee van een handvat van de VPI, waardoor er veel minder water nodig was en de druk meetbaar werd.

Veel dank gaat uit naar mijn promotieteam, Madelon, Marieke en Moniek, voor de begeleiding. Ik heb me zeer rijk gevoeld met jullie zorgvuldige aandacht, hulp en expertise. Jullie waren altijd bereid om nòg een keer door de stukken te gaan en van feedback te voorzien, dank voor jullie geduld om mijn grenzeloze creatieve gedachtenspinsels en eigenwijsheid te beteugelen en in goede banen te leiden.

Madelon, mijn promotor, met jouw expertise, rust, relativeringsvermogen en optimistische kijk gaf je me vertrouwen in een goede afloop. Je creërde een veilige sfeer door je bescheidenheid en humor. Marieke, wat leer ik veel van je scherpe geest en je kritische blik en het gemak waarmee jij theoretische modellen toepast. Ik kan er met grote bewondering naar kijken, èn naar het moorddadige tempo waarin je alles doet en verwoordt. Ik ben al blij als ik je kan volgen! Ik hoop dat wij in Maastricht een stevige brug kunnen bouwen tussen wetenschap en kliniek in de seksuologie. Moniek, vanaf het eerste uur tot de laatste snik stond jij aan mijn zijde. Wat heb ik eindeloos veel van je geleerd van je inhoudelijke kennis, het organiseren van een RCT-studie, je rust in het creëren van overzicht en je datafilie. Bijzonder ook dat we, zowel in als buiten ons vak veel raakvlakken hebben... bijv. uit ons dak kunnen gaan met kunstvagina's in preuts Amerika. :)

Aan de wieg van deze promotie stond mijn eerste directe-collega bij het azM, Jacques. Nog voordat dit onderzoeksproject liep hebben wij zeer prettig en nauw samengewerkt. Jij hebt me laten inzien dat de vragen die ik stelde de moeite van het exploreren waard waren. Door jou ben ik uitgenodigd om die uitdaging aan te (durven) gaan. Dank voor je begeleiding in de start van dit traject en bij de aandachtstudies.

Philip, jou wil ik graag bedanken dat je op het goede moment hebt ingegrepen en mij de weg naar de uitgang hebt gewezen. Daarmee heb je de voortgang van dit traject veilig gesteld. 
Peter, dank voor onze prettige samenwerking, het delen van jouw expertise m.b.t. de autonome appraisaltaken en je constructieve bijdrage als co-auteur aan twee artikelen. Je bevlogenheid wist je op een mooie manier te combineren met een respectvolle begeleiding. Je kunst om moeilijke zaken eenvoudig uit te leggen waren zeer geruststellend.

Veel heb ik te danken aan mijn collega's van Psychologie van het MUMC. Dymphie en Jeanette, met jullie is het team seksuologie (eindelijk) structureel uitgebreid! Het is met jullie heerlijk samenwerken en jullie bijdrage is erg welkom, verfrissend en verrijkend. Esther, Annelien, Brigitte, Gerdy, Inge, Servé, Ruud, Alied, Henriette, Bernadette, Yvonne, Chantal en alle andere collega's van psychologie: wat is het heerlijk om zulke collega's te hebben, voor het delen van lief en leed en inhoudelijke expertise. Door jullie belangstelling, support en humor krijg ik altijd weer goede zin! Sandra, wat fijn dat je nu dichter bij bent! Marjolein, heerlijk om met jou jarenlang intensief te hebben kunnen squashen. Ons onbegrensde fanatisme leidde regelmatig tot uiterst vermakelijke taferelen waar we soms van het lachen niet meer op konden staan! Veel dank gaat uit naar de dames van de poli en het secretariaat! Onze patientenzorg en onderzoek zouden nooit mogelijk zijn zonder jullie zorg, inzet en overzicht. Henny, Patricia, ik heb veel respect voor hoe jullie jarenlang het contact met onze patienten seksuologie hebben weten vorm te geven! Mijn (voormalig) leidinggevenden, Marcel en Peter† dank ik voor de gegeven ruimte en het vertrouwen met betrekking tot dit project. Rudolf, je relativisme en opportunisme waren een goede aanvulling in de begeleiding. Als ik bij je aanklop, ben je nooit te beroerd om met me mee te denken.

Al mijn opleidelingen (seksuologen, psychologen, gynaecologen en psychiaters) dank ik voor hun inzet, openheid en vertrouwen. Altijd weer geniet ik van jullie frisse blik en eigenheid waar ik zoveel van leer. Wat heb ik er mooie collega's aan over gehouden!

Mijn collega's seksuologie Selma, Daisy, Anouk, Willem-Jan, Sandrine, Sandra, Ellen, Jim, Marjo, Marieke R., Kees vd R., Theo F., de (oud)leden van de intervisiegroep en alle anderen wil ik graag bedanken voor de talloze warme en verrijkende ontmoetingen. Speciale dank gaat uit naar het Leidse team Philomene, Stephanie, Ellen, Charlotte en Aart. Bij jullie zijn is altijd een feest! Wat hebben we samen iets moois ontwikkeld! Dat smaakt naar meer! 
Christianne en Iris, mijn paranymphen, als mens en als collega uit de medische psychologie en de seksuologie lopen jullie al jarenlang met me mee en zijn julllie me allebei zeer dierbaar. We hebben veel gedeeld en veel gelachen. Ik voel me zeer dankbaar voor jullie betrokkenheid en zorg. Wat heerlijk en geruststellend dat jullie me nu en straks bijstaan!

Brechje en Iris Nowak: Naast jullie geweldige bijdragen, als KP-er-in-opleiding, aan het team seksuologie, heb ik mogen genieten van jullie als kamergenoten. Met jullie beiden heb ik veel kunnen sparren, lol gehad en lief en leed gedeeld.

De collega's van gynaecologie en urologie, met name de (oud-)collega's van VPG en van het PCcM wil ik van harte bedanken voor de jarenlange en vruchtbare samenwerking. Janneke, Arianne, Ron, Olivier, Lennie, Jacques (voor de eerste exposures!), Marga, Caroline, Mirjam, Gerold, Gerard, Jolande, Hans, Gommert, Desirée, Ernest en alle andere collegae en AIOS gynaecologie: ik heb veel van jullie geleerd en ervaar de samenwerking als buitengewoon vruchtbaar!

Dank ook aan mijn oud-collega's van de Universiteit Maastricht: Irma, voor het bieden van mijn eerste baan; Reinier, Marie-Louise, Jeanette, Geke en Susan leerden mij de kneepjes van het vaardigheidsonderwijs. Voormalig medewerkers van DMKEP bedank ik voor de prettige samenwerking waarmee we de brug vormden tussen praktijk en wetenschap. Marcel, dank voor je onnavolgzame wijsheid en kritische bevlogenheid. Angela: veel dank voor je tomeloze inzet en je gave om voor ieder probleem in no-time een oplossing te vinden.

De huidige UM FPN-medewerkers dank ik voor het kunnen sparren mbt onderzoek: Johan, voor je geruststellende wijsheid; Harald, voor je stimulerende aanmoediging; Anne, voor de VST taak; Sjaan, voor het stoere mtb-crossen.

Dit proefschrift was er nooit geweest zonder de bijdragen van een aantal mensen die als bakermat hebben gefunctioneerd voor mijn loopbaan. Fons en Sjef en het hele team vd Riagg in Echt, jullie warm, vrolijk en hecht team was voor mij de beste plek om te starten als clinicus! Medewerkers van de Riagg Maastricht, altijd weer kreeg ik bij jullie een warm welkom. Gerard, jij was een vader in de seksuologie voor mij. Ruud Bullens, jij leerde mij als clinicus academisch te werk te gaan. Het CLAS- team met o.a. Els, Arno, 
Han en Leen, geweldig om samen met jullie bevlogenheid en teamspirit het onmogelijke mogelijk te maken. Adri van Montfoort, jij begeleidde mij op kritische en humoristische wijze bij het ontrafelen van wat er in de praktijk gebeurde om de CLAS-methodiek overdraagbaar te maken.

Lieve Annette, mijn grote inspiratiebron in de partnerrelatietherapie! Veel dank voor onze gezamenlijke poli's en vruchtbare samenwerking, voor de altijd weer verrijkende ontmoetingen en warme, dierbare vriendschap.

Een goed medicijn tegen het vele zitwerk waren en zijn de Mofit sportavondjes met mijn MOFIT vriendinnen, Dianne, Brigitte, Leonne, Catharina, Bertille en Marike. Dank ook voor jullie vrolijke en stimulerende vrouwenpower! Ook mijn gezellige yogagroep onder begeleiding van Monique was en is een heerlijke verrijking.

Lieve dierbare vrienden, Hettie en Herman, Dianne en Ben, Marieke en Antoon, Ellis, Merjen, Karin en Cate, Wytske en Bard: jullie liefde, lol, betrokkenheid en zorg is een grote rijkdom in mijn leven. Ik koester alle herinneringen aan onze ontmoetingen, uitjes, logeerweekendjes en vakanties. Een basis waaruit alle uitdagingen en gedoetjes gerelativeerd en mogelijk worden. Ik hoop nog lang en vaak van jullie te genieten!

Mam en pap†, jullie hebben mij alle ruimte gegeven om te experimenteren, te ontdekken en van alles te ondernemen: Niets was te gek. Mam, jouw motto: "Spring maar, want je kunt toch zwemmen" heeft me gebracht waar ik nu sta. Pap, jouw kunst om van het leven te genieten is me altijd bij gebleven. Lieve Joos \& Fen, als jongste zus heb ik veel van jullie geleerd: Joos, van je kritische wijsheid, spiritualiteit en filosofische discussies; Fen, van je creatieve aard en praktische organisatietalent. Lieve Marja, met je gevoelige aard en vrolijke noot was jij voor mij een maatje, een warme verrijking voor ons gezin! Lieve allen, samen hebben we met alle schommelingen in het leven altijd kunnen terugvallen op onze gedeelde liefde voor sport, de natuur en dieren. Deze liefde helpt mij altijd om m'n balans te hervinden en heeft mijn leven enorm verrijkt.

Lieve schoonfamilie, mam† en pap†, Els, Jos, Caroline, Eric en Paul. Ik heb mij altijd welkom gevoeld in jullie liefdevolle warmte, zorg en vrolijke drukte. Wat fijn dat jullie mijn schoonfamilie zijn! 
Lieve Michel, jouw liefde vormt de basis in mijn leven, en dat is er na ruim 33 jaar nog iedere dag! Met jouw originele kijk, humor en autonome wijsheid weet je me altijd weer uit te dagen en wordt iedere zorg of prestatie gerelativeerd. Heel veel dank voor je uithoudingsvermogen en de ruimte die je me hebt gegeven om dit project te voltooien! We delen zoveel om samen van te genieten.

Lieve Yanick en lieve Stefan, wat is het leven mooi met jullie....en wat boffen wij met zulke "kinderen"! Het is zo leuk èn aanvullend dat jullie nu (toch :) in onze voetsporen terecht zijn gekomen! De gesprekken aan de keukentafel worden er alleen maar boeiender op! Met jullie wijsheid, reflecterend vermogen en humor houden jullie me regelmatig een kritische spiegel voor, die uiterst leerzaam en alles relativerend is. Niets is betekenisvoller dan dat jullie er zijn.

Als allerlaatste wil ik graag iedereen bij voorbaat bedanken voor alles wat uit dit proefschrift voortkomt. 

Reinhilde Melles werd geboren op 4 november 1964 te Alblasserdam. Op 10 jarige leeftijd verhuisde zij naar Zeeland, waar zij in 1983 haar VWO diploma haalde bij het CLVZ te Goes. Zij begon met de studie GezondheidsWetenschappen (GW) aan de Universiteit Maastricht (UM). In 1985 onderbrak zij deze studie om een half jaar in Frankrijk te gaan werken. Zij specialiseerde zich in de Geestelijke Gezondheidskunde (GGK), waarbij zij bij de Riagg in Echt haar praktijkstage deed, en een specialisatie seksuologie bij de Riagg Maastricht. Zij was tevens onderzoeksassistent bij de UM, actief voor het Team Seksueel Misbruik in Maastricht en voorzitter van het Samenwerkingsverband Preventie van seksueel geweld en Emancipatie van seksuele voorkeuren (SPE). In 1989 voltooide zij haar studie. Aansluitend werkte zij als docent vaardigheidsonderwijs bij de Universiteit Maastricht. Daarnaast schreef zij psychologische rapportages m.b.t. zedendelicten voor de Rechter Commissaris, Arrondissementsrechtbank te Maastricht, was docent bij de Academie Verloskunde in Kerkrade en co-therapeut bij groepen voor exhibitionisme/ voyeurisme, slachtoffers en daders van seksueel misbruik bij de NVSH te Maastricht en het Ambulant Buro Jeugdzorg te Leiden. Zij was betrokken bij het ontwikkelen van het CLAS-project (Contextuele Leergroepen voor Alle betrokkenen bij Seksueel misbruik) bij huize Clara Feij te Roermond en Jeugddorp Bethanië te Horn. T.b.v. dit project is zij in 1992 verhuisd naar Utrecht, om als projectmedewerker vanuit het Nederlands Instituut voor Zorg en Welzijn (NIZW) het CLAS-project verder te ontwikkelen, beschrijven en implementeren in andere regio's. Vervolgens werkte zij als beleidsmedewerker bij het Steunpunt Seksueel Geweld, de GGD te Amsterdam en als seksuoloog bij de Rutgerstichting. In 2000 is zij aangesteld als psycholoog-seksuoloog bij het Academisch Ziekenhuis Maastricht (azM), waar zij haar registratie als cognitief gedragstherapeut en Klinisch Psycholoog behaalde. Als reactie op bezuinigingen in de zorg, zette zij in 2003 het Regionaal Centrum Seksuologie (RCS) op, een samenwerkingsverband tussen het azM, Orbis GGZ/ Medisch Centrum, het Atrium ziekenhuis in Heerlen, de RIAGG, Propsy en de GGD. Tussen 2003 en 2013 was zij coördinator van het RCS, waarna zij teamleider seksuologie werd in het MUMC. Zij was docent bij de Universiteit Maastricht en het RINO-Zuid te Eindhoven. Hierbij ontwikkelde zij de postmaster Opleiding Seksuologie waar zij hoofdopleider van werd. 
Zij raakte betrokken bij onderzoeksprojecten, waar zij samen met het LUMC o.a. de begeleide exposure therapie voor vrouwen met vaginisme ontwikkelde en evalueerde. Dit project werd gewaardeerd met een GRANT van de European Society of Sexual Medicine. Tevens werd zij als trainer en supervisor verbonden aan Stanford Medicine University, California. Verder ontwikkelde zij een instrument om vaginale druk toe te kunnen passen in experimentele studies (Vaginal Pressure Inducer), waarvoor het recht op Europees patent is verleend door het Europees Octrooijbureau (EPO) .

De belangrijkste drijfveer van Reinhilde is het verbinden van mensen, o.a. door het onbespreekbare bespreekbaar te maken. Wat dit betreft is het bijzonder dat de betekenis van haar naam raadgeefster in de strijd is. 



\section{Articles and chapters}

Melles, R. J., Ter Kuile, M. M., Dewitte, M., Peters, M.L. (submitted) Vaginal Pressure: Pain or Pleasure? The role of individual differences and context-dependent expectations in sexual and non-sexual contexts

Dewitte, M., Schepers, J., \& Melles, R. (2018). The Effects of Partner Presence and Sexual Stimulation on the Appraisal of Vaginal Pressure and Sexual Arousal. Journal of Sexual Medicine, 15(4), 539-549

Varagnolo, D., Knorn, S., Oliver-Chiva, E., Melles, R., \& Dewitte, M. (2018). Towards the individualization of vaginal dilatation exercises: A Quantitative analysis of the variability of vaginal pressure responses. Journal of Sexual Medicine Supplement 3 15(7), S303S303

Varagnolo, D., Knorn, S., Oliver-Chiva, E., Melles, R., \& Dewitte, M. (2018). Data-driven modelling of subjective pain/pleasure assessments as responses to vaginal dilation stimuli. IEEE Control Systems Letters, 2(3), 423-428

Melles, R. J., Dewitte, M., Ter Kuile, M. M., Bonnemayer, C., Peters, M.L. The Vaginal Pressure Inducer: a new device to test the threshold of unpleasant vaginal pressure and the influence of sexual arousal. Journal of Sex and Marital Therapy, 44(2), 189-200

Varagnolo, D., Knorn, S., Melles, R., \& Dewitte, M. (2017). Qualitative modeling of pressure vs. pain relations in women suffering from dyspareuniac, IFAC PapersOnLine, 50(1), 2043-2050

Melles, R. J., Dewitte, M., Ter Kuile, M. M., Peters, M.L. \& de Jong, P. J. (2016) Attentional bias for pain and sex, and automatic appraisals of sexual penetration: Differential patterns in dyspareunia versus vaginismus? Journal of Sexual Medicine 13(8), 12551262.

Brentjens, L. B. P. M., Melles, R. J., van Hanegem, N., \& den Hartog, J. E. (2016). Psychosociale kwetsbaarheid bij dyspareunie: spelen epigenetische veranderingen daarbij een rol? Nederlands Tijdschrift voor Obstetrie \& Gynaecologie, 129, 332-337 
Ter Kuile, M. M., Melles, R. J., Tuijnman-Raasveld, C. C., de Groot, H. E., \& van Lankveld, J. J. (2015). Therapist-Aided Exposure for Women with Lifelong Vaginismus: Mediators of Treatment Outcome: A Randomized Waiting List Control Trial. Journal of Sexual Medicine, 12(8), 1807-1819

Ter Kuile, M. M., Melles, R. J., Tuijnman-Raasveld, C. C., de Groot, H. E., \& van Lankveld, J. J. (2015). Begeleide exposuretherapie voor vrouwen met primair vaginisme: een gerandomiseerde effectiviteitstudie met een wachtlijstcontrolegroep. Gezinstherapie Wereldwijd. Een selectie uit de internationale vakliteratuur, 26, 1-21

Melles, RJ, ter Kuile M.M., de Witte, M., C. Bonnemayer, Durieux, L., Peters, M.L. (2015) Invloed van seksuele opwinding bij vaginale druk: Een nieuw instrument om de beleving van inwendige vaginale druk te meten. Reproductieve geneeskunde, Gynaecologie en Obstetrie.

Melles, R. J., Ter Kuile, M. M., Dewitte, M., van Lankveld, J. J., Brauer, M., \& de Jong, P. J. (2014). Automatic and deliberate affective associations with sexual stimuli in women with lifelong vaginismus before and after therapist-aided exposure treatment. Journal of Sexual Medicine, 11(3), 786-799

Reissing, E. D., Borg, C., Spoelstra, S. K., Ter Kuile, M. M., Both, S., de Jong, P. J., van Lankveld, J. J., Melles, R. J., Weijenborg, P. T., \& Weijmar Schultz, W. C. (2014). "Throwing the baby out with the bathwater": the demise of vaginismus in favor of genito-pelvic pain/penetration disorder. Archives of Sexual Behavior, 43(7), 1209-1213

ter Kuile, M. M., Melles, R., de Groot, H. E., Tuijnman-Raasveld, C. C., \& van Lankveld, J. J. (2013). Therapist-aided exposure for women with lifelong vaginismus: a randomized waiting-list control trial of efficacy. Journal of consulting and clinical psychology, 81(6), 1127-1136

van Overveld, M., de Jong, P. J., Peters, M. L., van Lankveld, J., Melles, R., \& Ter Kuile, M. M. (2013). The Sexual Disgust Questionnaire; a Psychometric Study and a First Exploration in Patients with Sexual Dysfunctions. Journal of Sexual Medicine, 10(2), 396407 
ter Kuile, M.M. en Melles, R.J., (2010). Behandelingsstrategieën: Hoofdstuk 8 Vaginisme. In: M. Hengeveld, A. Brewaeys (red.) Behandelingsstrategieën bij seksuele disfuncties. Houten: Bohn Stafleu Loghum

ter Kuile, M. M., I. Bulte, I., P.T. Weijenborg, A. Beekman, R. Melles, P. Onghena (2009) Therapist-aided exposure for women with lifelong vaginismus: a replicated single-case design Journal of Consulting and Clinical Psychology 77 (1) 149-159

ter Kuile, M. M., van Lankveld, J. J., Groot, E. D., Melles, R., Neffs, J., \& Zandbergen, M. (2007). Cognitive-behavioral therapy for women with lifelong vaginismus: Process and prognostic factors. Behaviour Research and Therapy 45(2), 359-373

Melles, RJ; E. de Groot, J. van Lankveld, M. ter Kuile, J. Nefs, M. Zandbergen (2006) De effectiviteit van cognitieve gedragstherapie middels groeps- en bibliotherapie bij primair vaginisme: De resultaten van een gerandomiseerde gecontroleerde studie. Tijdschrift voor Seksuologie, 30, 195-203

van Lankveld, J. J., ter Kuile, M. M., de Groot, H. E., Melles, R., Nefs, J., \& Zandbergen, M. (2006). Cognitive-behavioral therapy for women with lifelong vaginismus: a randomized waiting-list controlled trial of efficacy. Journal of Consulting and Clinical Psychology, $74(1), 168-178$

E. de Groot, R. Melles, M. ter Kuile en J. van Lankveld, Primair vaginisme, groepsprotocol, LUMC \& AZM ,2002

E. de Groot, R. Melles, M. ter Kuile en J. van Lankveld, Dyspareunie, groepsprotocol, LUMC \& AZM, 2002

Melles, R. (2002) Verslag studiedag: Geen zin in seks, Tijdschrift voor seksuologie, 26 (2)

Melles, R. (2001) Libido kun je leren, De psycholoog, 36, (12)

J.van Lankveld, M. ter Kuile, R. Melles en E. de Groot, Zelf je vaginistische reactie overwinnen, azM, LUMC

Van der Rhee, C. en R. Melles (1999), Terugvalpreventieproject jeugdige zedendelinquenten Rutgers Stichting, Rutgers Consult, Utrecht 
Bögels, S.M. Vleuten C.P.M., G. Blok, Kreutzkamp, R., Melles R., Schmidt, H.G. (1995) Assessment and validation of diagnostic interviewing skills for the mental health professions Journal of Psychopathology and Behavioral Assessment 08, 17(3):217-230

Melles, R.J., N Bakhuizen en A.J. van Montfoort (1996), Contextuele hulpverlening na seksueel misbruik. Ontwikkeling en werkwijze van het CLAS-project, VU uitgeverij, Amsterdam

Bruggeman-Kluvers, M., R. Melles en A.J. van Montfoort (1996), Hulpverlening en casemanagement. Naar een samenhangende aanpak van seksueel misbruik van kinderen door verwanten, $V U$ uitgeverij, Amsterdam

Melles, R.J. (1994), Meersporenbeleid is aan vernieuwing toe. Onderzoek naar de aanpak van seksueel misbruik binnen het gezin. Tijdschrift voor Jeugdhulpverlening en Jeugdwerk,6, 1994

Melles, R.J. en A.J. van Montfoort (1993), Het meersporenbeleid in de praktijk gevarieerd, NIZW, Utrecht

Melles, R.J. (1991), Projectplan CLAS, Contextuele Leergroepen voor Alle betrokkenen bij Seksueel misbruik, Jeugddorp Bethanië, Horn

\section{Recent International conference presentations}

Melles, R.J. (2018) Emotionally Focused Therapy in Couples with Sexual Problems. Paper presented at the 14th Congress of the European Federation of Sexology. Albufeira, Portugal

Melles, R.J., ter Kuile,M.M., (2017) GET-PEN course, 2-weeks training in Therapist-aided Exposure Treatment, Department of Psychiatry and Behavioral Sciences, Stanford Medical Center, Stanford, California

Melles, R.J., ter Kuile,M.M., de Groot, E., Tuijnman, C., Weijenborg, P. (2017) TherapistAided Exposure Treatment. Presentation, demonstration and training on behalf of 
collegaes of the Department of Psychiatry and Behavioral Sciences, Stanford Medicine, LUMC, Leiden

Melles, R. J., Dewitte, M., Ter Kuile, M. M., Bonnemayer, C., Peters, M.L. Vaginal pressure: pain or pleasure? Influence of sexual arousal. Poster presented at the CHAMP-conference, the Center for Health and Medical Psychology (CHAMP) at Örebro University, Sweden

Melles, R.J., (2016) Vaginal pressure: pain or pleasure? Influence of sexual arousal. Presentation on the $18^{\text {th }}$ Congress of the International Society of Psychosomatic Obstetrics and Gynaecology (ISPOG), Málaga, Spain

Melles, R.J., (2016) Fear and pleasure of penetration. The effect of exposure in women with vaginismus and sexual abuse experiences. Presentation for the 41st European Association for Behavioural and Cognitive Therapies Annual Congress, Stockholm, Sweden

Melles, R.J., ter Kuile,M.M., (2016) Women with lifelong vaginismus. 'State of the art'. The Women's Health and Wellness Advanced Clinical Didactic Workshop: Trauma and Genito-Pelvic Pain/Penetration Disorders. Assessment and Treatment of Genito-Pelvic Pain/Penetration Disorder in Women with Interpersonal Violence Exposure For the Department of Psychiatry and Behavioral Sciences, Stanford Medicine

Melles, R.J., (2011). Sex and threat in women with vaginismus before and after exposure treatment. Presentation for the 41st European Association for Behavioural and Cognitive Therapies Annual Congress, Reykjavik, Iceland

Melles, R.J., (2011) Sex and threat in women with vaginismus before and after exposure treatment. Presentation for an international meeting, Pelvic Care Centrum, Maastricht

\section{Recent National conference presentations}

Melles, R.J., Laan, E. (2018) Kom op CBT-er: En nu ook de seks!! Clinical Master Class gegeven op het najaarscongres Cognitieve Gedragstherapie, Veldhoven 
Melles, R.J., In de Braek, D. (2016) Diagnostiek en behandeling van seksuele problematiek als gevolg van hersenletsel. Workshop gegeven op het Neuropsychotherapiecongres, Driebergen

Melles, R.J., (2016) Vaginale druk: pijn of fijn? Invloed van seksuele opwinding. Presentatie gegeven op symposium vaginale penetratie op het najaarscongres Cognitieve Gedragstherapie, Veldhoven

Melles, R.J., (2015) Vaginale druk bij dyspareunie: ontwikkeling van een instrument om pijn appraisal, pijntolerantie en coping met pijn te meten.Presentatie gegeven op het $20 \mathrm{e}$ Nederlands-Vlaams Doelencongres Infertiliteit,Gynaecologie en Obstetrie, de Doelen, Rotterdam

Melles, R.J., (2014). Vaginale druk: Invloed van seksuele opwinding, Presentatie gegeven op de wetenschapsdag van de Nederlandse Vereniging voor Seksuologie, Amsterdam

Melles, R.J., (2014) Pijnlijke seks: Vermijden of persisteren? Presentatie gegeven op de Refereerbijeenkomst seksuologie: Seks in Limburg. Onderzoek van eigen bodem, Heerlen

Melles, R.J., Cuypers, W.J. (2010-2014) Praten over seks in de psychiatrie. Diverse presentatie en workshops gegeven t.b.v. nascholing psychiatrie

Melles, R.J., Cuypers, W.J. (2013) Train-de-trainers workshop gegeven voor Psychiaters en Seksuologen, Utrecht

Melles, R., ter Kuile,M.M., de Groot, E., Tuijnman, C. van der Waart, T., Weijenborg, P. Beekman, A. (2009) Begeleide exposure bij vrouwen met primair vaginisme. Tot of over de grens? Presentatie gegeven op de Algemene Ledenvergadering van de Nederlandse Vereniging voor Seksuologie, Utrecht

Melles, R., (2008) Het hanteren van angst en vermijding in seksuele therapie. Mislukkingen, valkuilen en tips in de behandeling van primair vaginisme. Presentatie gegeven op de GGZ Oost-Brabant, Boekel 
Melles, R., ter Kuile,M.M., de Groot, E., (2006) Primair vaginisme: een eenvoudig en succesvol te behandelen klacht? Workshop gegeven op het congres Cognitieve Gedragstherapie, Veldhoven

Melles, R., ter Kuile,M.M., de Groot, E., van Lankveld J.L., (2006). Vaginisme. Klacht \& patiënt kenmerken. Presentatie gegeven op de Riagg, Maastricht

\section{PATENT}

2014

Inventor of Vaginal Pressure Inducer, European Patent Office

\section{GRANT}

2007 ESSM Grant for Medical Research 2007. "Prolonged therapist-aided exposure treatment for women with lifelong vaginismus: A randomized waiting-list control trial of efficacy" 


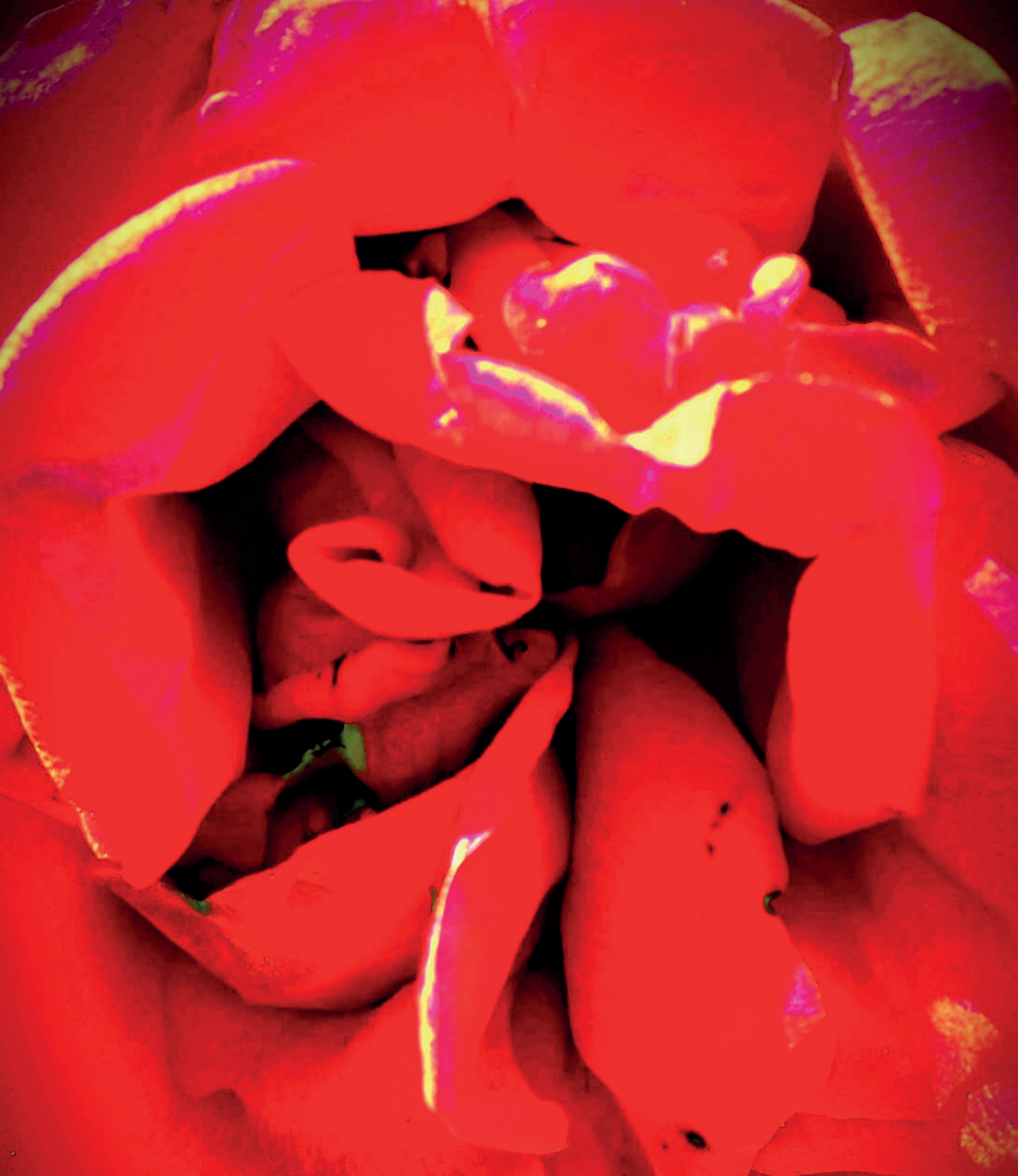

